\title{
Spatial attention in neutral and negative affect : behavior and electrophysiology
}

Citation for published version (APA):

Jongen, E. M. M. (2007). Spatial attention in neutral and negative affect : behavior and electrophysiology. [Doctoral Thesis, Maastricht University]. Datawyse / Universitaire Pers Maastricht. https://doi.org/10.26481/dis.20070419ej

Document status and date:

Published: 01/01/2007

DOI:

10.26481/dis.20070419ej

Document Version:

Publisher's PDF, also known as Version of record

\section{Please check the document version of this publication:}

- A submitted manuscript is the version of the article upon submission and before peer-review. There can be important differences between the submitted version and the official published version of record.

People interested in the research are advised to contact the author for the final version of the publication, or visit the DOI to the publisher's website.

- The final author version and the galley proof are versions of the publication after peer review.

- The final published version features the final layout of the paper including the volume, issue and page numbers.

Link to publication

\footnotetext{
General rights rights.

- You may freely distribute the URL identifying the publication in the public portal. please follow below link for the End User Agreement:

www.umlib.nl/taverne-license

Take down policy

If you believe that this document breaches copyright please contact us at:

repository@maastrichtuniversity.nl

providing details and we will investigate your claim.
}

Copyright and moral rights for the publications made accessible in the public portal are retained by the authors and/or other copyright owners and it is a condition of accessing publications that users recognise and abide by the legal requirements associated with these

- Users may download and print one copy of any publication from the public portal for the purpose of private study or research.

- You may not further distribute the material or use it for any profit-making activity or commercial gain

If the publication is distributed under the terms of Article $25 \mathrm{fa}$ of the Dutch Copyright Act, indicated by the "Taverne" license above, 


\section{Spatial Attention in \\ Neutral and Negative Affect}

Behavior and Electrophysiology

Ellen M.M.Jongen 


\section{Spatial Attention in Neutral and Negative Affect

\author{
behavior and electrophysiology
}


Cover design by Hub Ermers

ISBN 978-90-5278-622-3

Universitaire Pers Maastricht

Copyright (C Ellen M.M. Jongen, Maastricht 2007 


\title{
Spatial Attention in Neutral and Negative Affect
}

\author{
behavior and electrophysiology
}

\section{Proefschrift}

ter verkriiging van de graad van doctor aan de Universiteit Maastricht, op gezag van de Rector Magnificus, prof. mr. G.P.M.F. Mols, volgens het besluit van het College van Decanen, in het openbaar te verdedigen op donderdag 19 april 2007 om 14.00

door

Ellen Mathea Maria Jongen 


\section{Promotor}

Prof. dr. H.L.G.J. Merckelbach

\section{Copromotor}

Dr. F.T.Y. Smulders

\section{Beoordelingscommissie}

Prof. dr. A. Arntz (voorzitter)

Prof. dr. R. Goebel

Dr. L.M. Jonkman

Prof. J.L. Kenemans (Universiteit Utrecht)

Dr. C. Nederkoorn

The research presented in this dissertation was funded by the Netherlands Organization for Scientific Research (NWO, The Hague, grant number 425-20-308) awarded to Dr. F.T.Y Smulders and Prof. Dr. H.L.G.J. Merckelbach. 


\section{Contents}

1. General Introduction 7

2. Varieties of attention in neutral trials: Linking RT to ERPs and EEG frequencies 33

3. Lateralized ERP components related to attentional orienting in an all-go and a go-nogo task: Discriminating shifts of attention from sensory aspects of the cue 53

Experiment 1: 56

Experiment 2: 69

4. Sequence effects in a spatial cueing task: Endogenous orienting is sensitive to orienting in the preceding trial 83

5. Attentional bias and general orienting processes in bipolar disorder 95

6. The time course of general orienting and attentional bias for emotional stimuli in induced and naturally-occurring dysphoria: Response Times, Eye Movements, and EEG 109

7. General Discussion 151

8. Summary 167

9. Samenvatting 173

References 181

Dankwoord 199

Curriculum Vitae 203 



\section{General Introduction}


Take a minute to think about how you would define "attention". Most people are able to come up with a description. However, these descriptions probably are very diverse, concerning many different situations. This demonstrates the diversity of mechanisms that are all indicated by the term attention. An exact operational definition therefore is necessary before one can start studying attention.

The central theme of this thesis is selective attention. Selective attention is one of our crucial cognitive mechanisms that functions as a guide in everyday life, helping us to prioritize (or select) relevant information and at the same time inhibit less important information. This is necessary because our cognitive resources are limited and in most everyday situations we are bombarded with all kinds of information. Selective attention can concern different modalities. In this thesis, the discussion will be limited to the visual domain. One of the main goals of this thesis was to derive sensitive indices of orienting in the visual domain and use these to measure attention with a high temporal resolution. The relation between attention and negative mood was examined using these indices of orienting to study some pertinent issues regarding the claimed bias of attention in depression. Finally, it was examined how attention can be influenced by preceding events.

The goal of this chapter is to introduce the themes and the research methods that are central to this thesis. The two main themes are visual spatial attention and the interplay of attentional bias and negative mood. The measures that were used in this thesis are response times, measures derived from the EEG, and eye movements. The chapter ends with a general lay-out of the thesis.

\section{Attention}

Posner and Boies (1971; Posner \& Petersen, 1990) discriminated processes of attention that heighten general alertness and processes that selectively prepare a subject, for example for a location. The increase in general alertness has been addressed by experiments that used warning signals (e.g. Bertelson, 1967; Woodrow, 1914). These experiments showed that performance is improved when a warning signal is given and target stimuli appear at a predictable interval. Selective preparation for a location, indicated as "spatial selective attention", is usually studied with a spatial cueing paradigm (e.g., Posner, Nissen, \& Ogden, 1978: Posner, Snyder, \& Davidson, 1980). Adjusted versions of this paradigm were used in each of the empirical chapters in this thesis. In most spatial cueing paradigms, a cue indicates the likely location of an upcoming target stimulus. The goal of most cueing studies is to assess covert shifts of attention. These are defined as shifts of attention without co-occurring movements of the eyes (e.g., Posner, 1980). When the eyes are moved and a target position is fixated, faster and more accurate performance would be expected simply because of the greater density of photoreceptors in the fovea. Therefore, to study the effects of attention, subjects are usually instructed to maintain central fixation. Furthermore, subjects are instructed to respond to every target as fast as possible, usually by pressing a button. On most of the trials, the target is presented at the position that was cued, and these are called "valid trials". In some trials the target appears at the uncued location, and these are called "invalid trials". In many experiments, neutral trials are also included, in which both locations are cued. Typically, reaction times (RTs) are faster and accuracy is higher for 
validly cued targets than for invalidly cued targets. This RT difference is called "the validity effect". Performance on neutral trials usually is in between. It is assumed that after a neutral cue attention is not oriented but kept at fixation until target-onset (Posner, 1978). Some alternatives regarding attentional processing following a neutral cue have been proposed by De Gonzaga and colleagues (De Gonzaga, Gawryszewski, Riggio, Rizzolatti, \& Umiltá, 1987). First, attention may switch between the target positions. Second, instead of a focused state of attention, neutral trials might be characterized by the diffuse state of attention that was postulated by Jonides (1983). Third, attention might be disengaged and ready to move after a neutral cue is presented. Following the assumption that attention remains at fixation, the RT-difference between neutral trials and invalid trials reflects the cost of orienting attention to the wrong location, and the RT-difference of neutral trials and valid trials reflects the benefit of orienting attention to the correct location. ${ }^{1}$ Orienting can be divided in three mental sub-operations: disengagement from the current focus of attention, a shift of attention to a target location, and engagement at this location (Posner, Walker, Friedrich, \& Rafal, 1984). Costs and benefits of attention have been described in terms of these mental operations (Posner et al., 1984; for an illustrative example, see Clark, Geffen, \& Geffen, 1989). That is, when it is assumed that attention is engaged at fixation in neutral trial, benefits represent the temporal advantage that results from movement and engagement of attention at the cued location before target presentation, and costs represent disengagement from the invalid location, movement to, and re-engagement at the correct location following target presentation.

Two kinds of cues can be discriminated and each is related to another type of attention (Posner, 1980). First, symbolic cues, for example arrow cues, can be presented at the centre and predict the target position with a probability above chance. Participants can orient their attention to the indicated position and this type of orienting, termed endogenous orienting, is thought to be controlled and voluntary (Jonides, 1981). Alternatively, cues can be presented at one of the target positions, for example by presenting an increase in luminance. These cues will attract attention even when they do not predict the target position, and are therefore thought to capture attention automatically and in that sense involuntarily (Jonides, 1981; Rafal \& Henik, 1994). This type of attention has been termed exogenous orienting. Although both types of cues lead to improvement in performance, the time course of endogenous and exogenous orienting differs. First, exogenous cues lead to faster shifts of attention than endogenous cues (Müller \& Rabbitt, 1989). Second, whereas facilitation of target processing at endogenously cued positions remains present until after a second (after cue-onset), the facilitation effect for exogenous cues reverses after about $300 \mathrm{~ms}$ : responses for targets that appear at the indicated location become slower and errors increase relative to targets at the uncued location (Posner \& Cohen, 1984). This effect was called

1 Nobre and colleagues have shown that similar to attention in space, people are able to orient attention in time (e.g., Coull \& Nobre, 1998). This has been demonstrated by using an analogue of the Posner cueing paradigm with temporal cues that indicated if a target would appear after a short or long interval with $80 \%$ predictive validity. Behavioral validity effects were found and this effect of "temporal orienting" has abundantly been replicated (for a review, see Nobre, 2001). 
“inhibition of return” (IOR; Posner, Rafal, Chaote, \& Vaughan, 1985). It has been suggested that IOR might function to encourage orienting to new locations, or similarly to discourage re-inspections of old locations (e.g., Cheal \& Chastain, 1999; Clohessy, Posner, Rothbart, \& Vecera, 1991; Klein, 1988; Posner \& Cohen, 1984; Rafal \& Henik, 1994). These explanations have recently been questioned (Berlucchi, 2006). IOR has not been demonstrated with endogenous orienting unless saccade preparation is allowed (Rafal, Calabresi, Brennan, \& Sciolto, 1989). For a full description of IOR, the interested reader is referred to excellent reviews (Klein, 2000; Lupiáñez, Klein, \& Bartholomeo, 2006; Taylor \& Klein, 1998).

An important issue in attention literature is the locus-of-selection problem which concerns the stage of information processing that is affected by attention. Whereas Posner and colleagues proposed that RT speeding on valid trials was an effect of enhanced perceptual processing (Posner, 1980; Posner, Nissen \& Ogden, 1978), others have argued that it might be the result of changes in decision bias, thus representing later processing stages (Shaw, 1984; Sperling, 1984). It is difficult to isolate the locus of selection on the basis of behavioural response data. However, electrophysiological measures have delivered some important insights in the discussion of early or late selection, as discussed below.

\section{Measures derived from the EEG}

The electroencephalogram (EEG) is a recording of electrical brain activity. One of the strong points of EEG is its perfect temporal resolution. This allows mapping the time course of cognitive processes with great precision. In the EEG experiments described in the present thesis two measures were derived from the EEG: (1) Event-Related Potentials (ERPs), and (2) Event-Related changes in neural oscillations. The fact that both measures are "event-related" indicates that they are related in time to an internal or external event. This is what makes them so interesting for psychologists. A short description of both is given.

ERPs and event-related changes in oscillations are both constituents of the online EEG. To highlight an ERP, repetitions of the same event (and hence responses to this event) are simply cut out of the EEG (or "epoched") and averaged. Background EEG activity that is non-phase-locked is attenuated this way. However, this non-phase-locked background activity that many ERP researchers think of as noise also includes eventrelated activity of interest. Non-phase-locked activity is used to describe changes in oscillations that are specific for different frequency bands. To extract the frequency bands of interest, a filtering operation is necessary. Various frequency bands have been related to cognitive mechanisms. Below, research that has examined frequency bands related to attention will be described. After filtering, but before the averaging operation, activity is either squared, or the absolute values of the amplitudes are calculated. This choice describes the most important difference between the classical Event-Related Desynchronization / Synchronization (ERD/ERS) method (Pfurtscheller \& Aranibar, 1977) that represents changes in power and therefore is based on the squared average, and Temporal Spectral Evolution (TSE, Salmelin \& Hari, 1994) that represents changes in amplitude. One of the advantages of taking the absolute values is that the resulting measure is expressed in the same unit of measurement as ERPs. As noted by 
Kalcher and Pfurtscheller (1995), changes in oscillations that are calculated by bandpass filtering, squaring, and averaging still contain phase-locked activity. In order to discriminate phase-locked from non-phase-locked event-related EEG activity the intertrial variance method was proposed which boils down to subtracting the filtered ERP from every single trial band-pass filtered epoch before squaring and averaging the data. These changes in oscillations, free from phase-locked activity, are indicated as induced changes in oscillations (Kalcher \& Pfurtscheller, 1995).

The ERP can be described in terms of positive and negative peaks, which are called components. Components are believed to reflect synchronized postsynaptic activity in large populations of neurons. Components are related to sensory, motor, or cognitive processes of interest and whereas the latency of these components is used to indicate the time course of these processes, their location can be used to estimate the neural generators. However, spatial resolution of EEG is relatively poor. Event-related changes in oscillations are described in terms of decreases or increases of neural activity with respect to a pre-stimulus baseline. Whereas negative amplitude values represent a decrease of activity that is related to desynchronization of neural firing, positive amplitudes represent an increase of activity that is related to synchronization of neural firing. Importantly, these changes in oscillations are always defined within a frequency band of interest as oscillations in specific frequency bands are thought to have distinct functional roles. These descriptions illustrate an interesting difference between ERPs and event-related changes in oscillations: negative and positive amplitude (or power) values only have got differential functional meaning when studying changes in oscillations.

\section{Eye movements and attention}

There is no doubt that covert selection and overt selection are closely related. However, their relation appears to be asymmetric as attention can be shifted without moving the eyes, but planning and carrying out an eye movement appears to require a shift of attention to the goal position of eye movements first (for a review, see Kowler, in press). A longstanding issue concerns the underlying mechanism of these processes (for a review, see Awh, Armstrong, \& Moore, 2006). According to the premotor theory of attention, eye movements and covert shifts of attention reflect essentially the same process, originating in the same neural areas (e.g., Rizzolatti, Riggio, Dascola, \& Umilta, 1987), and directing attention to a location is thought to be equivalent to preparation of the eyes to move to this location. However, others have argued that covert selection and overt selection rely on independent mechanisms (e.g., Juan et al., 2004; Klein, 1980). This discussion appears difficult to solve (Awh et al., 2006). Still, in those conditions where attention and eye movements are linked, attention appears to be the mechanism that guides the movement of the eyes (e.g., Deubel \& Schneider, 1996; Kowler, Anderson, Dosher, \& Blaser, 1995; McPeek \& Keller, 2002; McPeek, Maljkovic, \& Nakayama, 1999; Posner, 1980; Posner \& Cohen, 1984).

\section{ERP studies of attention}

In an ERP study of anticipatory attention, a warning stimulus was presented, followed one second later by a response stimulus (Walter, Cooper, Aldridge, McCallum, \& Winter, 1964). In the interval between the two stimuli, a negative slow potential was recorded 
that was called the contingent negative variation (CNV, or "expectancy wave"). Across trials, an increase in the amplitude of this component was related to a reduction of RT (see also Hackley and Miller, 1995). In the paradigm used by Walter et al., after the warning stimulus two processes were triggered: preparation for movement (to respond) and anticipatory attention for the stimulus. The CNV therefore is considered to reflect both processes. Damen and Brunia (1987; Brunia \& Damen, 1988) were able to discriminate these two processes, each characterized by a negative wave (for reviews, see Brunia, \& van Boxtel, 2001; van Boxtel \& Böcker, 2004).

In addition to these studies on general alertness, ERP studies on spatial selective attention in the visual domain have been conducted. In many of these, the modulating effects of attention on target porcessing have been studied. When a target stimulus is presented, a sequence of ERP components is evoked over lateral occipital sites consisting of an early P1 component (onset at about 70-90 ms), followed by an $\mathrm{N} 1$ component (onset at about 100-120 ms) (for reviews, see Anllo-Vento, Schoenfeld, \& Hillyard, 2004; Luck, Woodman, \& Vogel, 2000). For validly cued stimuli in comparison to invalidly cued stimuli, amplitude enhancements for both components are typically found (e.g. Eason et al., 1969; Mangun, Hansen, \& Hillyard, 1986; Mangun, Hillyard, \& Luck, 1993). This finding has been important for the debate on the stage of information processing affected by attention. It shows that attention can affect sensory processing and therefore provides evidence in favor of early selection models of attention.

In an increasing number of spatial cueing studies, the process of orienting is now also studied after cue-onset. These studies have focused on so-called lateralized components, with a positive or negative voltage over the hemisphere contralateral to the direction of attention. Lateralized components are of interest, because the left and the right hemisphere both carry out the operations necessary for shifts of attention in the contralateral direction. In Table 1.1, an overview is given of studies that used visual cues to direct attention and examined these lateralized components. Note that there also is an expanding literature of studies on ERPs after cue-onset that examined cross-modal attention and for example used auditory or tactile cues (e.g. Eimer \& van Velzen, 2002; Eimer, van Velzen, \& Driver, 2002; Green \& McDonald, 2006). Harter and colleagues (Harter, Miller, Price, LaLonde \& Keyes, 1989) were the first to describe ERP components in the cue-target interval, and a sequence of lateralized components has since repeatedly been reported (see Table 1.1). This sequence consists of an early directing attention negativity (EDAN) at posterior electrodes between about 200 and $400 \mathrm{~ms}$ post cue, an anterior directing attention negativity (ADAN) at frontal and central sites between 300 and $500 \mathrm{~ms}$ post cue, and a late directing attention positivity (LDAP) at posterior sites that starts around $500 \mathrm{~ms}$ post cue. These components are suggested to reflect different stages in the control of attention. The EDAN has been hypothesized to reflect encoding of the meaning of a cue, and the resulting initiation of an attentional shift to the cued location (Harter et al., 1989; Hopf \& Mangun, 2000). However, Van Velzen and Eimer (2003) suggested that the component indicated by the EDAN was similar to another lateralized negativity; the N2pc component (discussed below). In their studies, a special type of cue was used that consisted of a relevant and an irrelevant part placed left and right from fixation. One of the parts pointed to the left and the other part pointed to the right. It was shown that the EDAN was negative in amplitude contralateral to the position of the relevant part, irrespective of the cued 
location. Therefore, it was concluded that the EDAN was an N2pc component, reflecting the filtering of irrelevant or the selection of relevant information (Luck \& Hillyard, 1994; Eimer, 1996). However, as the cues used in their experiment required selection of a lateralized part, one might wonder if these conditions, ideal to evoke an N2pc, are actually comparable to those when standard arrow cues are used. The LDAP has been assumed to reflect modulation of the cortical excitability in those regions that process the upcoming visual information (Harter et al., 1989). However, inconsistent with this interpretation, the LDAP decreased before target onset (Hopf \& Mangun, 2000), and when SOA was varied it was shown to be time-locked to cue onset instead of target onset (Van Velzen, Forster, \& Eimer, 2002). Furthermore, as shown in Table 1.1, no LDAP was found in a number of cueing studies which suggests that it is not required for orienting. In line with the common view that frontal cortical structures contribute to the attentional control of sensory processing (e.g., LaBerge, 1997; Posner \& Petersen, 1990), the ADAN has been associated with attentional control and redirecting attention in space.

Selection of lateralized stimuli elicits a negative component over contralateral posterior sites, around 200 ms after onset of these stimuli. This so-called N2pc (an abbreviation of N2-posterior-contralateral) component was demonstrated for the first time in a visual search task, and was interpreted as a reflection of spatially filtering irrelevant information (Luck \& Hillyard, 1994, Woodman \& Luck, 1999; Woodman \& Luck, 2003). As it was also elicited when spatial filtering was less important, in conditions where only two stimuli were presented in opposite hemifields (Eimer, 1996; and see also Robitaille \& Jolicoeur, 2006; Wijers, Lange, Mulder, \& Mulder, 1997), Eimer suggested that it might be a reflection of detection and selection processes that are independent of the number of distracting items. However, Luck and colleagues showed that the amplitude of the N2pc was sensitive to the need to focus attention as it was larger when more items were presented relative to one in each hemifield, and in a complex conjunction task as compared to a simple detection task (Luck, Girelli, McDermott, \& Ford, 1997). Finally, the component was elicited when selection was based on physical characteristics (Eimer, 1996, experiment 1 and 2; Luck \& Hillyard, 1994; Robitaille \& Jolicoeur, 2006), and when selection was based on word meaning (Eimer, 1996, experiment 3).

In sum, a number of lateralized components are related to the process of orienting and selection processes of attention. More research appears necessary on the exact functional significance of these lateralized ERP components.

\section{Oscillations and attention}

It has repeatedly been shown that decreases in the alpha band represent a correlate of activated cortical areas (e.g. Klimesch, 1999; Klimesch et al., 1996), and increases correspond to decreased functional activity in the underlying cortex (e.g. Pfurtscheller, 1992; Pfurtscheller, Stancák, \& Neuper, 1996). The latter was supported by an fMRI study (Goldman, Stern, Engel, \& Cohen, 2002) where increases of alpha were correlated with decreased activity in multiple regions of the cortex. Decreases in the alpha band have repeatedly been related to increases in attention and activation (e.g. Boiten, Sergeant, \& Geuze, 1992; Dujardin et al., 1993; Vazquez Marrufo, Vaquero, Cardoso, \& Gómez, 
2001) and stimulus anticipation (Bastiaansen \& Brunia, 2001; Filipovic, Jahanshahi, \& Rothwell, 2001; Bastiaansen, Böcker, \& Brunia, 2002).

Selective spatial attention has been related to changes in oscillations in the alpha band and the gamma band. In an endogenous spatial cueing paradigm, lateralized changes in the alpha band were measured after cue-onset (Worden, Foxe, Wang, \& Simpson, 2000). There was an increase in the alpha band at occipital sites, ipsilateral to the cued location and therefore contralateral to the position that was to be ignored. Comparable increases of alpha have also been shown in cross-modal cueing paradigms, where parieto-occipital alpha increased when the visual modality had to be ignored (Foxe, Simpson, \& Ahlfors, 1998; Fu et al., 2001). These increases of alpha have been interpreted as active gating of distractor stimuli and uncued spatial locations. In addition to alpha activity, gamma activity has been shown to be related to attentional cueing. It has been shown that gamma activity increases with high arousal, alertness and attention (Bouyer, Montarom, Vahnee, Albert, \& Rougeul, 1987; De Pascalis \& Ray, 1998; Müller, Gruber, \& Keil, 2000; Sheer, 1976; Shibata et al., 1999; Sokolov et al., 1999) and is related both to bottum-up and top-down attentional processing (for reviews see Müller and Gruber, 2001; Keil, Gruber, \& Müller, 2001). In an endogenous spatial cueing paradigm, gamma activity $(35-51 \mathrm{~Hz}$ ) was measured after cue-onset (Gruber, Müller, Keil, \& Elbert, 1999). A broad posterior distribution before cue-onset changed to an increase of power at parieto-occipital sites, contralateral to the to-be-attended side.

In sum, in a small number of studies on attentional orienting, the direction of attention was shown to be related to contralateral decreases in the alpha band or contralateral increases in the gamma band.

\section{Mood disorders}

Mood disorders according to DSM-IV (American Psychiatric Association, 1994) are divided into Depressive Disorders (unipolar depression) and Bipolar disorders. Whereas manic, hypomanic, and mixed episodes discriminate bipolar disorders from unipolar depression, major depressive episodes are characteristic for both types of disorders. Major depressive episodes, do not refer to the symptom of "feeling down" but rather represent a syndrome that consists of a number of physical and mental symptoms. Core symptoms of a depressive episode according to DSM-IV are a depressed mood and loss of interest or pleasure. In addition to these, at least three other of the following should be present: weight or appetite gain or loss, insomnia or hypersomnia, psychomotor agitation or retardation, fatigue or loss of energy, sense of worthlessness or excessive guilt, reduced concentration or indecisiveness, and recurrent thought of death or suicidal ideation. The symptoms must cause distress and impairment in social, occupational, or other areas of daily functioning.

An often used questionnaire, sensitive in measuring depression, is the Beck Depression Inventory (Beck, Ward, Mendelson, Mock, \& Erbaugh, 1961). The BDI, although meant to measure the syndrome depression and not just negative affect, is not intended to be a screening device (Kendall, Hollon, Beck, Hammen, \& Ingram, 1987). Therefore, unless diagnostic procedures have shown that BDI-evaluated samples meet criteria for clinical depression, subjects scoring high on the BDI are preferably indicated by the term "dysphoric" instead of depressed. Correlations between the BDI and similar 
kinds of questionnaires (e.g. the Hamilton Rating scale for Depression) are high (Beck, Steer, \& Garbin, 1988). Therefore, other questionnaires can serve the same purpose of discriminating groups of dysphorics. The present thesis will focus on dysphoria and bipolar depression.

\section{Attentional bias in unipolar depression and dysphoria}

According to cognitive models of emotional processing (e.g. Beck, 1976; Bower, 1981) depression is characterized by a selective processing bias for mood-congruent information in all cognitive processes. The bias is expected to be specific for information that is linked to one's current life concerns (Beck, 1987). Empirical support for these predictions is far from conclusive although there is strong evidence for biased retrieval processes in depression (Williams, Watts, Macleod, \& Mathews, 1997). The work presented in this thesis will focus on attentional bias in dysphoria and bipolar depression. According to Williams et al. (1997) an attentional bias occurs "when there is a discrete change in the direction in which a person's attention is focused so that he/she becomes aware of a particular part or aspect of his/her stimulus environment" (p.73). More generally stated, when attention is biased, certain stimuli or aspects of a stimulus are processed in a preferential way relative to other stimuli or aspects of a stimulus. Several paradigms drawn from cognitive psychology have been adjusted to study attentional bias for emotional stimuli. These are described in Box 1.

Studies of attentional bias can be divided into studies on interference, most frequently measured with a Stroop task, and studies on selective attention, most frequently measured with a dot-probe task, but also by use of an attentional deployment task, or a visual search task. It is unclear whether interference on the Stroop task is a reflection of competition at the input or the output of information processing (Macleod, 1991; De Ruiter \& Brosschot, 1994). Biases on a Stroop task may therefore either reflect processes of attention or processes of response selection. The discussion here will be limited to measures of selective attention. Therefore, hereafter when the term "attentional bias" is used, it refers to measures of selective attention(al bias). For an overview of results on other types of attentional bias and cognitive biases in depression and dysphoria the interested reader is referred to recent reviews (Clark \& Beck, 1999; Mathews \& Macleod, 2005; Mogg \& Bradley, 2005).

In Table 1.2, presented at the end of this chapter, all attentional bias studies with depressed and dysphoric participants in which measures of selective attention were used are summarized. In some of these, the main focus was on anxiety, but measures of depression were used in additional analyses. For all studies only results regarding depression and dysphoria are mentioned. The dot probe task was developed by Macleod, Mathews, and Tata (1986). They found no evidence for an attentional bias in a group of participants with major depression (MD). Hill and Duiton (1989) used the same task with self-esteem threatening words and also found no evidence for an attentional bias in dysphoria. Although the original dot probe task by Macleod et al. (1986) was innovative, it had two important drawbacks that complicate the interpretation of the results. First, probe stimuli were presented more often after threat stimuli than after neutral stimuli. Consequently, threat stimuli had a predictive value concerning the probe. Second, participants were asked to read aloud the top word on every trial. Attention thus 


\section{BOX 1 \\ Paradigms to study attentional bias}

Emotional Stroop task (for reviews, see Melara \& Algom, 2003): this task is adapted from a regular Stroop task (Stroop, 1935) and is the paradigm that has most often been used to study attentional bias. Participants are shown words of emotional or neutral content in different colors and are required to name the color of the words as fast as possible while ignoring word meaning. The effect of interest is preferential processing of mood-congruent word stimuli indicated by response slowing.

Emotional Visual search task (e.g., Rinck, Becker, Kellerman, \& Roth, 2003): this task is adapted from a standard visual search task (Neisser, 1963, for a review, see Rabbitt, 1982). Participants are instructed to search for a target word in a matrix of words, consisting of distracter words of one emotional or neutral category. Presence or absence of the target word should be indicated by a key press. Effects of interest are (1) selective enhancement of the target, indicated by speeded detection; (2) selective distraction by distracter stimuli, indicated by slower detection. Dot probe task (Macleod, Mathews, \& Tata, 1986): this task is adapted from a spatial cueing paradigm (Posner, Nissen, \& Ogden, 1978). Two words or pictures of different emotional valence (e.g. one neutral and one depression-related) are presented together, side by side. Immediately following the word-pair, a probe appears at one of the positions previously occupied by a word. In a probe detection task participants are instructed to signal the presentation of the probe by pressing a key. In variations of this task, participants are instructed to indicate the position of the probe (probe position task) or the type of probe (probe classification task), by pressing one of two response buttons. In all cases, the rationale is that probes that appear at an attended location will be detected relatively fast.

Deployment-of-attention task (DOAT; Gotlib, McLachlan, \& Katz, 1988): this task is very similar to a dot-probe task. Two words of different emotional valence are presented together, side by side. Immediately following the word pair, two colored bars are presented simultaneously. Participants are misinformed that one of the bars is presented shortly before the other, and the instruction is to decide which bar was presented first. The rationale is that the bar at the attended position will be detected first.

Emotional modification of an exogenous cueing paradigm (e.g. Fox, Russo, Bowles, \& Dutton, 2001): this task is adapted from an exogenous cueing paradigm (Posner, 1980). One cue (emotional or neutral) is presented to the left or to the right of fixation. Immediately following the cue, a probe is presented (with equal probability) to the left or to the right. Effects of interest are the emotional modification of: (1) IOR as a reflection of maintained attention to the emotional words; (2) attentional engagement and disengagement that can be examined by comparing emotional and neutral valid and invalid cues, respectively.

was moved to the upper position and this may have had an influence on attentional bias. To measure the distribution of attention in space in an unbiased way, an instruction that asks subjects to fixate at the center of the screen is preferred. Two additional changes that have been made in follow-up versions of the dot probe task, as in other tasks that have been used to measure attentional bias, were:

1. The use of different word types (a) to study specificity of attentional bias for different types of negative words (e.g. threat-related words and depression-relevant words), and (b) to examine if the bias was not a reflection of a general emotionality effect, by including words with a positive valence;

2. The use of different word exposure durations. This was done (a) to discriminate automatic from strategic bias processes, by using subliminal and supraliminal exposure 
durations, respectively, and (b) to verify the time course of attention, for example by using three different SOAs of increasing duration.

From Table 1.2 it can be derived that after the early failures (Macleod et al., 1986; Hill \& Duiton, 1989), an attentional bias in depression and dysphoria was demonstrated in a number of studies. For dysphoria, a bias was shown for depression-relevant words (Bradley, Mogg, \& Lee, 1997). Two dot probe experiments were conducted in which depression-relevant, anxiety-relevant and neutral words were used. Word pairs were presented at short (14 ms), medium (500 ms), and long (1000 ms) exposure intervals. In the first experiment, induced dysphoria was shown to be related to a bias for depression-relevant words in the $500 \mathrm{~ms}$ exposure condition, and to a lesser extent in the 1000 ms condition. In the second experiment, a positive relation was demonstrated between naturally occurring dysphoria and a bias for negative words (i.e. a combination of anxiety and depression words) in the 1000 ms exposure condition. In addition to a bias for depression-relevant words, dysphoria has been shown to be related to a bias away from happy faces (Bradley, Mogg, Millar et al., 1997; Bradley, Mogg, Falla, \& Hamilton, 1998). Furthermore, a protective bias, away from threatening faces and negative words, has been shown in nondysphorics in comparison to dysphorics (Bradley, Mogg, Millar et al., 1997; McCabe \& Toman, 2000). For depression, an attentional bias for threat words and negative words was demonstrated (Mogg, Bradley, \& Williams, 1995; Mathews, Ridgeways, \& Williamson, 1996). In both studies short (14 ms, $50 \mathrm{~ms}$ ) and long (500 ms, $1000 \mathrm{~ms}$ ) exposure durations were used and biases for depression were only demonstrated for the long duration. In two other dot probe studies in which faces were presented for $1000 \mathrm{~ms}$, an attentional bias for sad faces but not for threatening faces was shown in depressed participants (Gotlib, Kasch, Traill, Joormann, Arnow, \& Johnson, 2004; Gotlib, Krasnoperova, Neubauer, \& Joormann, 2004). In a number of studies that employed the DOAT, depressed showed an unbiased pattern of attention, while nondepressed showed a bias for positive words (Gotlib, McLachlan, \& Katz, 1988) or a protective bias away from negative words (McCabe \& Gotlib, 1995). Finally, in two studies that employed a visual search task, (Suslow et al., 2001; Suslow, Dannlowski, Lalee-Mentzel, Donges, Arolt, \& Kersting, 2004) depressed individuals were slower in detecting a positive face than controls.

In sum, although results have not been always replicated completely across studies, there is evidence for a selective bias for negative information in depression and dysphoria. In addition, a bias for positive words or a protective bias has been shown for control participants in some studies. The latter is in line with ideas that depressed have lost a positive bias or protective bias and suffer from "depressive realism" (Alloy \& Abramson, 1988; Gotlib et al., 1988; Taylor \& Brown, 1988).

As there was no evidence for a preconscious bias in dysphoria, Bradley, Mogg, and Lee (1997) concluded that attentional bias in dysphoria and depression may occur later in the information processing system and might be characterized by a difficulty to disengage from negative material that has come into the focus of attention. Some evidence for this comes from a study by Koster and colleagues (2005) that used an emotional modification of an exogenous cueing paradigm. An increased validity effect for negative words (presented for $1500 \mathrm{~ms}$ ) in dysphorics was due tot impaired disengagement. Furthermore, using a similar paradigm, Ellenbogen and colleagues showed that dysphorics in comparison to low-scorers on the BDI were slow to disengage atten- 
tion from negative, positive, and neutral words following a negative MIP (Ellenbogen, Schwartzman, Stewart, \& Walker, 2002). Also in line with this, in a visual search task there was no evidence for enhanced detection, but depressed participants were more distracted by depression-relevant words (Rinck \& Becker, 2005).

\section{Attentional bias in bipolar disorder}

Although there are a large number of studies on cognitive biases in major depression (MD) and dysphoria, little research has been conducted into the role of cognitive biases in bipolar disorder. In fact, when the discussion is limited again to selective attentional biases, no studies have been conducted in bipolar disorder patients yet. Bipolar disorders are characterized by episodes of remission, depression, mania, and mixed episodes (Diagnostic and Statistical Manual of Mental Disorders; 4th ed. (DSM-IV); American Psychiatric Association, 1994). Research has shown that transitions between the different episodes in bipolar disorders go together with changes in mood and cognitive functioning (Cuellar, Johnson, \& Winters, 2005; Murphy \& Sahakian, 2001). Episodes of mania and mixed episodes discriminate bipolar disorders from unipolar mood disorders. However, although bipolar disorder is often characterised by the elevated mood of mania and hypomania, depression appears to be the dominant affect (Judd et al., 2002; Judd et al., 2003; Mitchell \& Mahli, 2004). In addition, there are differences between bipolar depression and unipolar depression (for reviews, see Mitchell \& Mahli, 2004; Cuellar, Johnson, \& Winters, 2005; Mansell, Colom, \& Scott, 2005).

Given these differences between the disorders, and the advances that have been made in understanding unipolar depression, research on cognitive biases in bipolar disorders seems not only valuable but also essential. Since mania is usually regarded as a state that exists at the opposite end of depression on a spectrum of affect, it is interesting to examine if attentional biases are mood state dependent. Alternatively, or perhaps in addition, attentional biases might act as trait characteristics, similar in patient groups that vary in mood state, but different from healthy controls.

\section{General orienting processes and negative mood}

The idea that attentional biases in depression and dysphoria are related to processes of disengagement (Bradley et al., 1997) elicits the question if core deficits in attentional control may characterize depression. These problems may not only be related to negative mood through attentional bias, as it has been proposed that abilities to efficiently disengage from and shift attention to any object represent critical dimensions of self-regulatory behavior and effective coping (Derryberry \& Rothbart, 1988; Derryberry \& Rothbart, 1997; Derryberry \& Reed, 2002). In a nonclinical sample, results from questionnaire-data showed an inverse relation between the ability to shift attention and negative affect (Derryberry \& Rothbart, 1988). Therefore it was proposed that the capacity to control attentional orienting may play a role in negative affect. Similarly, those participants from a nonclinical sample who had difficulty to disengage their attention on an attentional orienting task showed the largest increase in negative affect in response to a distressing film (Compton, 2000). It was reasoned that an inability to disengage attention from the distressing material in the film may have caused a larger increase in negative affect. Mathews and Mackintosh (1998) proposed a relation be- 
tween attentional control and attentional bias. More specifically, they proposed that attentional bias would be strongest in those unable to control their attention. Derryberry and Reed (2002) found support for this claim as they showed that trait-anxious individuals with good attentional control were able to reduce their threat-related attentional bias and concluded that the magnitude and time course of attentional biases may always depend on the capacity to control attention.

In sum, these studies show that it is important to examine the ability to control attention in depressed and dysphoric individuals to find out if they have lower attentional control than healthy people.

\section{The time course of attentional bias}

The discrepancy of attentional bias results for depression and dysphoria may be related to the time course of attentional bias. Response times only provide a snapshot of attention. In a dot probe task, this snapshot is taken when the probe is presented. Response times therefore not necessarily reflect the first shift of attention toward or away from emotional stimuli. To get a better grip on attention and demonstrate its time course, some studies have used a range of SOAs (e.g. Bradley et al., 1997). An alternative to multiple SOAs is the use of measures that continuously monitor the distribution of attention. As argued above (paragraphs $1.4,1.5$ ) lateralized ERP components and lateralized changes in EEG frequency bands may be used for this goal. In addition, eye movements can serve as a continuous measure of overt attention.

To our knowledge, there are no studies that examined the time course of attentional bias for emotional stimuli with event-related changes in frequency bands, and studies that used ERP measurement for this purpose are scarce and limited to healthy (Pourtois, Grandjean, Sander, \& Vuilleumier, 2004; Stormark, Nordby, \& Hughdahl, 1995), or anxious (Bar-Haim, Lamy, \& Glickman, 2005) participants. In these ERP-studies, emotional stimuli functioned as exogenous cues (Stormark et al.) or were presented at fixation (Bar-Haim et al.). Although a dot probe task was used in the study by Pourtois et al. to examine attentional bias for fearful faces in healthy participants, they used ERPs for another purpose, to study modulatory processes of attention on the target.

Eye movements (EMs) are used more and more to measure the time course of attentional bias, especially in anxiety research (e.g. Hermans, Vansteenwegen, \& Eelen, 1999; Rohner, 2002; Pflugshaupt, Mosimann, Von Wartburg, Schmitt, Nyffeler, \& Müri, 2005; Rinck \& Becker, 2006). These measures have proven very useful in studies on anxiety to demonstrate vigilance-avoidance patterns of attention. Even with a combination of SOAs, these switches of attention could easily have been missed if only RTs would have been measured. In a small number of studies, EMs have been used to examine attentional bias for emotional stimuli in depression and dysphoria. This has been done both during (passive-) viewing tasks (Eizenman et al., 2003; Matthews \& Antes, 1992) and in a dot probe paradigm (Mogg, Millar, \& Bradley, 2000). When instructed to passively view a number of slides that each consisted of a social theme, a threatening theme, a dysphoric theme, and a neutral theme, depressed subjects elaborated longer, but not more frequently, on the dysphoric themes relative to control subjects (Eizenman et al., 2003). When presented with pictures consisting of happy and sad regions, dysphorics and control subjects fixated happy regions more often, sooner, and longer than sad 
regions (Matthews \& Antes, 1992). In addition, dysphorics fixated sad regions more often than control subjects. In a dot probe position task, there was no evidence that depressed subjects fixated sooner or more often on sad faces or away from happy faces than controls (Mogg, Millar, \& Bradley, 2000).

In sum, the promising results in anxiety research show the importance of eye movement measurement in studies of attentional bias. Measurement of eye movements may similarly provide more insights in the time course of attentional bias in depression and dysphoria. Lateralized ERP components and lateralized changes in EEG frequency bands measured time-locked to the onset of word stimuli in a dot probe task have not been used before to study the time course of attentional bias. Together, these measures may provide new windows on the time course of attentional bias in depression and dysphoria.

\section{Outline of the thesis}

The first three chapters (chapter 2, 3, and 4) that follow on this introduction will focus on EEG indices and response time measures of spatial orienting. Chapter 5 and 6 will be about attentional bias. More specifically,

- In chapter 2 , in a classic endogenous spatial cueing paradigm, processes of attention were examined in an analysis of EEG and RTs. The main aim was to examine processes of spatial attention in situations where attention is not explicitly directed by a cue, in neutral trials. Therefore, EEG correlates sensitive to attentional orienting were first derived from (left and right) cued trials. These included lateralized ERP components and lateralized changes in EEG frequency bands. A new method was developed that could be used to classify trials in one of two categories based on their single-trial event-related (lateralized) response in the EEG. Here, the method was used to classify EEG responses in neutral trials as "attention to the left" or "attention to the right". Response times were necessary to evaluate the classification and find out if attention was oriented in neutral trials. In addition to spatial attention, processes of general alertness in neutral trials were studied by analyzing the relation of RT and EEG correlates of general alertness. The latter consisted of nonlateralized ERP components and nonlateralized changes in EEG frequency bands. Analyses of EEG correlates of spatial attention in this chapter were important for following chapters. Successful classification of neutral trials in this chapter would indicate the validity and usefulness of these lateralized measures to serve as indices of orienting. In chapter 3 these indices of orienting were further analyzed and in chapter 6 they were used for applied purposes.

- In spatial cueing studies a sequence of lateralized ERP components related to processes of orienting has repeatedly been reported. However, the exact functional significance of these components is largely unknown. In chapter 3 , the functional significance of these ERP components was studied in two spatial cueing experiments. A simple detection task was used in experiment 1, and a go/ nogo discrimination experiment was used in experiment 2. More specifically, the goal was to discriminate components related to sensory aspects of the cue and components related to shifts of attention. Therefore, cue shape was manipulated in two tasks. In one of the tasks the physical properties of arrow stimuli were balanced across attention-left and attentionright conditions. Nonlateralized cues were used in the other task. 
- In most reaction time studies, only average results are reported, but research on sequence effects has shown that the effect of individual trials is influenced by preceding trials. Chapter 4 aimed to investigate whether orienting is sensitive to orienting processes in the previous trial in an endogenous spatial cueing paradigm. The effect of the previous cue, the previous target, and the previous trial type (i.e. validity) were examined.

- Although attentional biases for emotional stimuli have repeatedly been studied in unipolar depression and dysphoria, no studies have been conducted on attentional bias in bipolar disorder. The first aim of chapter 5 was to examine for the first time attentional bias in bipolar disorder. The second aim was to study general orienting processes and their relation to negative mood in bipolar disorder. A new paradigm was developed that combined an endogenous spatial cueing paradigm with a dot-probe paradigm.

- The empirical evidence regarding attentional bias in depression and dysphoria is heterogeneous. One of the reasons for discrepant results may be that attentional bias varies over time. Therefore, the time course of attentional bias was studied in chapter 6 . The paradigm developed in chapter 5 was adapted to study attentional bias in induced and naturally-occurring dysphoria. In addition to response time measures, eye movements and EEG were measured to continuously monitor how attention was distributed. Indices of orienting in the EEG were derived from chapter 2 . An additional aim of chapter 6 was to measure general orienting processes and their relation to negative mood in dysphoria. 


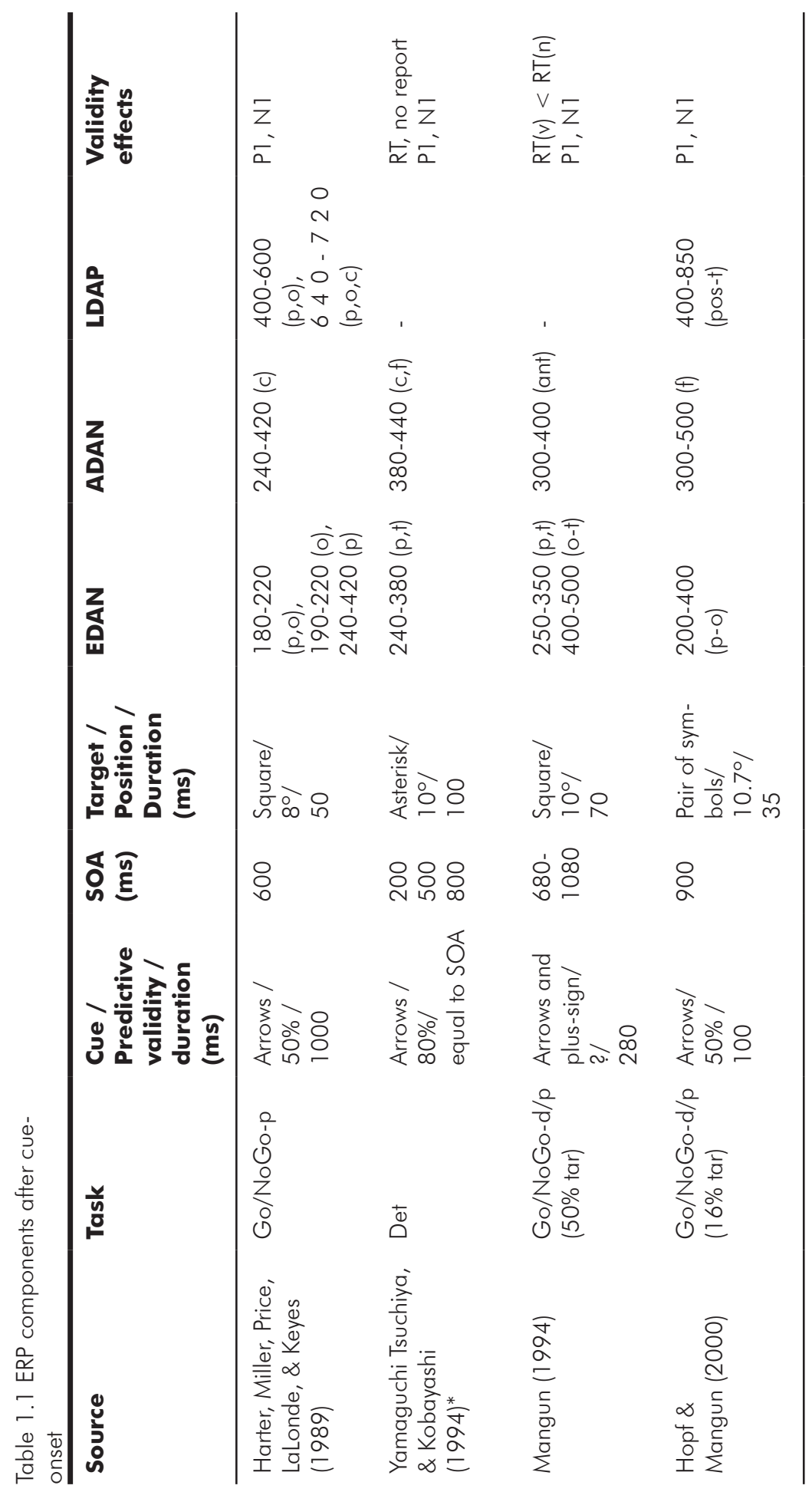




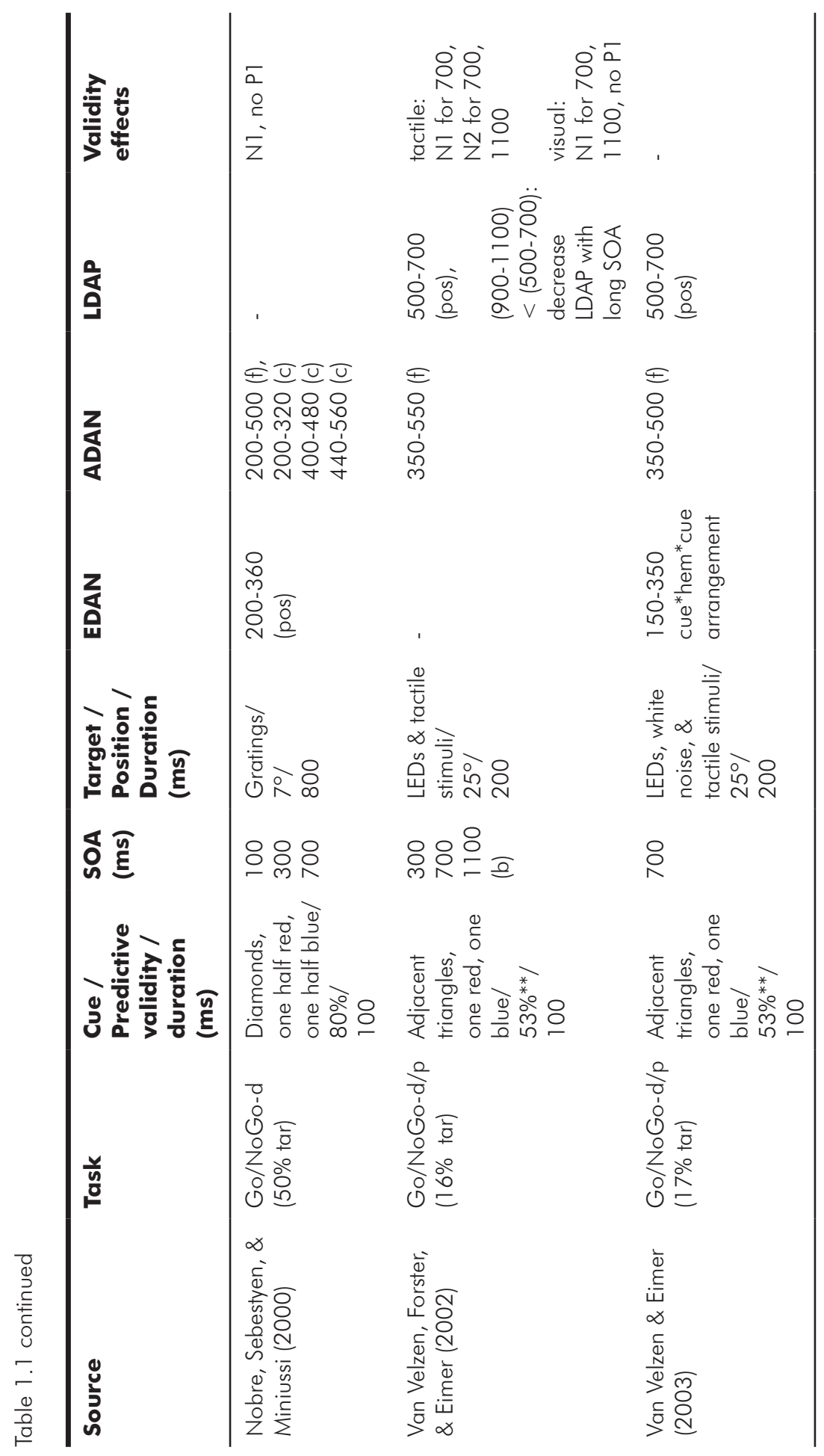




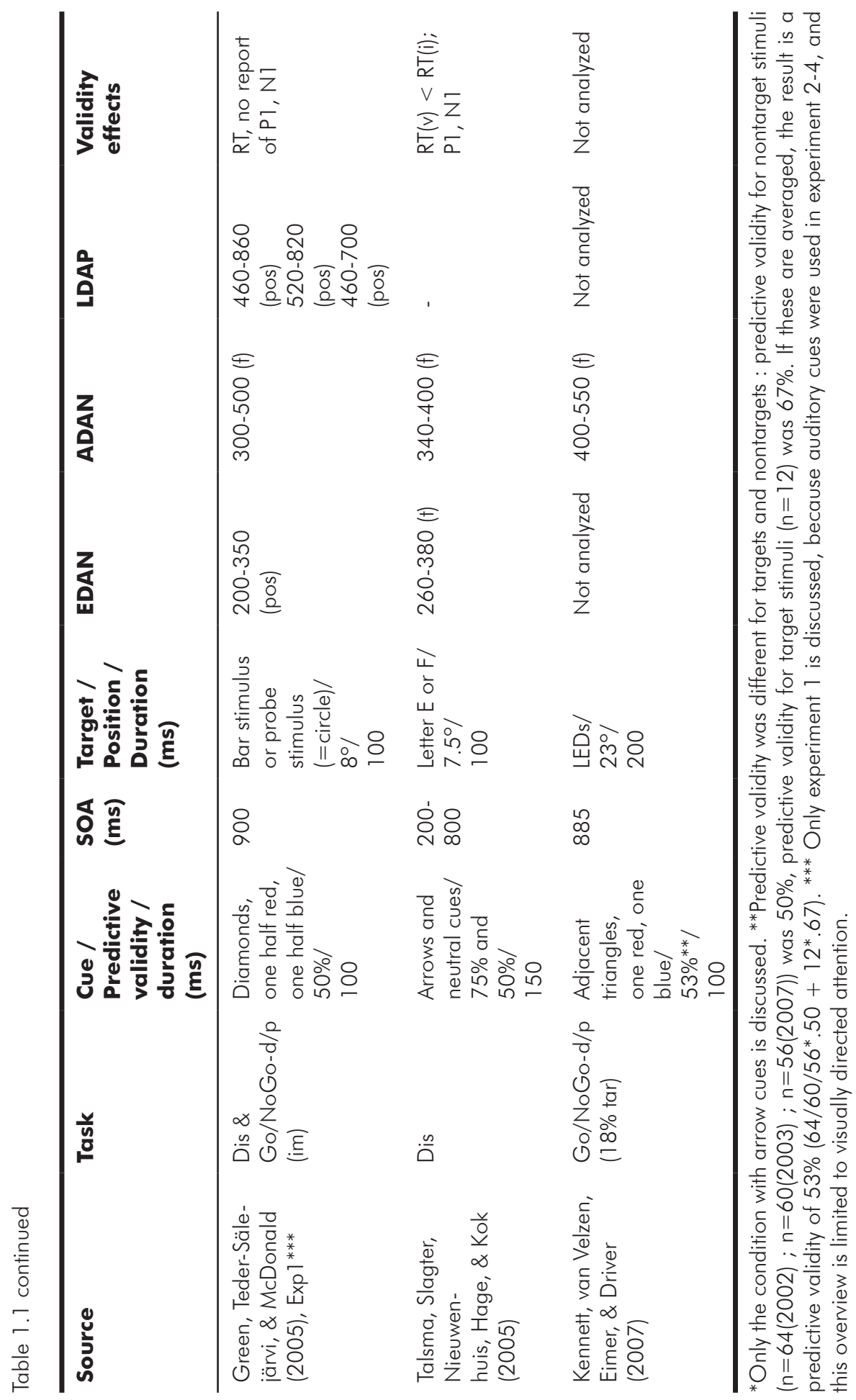




\section{Alphabetical list of abbreviations ( $p /$ column) in Table 1.1:}

Task

Det, detection task; detection of a target stimulus by pressing a response button as fast as possible

Dis, discrimination task; choice response to two types of target stimuli, presented at cued and at uncued positions, two response buttons

Go/NoGo-d, go/nogo discrimination task; responses only to target stimuli, presented at cued and at uncued positions, one response button

Go/NoGo-p, go/nogo position task; responses only to stimuli at cued positions, one response button

Go/NoGo-d/p, go/nogo discrimination/position task; responses only to target stimuli, only when presented at cued positions, one response button

im, intermixed

tar, target stimuli

EDAN / ADAN / LDAP

ant, anterior

f, frontal

0, occipital

o-c, occipito-temporal

p, parietal

$\mathbf{p}-\mathbf{0}$, parieto-occipital

pos, posterior

pos-t, posterior-temporal

t, temporal

Validity effect

i, invalid

n, neutral

$\mathbf{R T}$, response times

$\mathbf{V}$, valid 


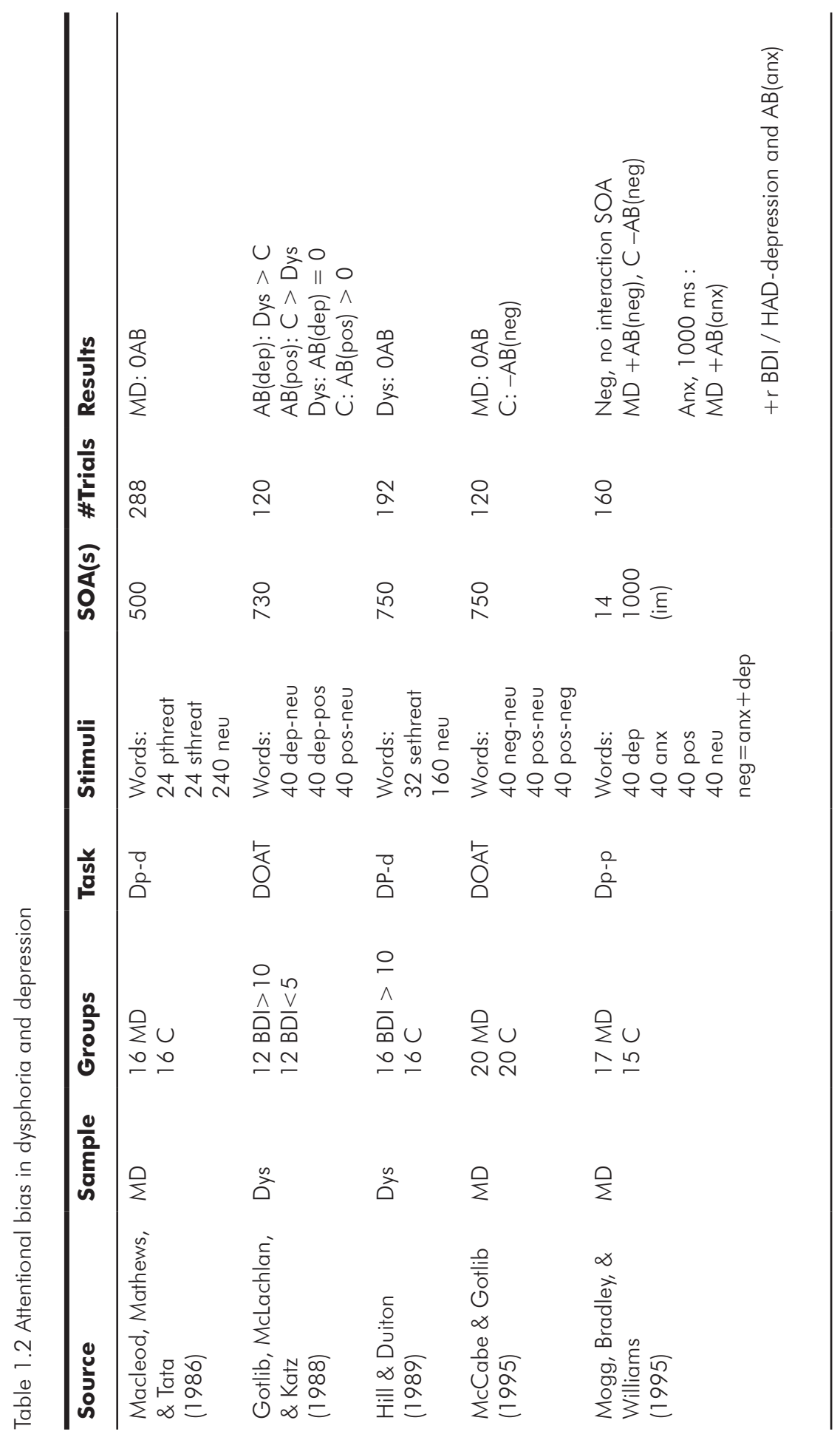




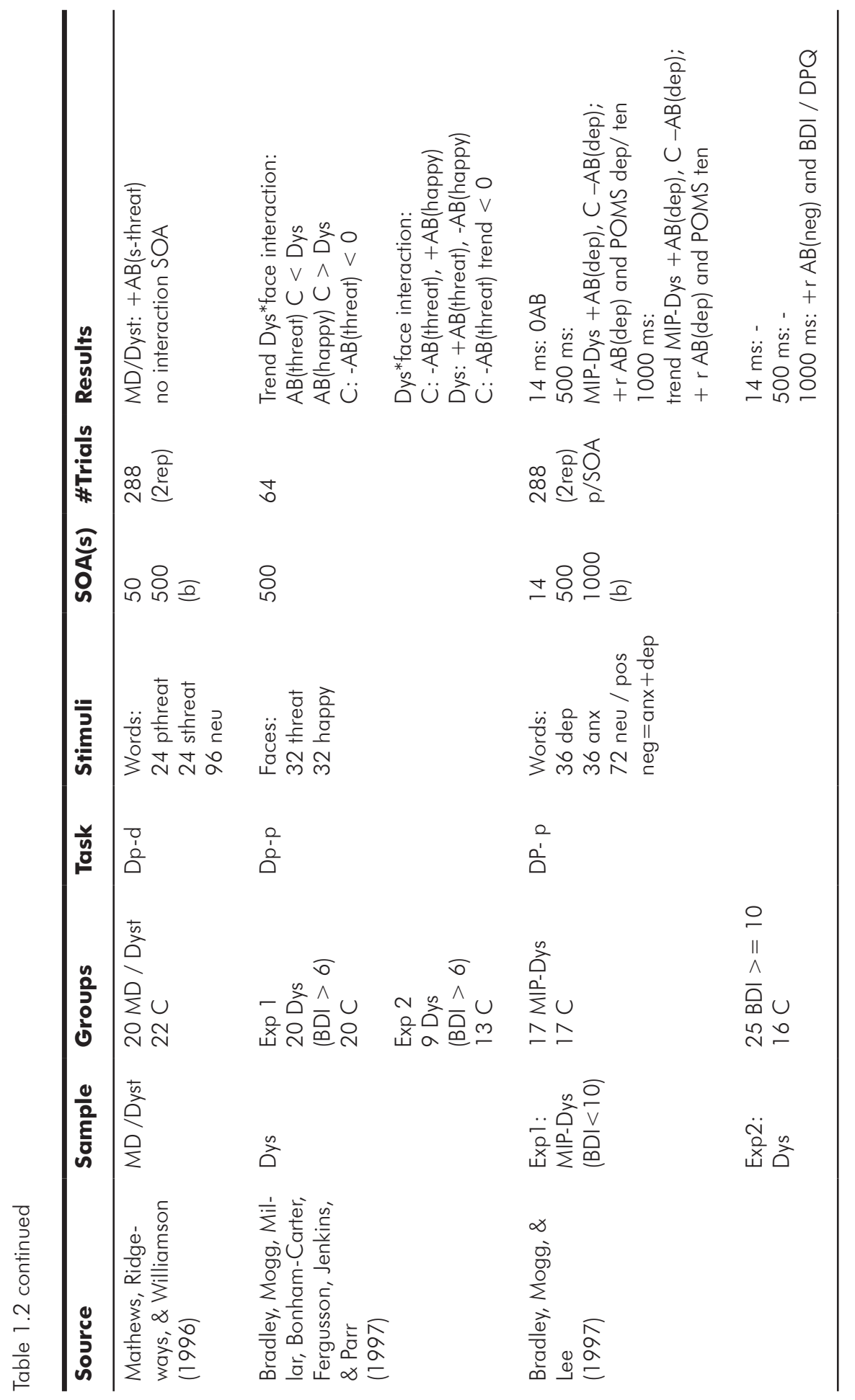




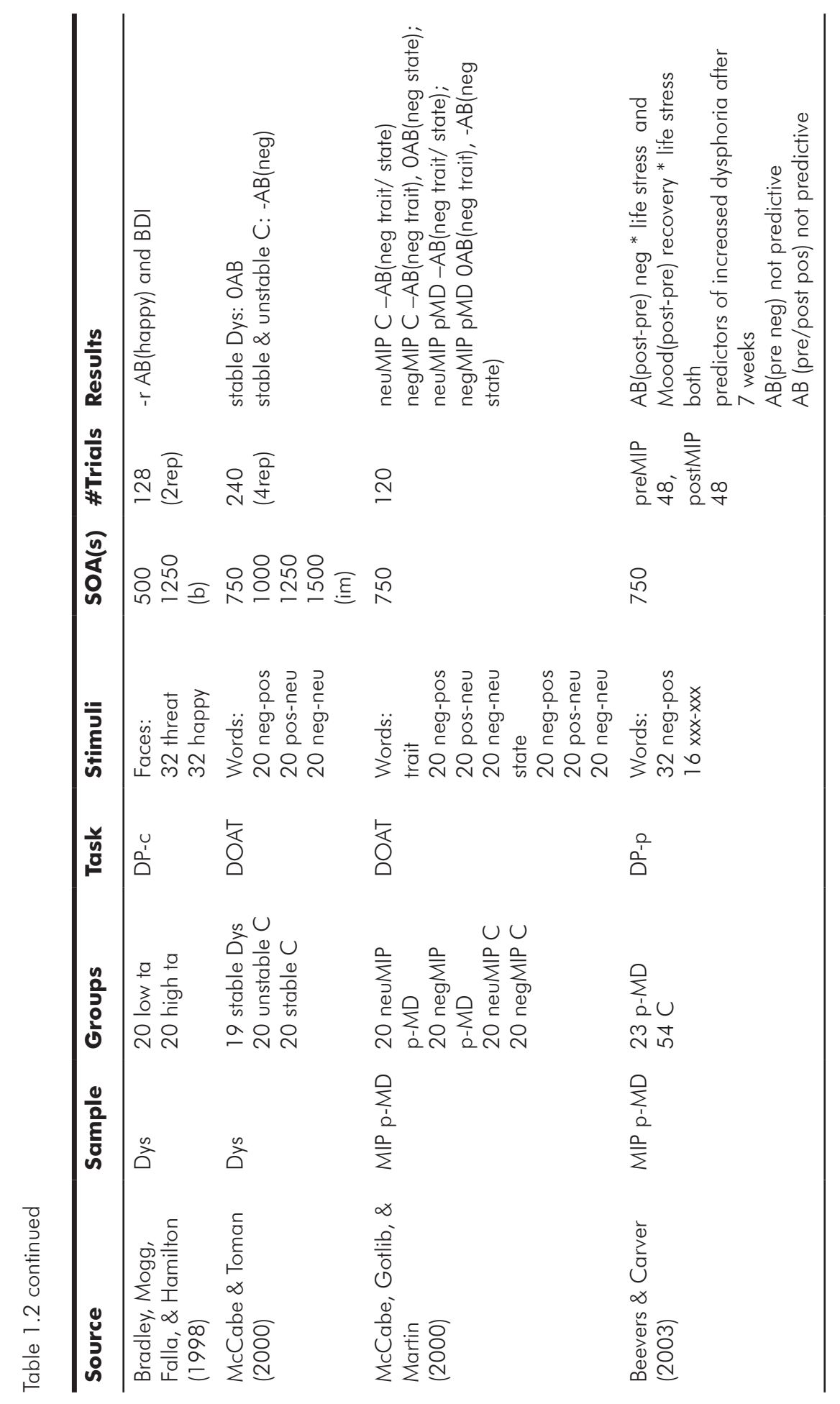




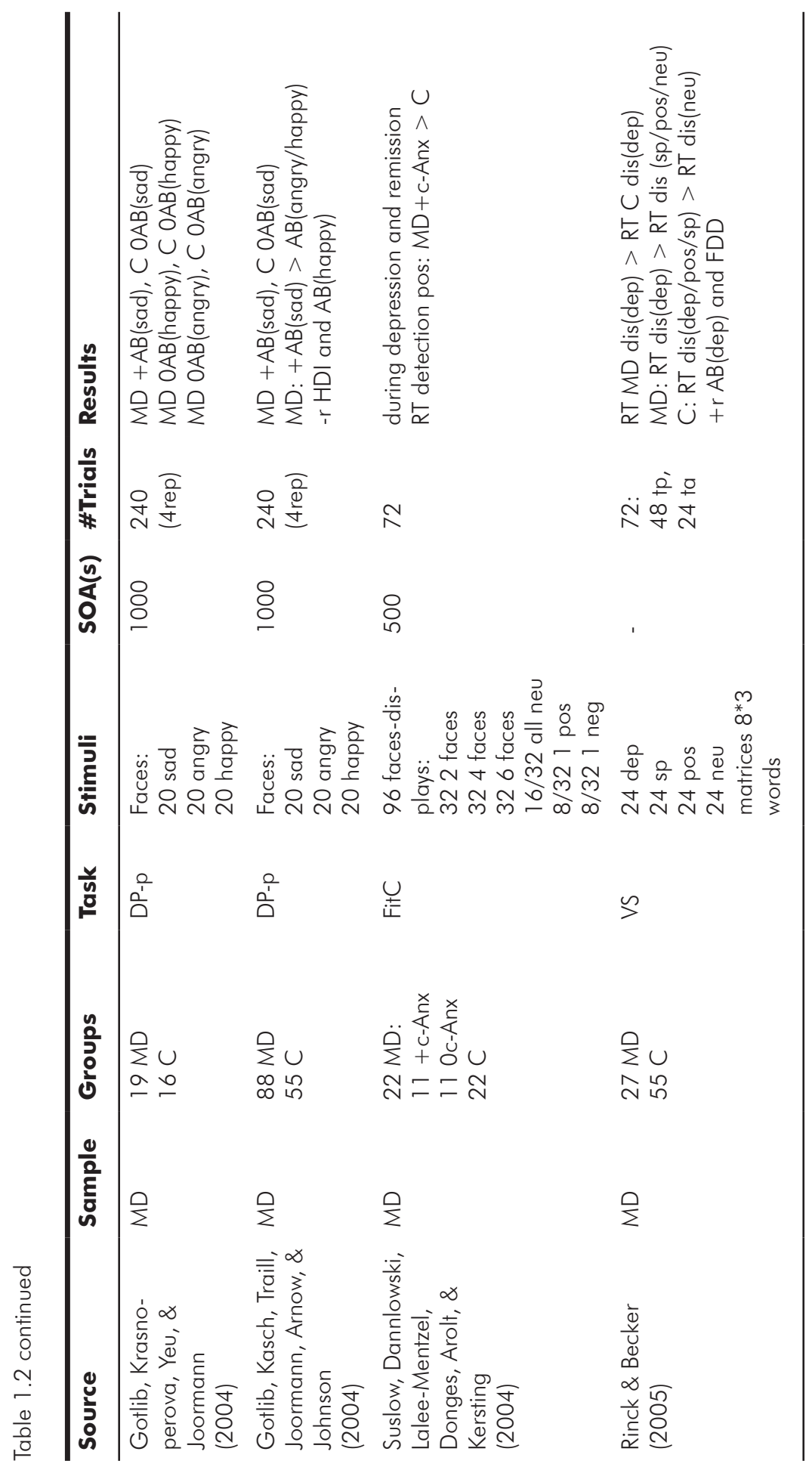




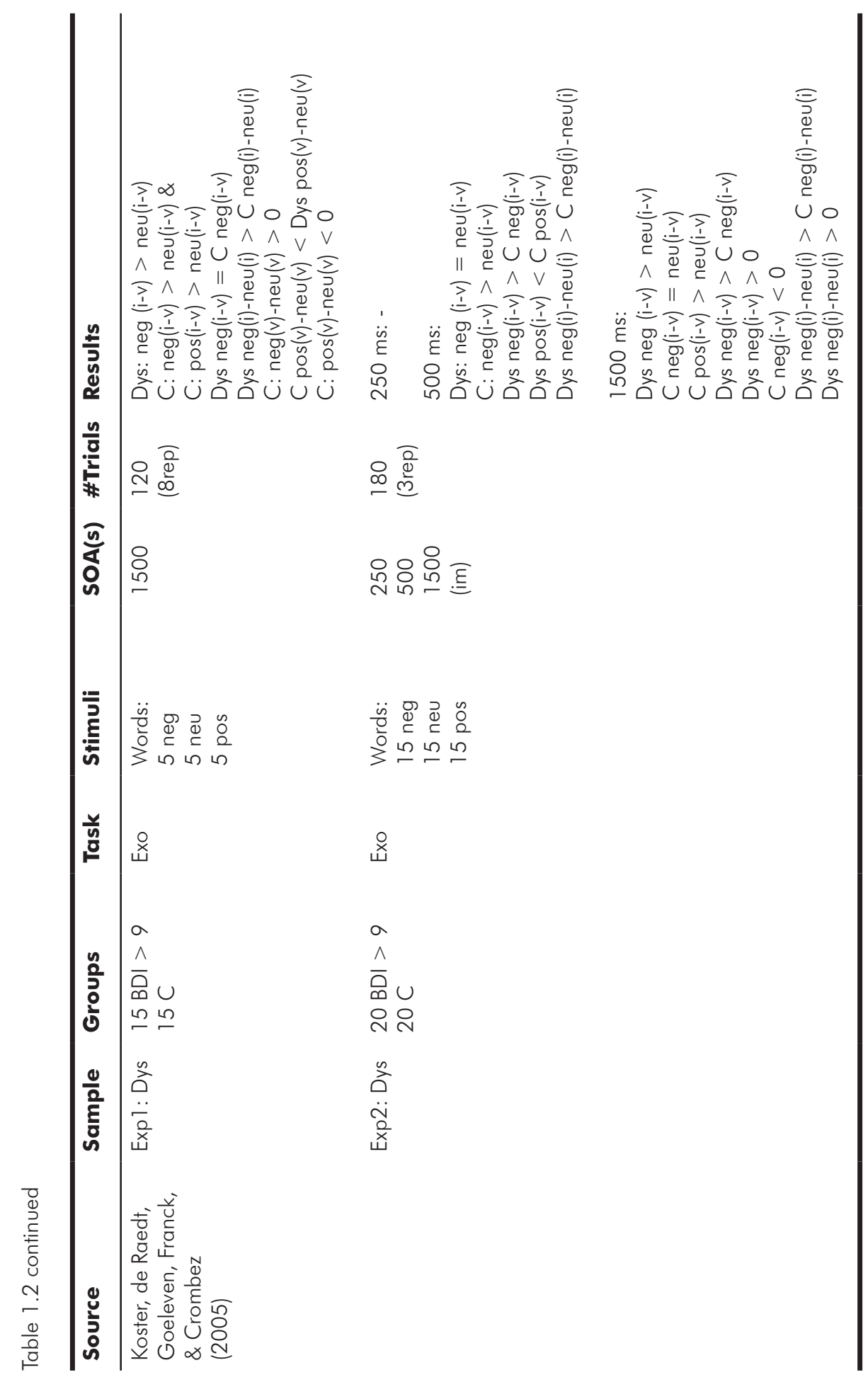




\section{Alphabetical list of abbreviations ( $p /$ column) in Table 1.2:}

\section{Sample / Groups}

BDI, Beck Depression Inventory

C, heathy controls

c-Anx, comorbid anxiety disorder

Dys, dysphorics

exp, experiment

MD, major depressed

MIP, mood induction procedure

MIP-dys, mood induced dysphoria

p-MD, previously depressed

ta, trait anxious
Task

DOAT, Deployment-of-Attention task DP-c, dot probe classification task

DP-d, dot probe detection task

Dyst, dysthymia

Exo, emotional exogenous cueing task

FitC, Face-in-the-Crowd

VS, visual search task

\section{Stimuli}

anx/dep, anxiety-relevant or depression-relevant stimuli

anx, anxiety-relevant stimuli

dep, depression-relevant stimuli

dis, distracter stimuli

neg, negative stimuli

neg-neu, pairs of negative and neutral stimuli

neg-pos, pairs of negative and positive stimuli

neu, neutral stimuli

neu/pos, neutral or positive stimuli

pos-neu, pairs of positive and neutral stimuli;

pthreat, physical threat

SP, social phobia relevant stimuli

sthreat, social threat

sethreat, self esteem threat

SOA

b, blocked

im, inter-mixed

Results
\#Trials

rep, number of repetitions $\mathrm{p} /$ stimulus

ta, target absent

tp, target present

AB, attentional bias

DPQ, Depression Proneness Questionnaire

FDD, German version of the Questionnaire for Depression Diagnosis

i, invalid

POMS, Profile of Mood States

POMSdep, POMS depression subscale

POMSten, POMS tension subscale

$\mathbf{r}$, correlation

RT, response time

$\mathbf{v}$, valid

-, negative / away from

$\boldsymbol{+}$, positive / toward

$\mathbf{0}$, no 
80
$80 c$
occ chapter 1 


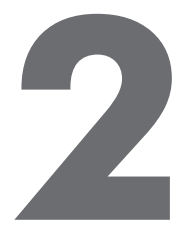

\title{
Varieties of attention in neutral trials:
}

\author{
Linking RT to ERPs and EEG \\ frequencies
}

It is often assumed that when a neutral cue is presented in a spatial cueing task, attention remains at fixation until target onset. We hypothesized that variance in nonspatial attention and switches of attention towards target locations can account for variance in reaction times (RTs) of neutral trials. Lateralized event-related potentials (ERPs) and changes in EEG frequency bands served as predictor variables in a single-trial logistic regression analysis to predict the direction of spatial attention in cued and neutral trials. The contingent negative variation (CNV) and nonlateralized changes in the alpha band served as markers of nonspatial attention. The direction of attention in cued trials was reliably predicted from single trial lateralized ERP components. In neutral trials, only evidence for nonspatial attention was found, indicated by increases in the CNV and decreases in alpha preceding targets to which responses were relatively fast.

Jongen, E.M.M., Smulders, F.T.Y., \& Van Breukelen, G.J.P. (2006). Varieties of attention in neutral trials: Linking RT to ERPs and EEG frequencies, Psychophysiology, 43, 113-125. 
Spatial attentional orienting in the visual domain has often been studied by cueing paradigms in which a cue predicts the likely location of a target stimulus. Typically, reaction times (RTs) are faster to validly than to invalidly cued targets and RTs of neutral trials are in between (e.g. Posner, Nissen, \& Ogden, 1978). These effects are interpreted as the result of shifts of attention that follow the cue. This has been supported by electrophysiological (see for a review Luck, Woodman, \& Vogel, 2000) and imaging studies (see for a review Corbetta \& Shulman, 2002). However, very few studies have examined cognitive processes that follow the presentation of neutral cues.

Posner (1978) argued that attention will remain at fixation after a neutral cue, because it has no predictive validity and therefore there is no advantage to orient attention towards any of the target locations. Evidence opposing Posner's idea was obtained by De Gonzaga Gawryszewski, Riggio, Rizzolatti, \& Umiltá (1987) using a detection task in which targets occasionally appeared at fixation. When the fixation position was cued, RTs to peripheral stimuli were much longer than in the conventional neutral condition when targets always appeared at peripheral positions and never at fixation. Therefore, the authors proposed some alternative possibilities of attentional processing following a neutral cue. First, it could be that attention is switched randomly or systematically between the locations where targets may appear. Second, instead of a focused state of attention, neutral trials might be characterized by the diffuse state of attention that was postulated by Jonides (1983). Third, following the terminology of the multistage model by Posner (1978), attention might be disengaged and ready to move after a neutral cue is presented.

The aim of the present study was to use effects of attention on the electroencephalogram (EEG) to examine processes of attention in neutral trials. These processes can be divided into at least two classes. Posner and Boies (1971) discriminated processes of attention that heighten general alertness and processes that selectively prepare a subject, for example for a location. While spatial cueing paradigms have been used to study processes of selective attention for location, the increase in general alertness has been addressed by experiments that used warning signals (e.g. Bertelson, 1967). These experiments showed that performance is improved when a warning signal is given and target stimuli appear at a predictable interval. Since a neutral cue can serve as a warning signal, it is hypothesized that general alertness, or nonspatial attention, may increase when a neutral cue is presented and that variations in nonspatial attention will lead to variations in behavioral performance. Regarding selective spatial attentional processing in the neutral trials, we will specifically examine one alternative proposed by De Gonzaga Gawryszewski et al. (1987) that after a neutral cue is presented, attention is switched, randomly or systematically, to the locations where targets can appear. EEG recordings during an endogenous cueing task were used to provide continuous and online measures of attention shifting. Both nonspatial and spatial attentional processing have already been studied with EEG.

Nonspatial attention effects on EEG. Walter, Cooper, Aldridge, McCallum and Winter (1964) reported a negative slow potential, (contingent negative variation, $\mathrm{CNV}$, or "expectancy wave") during the foreperiod of a RT task. Across trials, an increase in the amplitude of this component was related to a reduction of RT (see also Hackley and Miller, 1995). Brunia and Damen (1988) could discriminate preparation for movement and preparation for the stimulus, both characterized by a negative wave (for a 
review, see Brunia, \& van Boxtel, 2001). Miniussi et al. (1999) showed that the CNV is increased when faster orienting is required by the task. Finally, the CNV component differs between responses that have to be performed and responses that have to be withheld (Walter et al., 1964). In all, it appears that the CNV may be useful as an index of nonspatial attention in a RT task.

Nonspatial attention effects have not only been related to ERPs, but also to changes in neural oscillations, reflected in the EEG. These oscillations can be quantified by computation of event related desynchronization (ERD) or event related synchronization (ERS) (e.g. Pfurtscheller \& Aranibar, 1977; Kalcher \& Pfurtscheller, 1995) or temporal spectral evolution (TSE, Salmelin \& Hari, 1994). Several studies have shown that decreases in the alpha band represent a correlate of activated cortical areas (e.g. Klimesch, 1999; Klimesch et al., 1996), and increases in alpha correspond to decreased functional activity in the underlying cortex (e.g. Pfurtscheller, 1992; Pfurtscheller, Stancák, \& Neuper, 1996). The latter was recently supported by an fMRI study (Goldman, Stern, Engel, \& Cohen, 2002) that found increased alpha to be correlated with decreased activity in multiple regions of the cortex. Decreases in alpha have been related to increases in attention and activation (e.g. Boiten, Sergeant, \& Geuze, 1992; Dujardin et al., 1993; Vazquez Marrufo, Vaquero, Cardoso, \& Gómez, 2001) and it has been shown that decreases in alpha are related to stimulus anticipation (Bastiaansen \& Brunia, 2001; Filipovic, Jahanshahi, \& Rothwell, 2001; Bastiaansen, Böcker, \& Brunia, 2002). For these reasons, changes in the alpha frequency band during the cue-target interval will be measured, and added to CNV data to examine if variations in nonspatial attention can explain part of the variance in RTs of neutral trials.

Spatial attention effects on EEG. Harter, Miller, Price, LaLonde and Keyes (1989) were the first to describe ERP components in the cue-target interval related to shifts of visuo-spatial attention. The earliest component ("early directing attention negativity", EDAN), was a negative deflection over the hemisphere contralateral to the direction of the endogenous cue, maximal at parietal sites, starting $200 \mathrm{~ms}$ after cue onset and lasting about $200 \mathrm{~ms}$. The second effect, also posterior sites, between 400 and $700 \mathrm{~ms}$, was a contralateral positivity ("late directing attention positivity", LDAP). It was hypothesized that the early directing attention negativity is related to the processing of the meaning of the cue and the direction of attention, and that late directing attention positivity is related to preparation for the target. Many studies have since reported these ERP components (e.g. Nobre, Sebestyen, \& Miniussi, 2000; Hopf \& Mangun, 2000), although some failed to find the late directing attention positivity (Harter, \& Anllo-Vento, 1991; Mangun, 1994; Yamaguchi, Tsuchiya, \& Kobayashi, 1994) or the early directing attention negativity (Eimer \& Van Velzen, 2002; Eimer, Van Velzen, \& Driver, 2002). Several authors (Hopf \& Mangun, 2000; Mangun, 1994; Nobre et al., 2000; Van Velzen, Forster, \& Eimer, 2002) reported an additional negative component at anterior sites between 300 and $500 \mathrm{~ms}$, the anterior directing attention negativity (ADAN). Possibly, this component is related to the control and initiation of attentional shifts.

Spatial orienting of attention has also been related to changes in neural oscillations in the alpha and gamma frequency bands. An increase of alpha was found ipsilateral to stimuli that had to be attended and therefore contralateral to stimuli that had to be ignored (Worden, Foxe, Wang, \& Simpson, 2000). Comparable increases of alpha have also been shown in cross-modal cueing paradigms, where parieto-occipital alpha in- 
creased when the visual modality had to be ignored (Foxe, Simpson, \& Ahlfors, 1998; Fu et al., 2001). These increases of alpha have been interpreted as active gating of distractor stimuli and uncued spatial locations. In addition to alpha activity, gamma activity has been shown to be related to attentional cueing. It has been shown that gamma activity increases with high arousal, alertness and attention (Bouyer, Montarom, Vahnee, Albert, \& Rougeul, 1987; De Pascalis \& Ray, 1998; Müller, Gruber, \& Keil, 2000; Sheer, 1976; Shibata et al., 1999; Sokolov et al., 1999) and is related both to bottum-up and top-down attentional processing (for reviews see Muiller and Gruber, 2001; Keil, Gruber, \& Muiller, 2001). In an attentional orienting study, after the cue, gamma activity (35-51 $\mathrm{Hz}$ ) shifted from a broad posterior distribution to an increase of power at parieto-occipital sites, contralateral to the to-be-attended side (Gruber, Müller, Keil, \& Elbert, 1999). In sum, there is positive evidence that several components in the ERP and EEG can be used as sensitive indices of shifts of spatial attention.

The present study embodies an attempt to find evidence for the contribution of nonspatial and spatial attention during the cue-target interval to variations in RTs in neutral trials. Nonspatial attention was studied by examining the relation between RT and nonlateralized ERPs (CNV) and oscillations during the cue-target interval. Spatial orienting in neutral trials was examined in a more elaborate way. First, the lateralized EEG responses to directional cues were analysed to find out which variables (time periods in the cue-target interval, electrode positions) were sensitive to attentional orienting. In the second step, the (known) cue direction in cued trials was 'predicted' from these lateralized EEG responses in the cue-target interval. This was done for every subject, in a single trial logistic regression analysis. The successfulness of this 'prediction' of cue direction was evaluated by combining the 'predicted' cue direction with the actual target location, then sorting trials into 'predicted valid' and 'predicted invalid' categories, and analyzing RTs for 'predicted validity' effects. If these were significant, it was concluded that the direction of attention could be determined sufficiently reliably by only looking at the EEG during the cue-target interval. In that case, the predictor analysis was repeated in a third step where it was applied to neutral trials. If attention is really oriented in these trials, it should be possible to predict its direction above chance level. The successfulness of this prediction was again evaluated by combining predicted direction of attention and actual target location into an analysis of 'validity' effects on RT. If no spatial orienting occurs in neutral trials, the prediction of the direction of attention will be entirely random and the predicted validity effect on RT will be effectively zero. If, however, attention is switched, randomly or systematically, to the locations where targets can appear in a significant proportion of neutral trials, the prediction should be above chance level and predicted validity effects will be manifested in RT to the target.

\section{Method}

\section{Subjects}

Twenty-five healthy volunteers ( 23 female) between the ages of 18 and 31 years (mean age 22.3) participated in the study. All reported normal or corrected-to-normal vision. One participant was left-handed. The experimental methods received prior approval 
of the institutional ethics committee. All participants signed an informed consent to participate and received 25 euro.

\section{Stimuli, apparatus and procedure}

Subjects were comfortably seated in a dimly lit, sound attenuated room. The stimuli were presented on a VGA monitor at $100 \mathrm{~cm}$. Stimuli consisted of black-and-white, vertical square-wave gratings $\left(3.7^{\circ}, 4.4\right.$ (high spatial frequency) or 2.2 (low spatial frequency) cycles/degree) presented to the left or right $\left(5.4^{\circ}\right)$ of fixation on a gray background with the same average luminance as the gratings. ${ }^{1}$ The task was a detection task where every grating stimulus required a right-hand response, independent of its spatial frequency or location. Catch trials were added to prevent premature responses.

Each trial started with a fixation cross, which stayed on throughout the trial. After $100 \mathrm{~ms}$, a cue was presented for $600 \mathrm{~ms}$. In half of the trials, the cue was an arrow to the left $(<<)$ or to the right $(>>)$ that validly indicated the location of the subsequent target in $80 \%$ of the cases. In the other half, a neutral, double arrow cue $(<>)$ was presented that had no predictive validity. At $1200 \mathrm{~ms}$ after cue onset, the target was presented for $200 \mathrm{~ms}$. Subjects had to respond to the target by pressing a button with their right index finger. The intertrial-interval varied between 3900 and $4400 \mathrm{~ms}$.

The experiment consisted of a 100-trial practice block and a 360-trial (plus 8 warmup trials) experimental block. The proportion of valid : invalid : neutral : catch trials in the experimental block was $16: 4: 20: 5$. In the experimental block, subjects received feedback about their reaction time after every 20 trials and could then proceed with the task when they felt ready. After 180 trials, there was a ten-minute break.

Subjects were instructed to respond quickly and accurately and to maintain central eye fixation during the trials. Corrective feedback was given on misses and false alarms and on responses that preceded target onset, or were too fast $(<120 \mathrm{~ms})$ or too slow (> $2000 \mathrm{~ms})$.

To ensure central fixation during the task, horizontal EOG was recorded, continuously inspected and used for offline removal of trials that contained eye movements, because these trials were assumed not to reflect covert attention. To determine removal criteria, subjects received an eye movement calibration task in which the voltage-level corresponding to an eye movement to the left and to the right target locations was determined for every subject because horizontal EOG amplitude depends on head shape and exact electrode position.

Before the EEG cap was positioned, subjects first received the instruction of the spatial cueing task and performed on the practice block of the task. When the electrodes were positioned, the eye movement and blink calibration task were presented, followed by the experimental block of the spatial cueing task.

\section{Data recording}

The electroencephalographic (EEG) activity was recorded at 28 scalp locations (midline: Fz, FCz, Cz, Pz, Oz; lateral: FP1/FP2, F3/F4, F7/F8, FC5/FC6, C3/C4, CP5/CP6, P3/P4, P7/

1 The reason for including both high and low spatial frequency targets is not central to the current paper: it was merely done to replicate an earlier unpublished study done in our lab that had obtained good effects. 
P8, P9/P10, P07/P08, 01/O2; and right mastoid A2) using tin electrodes mounted on an elastic cap (Quik-Cap). A1 and A2 were used as reference and data were mathematically re-referenced offline (according to the average of A1 and A2). AFz was used as the ground. Tin electrodes were also used to bipolarly record the vertical (above and below the left eye) and horizontal (at outer canthi of both eyes) electrooculograms (EOGs). Electrode impedance was kept below $5 \mathrm{kOhm}$, amplifier bandpass was $0.05-100 \mathrm{~Hz}$, and digitisation rate was $500 \mathrm{~Hz}$.

Incorrect response trials and trials with an RT deviating more than 2.5 standard deviations from the mean, based on the sample distribution of every trial type (valid / invalid / neutral) for that subject, were excluded. Trials with eyeblinks were corrected with a regression procedure (Semlitsch, Anderer, Schuster, \& Presslich, 1986), using a blink correction factor derived from a separate blink calibration task in which "spontaneous" blinks were promoted by demanding constant fixation to detect slow color changes of the fixation cross.

Trials with an EEG voltage exceeding $100 \mu \mathrm{V}^{2}$ and trials with eye movements were excluded from analysis. During the eye calibration task, the HEOG amplitude was determined that resulted from an eye movement towards the left and right target location. Subsequently, the median amplitude was calculated separately for eye movements to the left and to the right (both 18 trials), and 30\% of this median was taken as a criterion for trial rejection in the spatial cueing task. This percentage was determined by examination of the averages of the HEOG channel for cues to the left and cues to the right. While weaker criteria left more trials for analysis, the criterion of $30 \%$ was needed for the average HEOG channel to not display any sign of eye movements anymore. In total, an average of $16 \%$ of trials were excluded (range: 6-38\%) .

ERPs and event-related changes in EEG frequency bands were analysed. For both analyses, the EEG was epoched into $1400 \mathrm{~ms}$ periods, starting $200 \mathrm{~ms}$ prior to the cue, and the $200 \mathrm{~ms}$ pre-cue interval was used as a baseline. For frequency band analyses, absolute amplitude changes were computed following the "temporal spectral evolution" method (Salmelin \& Hari, 1994). Alpha bands were defined similar to Doppelmayr (1998). The lower alpha1 $(6-8 \mathrm{~Hz})$, the lower alpha2 $(8-10 \mathrm{~Hz})$ and the upper alpha (10$12 \mathrm{~Hz}$ ) band were computed as well as an alpha band of $8-14 \mathrm{~Hz}$ that was similar to the band Worden et al. (2000) used. In addition, similar to Gruber et al. (1999) several "higher" frequency bands, often labeled "beta" or "gamma", were computed. These were the following: $15-18 \mathrm{~Hz}, 19-35 \mathrm{~Hz}, 36-51 \mathrm{~Hz}, 52-67 \mathrm{~Hz}, 68-83 \mathrm{~Hz}$. Bandpass filtering (Butterworth, zero phase, $96 \mathrm{~dB} / \mathrm{oct}$ ) was done at the continuous file, before data were epoched. After epoching, the filtered ERP was computed by averaging the data for every cue condition. Induced epochs were computed by subtracting the filtered ERP component for every cue condition from the filtered epoched data of the same condition. The purpose of computing induced changes (Klimesch, Russegger, Doppelmayr, \& Pachinger, 1998) is to discriminate phase-locked from non-phase-locked event-related EEG activity. After baseline correcting induced epochs for every condition with the pre-stimulus interval, data were rectified and, for the single trial analysis, smoothed with a moving average filter. The number of points in the moving average always cor-

2 In eight subjects this criterion was increased to $150 \mu \mathrm{V}$ because inspection of the EEG showed that large amounts of alpha activity caused increases of the voltages in their EEG. 
responded to a single period of the center frequency. For the average frequency band analyses, the rectified data were first averaged before they were smoothed.

\section{Data analysis}

Spatial attention. To investigate spatial orienting in neutral trials, an analysis was done in three steps. In the first step, attentional orienting effects on RT and EEG in the cue-target interval were analyzed. Specifically, a 3 (cue direction: left, right, neutral) $\times 2$ (target: left, right) $\times 2$ (spatial frequency: high, low) within-subject ANOVA was done on RT data to analyze effects of cue validity. For both lateralized ERP and lateralized frequency band data in the cue-target interval, successive mean voltage values every $50 \mathrm{~ms}$ were used as dependent variables in three separate regional analyses (anterior (F7/8, F3/4. FC5/6), central (C3/4), and posterior (P9/10, P7/8, P3/4, PO7/8, O1/2)). In these analyses, the following three factors were tested within subjects: cue direction (2: left, right), hemisphere (2: left, right) and electrode (number dependent on the region), and any interaction between cue and hemisphere was taken as a reflection of attentional shifting. Lateralised effects found to be affected by electrode (as indicated by interactions between cue direction, hemisphere and electrode) were assessed separately for the different electrodes in a region. The Greenhouse-Geisser epsilon correction factor was applied to compensate for possible effects of non-sphericity in the measurements compared. Only the corrected F- and probability values and the uncorrected degrees of freedom are reported. Because of multiple interrelated comparisons, and hence the likelihood of false-positive significant effects, effects were only considered reliable if they persisted for at least two successive time bins.

In the second step, ERP and frequency band lateralizations in the cue-target interval were used as predictor variables in two separate analyses, to 'predict' cue direction in the directionally cued trials. Time periods and electrode locations of significant lateralizations in the first step were chosen as predictor variables. Since the dependent variable was dichotomous (i.e. left, right), logistic regression was used. To test if noise levels were not too high for succesful categorization of single trials, a cross-validation procedure was followed. The odd numbered cued trials served to generate regression weights, one weight for every variable, that discriminated optimally between cue-left and cue-right trials. These weights were then used to 'predict' cue direction in the even numbered trials. This prediction resulted in an estimated probability for every even cued trial that was simplified to a classification of two categories ('left' or 'right'). To evaluate this classification, RT data of the even trials were submitted to two ANOVAs, one with 'actual cue direction' and 'target' as factors, and one with 'predicted cue direction' and 'target' as factors. If a significant interaction between these factors was found twice, it was concluded that it was possible to predict cue direction in a sample of cued trials, based purely on the EEG signal during the cue-target interval. In that case, there should be sufficient power to classify neutral trials in a similar way, at least if attention moves (randomly or systematically) in these trials. This prediction was done in the third step.

In the third step, logistic regression was again done per subject, but now to 'predict' the direction of attention for every neutral trial. Time periods and electrode locations of the predictor variables were similar to step 2 . Regression weights for the predictor variables of the neutral trials were derived from all cued trials to further increase 
reliability. Classification of predicted direction of attention was again evaluated in a 2 (predicted direction of attention: left, right) $\times 2$ (target: left, right) ANOVA on RT of all neutral trials. A significant interaction between these factors was taken both as an indication that the categorization was to some degree successful, and thus, as evidence that in neutral trials attention is switched to the locations where targets can appear.

Nonspatial attention. Nonspatial attentional processing in neutral trials was examined by analysing the relation of RT with the nonlateralized CNV component and nonlateralalized changes in the alpha band $(8-14 \mathrm{~Hz})$ in the cue-target interval of the neutral trials. Therefore, prior to signal averaging, neutral trials were sorted into three RT bins for both target positions. The fastest 33\% and the slowest 33\% RTs were examined. We hypothesized that a larger amplitude of the (negative) CNV component and a larger decrease in the alpha band would precede faster responses than slower responses. Following Hackley and Miller (1995), electrode positions C3, C4, Cz and Pz were examined from $400 \mathrm{~ms}$ before target onset until target onset.

\section{Results}

An alpha level of .05 was maintained for all statistical tests. Degrees of freedom were $(1,24)$ unless stated otherwise.

\section{Spatial attention}

Step 1: Spatial orienting in directionally cued trials. In the first step we examined spatial orienting in the cued trials. Table 2.1 displays response times as a function of the relevant task variables. A repeated measures 3 (cue: left, neutral, right) $\times 2$ (target: left, right) $\times 2$ (spatial frequency: high, low) ANOVA (Pillai's Trace) showed an interaction between cue and target, $F(2,23)=24.5, p<.001$. To examine costs and benefits, the same data were entered in a repeated measures 3 (validity: valid, neutral, invalid) $\times 2$ (target: left, right) $\times 2$ (spatial frequency: high, low) ANOVA. Significant costs (invalid versus neutral), $F=24.5, p<.001$, and benefits (valid versus neutral), $F=58.0, p<.001$, were present. From this it was concluded that arrow cues were successful in driving spatial attention. Responses were slightly faster to targets to the right $(271 \mathrm{~ms})$ than to targets to the left $(276 \mathrm{~ms}), F=3.6, p=.07$.This might be due to the fact that all responses were right-handed. In addition, responses were faster to low $(270 \mathrm{~ms})$ than to high $(277 \mathrm{~ms})$ spatial frequency targets, $F=12.7, p<.01$. Since spatial frequency did not interact with any of the other factors, it will not be included as a factor in the following steps of the analysis.

Table 2.1 Mean RT (ms) and standard error (indicated in parentheses) as a function of cue direction and target location, pooled across targets with high and low spatial frequency.

\begin{tabular}{lll}
\hline \hline & \multicolumn{2}{c}{ Target position } \\
\hline Cue direction & Left & Right \\
\cline { 2 - 3 } Left & $252(7)$ & $299(9)$ \\
Neutral & $276(9)$ & $266(8)$ \\
Right & $299(11)$ & $249(7)$ \\
\hline \hline
\end{tabular}


Directional orienting effects on EEG were studied in order to select the predictor variables used in step 2 and 3. Figure 2.1a presents the ERP results for every cue direction at a frontal and posterior electrode pair. ERP lateralisations that are sensitive to the direction of attention can be demonstrated most clearly by difference waves. Difference waves were computed for right and left-hemisphere electrodes, by first subtracting the ERP signal for shifts to the right from the ERP signal for shifts to the left. Then, left-hemisphere difference waves were subtracted from corresponding righthemisphere difference waves. Figure 2.2 shows double-subtracted ERPs. As a result of these double subtractions, a contralateral negativity is reflected by negative amplitude values and a contralateral positivity is reflected by positive amplitude values. ANOVAs on the consecutive $50 \mathrm{~ms}$ windows showed that localized effects of shifting spatial attention started at $150 \mathrm{~ms}$ and persisted until the end of the cue-target interval. Results will be described separately for posterior, central and anterior regions, and will only be described for the different electrodes in a region when lateralised effects are found to be affected by electrode (as indicated by interactions between cue direction, hemisphere and electrode). At posterior sites, there was an early positive component between 150 and $250 \mathrm{~ms}(7.3<F<17.8)$. This early positive component was followed by a negative component reflecting an early directing attention negativity, between 300 and $450 \mathrm{~ms}(6.4<F<20.7)$. A significant interaction between cue, hemisphere, and electrode was found in the same interval $(5.4<F(4,96)<8.3)$, caused by significant effects between 300 and $450 \mathrm{~ms}$ at P9/10 (4.4<F<10.4), P7/8 $(8.2<F<16.5)$ and P3/4 $(8.4<F<33.2)$ and significant effects only between 300 and $400 \mathrm{~ms}$ at PO7/8 $(12.0<F<15.4)$ and $01 / 2(9.7<F<15.0)$. Finally, at posterior sites, a positive component reflecting a late directing attention positivity was visible between 550 and $1200 \mathrm{~ms}(10.3<F<28.9)$. At central sites, there was a negative component reflecting an anterior directing attention negativity between 250 and $550 \mathrm{~ms}$ at C3/4 $(6.0<F<$ 65.3). At frontal sites there was a negative component reflecting an anterior directing attention negativity between 300 and $550 \mathrm{~ms}(5.5<F<29.6)$. A significant interaction between cue, hemisphere, and electrode was found between 300 and $450 \mathrm{~ms}(4.4<F$ $(2,48)<8.7)$, caused by stronger effects at FC5/6 $(25.7<F<37.3)$ than at F7/8 $(15.5$ $<F<19.9)$ and at F3/4 (11.4 $<F<18.6)$. Finally, frontal analyses showed another late negative component between 1000 and $1150 \mathrm{~ms}(4.6<F<8.2)$.

In sum, the interaction between cue direction and hemisphere for the different regions reflected three main components. First, an early positive component, between 150 and 250 ms after cue onset, maximal at posterior sites. Second, a negative component, between 250 and $500 \mathrm{~ms}$, reflecting an early directing attention negativity at posterior, and an anterior directing attention negativity at central and anterior sites. Third, a positive component, reflecting the late directing attention positivity, between 550 and $1200 \mathrm{~ms}$ at posterior sites.

For frequency band data, only absolute amplitude changes in the lower alpha 1 frequency band $(6-8 \mathrm{~Hz})$ were sensitive to spatial attentional orienting (similar to Babiloni et al. 2004 and Klimesch, Doppelmayr, Russegger, Pachinger, \& Schwaiger, 1998). No significant effects were found in higher frequency bands. Figure $2.1 \mathrm{~b}$ displays changes in the lower alpha1 band amplitude for one selected pair of electrodes. A decrease is indicated by a negative sign, an increase by a positive sign. A larger decrease of alpha amplitude was expected at the left hemisphere for cues to the right and at the right 
FC5

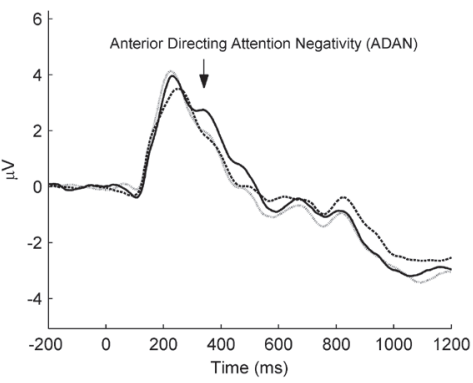

P7

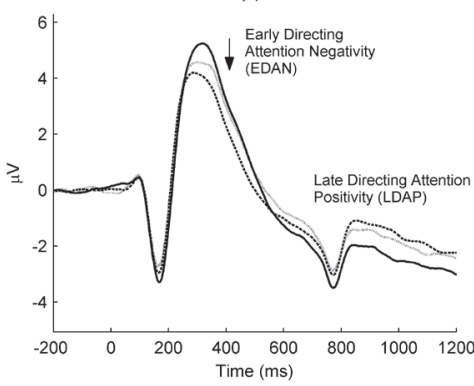

b

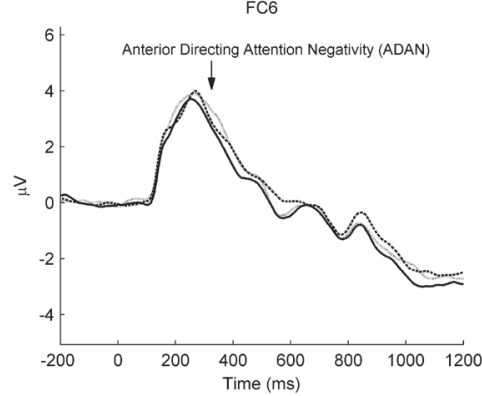

P8

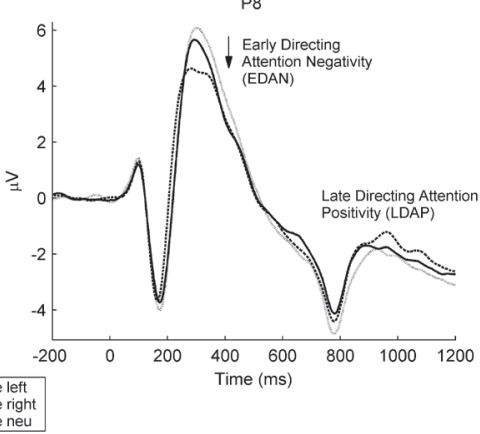

P7

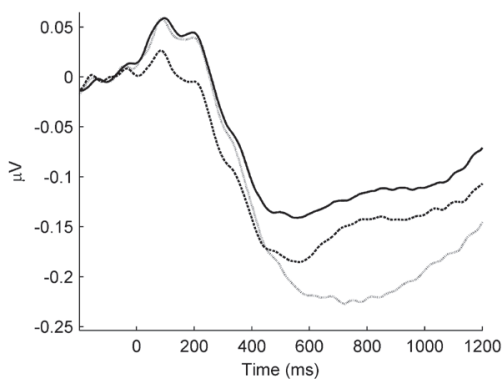

P8

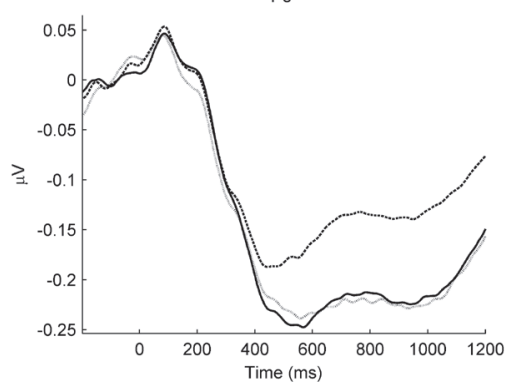

Figure 2.1 For the three cue conditions (a) grand-averaged ERPs at two selected electrode pairs, in the interval between cue onset and onset of the target. Three components contralateral to the direction of the cued attention shift are indicated: a frontocentral negativity (anterior directing attention negativity; ADAN), a posterior negativity (early directing attention negativity; EDAN), and a posterior positivity (late directing attention positivity; LDAP); (b) grand-averaged absolute changes of lower alphal amplitude at a selected posterior electrode pair, in the interval between cue onset and onset of the target. A decrease of lower alphal is reflected by a negative sign, an increase is reflected by a positive sign. 
hemisphere for cues to the left. Similar to ERP lateralisations, frequency band lateralisations that are sensitive to the direction of attention can be demonstrated most clearly by difference waves. Difference waves were computed, separately for right and lefthemisphere electrodes, by first subtracting the frequency band signal for shifts to the left from the frequency band signal for shifts to the right. Then, right-hemisphere difference waves were subtracted from corresponding left-hemisphere difference waves. Figure 2.3 displays these interactions between cue and hemisphere for all homologue electrode pairs. As a result of these double subtractions, a contralateral decrease is reflected by negative amplitude values and a contralateral increase is reflected by positive amplitude values. Significant interactions started at the beginning of the cue-target interval and persisted throughout this interval. At posterior areas, a significant decrease was present between 400 and $1200 \mathrm{~ms}(4.2<F<10.8)$. At central areas a decrease was present between 650 and $800 \mathrm{~ms}(4.5<F<5.2)$. Finally, at frontal areas, an early interaction between cue, hemisphere and electrode $(3.5<F(2,48)<4.2)$ between 0 and 150 ms was caused by a significant decrease at F7/8 $(4.5<F<4.8)$. It is remarkable that changes in the lower alpha 1 band were already significant at cue onset. The explanation probably lies in the temporal 'smearing' that is an inevitable consequence
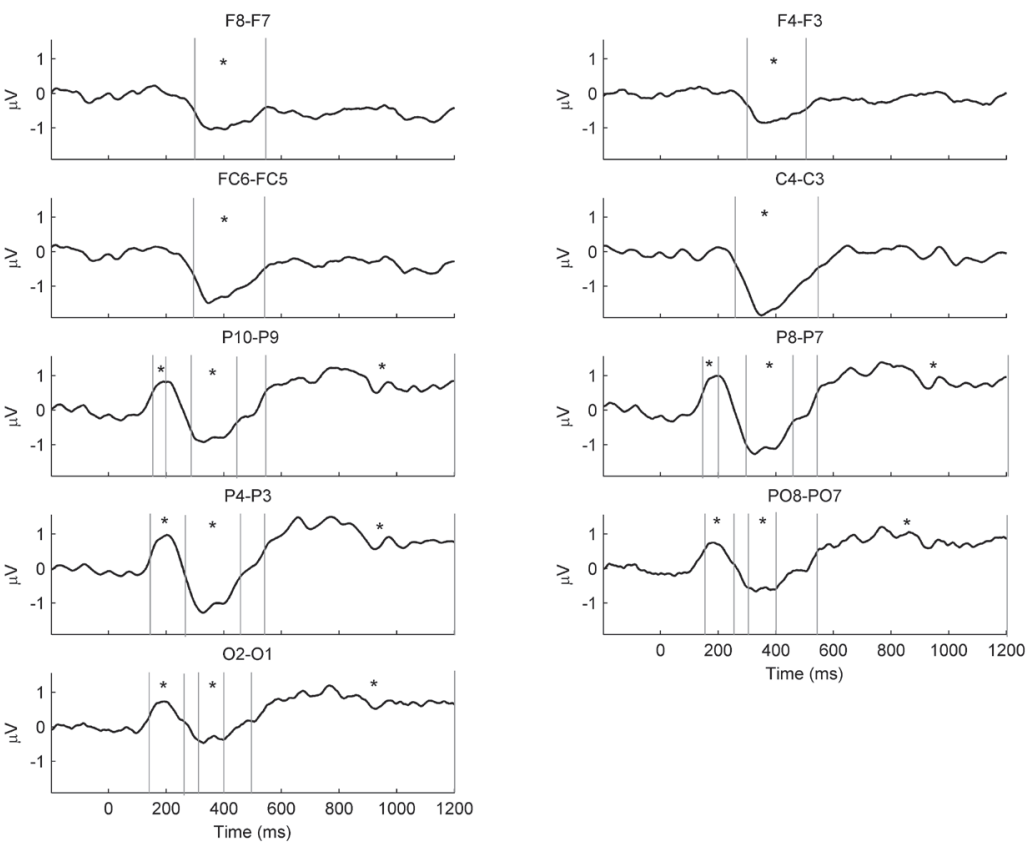

Figure 2.2 Grand-averaged ERP difference waves for all homologue electrode pairs in the interval between cue onset and onset of the target. Difference waveforms were generated by first subtracting ERPs in response to cues to the right from ERPs in response to cues to the left, and then subtracting the difference waves at left electrodes from the difference waves obtained for the corresponding right-hemisphere electrodes. Negative components contralateral to the direction of attention shifts are reflected by negative values, positive components are reflected by positive values. Significant time periods are indicated between vertical lines and marked with an asterisk. 

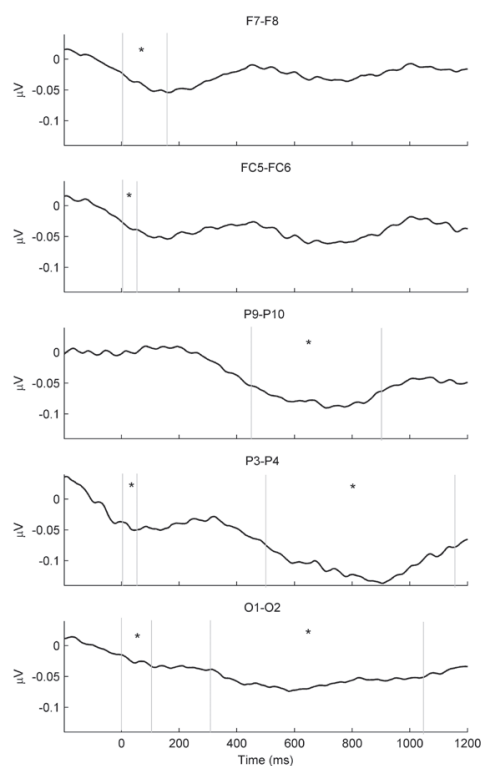
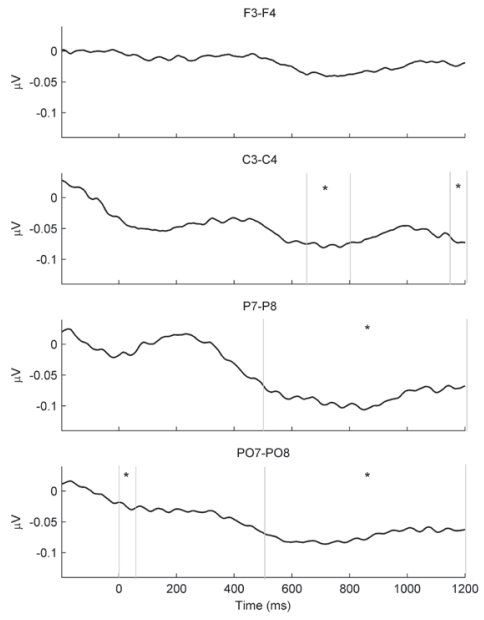

Figure 2.3 Grand-averaged lower alphal difference waves for all homologue electrode pairs in the interval between cue onset and onset of the target. Difference waveforms were generated by first subtracting changes in the lower alphal band in response to cues to the left from changes in response to cues to the right, and then subtracting the difference waves at right electrodes from the difference waveform obtained for the corresponding left-hemisphere electrode. Decreases of lower alphal contralateral to the direction of attention shifts are reflected by a negative sign, increases are reflected by a positive sign. Significant time periods are indicated between vertical lines and marked with an asterisk.

of the frequency estimation involved in computation of event-related changes in the frequency bands.

In sum, significant decreases of lower alpha1 contralateral to cue direction were present mainly at posterior areas, at a late (400-1200 ms) interval. Furthermore decreases were present early $(0-150)$ at frontal areas and late $(650-800 \mathrm{~ms})$ at central areas.

\section{Step 2: Cross-validation: predicting direction of attention in cued}

trials. ERP and frequency band lateralizations derived from the first step were used to predict cue direction in a single trial analysis for each subject. First, a cross-validation procedure was followed where half of all cued trials served to generate regression weights that were used subsequently to 'predict' cue direction in the second half of the cued trials.

In the ERP analysis, for each hemisphere, regional averages for electrodes at posterior, anterior, and central sites were computed in order to reduce the number of predictor variables in the logistic regression analysis and correlations between them. Right and left hemisphere values were subtracted and based on the results of step 1, 5 lateralized components were selected as predictor variables: one central component (250-550 $\mathrm{ms})$, an anterior component (300-500 ms), and three posterior components (150-250 
ms, 300-450 ms, and 550-1200 ms). To check for high collinearity between the predictor variables, variance inflation factors (VIFs) were computed for every subject ${ }^{3}$ (Kleinbaum, Kupper, Muller, \& Nizam, 1998). These never exceeded 5 and were therefore acceptable. Logistic regression was performed on the odd cued trials and regression weights were used subsequently to predict cue direction from ERP variables in the even cued trials. A 2 (predicted cue direction: left, right) $\times 2$ (target: left, right) ANOVA was carried out on the RTs, to test if the classifications were successful. For comparison, an additional 2 (actual cue direction: left, right) $\times 2$ (target: left, right) ANOVA was performed on these trials. There was an interaction between actual cue direction and target position, $F=$ $52.4, \mathrm{p}<.001$, and between predicted cue direction and target position, $F=5.5, \mathrm{p}<$ .05. No other effects were significant. In Table 2.2, mean RTs based on actual and predicted cue direction are shown. It was concluded that noise levels in the ERP during the cue-target interval were not too high for successful categorization of single trials into 'predicted cue direction left' and 'predicted cue direction right' trials, and that there should be sufficient power to successfully classify the neutral trials in a similar way, at least if attention is moved (randomly or systematically) in these trials.

In the analysis of event-related changes in the lower alpha1 band, 3 predictor variables were selected, again derived from the first step: one anterior component at F7/8 (0-150 ms), one central component (650-800 ms) and a posterior component (400-1200 $\mathrm{ms})$. VIFs never exceeded 2 and were therefore acceptable. In all other respects, the cross-validation was the same as for the ERPs. The relevant interaction between predicted cue direction and target position was not significant, $F<1$. Thus, for the lower alpha1 band the cross-validation failed and therefore, only ERP predictor variables were used in the third step.

Step 3: Predicting direction of attention in neutral trials. The same ERP predictor variables that were used in the second step were now used in a logistic regression analysis with all cued trials, and their regression weights were subsequently used to predict the direction of attention in the neutral trials. After this classification, the neutral trials were sorted into 'predicted direction attention left' and 'predicted direction attention right' trials, and this classification was combined with the actual target position in an analysis of RTs. Mean RTs were submitted to a 2 (predicted direction attention: left, right) $\times 2$ (target: left, right) ANOVA. The results are presented in the last part of Table 2.2. There was no interaction between the predicted direction of attention and target position (interaction contrast $0.25 \mathrm{~ms}, F<1$ ).

To further increase power, the analyses were repeated in a subset of subjects for whom the classification of trials into 'cue left' and 'cue right' in step 2 (the crossvalidation) was above chance level as determined by binomial tests. The percentage of correctly classified trials significantly exceeded chance level $(50 \%)$ in thirteen subjects (ranging from $63 \%$ to $76 \%$, the rest ranging from $42 \%$ to $62 \%$ ). The 2 (predicted direction of attention: left, right) $\times 2$ (target: left, right) ANOVA on neutral trials again showed

\footnotetext{
3 The variance inflation factor (VIF) $=1 / 1-R^{2}$ (Kleinbaum et al., 1998), is often used to measure collinearity between predictor variables in regression analyses. Scores lower than 10 are acceptable. In the current analysis, the average of electrodes was used to compute anterior, posterior and central components. This was done, because individual electrodes correlated very high with each other, leading to VIFs $>10$.
} 
no interaction between the predicted direction of attention and target position (interaction contrast $0.49 \mathrm{~ms}, F(1,12)<1)$. For frequency band results, the percentage of correctly classified trials exceeded chance level (50\%) in only two subjects (both $62 \%$, the rest ranging from $37 \%$ to $57 \%$ ).

Table 2.2 Mean RT (ms) and standard error (indicated in parentheses) for target left and target right as a function of cue direction for cued trials with an even trial number, predicted cue direction for cued trials with an even trial number (cross-validation), and predicted direction of attention for all neutral trials. The validity effect (difference between valid and invalid cues) is also reported for the different conditions.

\begin{tabular}{lll}
\hline \hline & \multicolumn{2}{c}{ Target Position } \\
\cline { 2 - 3 } & & Left \\
\hline Actual cue direction \\
Left & $253(7)$ & $300(9)$ \\
Right & $297(11)$ & $249(7)$ \\
Invalid - Valid & 44 & 51 \\
Predicted cue direction & & \\
Left & $258(7)$ & $262(7)$ \\
Right & $268(9)$ & $258(8)$ \\
Invalid - Valid & 10 & 4 \\
Predicted direction of attention & & $266(8)$ \\
Left & $277(10)$ & $265(8)$ \\
Right & $276(9)$ & 1 \\
Invalid - Valid & -1 & \\
\hline \hline
\end{tabular}

Originally, the logistic regression expressed the predicted direction of attention as an estimated probability that the trial was an 'attention right' trial. This was dichotomized later . A paired comparison of the average absolute values of the logodds ${ }^{4}$ of all neutral trials on the one hand and the even cued trials on the other hand showed significantly smaller logodds value for the neutral trials (.63) than for the cued trials (.77), $t$ $(24)=2.9, p<.01$. This confirms our conclusion that classification in neutral trials was largely random, as expected when attention does not move in these trials.

In sum, cross-validation results showed that ERP components could be used successfully in cued trials to determine the direction of attention in single trials. For neutral trials, evaluation of the predicted direction of attention using RTs and logodds values revealed no evidence of spatial attentional orienting in the cue-target interval of these trials.

4 Log-odds $=\ln [p /(1-p)]$ (Howell, 2002). For the current data, negative log-odds values indicate shifts to the left, while positive values indicate shifts to the right. 


\section{Nonspatial attention}

To analyse nonspatial attention, nonlateralized preparatory EEG activity was analysed. For this, neutral trials were classified into three equal-sized RT bins for every target position. ${ }^{5}$ Both the nonlateralized $\mathrm{CNV}$ component and nonlateralized changes in the alpha band $(8-14 \mathrm{~Hz})$ were examined at $\mathrm{C} 3, \mathrm{C} 4, \mathrm{Cz}$ and Pz. Successive mean voltage values every $50 \mathrm{~ms}$ in the cue-target interval, from $400 \mathrm{~ms}$ before target onset until target onset, served as dependent variables. A comparison of the fastest and the slowest bin was made. The ERP at $\mathrm{Cz}$ is shown in Figure 2.4a. As onset of the target stimulus neared, at all four locations a CNV component developed that was more negative for faster responses and that continued until target presentation. One-way within subjects ANOVAs of slow versus fast RTs showed that this effect was significant between 800 and $1200 \mathrm{~ms}$ at $\mathrm{C} 3(7.2<F<38.2)$, at $\mathrm{C} 4(8.8<F<30.4)$, and at $\mathrm{Cz}(16.2<F<44.8)$ and between 1000 and $1200 \mathrm{~ms}$ at $\mathrm{Pz}(8.0<F<11.2)$. These results are consistent with an interpretation of the $\mathrm{CNV}$ as a reflection of preparatory activation preceding an imperative stimulus (Brunia \& van Boxtel, 2001), and comparable to the results reported by Hackley and Miller (1995) where the relation with RT was also shown.

Insert Figure 4

Figure 2.4b displays the results of the same analysis on nonlateralized changes in the alpha band. As expected, a larger decrease in alpha amplitude preceded target onset for fast than for the slow responses at all four locations. This effect was significant between 850 and $1150 \mathrm{~ms}$ at C3 $(4.6<F<8.3)$ (and marginally significant $(p<.01)$ between 1150 and $1200 \mathrm{~ms}(F=3.1)$, between 800 and $1000 \mathrm{~ms}$ at $\mathrm{C} 4(7.4<F<13.8)$, between 850 and $1200 \mathrm{~ms}$ at $\mathrm{Cz}(4.7<F<9.0)$ and between 900 and $1050 \mathrm{~ms}$ at $\mathrm{Pz}$ $(4.9<F<5.3)$ (and marginally significant $(p<.01)$ between 1050 and $1200 \mathrm{~ms}$ (3.1 $<F<4.3)$.

To summarize, these results show that variations in the speed of responding to targets that follow neutral cues covary with two measures that can be taken as indices of nonlateralized preparatory activity or nonspatial attention to the target.

\section{Additional ERP analyses}

Regarding the lateralized ERP components related to spatial attention that were described in the first step, we performed some extra analyses that are relevant to the recent discussion about the processes they reflect (Nobre et al., 2000; Van Velzen \& Eimer, 2003). It was reasoned that if subjects who showed larger effects on a component in the ERP in the cue-target interval also showed larger attention effects on RTs to the target, this would support the idea that this component reflects attentional orienting. Conversely, if a component reflects asymmetries unrelated to attention, e.g. purely sensory lateralizations related to small asymmetries in the shape of the cue (Nobre et al., 2000; Van Velzen \& Eimer, 2003), it should not correlate with the overt behavioral manifestation of spatial attention. For ERP data, the averaged successive $50 \mathrm{~ms}$

\footnotetext{
5 Bins were computed separately for every target position but not for every spatial frequency of the target stimulus. This was done because in the RT analysis with only neutral trials, a 2 (target: left, right) $\times 2$ (spatial frequency: high, low) ANOVA showed an effect of target position, $F(1,24)=11.1, p<.05$, but not spatial frequency, $F(1,24)=2.8, p$ $=.11$.
} 
windows corresponding to the early positivity, the early directing attention negativity, the anterior directing attention negativity, and the late directing attention positivity at various electrode sites were correlated, between subjects, with the attention effect on RT, computed as mean RT(invalid) - mean RT(valid). Although none of the correlations was significant (tested one-sided) in this first analysis, noticeably all correlations at posterior, frontal, and central areas between the early directing attention negativity and anterior directing attention negativity and the RT effect were negative as expected if these components reflect attentional orienting. In addition, a marginally significant correlation was found between the anterior directing attention negativity and the RT effect at F3/4: $r(25)=-.33, p=.055$. Inspection of the scatter plots suggested that one subject's data were outliers in the analysis of the positive component at P7/8 and $\mathrm{P} 9 / 10$ and in the analysis of the early directing attention negativity at P7/8. This was confirmed by a comparison analysis of Cook's distances with the maximum value of 25 subjects (0.75; Kleinbaum et al., 1998). Correlations, recomputed without this case were significant for the early directing attention negativity at posterior sites (P9/10: $r$ $(24)=-.34, p=.05$; P7/8: $r(24)=-.40, p<.05)$. It was also striking that the other strong cue $\times$ hemisphere effects on ERPs did not show these correlations (early positivity: max. $r(24)=-.11$; late directing attention positivity: $\max . r(24)=.14$ ) and also varied in correlation sign across electrodes.

a

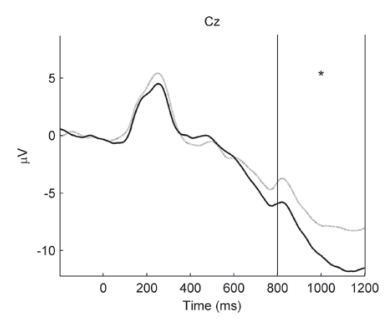

b

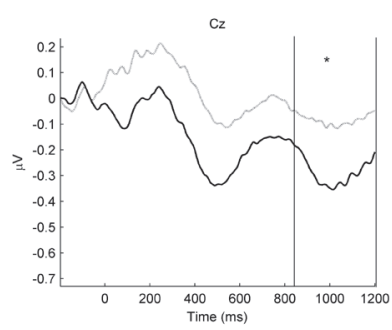

Figure 2.4 (a) Grand-averaged ERP waveforms and (b) absolute changes (decrease is negative sign, increase is positive) in the alpha $(8-14 \mathrm{~Hz})$ band at $\mathrm{Cz}$, in the interval between cue onset and onset of the target, as a function of response speed (slow or fast). Significant time periods are indicated between vertical lines and marked with an asterisk.

\section{Discussion}

Nonspatial attention and spatial attention in neutral trials were examined in an analysis of EEG and RTs. It was tested whether variance in RTs of neutral trials was related to variance in nonspatial attention and to random or systematic switches of spatial attention towards the location where a target could appear.

Spatial attention. The analyses of spatial selective attention were divided into three steps. First, attentional processing in cued trials was examined. Its effects on the ERP during the cue-target interval were mostly similar to the reports in the literature, starting with work of Harter et al. (1989). There was a negativity contralateral to the cue direction between 250 and $500 \mathrm{~ms}$ after the cue, reflecting the early directing attention negativity at posterior electrodes and the anterior directing attention negativ- 
ity at anterior and central electrodes, and a contralateral positivity between 500 and $1200 \mathrm{~ms}$, reflecting the late directing attention positivity, at posterior electrodes. One effect, however, has not been reported in other studies. This was an early posterior contralateral positivity, between 150 and $250 \mathrm{~ms}$. One possibility is that it is related to sensory processing of the slightly asymmetric cue stimuli, but in principle, it could also reflect a fast orienting reaction.

Event-related changes were also analysed for a number of EEG frequency bands. Clear lateralized changes were present contralateral to the cue direction, in the lower alpha1 band (6-8 Hz), mainly at posterior areas, at an early (0-250 ms) and a late (500$1200 \mathrm{~ms}$ ) interval. Worden et al. (2000) reported an increase of alpha (8-14 Hz) contralateral to those stimuli that had to be ignored during a spatial cueing paradigm. In a recent review (Ward, 2003) it was concluded that increases of 8-14 Hz alpha indicate active suppression of activity related to distractors instead of "cortical idling" (Pfurtscheller, 1992). Thus, the absence of changes in the 8-14 Hz band in our results may be related to the fact that in our detection task subjects had to detect the target in any case, whereas in Worden et al. subjects only had to respond if the target appeared at the cued location, thus promoting active suppression of the uncued location. Furthermore, the discrimination task used by Worden et al. may require more attention because its perceptual load is higher (Lavie, 1995).

Lately, there has been more discussion about the relation between changes in the alpha band and attentional processing. Although the alpha band is traditionally defined as ranging from about 8 to $14 \mathrm{~Hz}$, it was proposed that it should be divided into a number of bands since they reflect quite disparate cognitive processes (Klimesch et al., 1998). It was shown that mainly slower alpha frequencies (6.5-10.5) reflect attentional processes. This was supported by a recent cueing study (Babiloni et al., 2004) that reported modulation by temporal orienting only in the lower alpha band $(6-10 \mathrm{~Hz})$. Our results extend the latter result to spatial orienting.

In summary, in the first step, both behavioral data and lateralizations in the ERP and EEG frequency band results during the cue-target interval showed effects of spatial orienting that were predicted or were easy to reconcile with the extant literature.

In the second step, EEG lateralizations derived from the first step were used to predict cue direction in a single trial, cross-validation, logistic regression analysis on ERP and frequency band lateralizations for each subject. Regression weights were determined in an analysis of the odd-numbered trials and subsequently used to predict cue direction in the even trials. The success of this prediction was demonstrated for ERP (but not frequency band) variables by a significant interaction between 'predicted cue direction' and 'target position' on RT. The RT effect was smaller than in the first step (as shown by table 2.2), probably because sorting the trials on the basis of these predictor variables involves some noise. Nevertheless, and importantly, the results showed that it was possible to predict the direction of attention in single trials at better than chance level by using only the lateralized ERP during the cue-target interval. From this it was concluded that there should be sufficient power to predict the direction of attention in a similar way in neutral trials if similar shifts of attention are present in these trials.

In the third step, ERP-lateralizations were used to predict the direction of attention in neutral trials. Time periods, electrode positions and regression weights of these lateralizations were similar to those that were used in the second step for directionally 
cued trials. The classification was evaluated again by RT results. It was reasoned that spatial attention effects on RT should be obtained in this step if (a) classification was sufficiently reliable, and (b) attentional shifts leading to RT effects actually occurred. The evidence for reliability had been obtained in step 2. However, predicted direction of attention and target position effects on RT in neutral trials did not interact. Furthermore, a comparison of estimated probabilities of the prediction of the direction of attention for cued and neutral trials showed that for neutral trials the prediction was closer to chance. Together, these findings show that although single trial ERP lateralizations could be used to classify cued trials in a reliable way, demonstrating sufficient power of the approach, no similar classification of neutral trials was possible. Therefore, it is unlikely that switches of attention were made in the neutral trials that were comparable to those in the cued trials. Instead, neutral trials might be characterized by a diffuse state of attention (de Gonzaga et al., 1987) or attention might remain at fixation when a neutral cue is presented (Posner, 1978).

It should be noted that regression weights for ERP-variables and the selection of the variables (time periods and locations) that were applied in the neutral trials were derived from the cued trials. It cannot be excluded that some ERP-variables derived from the cued trials might merely reflect the extraction of spatial information from the cue, not attentional orienting itself. In that case, these variables might be successful in sorting the cued trials, but not in neutral trials, since neutral cues do not contain specific spatial information. Secondly, this method assumes that the shifts of attention in neutral trials are comparable to shifts of attention in cued trials. Some authors, however, have proposed that the time to process cues might differ between directional and neutral cues (Jonides \& Mack, 1984). In addition, different brain areas might be responsible for shifts of attention in cued and neutral trials. Obviously, if these differences between orienting in response to directional and neutral cues are real, the present approach could overlook attentional orienting in neutral trials. In this regard, the firm conclusion we may draw on the topic of spatial orienting in neutral trials is that if it happens at all, it is not similar, in timing and brain areas involved, to attentional orienting in cued trials.

Nonspatial attention. In addition to spatial attention, nonspatial attention in neutral trials was studied. An increase in anticipatory attention in neutral trials, reflected by a decrease in RT, was related to a larger nonlateralized CNV component preceding target onset and a larger decrease of amplitude in the alpha band. A similar relation between the CNV amplitude and RT was demonstrated by Hackley and Miller (1995) and decreases in the alpha band have repeatedly been related to increases in attention and activation (e.g. Boiten, Sergeant, \& Geuze, 1992; Dujardin et al., 1993; Vazquez Marrufo, Vaquero, Cardoso, \& Gómez, 2001) and stimulus anticipation (Bastiaansen \& Brunia, 2001; Filipovic, Jahanshahi, \& Rothwell, 2001; Bastiaansen, Böcker, \& Brunia, 2002). As argued by Brunia and Damen (1988), in a warned reaction time experiment, motor preparation and preparation for the stimulus are confounded. Miniussi, Wilding, Coull and Nobre (1999) proposed that the improvement in RT after a warning signal is due to an internal time estimation process that starts when a warning signal is given. Coull and Nobre (1998) used a temporal analog of a spatial orienting task and demonstrated that comparable to information about location, information about time intervals can be used dynamically to direct attention and improve behavioral performance (for a review, 
see Nobre, 2001).With the current data it is not possible to discriminate processes of preparation for the stimulus, motor response and time estimation processes. Still, our results suggest that during neutral trials at least subjects do not passively wait until the target is presented, but that some variations in preparation for the target or response explain variations in RT.

Interpretation ERP components: additional analyses. Although both the early directing attention negativity and the late directing attention positivity have been related to attentional orienting, there is no agreement on a more specific interpretation of these components. Harter et al. (1989) proposed that the early directing attention negativity is related to interpretation of the cue and directing of attention, and that the late directing attention positivity is related to target preparation. In addition it was proposed that the anterior directing attention negativity, because of its location, is associated with the control of directing attention (Hopf \& Mangun, 2000; Nobre et al., 2000).

In a recent discussion the functional significance of the early directing attention negativity has been questioned (Nobre et al., 2000; Van Velzen \& Eimer, 2003). Van Velzen and Eimer found this component to be independent of attention directing properties of cues. Instead, it was determined by the position of the relevant side of a cue. Therefore, it was concluded that instead of attentional control, the component reflects selection of task-relevant aspects of cue stimuli. However, the present results provided independent evidence that it is related to attentional control. In a between-subject correlation analysis, it was shown that the posterior early directing attention negativity was related to the attention effect on RT to the target. Although our cue stimuli were slightly asymmetric and could thus, in principle, lead to lateralized sensory components, these components would not be expected to be modulated by later behavioural manifestations of attentional orienting.

Conclusions. In conclusion, to study spatial shifts of attention in neutral trials, we have developed a new method. Single trial ERP lateralizations were successfully used as predictor variables to classify single trials with informative cues in a reliable way, demonstrating sufficient power of the method. Application of the method to neutral trials lead to the conclusion that attention is not shifted in these trials in a way that is comparable to shifts of attention in cued trials. This argues against the idea that RT variations in neutral trials can be explained by random or systematic switches of attention in these trials. However, an analysis of nonlateralized EEG activity (the 8-14 Hz alpha band and the CNV component) in the cue-target interval showed that variations in the RTs of neutral trials are not merely due to meaningless noise but are indeed related to variations in attention. These effects on the alpha frequency band and ERPs can be interpreted as manifestations of variations in nonspatial attention. 


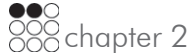




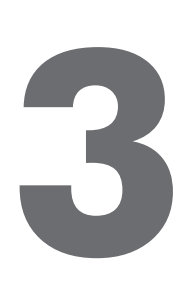

\title{
Lateralized ERP compo- nents related to attentional orienting in an all-go and a go-nogo task:
}

\author{
Discriminating shifts of attention \\ from sensory aspects of the cue
}

In two spatial cueing experiments the functional significance of lateralized ERP components after cue-onset was examined. A simple detection task was used in experiment 1, whereas responses were only required to valid targets in experiment 2 . The main goal was to discriminate components related to sensory aspects of the cue and components related to the direction of attention. Therefore, cue shape and task instruction were controlled in two tasks. Arrows were used in one task and physical properties were balanced across attention-left and attention-right conditions. Nonlateralized visual cues were used in the other task. Lateralized cue effects and modulation after stimulus-onset were stronger in experiment 2 . An early posterior component, but not a later posterior negativity (EDAN), was related to the physical shape of arrows. An anterior negativity (ADAN) and a posterior positivity (LDAP) were related to the direction of attention. Finally, the EDAN appeared to be specific for conditions with arrow cues and thus may be related to the encoding of directional information.

Jongen, E.M.M., Van der Heiden, J.S.H., \& Smulders, F.T.Y. (2007). Lateralized ERP components related to attentional orienting in an all-go and a go-nogo task: Discriminating shifts of attention from sensory aspects of the cue. Manuscript submitted for publication. 
Spatial attention can modulate information processing by improving accuracy and speeding up detection and discrimination of stimuli (e.g. Downing, 1988; Posner \& Snyder, 1975). One way to study spatial attention is with a spatial cueing paradigm in which a cue predicts the likely position of a target stimulus. Reaction times (RTs) to stimuli that appear at the cued location (valid trials) are faster and responses are more accurate than to stimuli that appear at an uncued location (invalid trials) (e.g. Posner, Nissen, \& Ogden, 1978). In a large number of spatial cueing studies event-related brain potentials (ERPs) have been measured to more closely study the time course of attention and isolate components related to (effects of) attentional orienting. At first, comparable to RT research, attentional orienting was studied by examining modulatory effects of attention on the target stimulus. A sequence of ERP components is evoked over lateral occipital sites consisting of an early P1 component (onset at about 70$90 \mathrm{~ms}$ ), followed by an $\mathrm{N} 1$ component (onset at about 100-120 ms) (for reviews, see Anllo-Vento, Schoenfeld, \& Hillyard, 2004; Luck, Woodman, \& Vogel, 2000). For validly cued stimuli, in comparison to invalidly cued stimuli, amplitude enhancements for both components are usually found (e.g. Mangun, Hillyard, \& Luck 1993) although in some cases, despite behavioral effects, only the P1 (Mangun \& Hillyard, 1991), the N1 (Eimer, 1994), or neither of these components (Eimer, 1993) were modulated. In an increasing number of studies ERPs have now been used as measures of the brain's response to the cue instead of the target stimulus. A sequence of lateralized components with a positive or negative voltage over the hemisphere contralateral to the direction of attention has repeatedly been reported. These components are suggested to reflect different stages in the control of attention (e.g. Eimer \& Van Velzen, 2002; Harter, Miller, Price, LaLonde, \& Keyes, 1989; Hopf \& Mangun, 2000; Nobre, Sebestyen, \& Miniussi, 2000). The sequence consists of an early directing attention negativity (EDAN) at posterior electrodes between about 200 and $400 \mathrm{~ms}$ post cue, an anterior directing attention negativity (ADAN) at frontal and central sites between 300 and $500 \mathrm{~ms}$ post cue, and a late directing attention positivity (LDAP) at posterior sites that starts around $500 \mathrm{~ms}$ post cue. The EDAN has been hypothesized to reflect encoding of the meaning of a cue, and the resulting initiation of an attentional shift to the cued location (Harter et al., 1989; Hopf \& Mangun, 2000; but see Van Velzen \& Eimer, 2003). Originally, the LDAP has been assumed to reflect modulation of the cortical excitability in those regions that process the upcoming visual information (Harter et al., 1989). However, inconsistent with this interpretation, the LDAP decreased before target onset (Hopf \& Mangun, 2000), and when SOA was varied it was shown to be time-locked to cue onset instead of target onset (Van Velzen, Forster, \& Eimer, 2002). Furthermore, it should be noted that in a number of cueing studies no LDAP was found (Mangun, 1994; Nobre et al., 2000; Talsma et al., 2005; Yamaguchi, Tsuchiya, \& Kobayashi, 1994). In line with the common view that frontal cortical structures contribute to the attentional control of sensory processing (LaBerge, 1997, Posner et al., 1987; Posner \& Petersen, 1990), the ADAN has been associated with attentional control and redirecting attention in space. Recent studies have shown that the LDAP and the ADAN might be related to supramodal processes of attentional control, and thus not be limited to the visual modality (Eimer \& Van Velzen, 2002; Eimer, van Velzen, \& Driver, 2002; Van Velzen \& Eimer, 2003; but see, Green et al., 2005; Green \& McDonald, 2006). 
To study these lateralized components after cue onset, conditions in which attention is cued to the left are directly compared to conditions in which attention is cued to the right. Nobre, Sebestyen, and Miniussi (2000) recognized that this method confounds the features of the cue and the direction of attention. Instead of being a reflection of attentional orienting, ERP components that differ between left and right cue conditions might therefore reflect sensory aspects of the cue. They attempted to neutralize this confound in an elegant way by using an identical stimulus to cue attention to the left and to the right. A diamond-shaped stimulus with a green and a red side was used. In different blocks, participants were asked to orient their attention either to the direction indicated by the green or by the red side of the cue, thus balancing physical stimulus features across blocks. Although identical cues were now used to direct attention to the left and to the right, selection of a lateralized part of the cue was a prerequisite for every shift of attention and the locus of the selected side (left, right) was $100 \%$ confounded with the direction of attention. To address this confound, Van Velzen and Eimer (2003) added extra conditions to balance the position of the relevant part of the cue across attention-left and attention-right conditions. Whereas the ADAN and the LDAP were elicited contralateral to the direction of attention, irrespective of the position of the relevant part, at the latency of the EDAN a negativity was elicited contralateral to the position of the relevant part, irrespective of its direction. It was therefore concluded that whereas the ADAN and LDAP represent correlates of the direction of attention, the EDAN is unrelated to the direction of attention but similar to an N2pc component (Luck \& Hillyard, 1994; Eimer, 1996), reflecting the selection of taskrelevant aspects of cue stimuli.

Here, we present two alternative ways to measure attentional orienting independent of sensory aspects of the cues by controlling their physical shapes. In the first of two tasks, using standard arrow cues, the physical properties of arrows were balanced across attention-left and attention-right instructions. That is, in one instructed mapping, attention was to be oriented in the direction indicated by the arrow, whereas in the other instructed mapping, attention was to be oriented against the direction of the arrow. ERP components related to attentional orienting should covary with the instructed direction of orienting, but not the physical direction of the arrow, whereas the opposite holds for components related to the physical shape of the arrow.

The cues that were used by Van Velzen and Eimer (2003) represented ideal conditions to elicit an N2pc as they required selection of a lateralized part. If every EDAN would in fact be an N2pc, no EDAN should be elicited when cues do not have any lateralized aspects. Eimer, Van Velzen, Forster, and Driver (2003) already showed that the EDAN was absent when auditory cues directed attention in a cross-modal cueing study. Here, in the second (unimodal) cueing task, vertical line cues were used to direct attention to peripherally presented visual stimuli. The direction of attention was determined by the height of the lines. Thus it was ruled out that any (lateralized) sensory aspects of the cue lead to lateralized ERP components, leaving only the components that are truly related to the direction of attention.

In the first experiment, participants were asked to respond to validly cued stimuli as well as invalidly cued stimuli. In the second experiment, participants were asked to respond only to infrequent target stimuli on valid trials. 


\section{EXPERIMENT 1}

\section{Method}

\section{Participants}

Thirty volunteers participated in the study. All gave informed consent and received course-credits for participation. Six were excluded because of excessive blinks, eye movements, because data were noisy or because they did not follow the instruction of the spatial cueing task. Thus, 24 participants (age 18-38, mean age 22, 20 female) remained in the sample. Twenty-two participants were right-handed, and all had (corrected to) normal vision. The experimental methods had ethical approval from the institutional ethics committee.

\section{Behavioral tasks and procedure}

Participants were seated in a dimly lit, sound-attenuated room. Tasks were presented on a VGA monitor that was placed outside the room behind a window, at a viewing distance of $75 \mathrm{~cm}$. There were two tasks, one with arrow cues ("the arrow task") and one with vertical line cues that varied in height ("the line task"). In the arrow task, cues consisted of an arrow to the left $(<<)$, to the right $(>>)$ or a neutral, double arrow $(<>)$. In the "congruent mapping" the instructed direction of attention and the physical direction of the arrow cues corresponded ( $<<$ for left, $>>$ for right). In the "incongruent mapping" the instructed direction of attention was reversed $(<<$ for right, $>>$ for left). In the line task, cues consisted of vertical lines that were short (50 pixels, $1.8^{\circ}$ ), medium (100 pixels, $3.5^{\circ}$ ) or long (150 pixels, $\left.5.3^{\circ}\right)$. Line stimuli of medium length served as neutral cues. Short line stimuli served as cues to the left and long lines as cues to the right in one of the instructed mappings ("short-left mapping"), and this arbitrary allocation was reversed in the other instructed mapping ("short-right mapping"). To make cues easily discriminable from each other, lines of medium length were thinner (one pixel wide, $0.04^{\circ}$ ) than short and long line cues (4 pixel wide, $0.2^{\circ}$ ). To prevent interference from switching between instructed mappings, every subject participated in one of the arrow mappings and one of the line mappings. The mappings for arrow and line tasks were balanced across participants.

In both tasks, a dark gray-and-light gray, vertical square-wave grating stimulus $\left(2.1^{\circ}\right.$, 7.3 cycles/degree) was presented left or right $\left(6.8^{\circ}\right)$ from fixation, on a gray background with the same average luminance. The stimulus was therefore hard to discriminate from it's the background which served to increase attentional demands.

An example of a trial is shown in Figure 3.1. Each trial started with a fixation line (100 ms) that stayed on throughout the trial. A cue was presented (100 ms) and after a randomized short $(517 \mathrm{~ms})$ or long $(817 \mathrm{~ms})$ blank interval the peripheral stimulus (100 ms) was presented, followed again by an intertrial-interval (800-1000 ms). In one third of the trials, a neutral cue with a predictive validity of $50 \%$ was presented. In the remainder of the trials cues with a predictive validity of $80 \%$ for the left or the right stimulus position were presented equally often. Participants were instructed to move their attention to the location indicated by the cues. They were informed about the predictive validity of the cues and that moving their attention would decrease their RT. 
In addition they were instructed to maintain central eye fixation and to respond quickly and accurately to every peripheral stimulus, irrespective of its location by pressing a response button with their right index finger (all-go, simple detection task). Corrective feedback was given on misses and false alarms and on responses that preceded stimulus onset, were too fast $(<120 \mathrm{~ms})$, or too slow $(>800 \mathrm{~ms})$. Catch trials $(17 \%)$ were added to prevent premature responses.

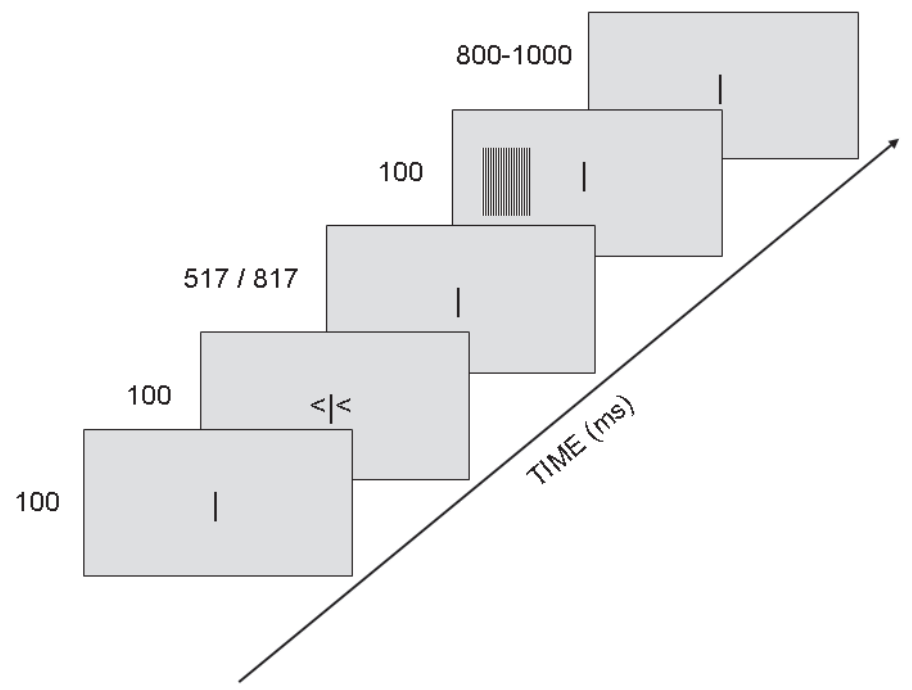

Figure 3.1 Schematic illustration of a trial from one of the arrow mappings. Stimuli are not to scale. The arrow points to the left and the stimulus is presented at the left position. For the congruent mapping this would be a valid trial, for the incongruent mapping it would be invalid. In the line task, instead of arrows vertical lines of short or long length were mapped to the left or right cued direction of attention and lines of medium length served as neutral cues. The indicated timing was used in the first experiment. In the second experiment slight adjustments were made to the timing and the stimuli, as described in the text.

Every task consisted of a 72-trial practice session and a 576-trial experimental block (preceded by 8 warm-up trials that were not included in the analyses). The proportion of valid : invalid : neutral : catch trials in the experimental block was $16: 4: 10: 6$. Participants received feedback about their reaction time after every 50 trials and could proceed with the task when they felt ready. After participants participated in one task, they were given a longer break.

After the preparations for the EEG recordings, participants performed on an eye movement calibration task to determine the voltage-level corresponding to an eye movement to the left and right stimulus locations. These values were measured for every individual and were used to determine individual trial removal criteria. Then, a blink calibration task was presented in which spontaneous blinks were promoted by demanding constant fixation to detect slow color changes of a fixation cross. The blink correction factor was derived from this task and was used for offline correction of trials with eyeblinks in the attention-tasks. After these calibration tasks the arrow and line task were presented. 


\section{Data recording}

Electroencephalographic (EEG) activity was recorded continuously, via NeuroScan 4.3, from 30 channels, using tin electrodes mounted on an elastic cap (Quik-Cap) and positioned according to the 10-20 System. The montage included 5 midline sites (Fz, FCz, $\mathrm{Cz}, \mathrm{Pz}, \mathrm{Oz}$ ) and 25 lateralized sites (FP1/FP2, F3/F4, F7/F8, FC5/FC6, C3/C4, CP5/CP6, T7/T8, P3/P4, P7/P8, P9/P10, PO7/PO8, 01/O2 and the right mastoid). ${ }^{1}$ The left mastoid (A1) was used as the reference for all electrodes and AFz functioned as the ground. Tin electrodes were also used to bipolarly record vertical (above and below the left eye) and horizontal (at outer canthi of both eyes) electro-oculograms (EOGs). Electrode impedance was kept below $5 \mathrm{k} \Omega$ during recording, amplifier bandpass was $0.05-100 \mathrm{~Hz}$, and digitisation rate was $500 \mathrm{~Hz}$.

The electrodes were re-referenced offline to the average of right and left mastoids. Trials with eyeblinks were corrected with a regression procedure (Semlitsch, Anderer, Schuster, \& Presslich, 1986), using the blink correction factor derived from the blink calibration task. Data of the HEOG channel was re-filtered with a $10 \mathrm{~Hz}(24 \mathrm{~dB} / \mathrm{oct}$.) low pass filter, data of all other channels were re-filtered with a low pass filter of $30 \mathrm{~Hz}$ (48 dB/oct.). Epochs were made starting $200 \mathrm{~ms}$ before cue onset and ending $300 \mathrm{~ms}$ after onset of the peripheral stimulus. Incorrect response trials, trials with a voltage exceeding $+/-100 \mu \mathrm{V}$, and trials with eye movements were excluded from the analyses. To exclude trials with eye movements, for each individual $30 \%$ of the median voltage for an eye movement to the left and to the right stimulus position, taken from the data of the eye calibration task, served as a criterion in the orienting tasks, starting from cue-onset. Subsequently, individual averages for cues to the left and cues to the right were computed and their HEOG channel was inspected for systematic deviations. Peak detection was done in the entire cue-target interval, and if residual, systematic, horizontal activity ( $>5 \%$ of the criterion) was present, participants were excluded from the analyses. This led to the exclusion of one subject, and the residual average percentage of HEOG activity in all participants included in the analyses was $1.6 \%$ for the arrow task (left $1.4 \%$, right $1.8 \%$ ) and $1.2 \%$ for the line task (left $1.4 \%$, right $1 \%$ ). Averages were computed relative to a $200 \mathrm{~ms}$ pre-cue baseline for cue-related ERPs and relative to a 200 ms pre-stimulus baseline for ERPs elicited after onset of the peripheral stimulus.

\section{Behavioral analysis}

Reaction time validity effects were analyzed separately for arrow and line tasks using a mixed design analysis of variance (ANOVA). There were three experimental factors manipulated within subjects: validity (3: valid, neutral, invalid), stimulus position (2: left, right), and SOA (2: 617, 917). Instructed mapping (2: arrow: congruent or incongruent; line: short-left or short-right) was the only between factor.

\section{ERP analysis}

ERPs elicited by peripheral stimuli. Modulation of the P1 (160-210 ms) and N1 (210-250 ms) by attended versus unattended peripheral stimuli were analyzed. Mean voltages within these windows at electrodes P7 and P8 were used as dependent

1 Channels FP1/FP2 and T7/T8 were excluded from the analysis because of frequent blink-related saturation of the amplifier, and bad electrode-head contact, respectively.). 
variables. In the analysis, instructed mapping (2: arrow: congruent or incongruent; line: short-left or short-right) was included as between factor and within factors were validity (2: valid, invalid), SOA (2: 617, 917), stimulus position (2: left, right), and hemisphere (2: left, right). ${ }^{2}$

Cue ERPs. The latency window between 0 and $617 \mathrm{~ms}$ relative to cue onset was analyzed. For the long SOA trials, it could have been extended until $917 \mathrm{~ms}$, but the use of a short window enabled pooling the short and long SOA trials. To decrease the number of tests, the window was divided into three successive latency windows (150-250 ms; 300-500 ms; 500-600 ms) based on previous findings (Harter et al., 1989; Jongen, Smulders, \& van Breukelen, 2006; Mangun, 1994; Van Velzen \& Eimer, 2003). Mean voltages within these windows were used as dependent variables in three separate regional analyses. The three regions consisted of anterior (F7/8, F3/4), central (FC5/6, $\mathrm{C} 3 / 4, \mathrm{CP} 5 / 6$ ) and posterior (P3/4, P7/8, P9/10, PO7/8, O1/2) sites. Between 300 and 500 $\mathrm{ms}$, a negativity was expected at frontal and central sites (ADAN) and at posterior sites (EDAN). Between 150 and $250 \mathrm{~ms}$, a positivity was expected at posterior sites because it was found in a comparable experiment with arrow cues conducted in our lab (Jongen, Smulders, \& van Breukelen, 2006). Finally, at posterior sites a late positivity (500-600 ms; LDAP) was expected. ERP mean amplitudes were analyzed in a mixed design ANOVA. There were three within factors: cued direction of attention (2: left, right), hemisphere (2: left, right), and electrode (2, 3 or 5 , depending on the region). Instructed mapping (2: arrow: congruent or incongruent; line: short left or short right) was the only between factor. In these analyses, the presence of a lateralized effect sensitive to the direction of an attentional shift would be reflected by the interaction between the cued direction of attention and hemisphere. Interactions between any other factor and cue and hemisphere will be assessed separately for every level of this other factor. For each homologous electrode pair, ERP lateralizations sensitive to the direction of attention were calculated by subtracting, for each cue direction, ERPs at the ipsilateral hemisphere from ERPs at the contralateral hemisphere, and then summing these difference waves of both cue directions. In addition to the time series at different electrode positions, the distribution of the ERP lateralizations across the scalp was studied using

2 The effect of validity (Valid minus invalid) is often examined separately for the hemispheres that are directly and indirectly stimulated by the peripheral target. In that case, however, validity effects on target processing are confounded with cue-related lateralizations (e.g. LDAP): First, for the directly stimulated hemisphere, the difference between validly and invalidly cued targets amounts to the difference between trials with a cue pointing toward its receptive hemifield and trials with a cue pointing away from its receptive hemifield. It is possible that some cue-related lateralization still remains during the baseline of the target or even after the target. This lateralization then confounds validity effects on target processing. Second, for the indirectly stimulated hemisphere, receptive hemifields are reversed. In this case, the confound by the cue-related lateralization has the same magnitude, but the opposite sign. Therefore, pooling across hemispheres that are directly and indirectly stimulated by the target will average out any lateralized cueing effect in the cue-target interval (such as the LDAP) that confound the pre-target baseline and whose resolution ('going back' to baseline) also confounds post-target ERPs even if their baseline is taken just prior to target onset. With our factorial design, we therefore refrain from interpreting any interaction between the validity effect and target-stimulated hemisphere (direct, indirect). 
the current source density (CSD; BESA). For this, lateralized potentials were projected (arbitrarily) on the right side of the head and polarity-inverted waveforms were copied onto the homologous electrode sites on the left side (e.g. Praamstra, Cools, Horstink, \& Stegeman, 1996; Van der Lubbe, Neggers, Verleger, \& Kenemans, 2006).

In all analyses, the Greenhouse-Geisser epsilon correction factor was applied to compensate for possible effects of non-sphericity. The corrected F- and probability values and the uncorrected degrees of freedom are reported.

\section{Results}

An alpha level of .05 was maintained for all statistical tests. Degrees of freedom were $(1,22)$ unless stated otherwise.

\section{Behavioral performance}

A comparison of the percentage of errors in the line and arrow tasks, showed no differences in the percentage of misses, false alarms, responses on the cue, or outliers (all $t$ (23)s $<1.3$, all $p s>.20$ ). A mutual comparison of the two arrow and the two line mappings also showed no differences between the arrow mappings (all $F \mathrm{~s}<2.3$, all $p \mathrm{~s}>$ .14 ), or the line mappings (all $F \mathrm{~s}<1.4$, all $p s>.24$ ). On average the percentage of false alarms ranged from 0 to $16.7 \%$ (average $3.3 \%$ ), of responses on the cue from 0 to $5.4 \%$ (average 1.6\%), of misses from 0 to $4.2 \%$ (average $<1 \%$ ), and of outliers ranged from 0 to 3.8 (average $0.82 \%$ ). After exclusion of trials that contained horizontal eye movements, EEG-artefacts, errors, or outliers, $94.4 \%, 91.1 \%, 92.8 \%$ and $93.7 \%$ of all trials remained for analyses of the congruent arrow, incongruent arrow, short-right line, and short-left line mapping, respectively.

Table 3.1 displays average response times as a function of task and instructed mapping. Data of the line task are collapsed over the two line mappings, because the distinction between them was not of prime interest, and they did not exert an effect on the behavioral or ERP results. In the arrow task, responses were faster for stimuli to the right (305 ms) than for stimuli to the left (315 ms), $F=14.0, p=.001$. This might be related to the fact that all responses were right-handed. Responses were faster after the long (307 ms) than after the short (314 ms) SOA, $F=22.6, p<.0005$. In addition, there was a main effect of validity, $F(2,21)=32.6, p<.0005$. Responses to validly cued stimuli were faster ( $286 \mathrm{~ms})$ than to neutrally (308 ms) or invalidly cued (336 ms) stimuli. There were significant costs (invalid versus neutral), $F=28.8, p<.0005$, and benefits (valid versus neutral) of attention, $F=55.0, p<.0005$. The main effect of validity was modified by interactions between validity and stimulus position, $F(2,21)=$ 4.6, $p=.02$, and between validity and SOA, $F(2,21)=4.3, p=.03$. Separate analyses showed that there were validity effects for stimulus left $(F(2,21)=26.7, p<.0005)$ and stimulus right $(F(2,21)=34.5, p<.0005)$ trials, but costs were larger for stimulus left, $F=8.6, p=.01$. Concerning the interaction with SOA, separate analyses showed a validity effect for the short $\operatorname{SOA}(F(2,21)=28.9, p<.0005)$ and the long $\operatorname{SOA}(F(2$, $21)=26.8, p<.0005)$, but costs were smaller for the long SOA, $F=6.2, p=.02$.

Similar to the results from the arrow task, responses in the line task were faster for stimuli to the right $(301 \mathrm{~ms})$ than for stimuli to the left $(307 \mathrm{~ms}), F=9.4, p=.006$. More important, a main effect of validity, $F(2,21)=20.6, p<.0005$ showed that participants effectively used the line cues despite the fact that these had no intrinsic 
directional properties. Responses to validly cued stimuli were significantly faster (289 $\mathrm{ms})$ than to neutrally $(301 \mathrm{~ms})$ or invalidly $(322 \mathrm{~ms})$ cued stimuli. There were significant costs, $F=23.6, p<.0005$, and benefits, $F=21.0, p<.0005$. There was an interaction between validity and SOA, $F(2,21)=4.8, p=.02$. Separate analyses showed a significant validity effect for the short $\operatorname{SOA}(F(2,21)=24.6, p<.0005)$ and the long SOA $(F(2,21)=12.5, p<.0005)$, but benefits were smaller for the longer SOA $(F=$ $6.0, p=.02)$.

Table 3.1 Mean RT (ms) and standard error (in parentheses) for the different task conditions in experiment 1 in short and long SOA trials for stimulus left and stimulus right as a function of the cued direction of attention.

$$
\mathrm{SOA}=617 \mathrm{~ms} \quad \mathrm{SOA}=917 \mathrm{~ms}
$$

\begin{tabular}{|c|c|c|c|c|c|}
\hline \multirow[t]{2}{*}{ Task condition } & \multirow[t]{2}{*}{ Cued direction } & \multicolumn{2}{|c|}{ Stimulus position } & \multicolumn{2}{|c|}{ Stimulus position } \\
\hline & & Left & Right & Left & Right \\
\hline \multicolumn{6}{|l|}{$\begin{array}{r}\text { Congruent } \\
\text { Arrow }\end{array}$} \\
\hline & Left & $284(10)$ & 344 (15) & $290(11)$ & 333 (14) \\
\hline & Neutral & 317 (13) & 302 (12) & $310(14)$ & $298(11)$ \\
\hline & Right & $368(22)$ & $283(10)$ & 343 (19) & $276(8)$ \\
\hline & Benefits & $33(7)$ & $19(6)$ & $20(4)$ & $22(4)$ \\
\hline & Costs & $51(11)$ & $42(9)$ & 33 (14) & $35(9)$ \\
\hline \multicolumn{6}{|l|}{$\begin{array}{r}\text { Incongruent } \\
\text { Arrow }\end{array}$} \\
\hline & Left & $293(14)$ & $326(15)$ & 289 (12) & 304 (14) \\
\hline & Neutral & $313(17)$ & $313(17)$ & 305 (17) & 309 (17) \\
\hline & Right & $340(16)$ & $286(15)$ & 333 (19) & $290(15)$ \\
\hline & Benefits & $20(7)$ & $27(4)$ & $16(7)$ & $19(5)$ \\
\hline & Costs & $27(4)$ & $13(10)$ & $28(7)$ & $-4.6(7)$ \\
\hline \multicolumn{6}{|l|}{ Line } \\
\hline & Left & $293(10)$ & $326(12)$ & 293 (9) & $310(10)$ \\
\hline & Neutral & 305 (1 1) & 303 (10) & $301(10)$ & $296(10)$ \\
\hline & Right & 330 (11) & $286(9)$ & $322(10)$ & $285(8)$ \\
\hline & Benefits & $12(4)$ & 17 (3) & $8(3)$ & $11(3)$ \\
\hline & Costs & $25(5)$ & $22(5)$ & $21(7)$ & $14(5)$ \\
\hline
\end{tabular}

Finally, validity effects of line and arrow tasks were compared in a repeated measures ANOVA with task (2: arrow, line), validity (3: valid, neutral, invalid), stimulus position (2: left, right), and SOA (2: 617, $917 \mathrm{~ms})$ as within factors. There was an interaction between validity and task, $F(2,22)=5.8, p=.009$. Whereas costs were similar, $F(1,23)$ 
$=1.5, p=.23$, benefits were smaller for the line than for the arrow tasks, $F(1,23)=$ $11.6, p=.002$.

In sum, RT results confirmed that attention was oriented in response to all cues. Although there was no difference between the two arrow-mappings or between the two line-mappings, benefits but not costs were smaller in the line task than in the arrow task.

\section{ERP data}

ERPs elicited by peripheral stimuli. Figure 3.2a shows the effect of validity on the P1 and N1 response to peripheral stimuli as a function of task and instructed mapping. Data of the line task are collapsed again over the two mappings. Both components were small in all task mappings for both validly and invalidly cued stimuli. In the arrow task, $\mathrm{P} 1$ but not $\mathrm{N} 1$ was larger on valid than invalid trials $(F=6.3, p=.02$; $F<1, p=.82$, respectively). In the line task, neither the $\mathrm{P} 1(F<1, p=.79)$ nor the N1 $(F<1, p=.34)$ showed a validity effect.

Cue ERPs. The main aim of the present study was to find out if there were lateralized ERP components in the congruent arrow mapping and whether these would extend to the incongruent mapping and the line task. Results are reported separately for arrow and line task data, and for various scalp regions.

Figure 3.3a and 3.3b show ERPs in response to attend-left and attend-right cues for the congruent and incongruent mapping. Figure 3.4 shows difference waves for the congruent and incongruent mapping of the arrow task. Orienting-related contralateral negativity (positivity) is reflected in these waves by a negative (positive) amplitude. In addition, Figure 3.8 shows CSD maps for the difference waves at the moment when components peak or in intervals where they were expected. At posterior sites, between 150 and $250 \mathrm{~ms}$, there was a lateralized early positive component in the congruent mapping (peak latency 206-226 ms, dependent on electrode position) and a negative component in the incongruent mapping (peak latency 190-198 ms), as indicated by an interaction between cue, hemisphere and mapping, $F=39.2, p<.0005$. Further analyses showed a significant interaction between cue and hemisphere for both mappings (congruent: $F(1,11)=16.5, p=.002$; incongruent: $F(1,11)=26.4, p<.0005$ ). The reversal of this component in the incongruent mapping implies that it is sensitive to the physical direction of the arrow rather than the direction of attention. This is in accordance with the idea that it reflects sensory processing of the slightly asymmetric cues. This early component was followed by a lateralized negative component between 300 and $500 \mathrm{~ms}$ in both mappings (peak latency: congruent 316-364 ms; incongruent 376-384 ms), reflecting an EDAN. This was confirmed by a significant interaction between cue and hemisphere, $F=25.6, p<.0005$. In the same interval, there was an interaction between cue, hemisphere and electrode $F(4,88)=6.3, p=.001$, reflecting that this effect was strongest at PO7/8, P9/10 and P7/8. The EDAN was followed by a lateralized positive component, reflecting a LDAP between 500 and $600 \mathrm{~ms}, F=4.6$, $p=.04$. However, there was a difference between the two mappings as shown by an interaction between cue, hemisphere and mapping, $F=8.2, p=.009$. Separate analyses for every mapping showed there only was a significant LDAP in the congruent mapping (congruent: $F(1,11)=9.5, p=.001$, peak latency $595 \mathrm{~ms}$; incongruent: $F(1,11)<1$, $p=.54)$. Finally, in the same interval, there was an interaction between cue, hemi- 


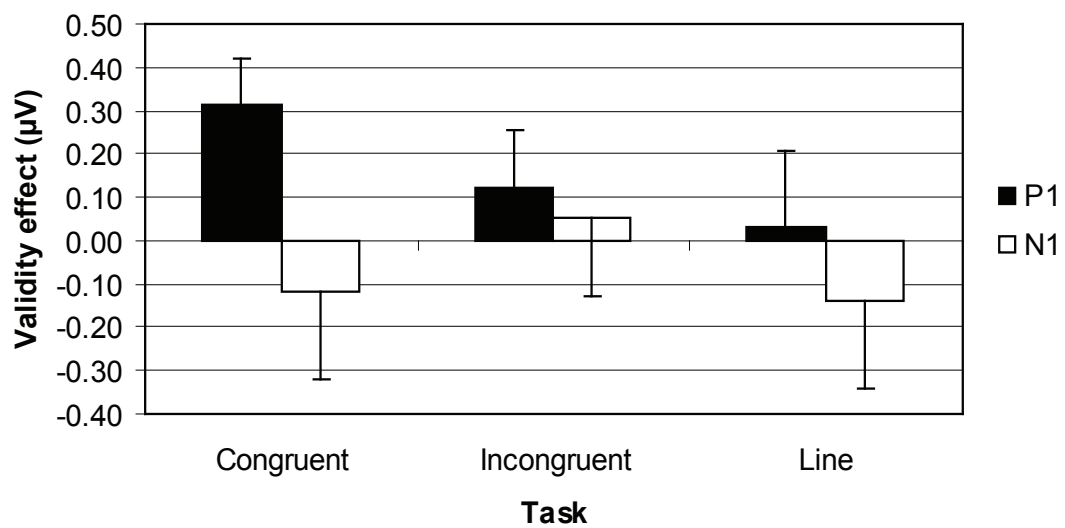

b

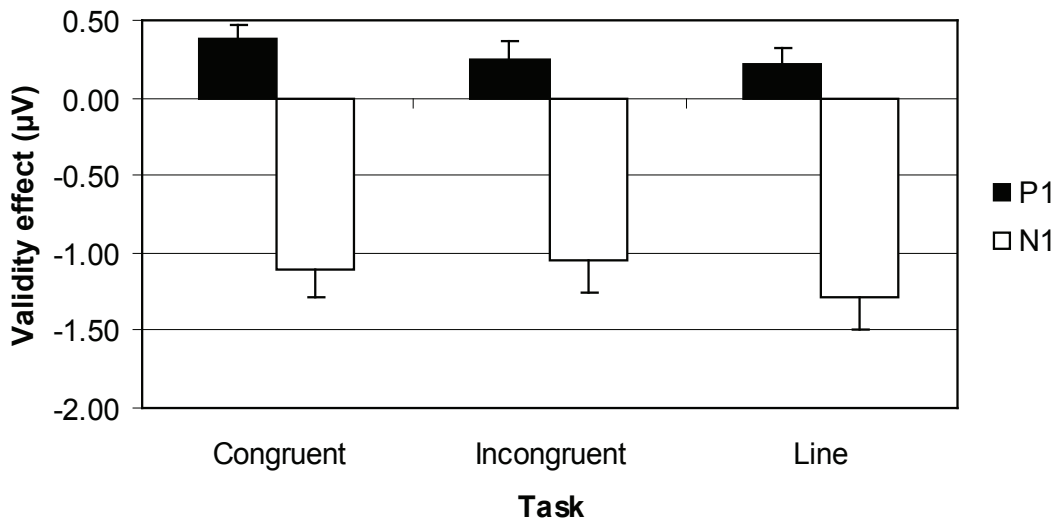

Figure 3.2 Bar graphs showing the validity effect on the amplitudes of the P1 and N1 components to the peripheral stimuli in the different tasks for (a) Experiment 1 and (b) Experiment 2. Area measures of the difference between ERPs to valid and invalid trials (valid minus invalid) were taken for the $\mathrm{P} 1$ and $\mathrm{N} 1$ component at P7/P8. Data are pooled for SOA, stimulus position, and hemisphere. Validity effects were computed as 'valid'-'invalid', so attention-related enhancement of $\mathrm{Pl}$ and $\mathrm{Nl}$ amounts to a positive and negative validity effect, respectively.

sphere and electrode $F(4,88)=3.1, p=.04$, reflecting that the LDAP was strongest at P3/4 and 01/2. Note that these are the same electrodes where the EDAN was weaker which suggest that there is an inverse relationship between these components probably due to their opposite polarity and spatial and temporal overlap. 
a

Experiment 1, congruent arrow mapping
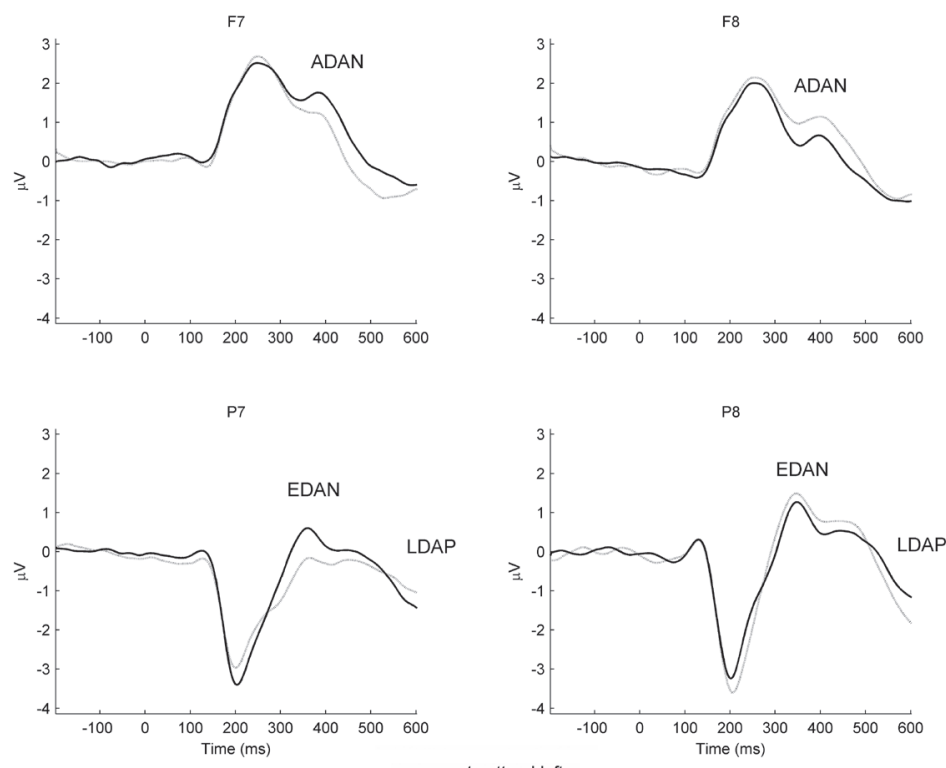

- cue to attend left
cue to attend right

b

Experiment 1, incongruent arrow mapping

F7

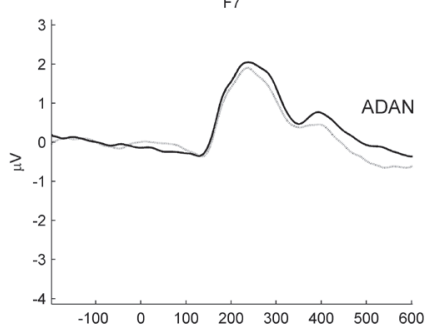

P7

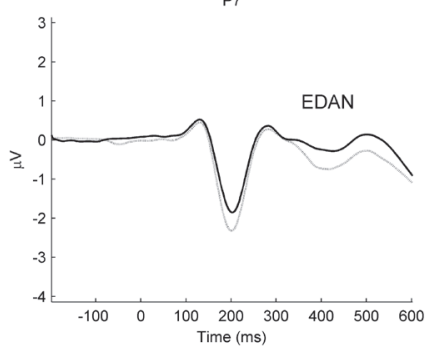

F8

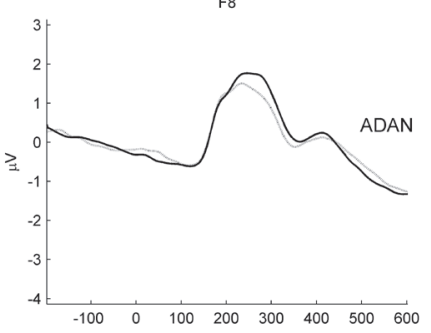

P8

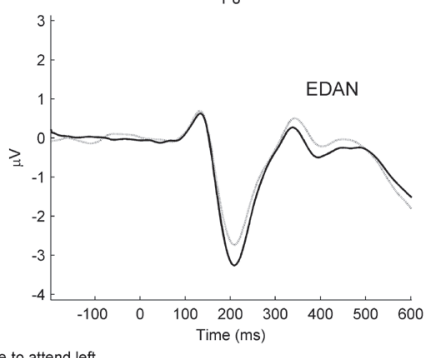

- cue to attend left
cue to attend right 

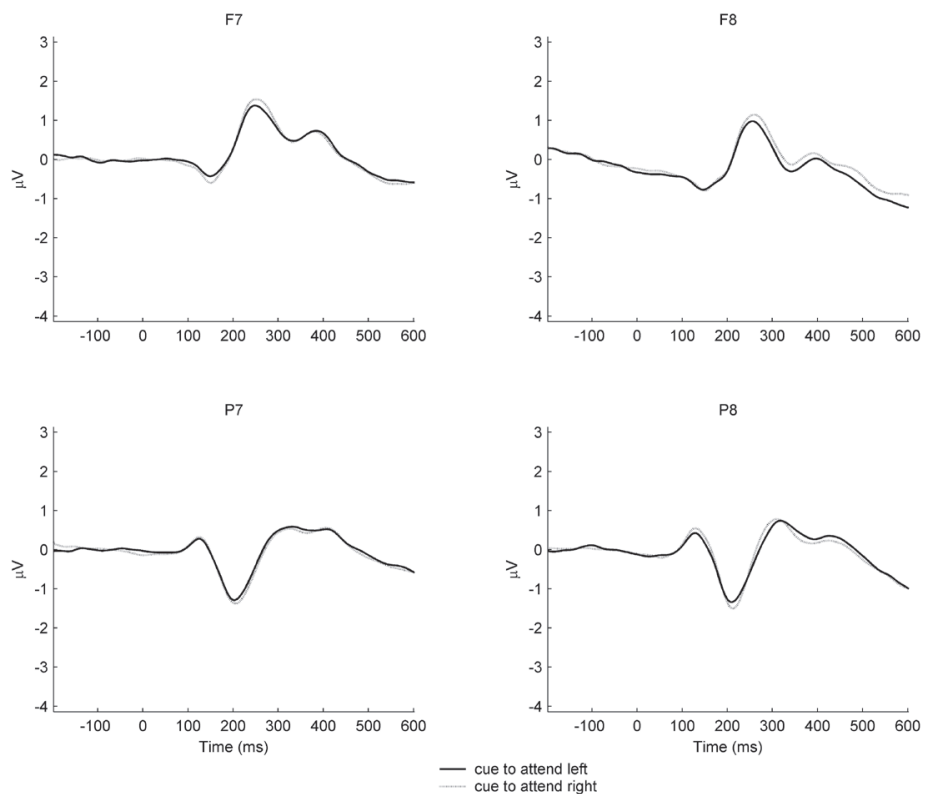

Figure 3.3 Grand-averaged ERPs at two selected electrode pairs, in the interval between cue onset and onset of the peripheral stimulus in the (a) congruent arrow mapping, (b) incongruent arrow mapping, and (c) line task of Experiment 1. Three components contralateral to the cued direction of attention are indicated: an anterior negativity (ADAN), a posterior negativity (EDAN) and a posterior positivity (LDAP). At these electrode pairs, there were no lateralized components in the line task. 


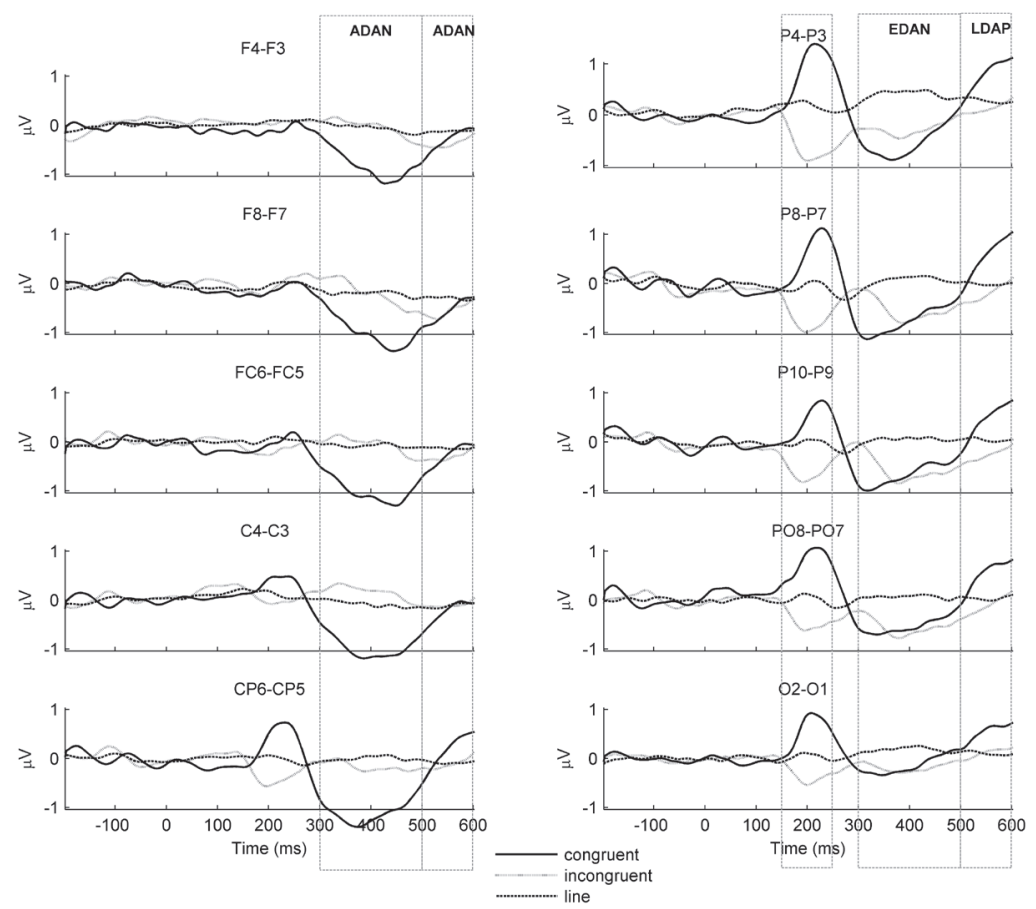

Figure 3.4 Grand-averaged ERP difference waves for all homologue electrode pairs in the cuetarget interval (for short SOA trials the peripheral stimulus was presented after $617 \mathrm{~ms}$ ) in the congruent and incongruent arrow mapping, and the line task of Experiment 1. Data are pooled for SOA. Negative components contralateral to the cued direction of attention are reflected by negative values, positive components are reflected by positive values. The time window in which the amplitude of the lateralized difference was statistically tested is shown by dashed boxes and expected components are indicated. An additional time window was tested for the anterior negativity in the arrow task. F-values and significance are described in the text.

At central and frontal sites, between 300 and $500 \mathrm{~ms}$, there was a lateralized negativity, reflecting an ADAN, as indicated by an interaction between cue and hemisphere at frontal, $F=17.2, p<.0005$, and central sites, $F=18.3, p<.0005$. However, as shown in Figure 3.4, in the incongruent mapping, this component seems smaller and shifted in latency at frontal sites while absent at central sites. This was indicated by a significant interaction between cue, hemisphere and mapping at frontal, $F=9.8, p=$ .005 , and central sites, $F=16.7, p<.0005$. Further analyses showed an ADAN between 300 and $500 \mathrm{~ms}$ only in the congruent mapping (frontal: $F(1,11)=19.4, p=.001$, peak latency 426-442 ms; central: $F(1,11)=23.7, p<.0005$, peak latency 372-446 ms), and not in the incongruent mapping (frontal: $F(1,11)<1, p=.40$; central: $F(1,11)<1$, $p=.90)$. To measure the latency shift in the incongruent mapping, an additional analysis with the same factors was done between 500 and $600 \mathrm{~ms}$. This revealed a significant interaction between cue and hemisphere, $F(1,22)=14.5, p=.001$, indicating that an ADAN was also elicited in the incongruent mapping, but somewhat later than in the congruent mapping (peak latency 520-540 ms). If the LDAP also was elicited with a delay in the incongruent mapping, the interval used for analysis (500-600 ms) would be 
too short to examine it. In an extra analysis long SOA trials were therefore examined to exclude the possibility of a latency shift. Difference waves are presented in Figure 3.5 and show that similar to the pooled average of short and long SOA trials there was an LDAP only in the congruent mapping. This was confirmed by statistics between 500 and $700 \mathrm{~ms}$ that showed an interaction between cue and hemisphere, $F=14.9, p=.001$, and between cue, hemisphere and mapping, $F=14.0, p=.001$. Separate analyses showed an LDAP only in the congruent mapping (congruent: $F(1,11)=19.5, p=.001$; incongruent: $F(1,11)<1, p=.91)$.

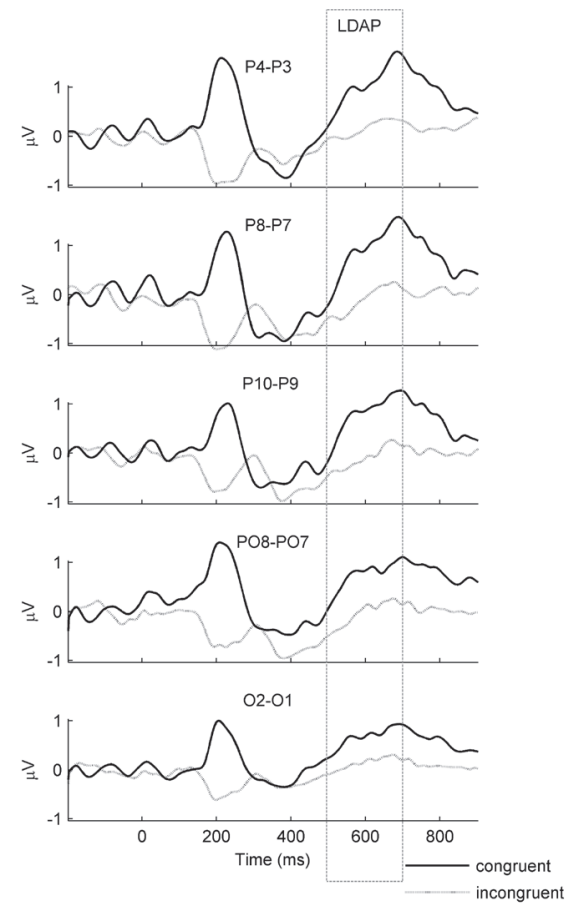

Figure 3.5 Grand-averaged ERP difference waves for long SOA trials for all homologue electrode pairs in the cue-target interval in the congruent and incongruent arrow mapping of Experiment 1. Negative components contralateral to the cued direction of attention are reflected by negative values, positive components are reflected by positive values. A posterior positivity (LDAP) was expected and statistically tested in the interval that is indicated by the dashed box.

Figure 3.3c shows ERPs in response to attention-left and attention-right line cues and Figure 3.4 shows difference waves for the line task. In addition, CSD maps for the difference waves are given in Figure 3.8. Data are collapsed over the short-left and short-right mapping, because the distinction between them was not of prime interest, and mapping did not exert an effect on the behavioral or ERP results. A shown by the difference waves in Fig.4, there was almost no lateralized activity elicited. Statistics showed, there only was an interaction between cue, hemisphere and electrode, at posterior sites, between 300 and $500 \mathrm{~ms}, F(4,88)=3.0, p=.04$. Further analyses showed a positive component at $01 / 2(F(1,22)=9.0, p=.01)$ and $\mathrm{P} 3 / 4(F(1,22)=6.4, p=.04)$ (peak latency $416-430 \mathrm{~ms}$ ). Note that the LDAP in the congruent mapping was strongest at these posterior positions, which suggests that this might be an early LDAP.

In sum, for the arrow data the interaction between cue and hemisphere reflected a number of lateralized components. First of all, at posterior sites between 150 and $250 \mathrm{~ms}$ a positive component in the congruent mapping was mirrored by a negative component in the incongruent mapping. Second, at posterior sites in the congruent 
and the incongruent mapping there was a negative component between 300 and 500 ms reflecting an EDAN. Third, at frontal and central sites there was an ADAN between 300 and $500 \mathrm{~ms}$, and at posterior sites there was a LDAP between 500 and $600 \mathrm{~ms}$, and these were elicited only in the congruent mapping. In the incongruent mapping, there was a latency shift for the ADAN at frontal sites where it was elicited between 500 and $600 \mathrm{~ms}$. In the line task there only was a positivity at some of the posterior electrodes between 300 and $500 \mathrm{~ms}$, possibly reflecting an early LDAP.

\section{Discussion}

A number of lateralized components were elicited after cue-onset in the congruent and incongruent arrow mapping. A comparison of these two mappings was made to draw conclusions on the functional significance of these components. The results showed an early positive component between 150 and $250 \mathrm{~ms}$ at posterior sites in the congruent mapping that reversed in polarity when the direction of the arrow, but not the direction of attention, changed. This reversal and the early latency suggest that it is a reflection of sensory processing of the cue. Following this early component, at posterior sites between 300 and 500 ms there was an EDAN that did not reverse in amplitude between the two mappings. This suggests that it reflects processes of orienting. At frontal sites there was an ADAN between 300 and $600 \mathrm{~ms}$ in the congruent mapping. Although there was no reversal of amplitude in the incongruent mapping, there was a latency shift and the component was elicited between 500 and $600 \mathrm{~ms}$. This might be due to the unnatural attentional response -against the direction of the arrow- that is requested in the incongruent mapping. At central sites, there was an anterior negativity only in the congruent mapping. Similarly, there was an LDAP at posterior sites only in the congruent mapping. The possibility that this was due to a latency shift in the incongruent mapping was excluded by analyzing long SOA trials where it was shown to be also absent. Taken together, the results of the arrow task suggest that only the ADAN and the EDAN are consistently related to processes of attentional orienting. In a second task, the physical shape of the cues was controlled by presenting nonlateralized line cues. A positivity was elicited at a number of posterior electrodes between 300 and $500 \mathrm{~ms}$, possibly reflecting an early LDAP.

Reaction time results showed costs and benefits of attention in both the arrow and the line tasks. However, modulation of early sensory effects elicited after the peripheral stimulus was only shown in the arrow tasks, and only the P1 component was modulated. A similar pattern of results has been reported in other studies (e.g Eimer, 1993; Eimer, 1994; Mangun \& Hillyard, 1991). Similar to the results in the arrow task presented here, Mangun and Hillyard (1991) reported RT validity effects and modulation of the P1 but not the N1 when a simple detection task was used. When the task was changed and participants were asked to discriminate the peripheral stimulus, both components were modulated by attention. Therefore, it was concluded that discrimination might have required further engagement of attention to the stimulus. Modulation in a discrimination paradigm was shown to depend on the level of perceptual load (Eimer, 1993; Handy \& Mangun, 2000). That is, despite RT validity effects, there was no modulation in a discrimination task (Eimer, 1993), whereas both components were modulated when the level of difficulty was increased. The level of modulation also has 
been shown to depend on the relevancy of the uncued position (Eimer, 1994; Mangun \& Hillyard, 1990). Systematic manipulation of the relative allocation of attention to the left and right position was reflected in differential modulation of the P1 and N1 amplitude (Mangun \& Hillyard, 1990). Eimer (1994) directly compared a simple detection task with a task in which participants were required to respond only to validly cued stimuli. Whereas both components were modulated in the latter, only the N1 was modulated in the simple detection task. It was suggested that asking participants to respond only to validly cued stimuli might lead them to ignore the uncued side. Attention might therefore be more focused at the cued side than in a simple detection task where it may alternatively be more divided between possible stimulus locations.

Taken together, these studies have shown that attentional modulation of early sensory components elicited after onset of peripheral stimuli depends on task demands and on the relevancy of the uncued position. In the present study there were RT validity effects in both arrow mappings and the line task but modulation of early components after onset of the peripheral stimulus was weak in the arrow mappings and absent in the line task. In addition there was almost no lateralized activity after cue onset in the line tasks and only weak activity in the incongruent as compared to the congruent arrow mapping. Although this shows a discrepancy between RT validity effects and ERP results after cue and stimulus onset, it suggests a relation between modulation of early components after onset of the peripheral stimulus and lateralized cue ERPs. In the second study, it was attempted to augment lateralized cue ERPs by employing a go-nogo attention task, known to demonstrate stronger attention effects on ERPs after stimulus onset than the all-go variant of experiment 1.

\section{EXPERIMENT 2}

In comparison with the all-go detection task that was used in the first experiment, attentional modulation may be enlarged in a discrimination task or in a task that would have required responses only to validly cued, infrequent target stimuli. A combination of both manipulations would increase attentional demands and attentional focus and was applied in the second experiment.

\section{Method}

\section{Participants}

Twenty-seven volunteers participated in the study. Three were discarded from the analyses because of poor eye fixation in the cue-target interval. Thus, 24 participants (age 18-42, mean age 24, 15 female) remained. Twenty participants were right-handed, and all had (corrected to) normal vision. All participants gave informed consent and received course credits or were paid for their participation.

\section{Behavioral tasks and procedure}

The same room and settings were used, and the procedure was also roughly similar to experiment 1 . Only differences will be described. First, the task was changed into a discrimination task. The peripheral stimulus was either a vertically or a horizontally oriented black-and-white grating $\left(2.2^{\circ}, 7.3\right.$ cycles/degree $)$ and participants were instructed 
to only respond to horizontally oriented gratings by pressing a response button with their right index finger. Discrimination of the peripheral stimulus should increase attentional demands. Second, participants were asked only to respond to validly cued target stimuli because this was suggested to increase attentional focus to the cued side. For the same purpose lines similar to the fixation line were located at the center of both stimulus positions and served as landmarks. Third, the design was changed into a full within-subject design by having every subject participate in all of the instructed mappings. The order of tasks and instructed mappings was balanced across participants and mappings from the arrow and line task always alternated (arrow-line-arrow-line; line-arrow-line-arrow). This was done to minimize the interference from switching the instructed mapping. The peripheral stimulus was presented for $150 \mathrm{~ms}$ which is slightly longer than in experiment 1 because of the discriminative response. The blank interval after cue presentation always was $617 \mathrm{~ms}$. Neutral cues would have been meaningless and were omitted. Cues indicating the left or right stimulus position always had a predictive validity of $50 \%$. Target stimuli had a horizontal orientation and were presented in $20 \%$ of all trials. Therefore, in $10 \%$ of all trials targets were validly cued and required a response.

Every experimental block consisted of 4 "warm-up" trials and 400 trials to be analyzed and was preceded by a 60-trial practice block. Participants received feedback about their reaction time after every 50 trials and could proceed with the task when they felt ready. After participants had participated in two mappings, they were given a 10-minute break.

\section{Data recording}

Amplifier band pass was set to $0.05-30 \mathrm{~Hz}$, and digitization rate was $250 \mathrm{~Hz}$. Only HEOG data were re-filtered offline, with the same settings as in experiment 1 . Epochs were made starting $200 \mathrm{~ms}$ before cue onset and ending $400 \mathrm{~ms}$ after onset of the peripheral stimulus. Trial removal criteria were similar to experiment 1 . This led to the exclusion of three participants, and the residual average percentage of HEOG activity in all participants included in the analyses was $2.2 \%$ for the arrow task (left $2 \%$, right $2.4 \%$ ) and $1.5 \%$ for the line task (left $1.3 \%$, right $1.6 \%$ ).

\section{ERP analysis}

ERPs elicited by peripheral stimuli. Modulation of the P1 (125-175 ms) and N1 (200-250 ms) by attended versus unattended non-target stimuli were investigated. Mean voltages within these windows at electrodes P7 and P8 were used as dependent variables. Amplitude values were calculated with reference to a $200 \mathrm{~ms}$ pre-stimulus baseline. Order of the two mappings of every task was included as between-factor. Within-factors were instructed mapping (2: congruent and incongruent; short-left and short-right), validity (2: valid, invalid), stimulus position (2: left, right), and hemisphere (2: left, right).

Cue ERPs. The three successive latency windows were similar to experiment 1 , only the window for the LDAP was broader (500-700 ms). Averages were computed relative to a $200 \mathrm{~ms}$ pre-cue baseline. Order of the two mappings of a task was included as between-factor. Within-factors were instructed mapping (2: congruent and incongruent; short-left and short-right), cue (2: left, right), hemisphere (2: left, right) and electrode $(2,3$, or 5 , depending on the region). 


\section{Results}

An alpha level of .05 was maintained for all statistical tests. Degrees of freedom were (1, 22) unless stated otherwise. Effects of the factor order are not described because the factor had no important effects and none of the critical effects depended on it.

Behavioral performance

There was no difference between the four conditions in the percentage of RT-outliers (average 1.59\%), $F(3,21)<1$, misses (average 5.6\%), $F(3,21)=1.4, p=.27$, false alarms (average $.56 \%), F(3,21)=2.8, p=.07$ or responses on the cue (average $<1 \%$ ), $F(3,21)=2.3, p=.10$. A comparison of line tasks and arrow tasks showed that there were slightly more false alarms in the line tasks than in the arrow tasks, $F(1,23)=4.2$, $p=.05$. However, in all tasks the percentage of false alarms was $<1 \%$. After exclusion of trials with eye movements, EEG artefacts, errors or outliers, $91.9 \%, 91.6 \%, 91.9 \%$ and $91.8 \%$ of all trials remained for analyses of the congruent arrow mapping, incongruent arrow mapping, short-right line mapping, and short-left line mapping, respectively. There was no difference in reaction times between the congruent $(510 \mathrm{~ms})$ and incongruent (509 ms) arrow mapping, or between the short-left (513 ms) and short-right (510 $\mathrm{ms}$ ) line mapping, but similar to experiment 1 responses were faster for stimuli to the right in the arrow (right: $503 \mathrm{~ms}$, left: $516 \mathrm{~ms}$ ) and line (right: $504 \mathrm{~ms}$, left: $519 \mathrm{~ms}$ ) task (arrow: $F=7.8, p=.01$; line: $F=8.5, p=.01$ ).

\section{ERP data}

ERPs elicited by peripheral stimuli ERPs. Figure $3.2 \mathrm{~b}$ shows stimulus related effects for the arrow mappings and the line task. Main effects of validity showed that $\mathrm{P} 1$ and $\mathrm{N} 1$ were modulated by attention in the expected direction in the arrow (P1: $F=12.2, p=.002, \mathrm{~N} 1: F=45.1, p<.0005)$ and the line task $(\mathrm{P} 1: F=5.0, p=.04$, and N1: $F=44.9, p<.0005)$. The size of the N1 validity effect in the two line mappings for stimulus left and stimulus right was different, as indicated by an interaction between instructed mapping, validity, and stimulus position, $F=7.4, p=.01$. However, separate tests for every mapping showed a significant $\mathrm{N} 1$ validity effect in both mappings (short-left: $F=42.8, p<.0005$; short-right: $F=35.9, p<.0005)$. Finally, validity effects on the P1 and N1 component were compared with each other in an ANOVA of task (2: arrow, line), instructed mapping (2: congruent and incongruent, short left and short right), validity (2: valid, invalid), stimulus position (2: left, right) and hemisphere (2: left, right). For both the $\mathrm{P} 1$ and the $\mathrm{N} 1$ effect, there were main effects of validity (P1: $F(1$, $23)=10.7, p=.003 ; \mathrm{N} 1: F(1,23)=52.3, p<.0005)$, but no interaction between task and validity $(\mathrm{P} 1: F(1,23)=1.3, p=.27 ; \mathrm{N} 1: F(1,23)=2.0, p=.17)$.

Cue ERPs. Figure 3.6a and 3.6b show ERPs in response to attend-left and attendright cues for the congruent and incongruent mapping. Difference waves and CSD maps of these are presented in Figure 3.7 and Figure 3.8, respectively. At posterior sites, between 150 and $250 \mathrm{~ms}$, there was an interaction between cue and hemisphere, $F=5.1, p=.03$. However, this interaction was superseded by an interaction between cue, hemisphere and mapping, $F=29.4, p<.0005$, indicating a lateralized early positive component in the congruent mapping (peak latency 196-204 ms) and a negative component in the incongruent mapping (peak latency 188-200 ms). The difference between the congruent and incongruent mapping was larger at P3/4 and PO7/8 than at the 
Experiment 2, congruent arrow mapping
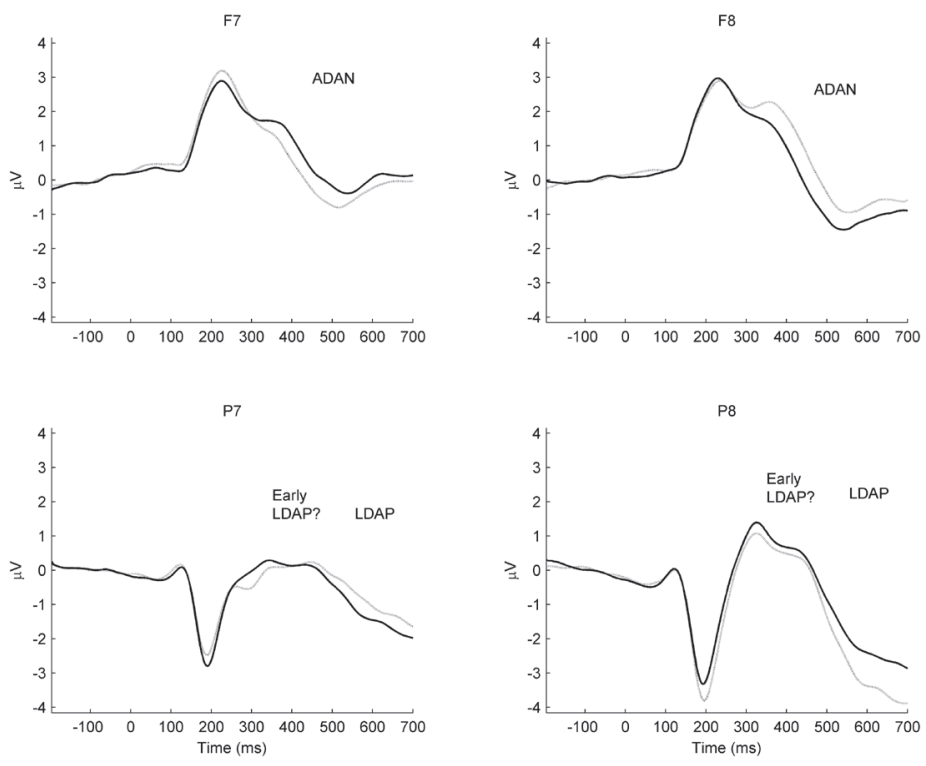

\section{- cue to attend left
cue to attend left}

b

\section{Experiment 2, incongruent arrow mapping}
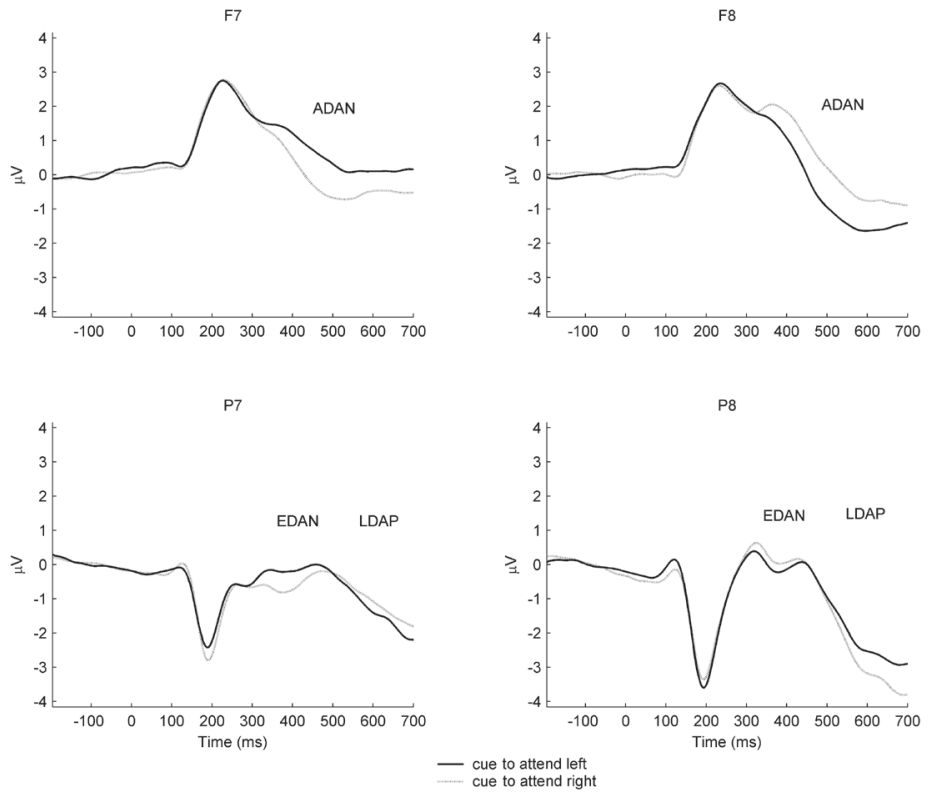
C

Experiment 2, line task
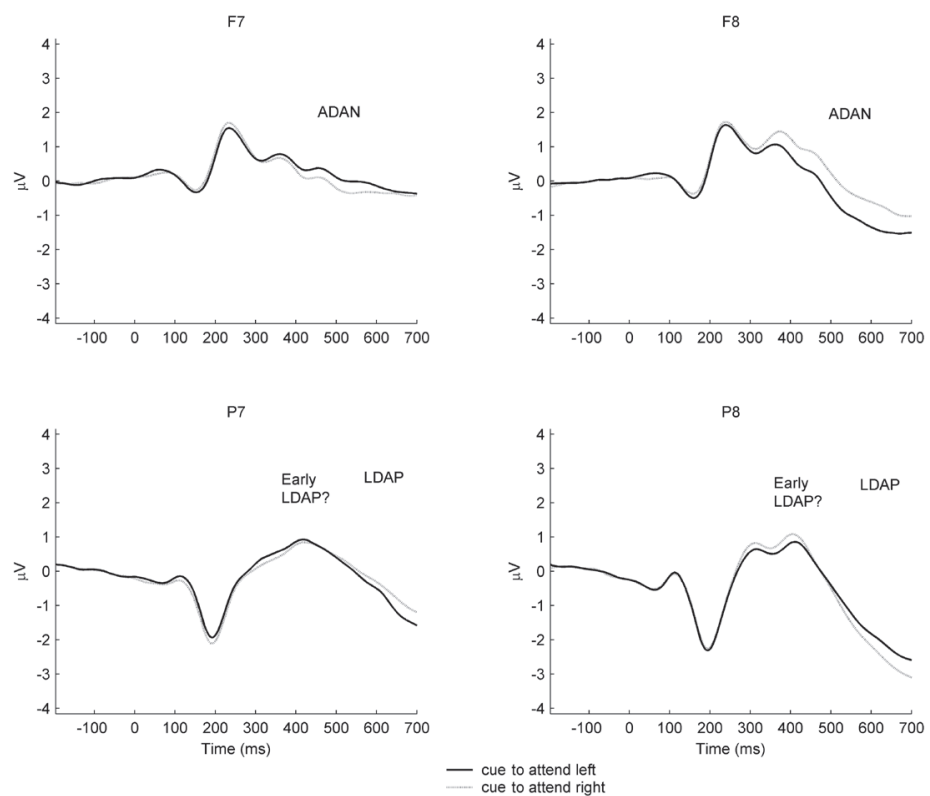

Figure 3.6 Grand-averaged ERPs at two selected electrode pairs, in the cue-target interval in the (a) congruent arrow mapping, (b) incongruent arrow mapping, and (c) line task of Experiment 2. Three components contralateral to the cued direction of attention are indicated: an anterior negativity (ADAN), a posterior negativity (EDAN) and a posterior positivity (LDAP). In the congruent arrow mapping and the line task instead of an EDAN a positive component, perhaps an early LDAP, was found at posterior sites. 


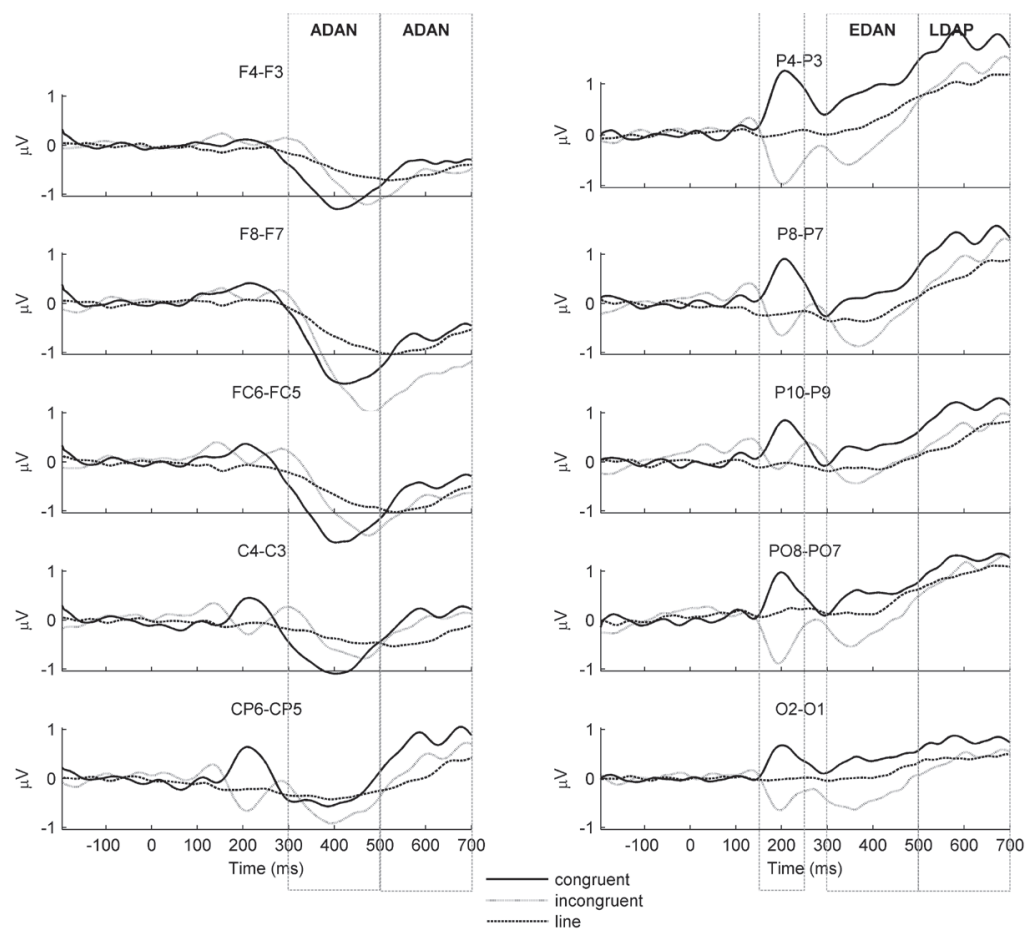

Figure 3.7 Grand-averaged ERP difference waves for all homologue electrode pairs in the cuetarget interval in the congruent and incongruent arrow mapping, and the line task of Experiment 2. Negative components contralateral to the cued direction of attention are reflected by negative values, positive components are reflected by positive values. The time window in which the amplitude of the lateralized difference was statistically tested is shown by dashed boxes and expected components are indicated. An additional time window was tested for the anterior negativity. In the congruent arrow task and the line task instead of an EDAN a positive component, perhaps an early LDAP, was found at posterior sites. F-values and significance are described in the text. In comparison with the congruent mapping, in the incongruent mapping and the line task the ADAN (indicated by the dashed box) is shifted in latency (see text for details).

other electrodes, as shown by an interaction between cue, hemisphere, mapping and electrode, $F(4,88)=9.0, p<.0005$. These early components were followed in time by a lateralized component at posterior sites between 300 and $500 \mathrm{~ms}$, but polarity reversed between the congruent and incongruent mapping, and there was an interaction between cue, hemisphere and mapping, $F(1,22)=15.8, p=.001$. Whereas there was a weak negativity in the incongruent mapping, reflecting an EDAN (peak latency 344$364 \mathrm{~ms}), F=3.4, p=.08$, in the congruent mapping a lateralized positivity occurred, possibly reflecting an early start of the LDAP, $F=11.9, p=.002$. Between 500 and 700 $\mathrm{ms}$, the late positivity was present in both mappings as confirmed by an interaction between cue and hemisphere, $F=22.7, p<.0005$ (peak latency: congruent 576-672 $\mathrm{ms}$; incongruent 680-688 ms). There was an interaction between cue, hemisphere, and mapping $(F=12.0, p=.002)$ and separate tests for every mapping showed that the late positivity was stronger in the congruent $(F(1,11)=43.3, p<.0005)$ than in the incongruent mapping $(F(1,11)=7.1, p=.02)$. 
At central and frontal sites between 300 and $500 \mathrm{~ms}$ an interaction between cue and hemisphere reflected an ADAN (frontal, $F=43.9, p<.0005$; central: $F=15.6$, $p=.001$ ) (peak latency frontal: congruent 404-420 ms, incongruent 468-476 ms; peak latency central: congruent 380-400 ms, incongruent 392-472 ms). As shown in Figure 3.7, at frontal sites these effects were stronger at F7/8 and for the incongruent mapping. This was indicated by an interaction between cue, hemisphere, mapping, and electrode, $F=4.4, p=.048$. At central sites, an interaction between cue, hemisphere, mapping, and electrode $(F(2,44)=11.7, p<.0005)$ reflected that these effects were stronger for the congruent mapping, but only at $\mathrm{C} 3 / 4$ and FC5/6. At frontal sites the ADAN remained significant between 500 and $700 \mathrm{~ms}, F=15.3, p=.001$. There was an interaction between cue, hemisphere and mapping $(F=10.2, p=.004)$ and separate tests showed that the effect was stronger in the incongruent $(F=24.0, p<.0005)$ than in the congruent mapping $(F=5.7, p=.03)$. In addition, there was an interaction between cue, hemisphere, and electrode $(F=13.8, p=.001)$ and separate tests showed that the effect was stronger at F7/8 $(F=17.4, p<.0005)$ than at F3/4 $(F=9.5, p=$ $.005)$. At central sites, there also was an ADAN between 500 and $700 \mathrm{~ms}$, but there was an interaction between cue, hemisphere and electrode $(F(2,44)=34.5, \quad p<.0005)$ and separate tests showed the effect was only significant at the most frontally located electrodes FC5/6 $(F=8.2, p=.01)$.

Figure 3.6c shows ERPs in response to attention-left and attention-right line cues. Difference waves and CSD maps of these are presented in Figure 3.7 and Figure 3.8, respectively. Data are collapsed again for the short-left and short-right mapping, because, as expected, instructed mapping had no effect on ERPs. At posterior sites, there was no early positivity or EDAN, but an LDAP was observed between 500 and $700 \mathrm{~ms}, F=20.2$, $p<.0005$ (peak latency 664-692 ms). There was an interaction between cue, hemisphere and electrode $(F(4,88)=3.5, p=.03)$ and separate tests revealed that the size of the effect was strongest at PO7/8 $(F=30.6, p<.0005)$. At some of the electrodes, the LDAP started already between 300 and $500 \mathrm{~ms}$, as shown by an interaction between cue, hemisphere and electrode, $F(4,88)=5.2, p=.01$ (P3/4: $F=9.6, p=.005$; P07/8: $F=4.5, p=.046 ; \mathrm{P9} / 10: F=6.1, p=.02)$.

At central and frontal sites there was an ADAN between 300 and 500 ms (frontal: $F=$ 22.6, $p<.0005$; central: $F=12.6, p=.002$ ) that remained negative between 500 and 700 ms (frontal: $F=30.8, p<.0005$; central: $F=5.1, p=.03$ ) (peak latency frontal: 516-524 ms; peak latency central: 388-532). At frontal sites there was an interaction between cue, hemisphere, and electrode between 300 and $500 \mathrm{~ms}(F=6.5, p=.02)$, and between 500 and $700 \mathrm{~ms}(F=12.5, p=.002)$. Separate tests showed that the effect was stronger at F7/8 (300-500 ms: $F=22.9, p<.0005,500-700 \mathrm{~ms}: F=35.3, p<$ .0005 ) than F3/4 (300-500 ms: $F=19.4, p<.0005,500-700$ ms: $F=21.7, p<.0005$ ). At central sites the effect also differed between electrodes, as shown by an interaction between cue, hemisphere, and electrode between 300 and $500 \mathrm{~ms}(F(2,44)=8.7$, $p=.001)$, and between 500 and $700 \mathrm{~ms}(F=33.5, p<.0005)$. It was strongest at the frontally located electrodes FC5/6 (300-500 ms: $F=17.2, p<.0005$, 500-700 ms: $F=$ 21.8, $p<.0005)$.

In sum, when the uncued location was ignored and stimulus discrimination was required, a number of lateralized components were elicited by arrow cues and line cues. At posterior sites arrow cues elicited an early component between 150 and 250 ms that 
consisted of a positivity in the congruent and a negativity in the incongruent mapping. Second, at posterior sites between 300 and $500 \mathrm{~ms}$, there was a weak EDAN-like negative component in the incongruent arrow mapping, but a positive component in the congruent mapping. Between 500 and $700 \mathrm{~ms}$ there was a positive component for both arrow mappings, reflecting an LDAP. In the line task, the LDAP started already at $300 \mathrm{~ms}$ after cue onset. Perhaps, the positivity in the congruent arrow mapping between 300 and 500 ms after cue onset also reflects an early start of the LDAP. Finally, at frontal and central sites, in both arrow mappings as well as in the line task a negativity was elicited between 300 and $700 \mathrm{~ms}$, reflecting an ADAN.

\section{Discussion}

As predicted, attentional modulation of early ERP components elicited after onset of the peripheral stimulus was stronger than in the first experiment and was observed in the arrow task and the line task. In addition, after cue-onset, a number of lateralized components were elicited consistently. Similar to experiment 1 , in the arrow mappings an early component at posterior sites reversed in amplitude when the direction of the arrow reversed while the instructed direction of attention remained the same. Both this reversal and the absence of this component when attention was cued with line cues support the idea that it is related to sensory aspects of the arrows. In contrast to experiment 1 , a number of components were elicited in the arrow mappings without reversal of polarity and in the line task which suggests they might be related to shifts of attention. These were an ADAN at central and frontal sites between 300 and $700 \mathrm{~ms}$ and an LDAP at posterior sites between 500 and $700 \mathrm{~ms}$. Importantly, the LDAP already started between 300 and $500 \mathrm{~ms}$ in the line task and the congruent arrow mapping. As a result, there was a (weak) EDAN between 300 and $500 \mathrm{~ms}$ only in the incongruent arrow mapping. These results suggest a spatial and temporal overlap of the EDAN and LDAP. The LDAP might thus always have an early start, around $300 \mathrm{~ms}$ after cue onset, but perhaps is cancelled out if the EDAN is stronger, because their amplitudes are opposite. The results showed that between 500 and 700 ms LDAP was stronger in the congruent than in the incongruent mapping. The EDAN in the incongruent and the LDAP in the congruent mapping between 300 and 500 ms might similarly be a reflection of the stronger LDAP in the congruent than in the incongruent mapping.

Figure 3.8 (next page) Current source density maps of the grand averages for the congruent and the incongruent arrow mapping and the line task in Experiment 1 and Experiment 2, derived from the same across-subject lateralized averages as represented in the time plots of Fig.3.4 and Fig. 3.7 Red regions indicate positive current densities and blue regions indicate negative current densities. The electrode positions are indicated by dots and the difference between contour lines corresponds to a density change of $0.01 \mu \mathrm{V}$ per $\mathrm{cm} 2$. Maps are determined at the respective peak latencies of the maxima of the EDAN, ADAN, and the LDAP and these latencies are presented below every map. If a component was absent, maps were determined for the time window where it was expected and that window is presented below the maps. In these windows there was either (1) no significant lateralized activity at all (line task, experiment 1, window of the ADAN and LDAP), (2) no lateralized activity at the location of interest (incongruent mapping, experiment 1, window of the LDAP), or (3) significant lateralized activity at the location of interest 


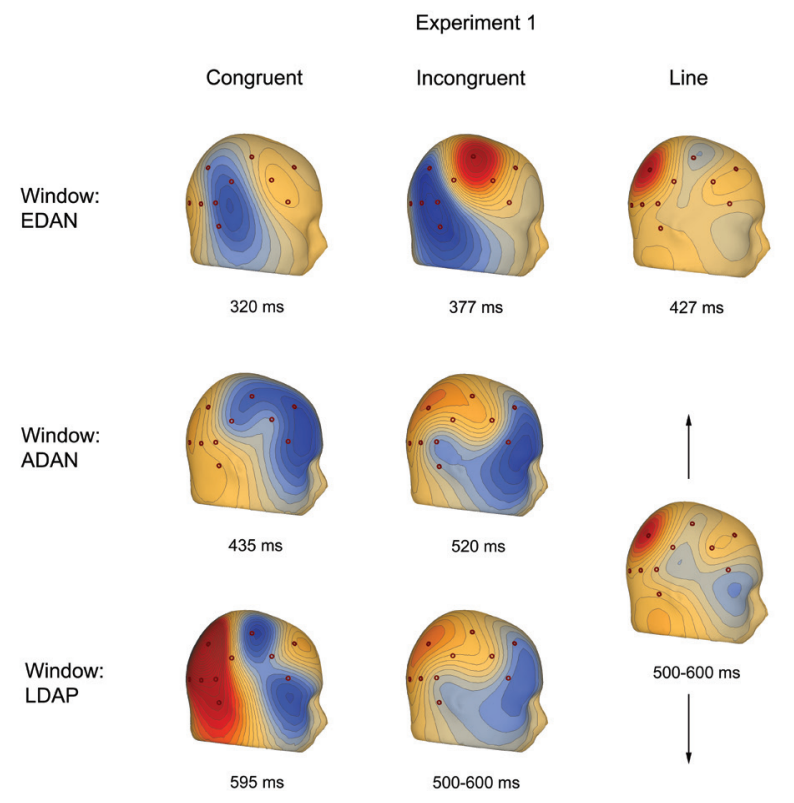

Experiment 2

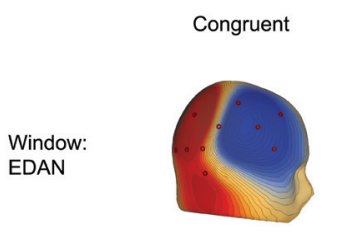

Incongruent

Line

$300-500 \mathrm{~ms}$
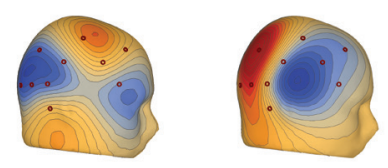

$347 \mathrm{~ms}$

$300-500 \mathrm{~ms}$
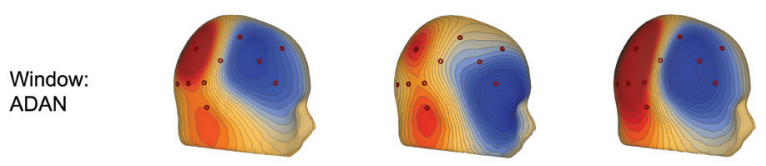

$412 \mathrm{~ms}$

$472 \mathrm{~ms}$

$521 \mathrm{~ms}$

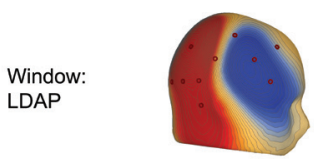

$625 \mathrm{~ms}$
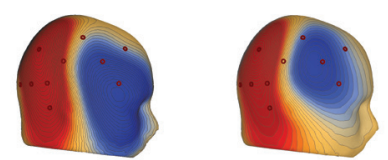

$687 \mathrm{~ms}$

$691 \mathrm{~ms}$

that differed in polarity from what was expected (congruent mapping and line task, experiment 2, window of the EDAN). In the line task of experiment 1, in the window of the EDAN, significant lateralized activity at the location of interest also differed in polarity and in addition peaked. This might be an early LDAP. 


\section{GENERAL DISCUSSION}

In two experiments the functional meaning of lateralized ERP components elicited after cue onset was studied. The goal was to discriminate components related to shifts of attention and components related to sensory aspects of the cue. For this, the physical shape of cues was controlled in two ways. In one task, the same direction of attention was indicated in two mappings by arrows pointing in opposite directions. A comparison of these mappings would show what components were related to the direction of attention and what components were related to the physical shape of the cues. In a second task, cues without any lateralized aspects were presented to exclude that lateralized activity after cue onset is elicited by shape characteristics of the cue stimulus. The two experiments differed in task instruction. In the first experiment, both validly and invalidly cued stimuli required a response ('all-go'), whereas in the second experiment responses were required only to infrequent target stimuli on valid trials ('go-nogo').

Effects of peripheral stimuli. Processing the peripheral stimulus was modulated by attention. Results of the first experiment showed RT validity effects in both arrow mappings and the line task but there was modulation of early ERP components only in the arrow task and this concerned only the P1 component. In the second experiment the task was changed and responses were required only to validly cued target stimuli. Now both the P1 and the N1 component were modulated by attention in the arrow and the line task. It has been suggested that lower attentional demands and divided attention between possible stimulus positions in the all-go detection task leads to an attenuation of early modulation (e.g. Eimer, 1994; Mangun \& Hillyard, 1991). The absence of intrinsic directional properties in line cues may have further attenuated it as there was no modulation at all in this task. An alternative perspective on the attenuation of modulation in the all-go task comes from studies in which responses were examined that people typically give when they are confronted with a sequence of binary events that occur with different probabilities and they are asked to predict on every trial which event will occur. Often participants do not use the optimal strategy of predicting the most probable event on every trial (e.g. Estes, 1964; Gal \& Baron, 1996; McCracken, Osterhout, \& Voss, 1962; Tversky \& Edwards, 1966). Instead, a matching strategy may be used that is characterized by predictions that roughly match the probability of occurrence of the two events. If participants applied a matching-strategy in the all-go experiment, with its $80 / 20 \%$ probability for valid and invalid trials, they would shift their attention to the uncued side on about $20 \%$ of the trials. On average, this would also lead to an attenuation of modulation and therefore might be an alternative explanation or an additional factor contributing to the results.

Although modulation of early ERPs after the peripheral stimulus in the first experiment was weak, robust RT validity effects were present in both arrow mappings and in the line task. This discrepancy is central to the classic discussion of early or late selection (Eimer, 1993; Eimer, 1994; Mangun \& Hillyard, 1991). Whereas Posner and colleagues proposed that RT speeding on valid trials was an effect of enhanced perceptual processing of attended stimuli (Posner, 1980; Posner, Nissen \& Ogden, 1978), others have argued that it might be the result of changes in decision bias, thus representing later processing stages (Shaw, 1984; Sperling, 1984). Early sensory ERP components are thought to reflect information processing in the visual pathways that is sensitive to the 
direction of attention (e.g. Mangun \& Hillyard, 1990). The small effects of modulation of these components in the arrow mappings and the absence of any modulation in the line task of the first experiment suggest that RT validity effects at least partly originated in later processing stages.

Cue effects. Our main goal was to discriminate components related to the physical shape of cues and components related to shifts of attention. The results of both experiments are summarized in Table 3.2. Results of both experiments showed an early positive component between 150 and $250 \mathrm{~ms}$ at posterior sites in the congruent mapping that reversed in amplitude when the physical direction of the arrow reversed while the instructed direction of attention remained the same. This reversal and the absence of a similar component in the line task suggest that it is a reflection of sensory processing of the asymmetrical arrow cues. Similar components have been reported before (Jongen, Smulders, \& van Breukelen, 2006; Van der Lubbe, Wauschkuhn, Wascher, Niehoff, Kömpf, \& Verleger, 2000; Verleger, Vollmer, Wauschkuhn, van der Lubbe, \& Wascher, 2000).

Table 3.2 Summary of lateralized ERP components in the cue-target interval, for every region, in the different task conditions, in experiment 1 and 2. Positive components are indicated by a plus-sign, negative components by a minus-sign, and the absence of lateralized activity is indicated by a zero.

\begin{tabular}{|c|c|c|c|c|c|c|c|c|c|}
\hline \multirow[b]{2}{*}{ Region } & \multirow[b]{2}{*}{ Component } & & \multicolumn{4}{|c|}{ Experiment 1} & \multicolumn{3}{|c|}{ Experiment 2} \\
\hline & & & $C$ & IC & Line & & $C$ & IC & Line \\
\hline \multirow[t]{3}{*}{ Posterior } & Early pos. & $150-250 \mathrm{~ms}$ & + & - & 0 & $150-250 \mathrm{~ms}$ & + & - & 0 \\
\hline & EDAN & $300-500 \mathrm{~ms}$ & - & - & + & $300-500 \mathrm{~ms}$ & + & - & + \\
\hline & LDAP & $500-600 \mathrm{~ms}$ & + & 0 & 0 & $500-700 \mathrm{~ms}$ & + & + & + \\
\hline \multirow[t]{2}{*}{ Frontal } & ADAN & $300-500 \mathrm{~ms}$ & - & 0 & 0 & $300-500 \mathrm{~ms}$ & - & - & - \\
\hline & ADAN & $500-600 \mathrm{~ms}$ & - & - & 0 & $500-700 \mathrm{~ms}$ & - & - & - \\
\hline \multirow[t]{2}{*}{ Central } & ADAN & $300-500 \mathrm{~ms}$ & - & 0 & 0 & $300-500 \mathrm{~ms}$ & - & - & - \\
\hline & ADAN & $500-600 \mathrm{~ms}$ & 0 & 0 & 0 & $500-700 \mathrm{~ms}$ & - & - & - \\
\hline
\end{tabular}


In the second experiment, the ADAN and the LDAP were elicited in the line task and in both arrow mappings without reversal of polarity when the direction of the arrow (but not the direction of attention) reversed. This shows that these components are independent of the physical shape of the cue and are related to the direction of attention. Interestingly, the ADAN in the incongruent mapping was elicited somewhat later than in the congruent mapping. Arrows trigger orienting in a way that is at least partly automatic (Eimer, 1997; Tipples, 2002), but in the incongruent mapping, this automatic process needs to be overruled before attention can be oriented in the correct direction. The shift of attention may therefore be delayed, and the latency shift of the ADAN may be a reflection of this delay. A delay would then also be expected when an arbitrary mapping of the direction of attention like the one in the line task is used. Figure 3.7 shows the comparison of arrow mappings and the line task in the second experiment. In comparison to the congruent mapping, the ADAN appears to be shifted in latency in the line task as well. Comparable results come from a study that showed a latency shift of the ADAN when auditory cues were used and participants were required to discriminate both the instrument and the pitch of the sound to determine cue meaning (Eimer \& Van Velzen, 2002). Together these data suggest that the ADAN is delayed whenever deriving cue meaning is more complex.

In contrast to the second experiment, in the first experiment both the ADAN and the LDAP (between 500 and $600 \mathrm{~ms}$ ) were absent in the line task and there was no LDAP and no central ADAN in the incongruent arrow mapping. Importantly, this pattern of results parallels the pattern of modulation of early sensory effects after presentation of the peripheral stimulus in the first and second experiment and suggests that the preparation of enhanced sensory processing is indicated by the ADAN and LDAP. When these components were present, there was also enhanced sensory processing for attended as compared to unattended peripheral stimuli. Thus, although stronger effects of attention after the peripheral stimulus in a go-nogo discrimination task than in an all-go detection task have been shown by others (e.g., Eimer, 1994; Mangun \& Hillyard, 1991), the present study for the first time shows similar changes in attention components after the cue. In line with this, a correlation between subject-by-subject variation in the size of the ADAN and modulatory effects of attention on the P1 component was shown in data from two go-nogo discrimination tasks (Driver, Eimer, Macaluso, \& Van Velzen, 2004). This relation between cue ERPs and modulation of ERPs after onset of the peripheral stimulus is important and might explain the absence of cue ERPs in some studies. For example, in a cross-modal cueing study, an LDAP but no ADAN was found when auditory cues were used (Green \& McDonald, 2006), whereas both components were found in another study that used similar cues (Eimer, Van Velzen, Forster, \& Driver, 2003). Interestingly, the N1 but not the early P1 was modulated in the study by Green and McDonald, whereas both components were modulated in the study by Eimer et al. This is in line then with the present results that show weak or absent modulation of cue ERPs when modulation after the peripheral stimulus was weak or absent. Finally, it was argued before that RT validity effects in experiment 1 were probably due to later processing stages, given the weak or absent modulation of components after the peripheral stimulus. The similarly weak or absent cue-related attention components in experiment 1 are in line with this conclusion. However, one limitation of the present approach is that only lateralized components were examined. In a number of studies 
nonlateralized components were demonstrated after cue onset that were also related to processes of orienting (Slagter, Kok, Mol, \& Kenemans, 2005; Slagter, Kok, Mol, Talsma, \& Kenemans, 2005; Talsma, Slagter, Nieuwenhuis, Hage, \& Kok, 2005). It cannot be excluded that they are essential to the RT modulation by attention.

The results of the first experiment showed an EDAN between 300 and $500 \mathrm{~ms}$ after cue onset in both arrow mappings and there was no reversal of amplitude. In the line task, a positive component was elicited between 300 and $500 \mathrm{~ms}$, though only at a smaller number of electrodes in the posterior region. Noticeably, at exactly the same positions the LDAP in the congruent mapping was strongest, whereas the EDAN in both arrow mappings was weaker than at other positions. This suggests that the positive component in the line task is an early LDAP. This is supported by results of the second experiment where an LDAP was elicited in the line task and in the congruent mapping between 300 and $700 \mathrm{~ms}$. As a result, there was a (weak) EDAN only in the incongruent mapping. The results of both experiments suggest temporal and spatial overlap of the EDAN and LDAP. This implies that, because their amplitudes are opposite, only the stronger one will be observed in the ERP, or in case they are equally strong no deflection will be observed. In the second experiment, the LDAP between 500 and $700 \mathrm{~ms}$ was stronger in the congruent mapping than in the incongruent mapping. The results between 300 and $500 \mathrm{~ms}$ in the arrow mappings (i.e. a weak EDAN in the incongruent mapping and an LDAP in the congruent mapping) can also be explained by a stronger LDAP in the congruent mapping.

In neither experiment there was evidence for an EDAN in the line task. This supports the claim that the EDAN is only elicited by lateralized cues (Van Velzen \& Eimer, 2003). In the arrow task, the EDAN followed the early positive component between 150 and 250 ms that reflected the processing of sensory aspects of the arrows. There was no evidence that the EDAN was related to processing the sensory aspects of arrow cues in a similar way. If it is assumed that the earlier and larger LDAP in the congruent mapping of the second experiment cancelled out the EDAN, arrow data of both experiments would contain a negativity contralateral to the direction of attention. Instead of processing sensory aspects of the cue, or the actual shift of attention independent of the physical cue, a third process is necessary to explain the EDAN. That is, the data support the idea that the EDAN is related to a component process of the direction of attention that is specific for arrow cues. Possibly, this is in line with the claim by Van Velzen and Eimer (2003) that the EDAN was an N2pc component, reflecting the filtering of irrelevant or the selection of relevant information (Luck \& Hillyard, 1994; Eimer, 1996). Although the arrows that were used in the present experiment do not require selection of a lateralized relevant part in a similar way, participants may have directed their attention more to the arrow head in the congruent mapping and more to the open end of the arrow in the incongruent mapping in order to derive the cued direction of attention. This would explain why there was no reversal of amplitude in the incongruent arrow mapping of the first experiment and suggests that the EDAN is related to cue-symbol translation processes necessary to encode directional information of arrow cues, or perhaps all types of lateralized cues.

Conclusions. In summary, by controlling the physical shape of cues this study aimed at measuring lateralized components related to shifts of attention and discriminating them from components related to sensory aspects of the cue. An early posterior 
component was related to the physical shape of the arrow cues. The ADAN and LDAP were shown to be independent of the physical shape of cues and were also elicited when nonlateralized line cues were used to direct attention. These findings support the idea that the ADAN and LDAP are related to the direction of attention. Notably, the ADAN was delayed in the incongruent arrow mapping and in the line task in comparison with the congruent arrow mapping. In addition, both the ADAN and the LDAP were smaller or even absent when modulation of early sensory effects after onset of the peripheral stimulus was diminished or absent, suggesting a relation with enhanced sensory processing after onset of the peripheral stimulus. The data also suggested temporal and spatial overlap of the LDAP and the posterior EDAN, which might explain why the latter was elicited only in some task conditions. Although the EDAN was elicited only in conditions with arrow cues and never when nonlateralized cues were used, it is unlikely that it purely reflects shape characteristics of the cue. Instead, the present data suggest that it is related to the encoding of directional information from arrow cues. 


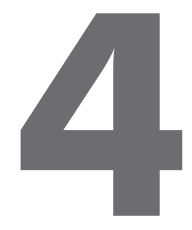

\section{Sequence effects in a spa- tial cueing task:}

\section{Endogenous orienting is sensitive to orienting in the preceding trial}

In a spatial cueing paradigm it was investigated whether endogenous orienting is sensitive to orienting processes in the previous trial. Specifically, the effect of the previous cue direction, the previous trial type (valid, invalid, neutral, catch) and target alternation effects were studied. Strategic effects were shown as attentional costs and benefits were larger after a valid than after an invalid trial. Following catch trials, an overall response slowing was observed, but costs and benefits were unaffected. This was interpreted as a reduction in alertness and as support for the dissociation between spatial and temporal attentional mechanisms. Repetition of target position per se had no effect, but in neutral trials responses were slower to targets appearing at the location that was cued in the previous trial, independent of validity of the preceding trial. This suggests that long-term inhibition-of-return can occur between trials when attention is controlled endogenously.

Jongen, E.M.M. \& Smulders, F.T.Y. (in press). Sequence effects in a spatial cueing paradigm: Endogenous orienting is sensitive to orienting in the preceding trial. Psychological Research. 
Traditionally, most reaction time (RT) studies report only average results. However, research on sequence effects has indicated that individual trials typically do not contribute to mean RT independently, because their effect is influenced by preceding trials (e.g. Bertelson, 1961; 1963; Bertelson \& Renkin, 1966; Hyman, 1953; Kirby, 1976; Kirby, 1980; McKenna \& Sharma, 2004; Soetens, 1998). Therefore, exploration of the effect of preceding events may lead to a better understanding of the way we process signals (Soetens, 1990).

Many studies of visual attention have shown that, on average, subjects respond faster to a target when they are provided with valid advance information about its location by means of a symbolic cue presented at fixation (e.g. Posner, 1980). Yet, the extant literature on this endogenous cueing paradigm does not, to our knowledge, contain a systematic study of sequence effects. The aim of the present study was to examine sequence effects in an endogenous spatial cueing task that was similar to the one used by Posner, Nissen and Ogden (1978). Cues with a predictive validity of either 50 or 80 percent were used to generate 'neutral', 'valid', and 'invalid' trials, and catch trials were added to prevent premature responses. According to Kirby (1980), sequence effects in RT tasks may originate from at least two sources. First, there may be automatic facilitation as a result of residual activity in a stimulus or response system or the bypassing of central coding processes. Second, the subject may adopt strategies that are carried out before or after arrival of the stimulus. As shown below, the literature on sequence effects in other RT and attention tasks leads to some interesting hypotheses on both automatic and strategical sequence effects in an endogenous spatial cueing task.

A study by Maylor and Hockey (1987) provides a fine example of the role of automatic processes in sequence effects in a spatial cueing paradigm. In an exogenous cueing paradigm, when the cue-target interval was $500 \mathrm{~ms}$, responses to a target at a given location were slowed when either the cue of the current trial or the target of the previous trial had been presented at that location. In a second and a third experiment where only targets were presented and the preceding four trials were included in the analysis, the results again showed slower responses when the same target location was repeated. This slowing was interpreted as a manifestation of 'inhibition of return' (IOR); the presumed tendency of attention to be inhibited to return to a location where it has just been, thus favoring novel locations (e.g. Posner \& Cohen, 1984; Posner, Rafal, Chaote, \& Vaughan, 1985; Kwak \& Egeth, 1992). Effects of IOR are likely automatic (Posner \& Cohen, 1984; Jonides, 1981). They have also been demonstrated in endogenous attention tasks, when two peripheral signals were presented in sequence and both asked for a manual response (Pratt \& Abrams, 1999; Taylor \& Klein, 2000). In our endogenous spatial cueing paradigm (Posner, Nissen and Ogden, 1978), this is comparable to the case of two target stimuli in subsequent trials. Therefore, automatic effects of peripheral onsets in trial ' $n-1$ ' might have an inhibitory effect on target detection in trial ' $n$ ' if the target is presented at the same location and if inhibitory effects survive after the attentional response to the intermediate cue. Relatively fast responses to target alternations would then be predicted.

Several others have invoked the IOR mechanism to explain sequence effects in attention tasks with informative or uninformative central or peripheral events. Although Posner and Cohen (1984) found no evidence for IOR when central predictive cues were used, it was demonstrated in a study by Rafal, Calabresi, Brennan and Sciolio (1989), 
but only when eye movements to the target location were made or planned. In a later study, Taylor and Klein (2000) also found IOR when central cues were used and eyes were kept fixated. In this paradigm, two signals were presented on each trial that were peripheral onsets or central arrows. The first was always uninformative with respect to the second. No response, a manual response or a saccadic response was made to the first and the second signal. IOR was shown when the first signal was central and required no response, and the second signal was peripheral and required a manual response. This build-up of a trial has similarities with a standard endogenous cueing task (Posner, Nissen, \& Ogden, 1978), but an important difference is that in Taylor and Klein's study, the first signal was always uninformative with respect to the second. This was done to prevent effects of voluntary attention. As stated by Taylor and Klein, previous reports with central arrow cues may have failed to observe IOR because inhibitory effects of central arrow cues were "overpowered" by facilitatory effects of voluntary orienting. Since our arrow cues are valid $80 \%$ of the time, we do not expect an effect of IOR within trials for the same reason. However, studies by Maylor and Hockey (1987) and Pratt and Abrams (1999) have shown that effects of IOR can carry over to the next trial. While a cue will be predictive concerning the target location in that particular trial, it will not be predictive with regard to the next trial. Therefore, central cues that direct attention to the left or to the right in one trial might have an inhibitory effect on attentional orienting to the same location in the next trial.

In summary, as an automatic effect, a target alternation effect may be observed, based on various studies that used peripheral stimuli (Maylor \& Hockey, 1987; Pratt \& Abrams, 1999; Taylor \& Klein, 2000). Furthermore, an analysis of sequence effects is apt to investigate whether central endogenous cues lead to IOR, because the cue on trial ' $n-1$ ' has lost all its predictive power after the target in that trial has elapsed, possibly leaving traces of inhibition of return that can be measured on trial ' $n$ ' because they are not overpowered anymore by voluntary orienting in trial ' $n-1$ '. Add to this other indications that IOR can occur between trials (Maylor \& Hockey, 1987; Pratt \& Abrams, 1999), and the hypothesis ensues that central cues have an inhibitory effect on attentional orienting to the same location in the next trial. Both effects of orienting in trial ' $n-1$ ' would be visible in pure form when the cue in trial ' $n$ ' is neutral, and could simply add up to the effect of the cue in trial ' $n$ ' if it is directional.

A role for strategic adjustments in sequence effects has been demonstrated in focussed attention or conflict tasks. Gratton et al. (1992, exp. 1) used a noise-compatibility paradigm (Eriksen \& Eriksen, 1974), and studied changes from trial to trial in the participants' sensitivity to irrelevant noise letters surrounding a central target. Sequence analyses showed a larger interference effect on trials that followed a trial with compatible in comparison to incompatible noise. This effect was explained by supposing an expectation that was created by the type of noise on the previous trial, effectively an expectation for repetitions. Similarly, in a Simon task, it has repeatedly been shown that if the Simon effect is analyzed as a function of the spatial stimulus-response correspondence in the preceding trial, a sizeable effect is only present after corresponding trials (e.g. Ridderinkhof, 2002; Stürmer, Leuthold, Soetens, Schröter, \& Sommer, 2002). In the current study, four types of trials (valid, invalid, neutral, catch) were presented. If momentary strategical adjustments in the endogenous cueing paradigm are similar to those in the Eriksen noise-compatibility and Simon task, a valid trial might enhance 
the expectation that it is beneficial to direct attention to the cued location whereas an invalid trial might weaken this expectation or even promote orienting to the uncued location. This would lead to an increase of both costs (the difference between invalid and neutral) and benefits (the difference between valid and neutral) of attention after a valid trial in comparison with after an invalid trial.

To summarize, this study aimed at examining sequence effects in an endogenous spatial cueing paradigm to find out if, in addition to the current cue, other recent events affect attentional orienting in a trial. The effects of interest are illustrated in Figure 4.1. First, strategical adjustments will be studied by examining the interaction between validity of the preceding trial and validity of the current trial to find out if costs and benefits depend on validity of the preceding trial. In addition, it will be examined if similar to other studies (Alegria, 1978; Correa, Lupiáñez, Milliken, \& Tudela, 2004; Los, 2004) an overall delay in RT is present after a catch trial. Second, automatic effects of the previous cue and target will be examined to find out if there is support for (a) an advantage for target alternations in comparison to target repetitions, and (b) effects of IOR after central endogenous cues that "survive" until the target in the next trial. The relation between preceding cue and current target can be described in terms of validity, and because it occurs between trials it will be referred to as inter-trial validity'. The hypothesis then amounts to slower responses if the preceding trial's cue and current trial's target are corresponding, or 'inter-trial valid', than when they are non-

TRIAL N-1

TRIAL N

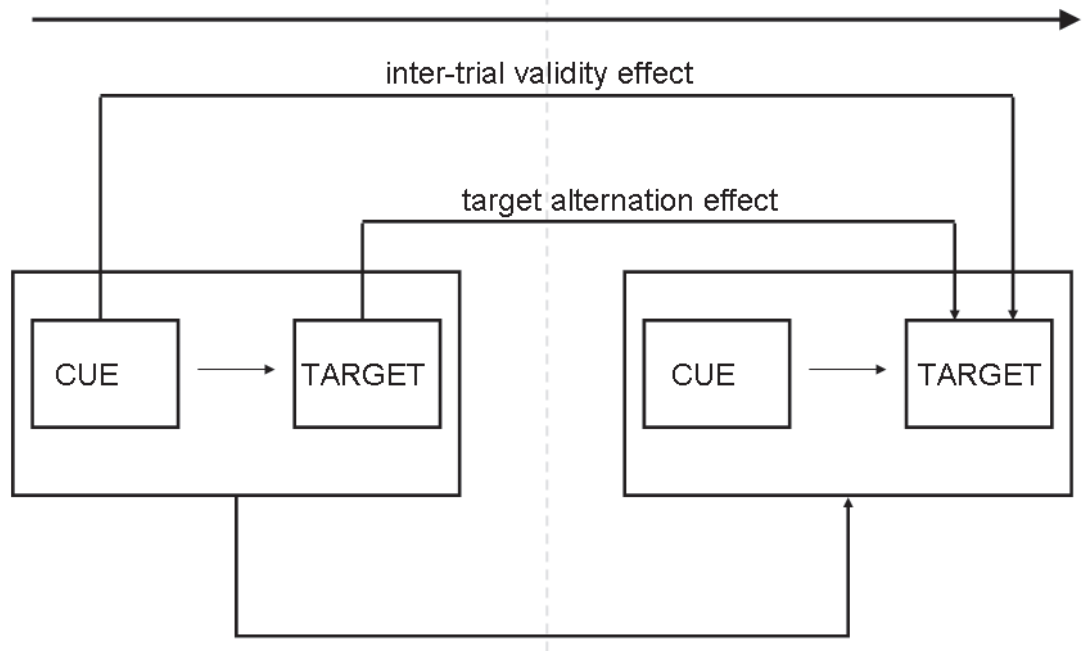

validity preceding trial $\times$ validity current trial

Figure 4.1. Sequence effects of interest are illustrated in a two-trial sequence (previous trial is indicated as: trial n-1; current trial is indicated as: trial n). First of all, the dependence of attention effects on validity of the preceding trial is illustrated by the interaction between validity of the preceding and current trial. Second, effects of target alternation and repetition will be examined. Finally, inhibitory effects of a cue on the target in the next trial will be examined. The relation between preceding cue and current target can be described in terms of 'inter-trial validity'. 
corresponding, or 'inter-trial invalid'. The same hypotheses were already examined in an unpublished pilot study from our lab. The results showed weak (trend-level) support for strategical adjustments, as costs were smaller after an invalid than after a valid or neutral trial. After catch trials, there was a substantial overall delay in responses, but costs and benefits of attention were unchanged. There was no advantage for target alternations in successive trials, but central endogenous cues had an inhibitory effect on attentional orienting to the same location in the next trial. This effect was remarkably independent of the validity of both the previous and the current trial, and it combined with the effect of the present cue in a purely additive way. In this previous study the order of trial types was truly random with the disadvantage that the number of occurrences of rare sequences was uncontrolled and thus low in some participants. The current experiment aimed at replicating these results, using more trials and a complete balancing of all possible sequences.

\section{Method}

\section{Subjects}

Eighteen subjects (4 male), 21-30 years old (mean age 25), participated in the study. All had (corrected to) normal vision and four were left-handed. The experiment received prior approval of the institutional ethics committee. All participants signed an informed consent and were paid for their participation. Data of two subjects were excluded from the analyses, because the number of errors and outliers was very high. ${ }^{1}$ Data of the remaining sixteen subjects will be described.

\section{Stimuli, apparatus and procedure}

Subjects were comfortably seated in a dimly lit, sound attenuated room. The stimuli were presented on a VGA monitor at $75 \mathrm{~cm}$. To control stimulus presentation, record accuracy and latency, ERTSVIPL V3.32c (Beringer, 1987) was used. Target stimuli consisted of dark gray-and-light gray, vertical square-wave grating stimuli $\left(2.1^{\circ}, 7\right.$ cycles/degree) presented to the left or right $\left(7^{\circ}\right)$ of fixation on a gray, equiluminant background. The task was a detection task where every grating stimulus required a right index finger response, independent of its spatial frequency or location. The response was given on a microswitch which was covered by a round response button $1.5 \mathrm{~cm}$ in diameter that was placed centrally in front of participants.Catch trials were added to prevent premature responses.

Each trial started with a fixation cross, which stayed on throughout the trial (see Figure 4.2). After $100 \mathrm{~ms}$, a cue was presented for $600 \mathrm{~ms}$ that consisted of an arrow to the left $(<<)$ or to the right $(>>)$ both with a predictive validity of $80 \%$, or a neutral cue $(<>)$ that had no predictive validity $(50 \%)$, meaning that the target could appear on either location with equal likelihood. At $900 \mathrm{~ms}$ after cue onset, the target was presented for $200 \mathrm{~ms}$. Then there was a $2000 \mathrm{~ms}$ inter-trial interval.

The main task consisted of 2349 trials. Trials were presented in random order with the restriction that every trial type was immediately preceded by every trial type a fixed

1 The pattern of results was the same when these subjects were included, but effects were stronger without them. 
number of times in the same proportion as the overall proportion. The proportion of valid : invalid : neutral : catch trials in the experimental block was 8:2:4:3. Since every trial was a combination of the factors cue (3: left, neutral, right) and target (3: left, right, catch), there were $3 \times 3=9$ trial types, and $9 \times 9=81$ different sequences. The number of trials for the rarest sequences (e.g. 'invalid cue left' preceded by an 'invalid cue left') was 8; for all other sequences it was a multiple of 8.

Subjects were instructed to respond quickly and accurately and to maintain central eye fixation during the trials. Corrective feedback (an error message and short tone) was given on misses and false alarms and on responses that preceded target onset, were too fast $(<120 \mathrm{~ms})$ or too slow $(>800 \mathrm{~ms})$. After three 12-trial practice blocks, and feedback about their reaction time after every block, subjects performed the main task. They received feedback about their reaction time and could pause for a moment every 64 trials. After each pause, the last trial prior to the pause was repeated in order to create the proper "history" for the next trial, and it was later omitted from the analyses so that our sequence effects were not confounded by the pauses, and sequences remained perfectly balanced. The very first trial was deleted for the same reason, leaving 2312 trials for analyses.

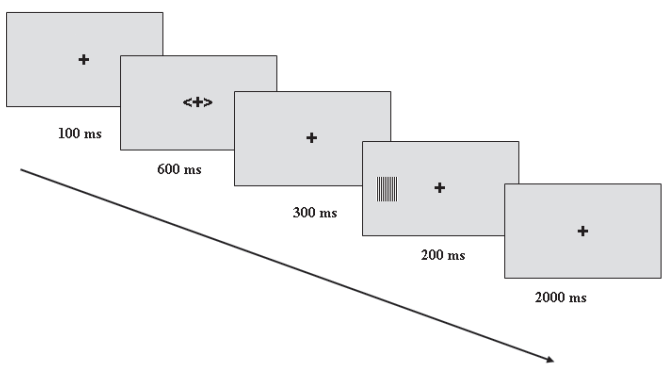

Figure 4.2. An example of a trial sequence in which a neutral cue is followed by a target to the left.

\section{Data analyses}

Trials that contained an error or that followed an error were deleted from the analyses. Possible errors were false alarms, misses, responses on the cue, and responses on the target that were either faster than $120 \mathrm{~ms}$ or slower than $800 \mathrm{~ms}$. Main analyses of validity and sequence analyses were done. It was examined (a) if costs and benefits of spatial attention in trial ' $n$ ' were modified by validity of trial ' $n-1$ ', and if a catch trial ' $n-1$ ' caused an overall slowing of RT in trial ' $n$ ', and (b) if cue direction and target position of trial ' $n-1$ ' had an influence on attention in trial ' $n$ '. The latter effects were first examined when trial ' $n$ ' was neutral and then when trial ' $n$ ' had a directional cue.

\section{Results}

An alpha level of .05 was maintained for all statistical tests. 


\section{Errors and outliers}

The average percentage of RT-outliers ranged from 0 to 2.6 (average 0.79 ). The percentage of false alarms ranged from 0.25 to 7.35 (average 3.6), of misses from 0 to 0.79 (average 0.22 ), and of responses on the cue from 0.09 to 1.17 (average 0.49 ). After exclusion of errors and outliers, $98.0 \%$ of the data remained for analyses.

\section{Validity}

A 3 (validity: valid, neutral, invalid) $\times 2$ (target position: left, right) ANOVA was used to analyze effects of validity disregarding sequence effects. Figure 4.3 displays reaction times as a function of the relevant task variables. Attentional orienting effects were reflected by a main effect of validity, $F(2,14)=48.4, \quad p<.0005$. Post-hoc analyses showed both significant costs (invalid vs. neutral), $F(1,15)=58.8, p<.0005$, and benefits (valid vs. neutral), $F(1,15)=85.0, p<.0005$. An interaction between validity and target position, $F(2,14)=5.2, p=.02$, indicated larger benefits for target left than for target right, $F(1,15)=11.0, p=.005$. Taken together, these results confirmed that participants succesfully used the arrow cues to orient their attention.

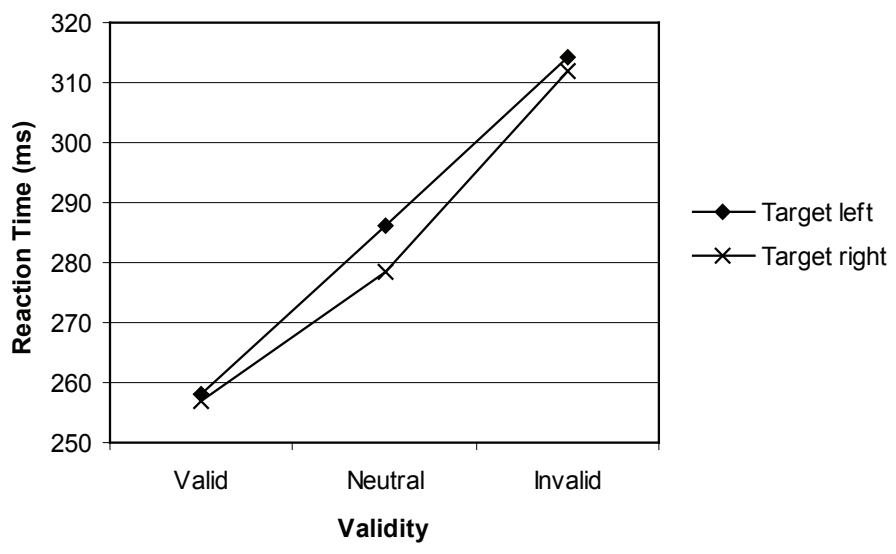

Figure 4.3. Effects of validity and target location on mean reaction time.

\section{Sequence effects}

Costs and benefits: validity of the preceding trial. A 4 (validity of the preceding trial(n-1): valid, invalid, neutral, catch) $\times 3$ (validity of the current trial $(\mathrm{n})$ : valid, invalid, neutral) ANOVA was performed to examine the influence of validity of the previous trial on costs and benefits of the current trial. There were main effects of validity of the current trial, $F(2,14)=50.9, p<.0005$, and preceding trial, $F(3,13)=6.4$, $p=.007$, and there was an interaction between these factors, $F(6,10)=3.8, p=.031$. Results are displayed in Figure 4.4a and 4.4b. They suggest the hypothesized delay after a catch trial and larger costs and benefits after a valid than after an invalid trial.

To verify if after a catch trial RTs were delayed, a reverse Helmert contrast was used to compare catch trials with the average of the other three preceding trial types in an analysis with the same factors as before. It confirmed that after a catch trial RTs were slower than after the average of the other three trial types, $F(1,15)=17.9$, 
$p=.001$. Post-hoc analyses showed that in comparison with the average of the other three preceding trial types there was no difference in costs or benefits (both $F(1,15)$ $<1$ ) after a catch trial. Figure 4.4b shows that as hypothesized costs and benefits were smaller after an invalid trial than after a valid trial. To verify this, a 3 (validity of the preceding trial(n-1): valid, invalid, neutral) $\times 3$ (validity of the current trial $(\mathrm{n})$ : valid, invalid, neutral) ANOVA was performed. In addition to the main effects, there was an interaction between validity of the preceding and current trial, $F(4,12)=5.7, p=.008$. Posthoc-analyses showed both smaller benefits $(F(1,15)=6.7, p=.02)$ and costs $(F(1,15)=23.1, p<.0005)$ when the preceding trial was an invalid trial than a valid trial. Costs after an invalid trial also were significantly smaller than after a neutral $(F(1$, 15) $=19.1, p=.001$ ) trial.

a

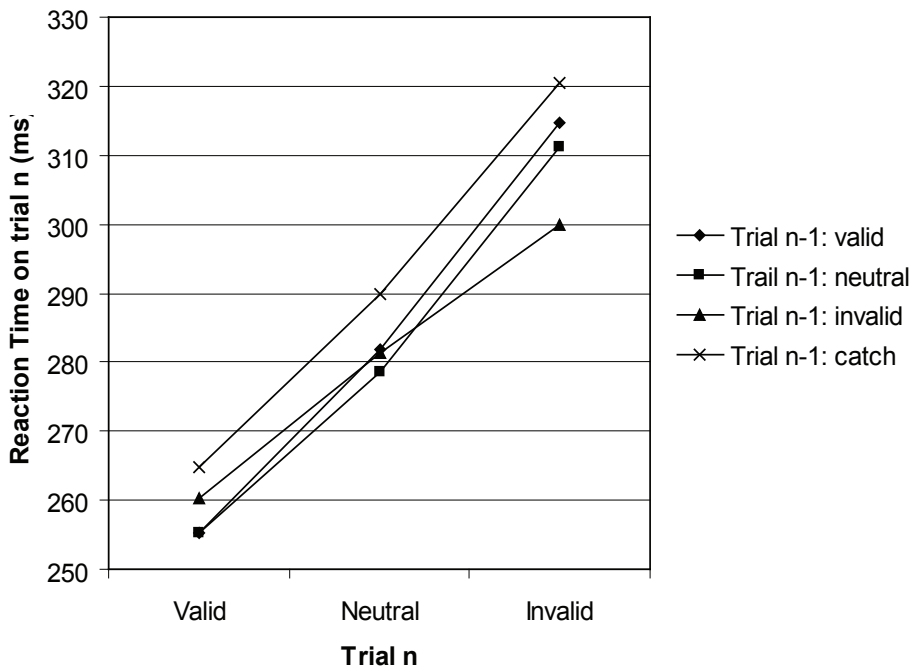

b

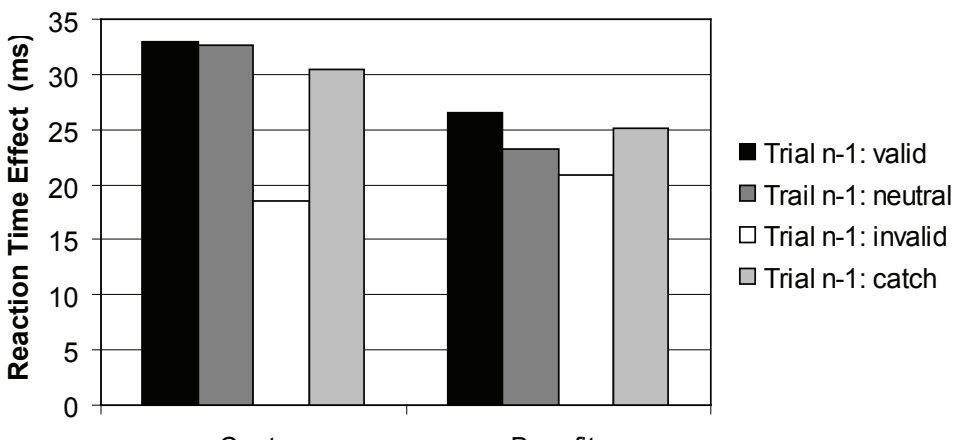

Costs

Benefits

Trial $n$

Figure 4.4. (a) Mean reaction times in valid, invalid and neutral 'current' trials and (b) costs and benefits in the 'current' trial (current trial indicated as: trial $n$ ), both as a function of the type of preceding trial (indicated as: trial $n-1$ ). 
To summarize, there was an overall slowing of responses after a catch trial but no change in costs or benefits. In addition, costs and benefits were larger after a valid than after an invalid trial, matching our hypotheses on strategic effects.

Effects of the preceding cue and target. The influence of preceding cue direction and target position were first examined in circumstances where the present cue did not direct attention: neutral trials. A 3 (cue direction of the preceding trial: left, neutral, right) $\times 2$ (target position of the preceding trial: left, right) $\times 2$ (target position of the current trial: left, right) ANOVA was carried out on the mean RTs of neutral trials. There were main effects of the previous $(F 1,15)=9.1, p=.01)$, and the current $(F(1,15)=6.4, p=.02)$ target. Subjects were faster when the previous target was presented to the left or the current target was presented to the right. The interaction between preceding and current target that reflected the effect of target alternation was not significant, $F<1$. Instead, there was an interaction between previous cue direction and current target position, $F(2,14)=5.7, p=.02$. If the data are reordered, this interaction can be described in terms of inter-trial validity (Figure 4.1). Inter-trial valid and inter-trial invalid trials can be compared to test our hypothesis that responses are faster on inter-trial invalid trials, and inter-trial neutral trials can serve as a baseline to find out if any differences between inter-trial valid and inter-trial invalid trials are due to inhibition or facilitation. The results are displayed in Figure 4.5. A 3 (inter-trial validity: valid, neutral, invalid) $\times 2$ (target position of the preceding trial: left, right) $\times$ 2 (target position in the current trial: left, right) ANOVA showed a main effect of intertrial validity, $F(2,14)=6.5, p=.01$. Posthoc-analyses showed slower responses to inter-trial valid than to inter-trial invalid trials, $F(1,15)=11.4, p=.004$, supporting our hypothesis. Responses to inter-trial valid responses were also slower than to intertrial neutral sequences, $F(1,15)=9.6, p=.007$, but inter-trial invalid and inter-trial neutral responses did not differ, $F<1$. In sum, the results showed an inhibitory effect

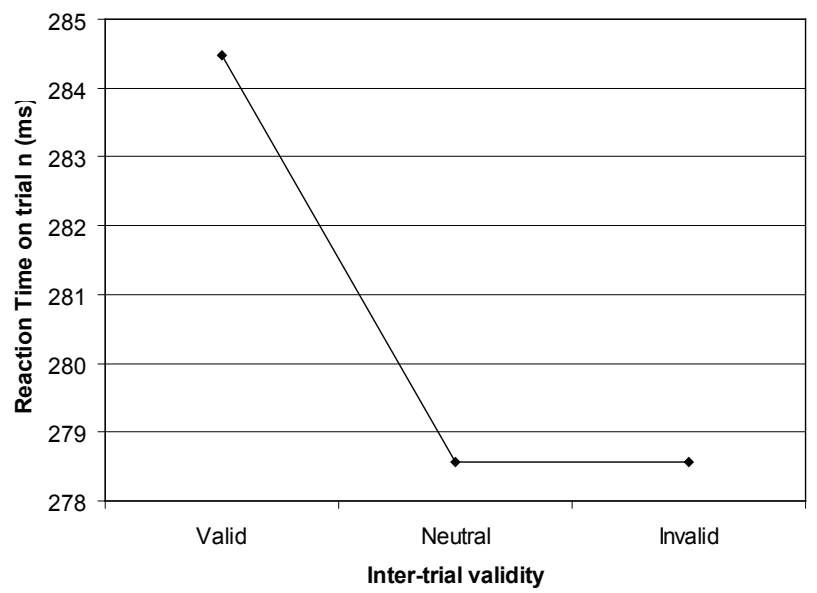

Figure 4.5. Sequence effects of previous cue and target on mean RTs in neutral trials. Inter-trial validity refers to the relation between previous cue direction and current target position and is neutral if the previous cue was neutral, is valid if previous cue direction and current target position are the same, and is invalid if previous cue direction and current target position differ. 
for the position where attention was directed to by the cue in the preceding trial and this effect was independent of the target position and cue validity in the preceding trial. There was no evidence for an effect of target alternation.

Second, the effect of preceding cue direction and target position was examined for left and right-cued trials. A 3 (cue direction of the preceding trial: left, neutral, right) $\times 2$ (target position of the preceding trial: left, right) $\times 2$ (cue direction of the current trial: left, right) $\times 2$ (target position of the current trial: left, right) ANOVA was conducted on the mean RTs. There was no effect of target alternation as shown by an absence of interaction between previous and current target, $F=1$. In addition there was no effect of the previous cue on the current target, $F<1$. There was an interaction between previous cue and previous target, $F(2,14)=8.7, p=.003$, and between current cue and current target, $F(1,15)=92.2, p<.0005$. These are effectively validity effects of the previous and current trial and have been described above. In addition, the four-way interaction was significant, $F(2,14)=10.8, p=.001$. This interaction is part of the interaction that was already described as the effect of validity of the previous trial on validity of the current trial (cf. Figure 4.4). In sum, in trials where attention was driven by a directional cue there was no evidence for inhibitory effects of the previous cue that were shown in neutral trials. Similar to neutral trials there was no effect of target alternation. Instead, strategic effects were shown.

\section{Discussion}

Sequence effects in a spatial endogenous cueing paradigm were explored with separate analyses of the effect of cue validity on costs and benefits in the next trial and of effects of the previous cue and target.

Both costs and benefits of attentional orienting were smaller after an invalid than after a valid trial. The typical explanation is that subjects have some degree of strategic control and adapt their utilisation of the cue depending on if it correctly or wrongly directed their attention to a location on the previous trial. Costs and benefits have been described in terms of mental processes involving engagement, disengagement and movement of attention. As they are both affected after an invalid trial, it could either mean that attention is not oriented on a number of trials, or is not fully engaged to the indicated location. Comparable strategic effects have been demonstrated in focussed attention and conflict paradigms (Gratton et al., 1992; Ridderinkhof, 2002; Stürmer et al., 2002). Although these effects have typically been attributed to strategic control, Hommel and colleagues proposed a "feature integration account" that does not require voluntary control (Hommel, Proctor, \& Vu, 2004; Hommel, 2004). It assumes that co-occurrence of a cue and target in a trial leads to a representation of the relation in which their features are integrated. This relation would be reactivated in the next trial, and would influence performance in such a way that good performance is predicted if validity is repeated but interference would occur if it alternates. This boils down to the same prediction of smaller benefits and costs after an invalid trial than after a valid trial.

There was an overall delay after a catch trial. It has been reported that a decrease of the probability of stimulus occurrence causes an increase of RTs (Gordon, 1967; Näätänen, 1972). Snodgrass (1969) attributed this to a decrease in the frequency of anticipations, but Alegria (1978) analyzed trial sequences and showed that independent 
of the overall catch-trial probability, after an uninterrupted sequence of targets, RT became as fast as when catch-trial probability was zero. From this, he concluded that event probability effects boiled down to sequence effects. In two recent cueing studies, sequence analyses also showed an increase in RTs in trials that followed on catch trials (Correa, Lupiáñez, Milliken, \& Tudela, 2004; Los, 2004). In agreement with Alegria (1978), Correa et al. suggested that the increase in RT was related to a decrease in preparation after a catch trial. Alternatively, Los proposed that it is caused by processes of inhibition that follow the presentation of a cue to prevent premature processing.

The combination of a decrease in speed but unchanged costs and benefits following a catch trial supports the distinction between orienting and alerting processes of attention. Similarly, Fernandez-Duque and Posner (1997) showed a decrease in speed but no change of the validity effect in an exogenous cueing paradigm when an auditory alerting cue was presented. Separate anatomical networks have been proposed for these processes of attention (Posner and Peterson, 1990). Coull and Nobre (1998) showed that, analogous to spatial orienting, attention can be "temporally oriented" to a point in time and together, temporal orienting and alerting processes were suggested to be temporal processes of attention. Correa et al. (2004) demonstrated that the sequence effect of catch trials on RTs was independent of the validity effect of temporal orienting. Likewise, our results showed that the effect of catch trials did not interact with the effects of spatial orienting. All together, these results therefore support the notion of different spatial and temporal processes of attention that can act independently (Coull \& Nobre, 1998; Griffin, Miniussi, \& Nobre, 2002; Los, 2004; Milliken, Lupiáñez, Roberts, \& Stevanovski, 2003). Catch trials mainly appear to affect processes of alertness, independently from other mechanisms of attention.

Another effect that was examined was an advantage for target alternations that has been attributed to exogenous IOR (Pratt \&Abrams, 1999; Maylor \& Hockey, 1987; Taylor \& Klein, 2000). At least for neutral-neutral sequences, feature integration (Hommel et al., 2004) might have predicted the opposite (an advantage for target repetition), because that would represent repetition of all features. However, no advantage was found for target alternations or repetitions in successive trials. A sufficient explanation is probably that the time between targets in consecutive trials was rather long (about four seconds), thereby reducing short-lasting exogenous IOR effects, although Tipper, Grison and Kessler (2003), using a different paradigm, found effects of IOR that lasted for even 13 minutes. Another reason may be that we used a simple detection task, whereas in other studies responses were based on location (Taylor \& Klein, 2000) or identity (Pratt \& Abrams, 1999) of the stimulus, possibly enhancing exogenous orienting effects because the target and its location are more important in these paradigms. In Maylor and Hockey (1987), no predictive central cues were used that could interfere with traces of exogenous orienting.

In addition to target-target effects we examined effects of the endogenous cue that may carry over to the next trial, when it has lost its predictive power. To describe the relation between previous cue and current target the term "inter-trial validity" was introduced. In inter-trial valid trials the previous cue direction and current target location were similar, whereas they were different in inter-trial invalid trials. In inter-trial neutral trials the previous cue was neutral and these trials served as a baseline. Endogenous IOR would be manifested by faster responses on inter-trial invalid than inter-trial valid 
trials. This effect was demonstrated in "current" neutral trials. A comparison with intertrial neutral trials and an analysis of inter-trial costs and benefits showed that the effect was due to inhibition of the position that was cued in the previous trial. This effect was independent of validity in the previous trial. In the pilot study that we mentioned in the introduction the inhibitory effect of the previous cue was present also in trials with directional cues where it combined with the validity effect of the present trial in an additive way. However, in the current study strategic effects in trials with directional cues were stronger than in the pilot experiment maybe as a consequence of the substantial increase in the number of trials and task duration that was needed to achieve balancing of the sequences. These strategies may have had an overpowering effect on the automatic inhibitory effects of the previous cue. A comparison of the two experiments showed that the "current" effect of validity was larger now. Therefore, inhibitory effects of the previous cue might also have been overpowered by facilitatory effects of voluntary orienting in the current trial.

In sum, the results show that analyses of sequence effects can enhance our insights in processes that influence task performance. First, after a catch trial overall slower responses but intact costs and benefits were shown, supporting the independence of attentional mechanisms of orienting and alertness. Second, strong strategic effects were demonstrated in trials with directional cues where costs and benefits depended on validity of the previous trial. Third, in neutral trials orienting was influenced by the direction of the cue in the previous trial. More specifically, the position that was cued in the previous trials was inhibited. We therefore propose that endogenous shifts of attention result in long-term inhibitory processes that are independent of exogenous effects and validity of the previous trial. This independence is remarkable because the attentional response to the previous target is closer in time than the response to the previous cue. It would be interesting to study similar effects in a task that requires closer examination of the target. These inhibitory processes might also play a role in the 'preference of attention to switch' that was suggested by Posner (1980) when no benefits of attention were found in a cueing paradigm where attention was cued for a whole block instead of on each trial (Posner, Snyder, \& Davidson, 1980). 


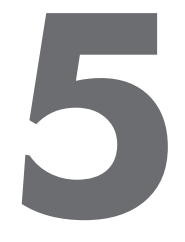

\title{
Attentional bias and general orienting processes in bipolar disorder
}

\begin{abstract}
Attentional biases for emotional stimuli and general orienting processes were examined in bipolar disorder, using a modified dot-probe task with a spatial cueing paradigm incorporated in it. Bipolar patients in a euthymic state (i.e. remission), bipolar patients in a mildly depressed state, and nonpsychiatric controls participated. General orienting results showed that within the patient group as a whole, measures of depressed mood were positively associated with a relative inability to disengage attention. Attentional bias results showed that bipolar patients in a mildly depressed state, in comparison with controls, directed their attention away from depression-related words and positive words. The bias away from positive words was equally present in both patient groups and part of a trait effect, demonstrated by the comparison of patients in a euthymic state and controls. The bias away from depression-related words was mood statedependent and within the patient group as a whole correlated negatively with measures of depressed mood. It is proposed that biases for emotional stimuli are related to the transition of mood states, characteristic for bipolar disorder.
\end{abstract}

Jongen, E.M.M., Smulders, F.T.Y., Ranson, S.M.G., Arts, B.M.G., \& Krabbendam, L. (in press). Attentional bias and general orienting processes in bipolar disorder. Journal of Behavioral Therapy and Experimental Psychiatry. 
According to cognitive models (e.g., Beck, 1976; Bower, 1981) mood-congruent biases play a causal and maintaining role in depression. In contrast to the large number of studies on unipolar depression and dysphoria (for reviews, see Mathews \& MacLeod, 2005; Mogg \& Bradley, 2005), research on cognitive biases in bipolar depression is scarce. Although both unipolar depression and bipolar disorder are characterised by episodes of remission and depression, in bipolar disorder episodes of mania and mixed episodes also occur (Diagnostic and Statistical Manual of Mental Disorders; 4th ed. (DSM-IV); American Psychiatric Association, 1994). Transitions between these episodes go together with changes in mood and cognitive functioning (Cuellar, Johnson, \& Winters, 2005; Murphy \& Sahakian, 2001). Given these differences between the disorders, presupposing similarity of unipolar and bipolar depression is probably too simplistic. The role of cognitive biases should therefore also be studied in bipolar depression. In only one study, an attentional bias for depression-related but not euphoria-related words was shown in a group of bipolar depressed patients (Lyon, Startup, \& Bentall, 1999) on an Emotional Stroop task (Williams, Mathews, \& MacLeod, 1996). To study whether attention is directed towards or away from emotional stimuli, the dot-probe task (MacLeod, Mathews, \& Tata, 1986) is preferred. Typically, in a dot-probe task two words (or pictures) of different emotional valence (e.g. one neutral and one depressionrelated) are presented together, side by side. Immediately following the word-pair, a probe appears at one of the positions previously occupied by a word. Participants signal the presentation of the probe by pressing a key, and their locus of attention is indicated by relatively fast responses to probes that appear at an attended location. Faster responses to probes at the previous location of a depression-related word thus would indicate an attentional bias toward depression-related words. The dot-probe task has not been applied in bipolar disorder.

In addition to mood-related attentional biases, the capacity to control attentional orienting in general may also play a role in negative affect (Derryberry \& Rothbart, 1988). The process of orienting involves sub-processes of attentional shifting, engagement and disengagement (e.g. Posner \& Peterson, 1990). In a nonclinical sample, results from questionnaire-data showed an inverse relation between the ability to shift attention and negative affect (Derryberry \& Rothbart, 1988). Similarly, those participants from a nonclinical sample who had difficulty to disengage their attention on an attentional orienting task showed the largest increase in negative affect in response to a distressing film (Compton, 2000). A similar relation between (sub-processes of) orienting and measures of negative mood may be present in a clinical sample of patients with bipolar disorder.

The aims of the present study were twofold. First, processes of attentional bias for emotional stimuli were investigated in bipolar disorder using a modified dot-probe task. Second, general orienting processes in bipolar disorder were examined. For this purpose, a spatial cueing paradigm (e.g., Posner, Nissen, \& Ogden, 1978), a classic measure of attentional orienting ability, was used and incorporated in the dot probe task.

Given the episodic nature of bipolar disorder, any bias or impairment in cognition may be either a state characteristic, that waxes and wanes with the mood episodes, or a trait characteristic, that is present regardless of the level of psychopathology. A sample of patients with bipolar disorder was tested and divided into one group that was in a mildly depressed state and another group that was in a euthymic state (i.e. remission). 
A healthy control participant was matched individually to each patient. Three comparisons were of interest. First, patients in the mildly depressed state were compared to a matched control-group. Any differences reflect the combination of trait and state effects. Trait and state effects were examined separately in two additional analyses. Trait effects were reflected by the difference between patients in a euthymic state and matched controls. State effects were reflected by effects of level of depression within the patient-group as a whole. These three analyses were conducted for the spatial cueing part of the task and were repeated to study attentional bias for emotional words.

General orienting capacity was examined in patients with bipolar disorder and control participants. The abilities to disengage and shift attention were both expected to be inversely related to negative mood. Regarding attentional bias, positive words were included in the dot-probe task in addition to depression-related words, to control for the possibility of a general emotionality effect that would be indicated by similar biases for positive and depression-related words (cf. Martin, Williams, \& Clark, 1991). A bias toward depression-related words was hypothesized in the patient group that was in a mildly depressed state as it has been demonstrated in unipolar depression and dysphoria (for reviews, see Mathews \& MacLeod, 2005; Mogg \& Bradley, 2005). Healthy participants might show a 'protective' bias, directing their attention away from negative stimuli (e.g., McCabe \& Toman, 2000). A bias away from positive words was hypothesized in the patient group that was in the mildly depressed state, as it has been shown for happy faces in dysphoria (e.g., Bradley et al., 1998). Healthy participants on the other hand were hypothesized to preferentially process positive stimuli (Gotlib, McLachlan, \& Katz, 1988; Koster et al., 2005). For both wordtypes, patients in a euthymic state might either display a similar bias as the mildly depressed patients, indicating a trait-like vulnerability (for a review, see Gotlib \& Krasnoperova, 1998), or they might be more similar to the healthy controls, indicating that the biases depend on current mood state.

\section{Method}

\section{Participants}

The study was approved by the ethics committee in the local academic hospital. All participants gave informed consent and received 25 euro for participation.

Patients were outpatients (plus one inpatient) recruited through the psychiatry department of the local academic hospital. Every participating patient was diagnosed with bipolar disorder. Two patients had a comorbid disorder of panic disorder with agoraphobia. The Operational Criteria Checklist (OPCRIT; McGuffin, Farmer, \& Harvey, 1991) had been used by a psychiatrist affiliated to the hospital to determine diagnoses according to the DSM-IV (1994). Severity of depression and mania were assessed with the Hamilton Rating Scale for Depression (Hamilton, 1960) and the Young Mania Rating Scale (Young et al., 1978) respectively. All patients received mood stabilizing medication (predominantly lithium or valproaat, and 2 were taking lamotrigine), 6 received antipsychotics (olanzapine or risperidon), and 5 received antidepressants (paroxetine, fluoxetine, venlafaxine, or citalopram).

Participants in the healthy control-group were recruited through a newspaper-advertisement. Based on self-report through a phone screen, they were excluded if they 
had a psychiatric or neurological history or if first-degree relatives had a psychiatric history. In addition, they were excluded if they scored ten or more on the Dutch version of the Beck Depression Inventory (BDI; Beck et al., 1961; Kendall et al., 1987) in the lab. Each control participant was matched individually to a patient for age, handedness, sex, and IQ (measured with a shortened version of the Groninger Test of Intelligence (GIT; Luteijn \& van der Ploeg, 1983)).

The final sample of participants consisted of 29 patients and 29 controls. All had (corrected to) normal vision and their first language was Dutch. The patients were divided into a group that was in a mildly depressed state ( $n=16,9$ men, all right-handed) and a group that was in a euthymic state $(n=13,6$ men, 1 left-handed) based on the median score (11) on the BDI. Due to the individual matching procedure, the controlgroup was also divided in a group matched to the group in a mildly depressed state, and a group matched to the group in a euthymic state. None of the patients showed evidence of becoming manic or having a mixed episode at the time of testing, as confirmed by the scores on the YMRS (mean 1.3).

\section{Task description}

The task that was used in the present study was a combination of a dot-probe task and a spatial cueing paradigm. In Figure 5.1a, this is demonstrated with an example of a trial, and timing definitions are presented.

Similar to a standard dot-probe task, a word pair was presented on every trial, with one word left and one word right from fixation. Emotional-neutral word pairs consisted of a depression-related or a positive word paired with a neutral word, matched for word length and word frequency (CELEX, Baaijen, Piepenbrock, \& Gulikers, 1995). Neutral-neutral word pairs were added as filler-material. The affective content of the words was rated on an electronic visual analogue scale (VAS) by eight staff members (3 men). The filler-words and the positive words were rated on a bipolar scale (from 'very negative' to 'very positive'). The depression-related words and the neutral words with which they were paired, were rated on depression relevance (from 'not at all' to 'very'). For each rater, VAS scores were normalized (Z-scores), and means and standard deviations were calculated. A final set of 36 depression-neutral pairs, 36 positive-neutral pairs, and 72 neutral-neutral pairs was selected. For the practice-session 42 extra neutral-neutral word-pairs were selected. The rating difference between words of a pair was at least 0.5 for depression-neutral pairs (mean 1.07) and positive-neutral pairs (mean 1.20), and between -0.5 and 0.5 for neutral-neutral pairs (mean 0.03 ).

Similar to a standard spatial cueing paradigm, an arrow cue was presented at fixation on every trial. Arrows were either predictive or non-predictive (neutral) of the position of the probe. Non-predictive arrows were neutral cues pointing in both directions $(<>)$ that indicated that the probe was equally likely to occur on the left or the right position. Predictive arrows were cues to the left $(<<)$ and cues to the right $(>>)$ that indicated with $79 \%$ probability the side on which the probe would occur. Therefore, in most trials predictive arrows correctly indicated the position where the probe would appear (valid trials), but in part of the trials (21\%) the opposite, incorrect, position was indicated (invalid trials). Faster reaction times (RTs) on valid trials than on invalid trials are taken as a reflection of attentional orienting and this RT difference is called "the validity effect". It is assumed that after a neutral cue attention is not oriented but kept 

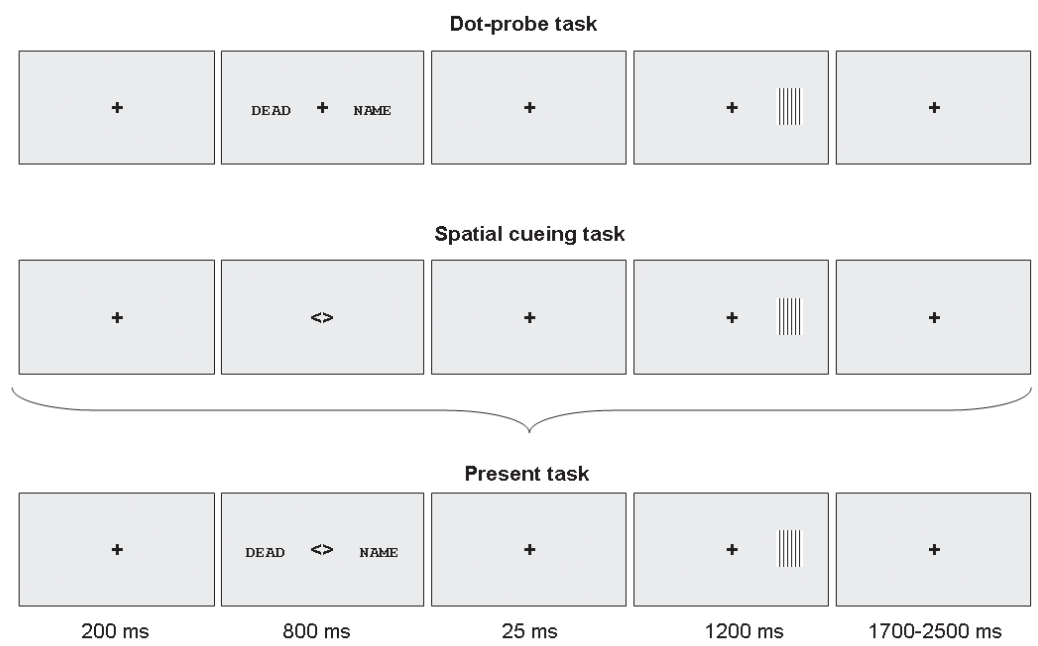

b

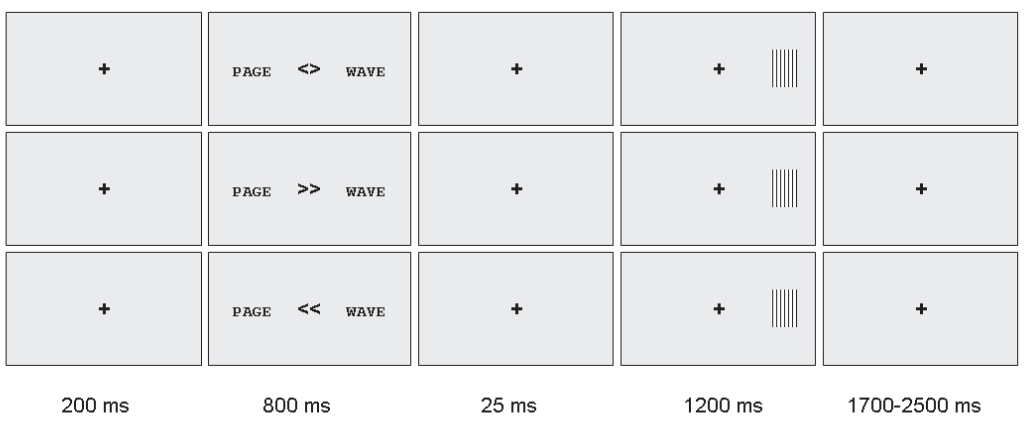

Figure 5.1 (a) The task was a combination of a dot probe task and a spatial cueing paradigm. Therefore, a word pair, with one word left and one word right, always was presented together with an arrow cue at fixation. Here, an example of a trial with an emotional-neutral word pair and a non-predictive arrow is presented. (b) Whereas emotional-neutral word pairs were always combined with non-predictive arrows, neutral-neutral word pairs were combined with non-predictive or predictive arrows: examples of trials with a neutral-neutral word pair and a neutrally cued, validly cued and invalidly cued probe to the right, respectively.

at fixation until probe-onset and RTs of neutral trials are usually in between those of valid and invalid trials. The RT-difference between neutral trials and invalid trials therefore reflects the cost of orienting attention to the wrong location and the subsequent switching of attention to the correct location. Similarly, the RT-difference of neutral trials and valid trials reflects the temporal advantage of orienting attention to the correct location. Costs and benefits have been described in terms of mental operations involving the subprocesses of orienting. Whereas benefits represent the ability to shift and engage attention in advance of probe onset, costs index the inability to disengage attention from the (invalidly) cued location. 
A word pair and an arrow cue were presented simultaneously on every trial, followed by the presentation of a probe. The probe consisted of a small black-and-white vertical square-wave-grating $\left(0.4^{\circ}, 7.9\right.$ cycles/degree) and was presented on a gray background with the same average luminance. The different types of word pairs were combined with predictive and non-predictive arrows in a way that allowed independent measurement of attentional bias for emotional words and general orienting processes within the same task. That is, emotional-neutral word-pairs, used to measure attentional bias, were only presented together with non-predictive arrow cues. Fifty percent of the trials with neutral-neutral (filler) word-pairs were presented together with predictive arrows whereas the remaining $50 \%$ of the trials was presented together with non-predictive arrows. General orienting processes were measured independent of attentional bias for emotional words by analysing all predictive arrows and only those non-predictive arrows that were combined with neutral-neutral word-pairs (see Figure 5.1b).

Participants were instructed to respond to every probe fast and accurately by pressing a button with their right index finger. They were asked to maintain central eye fixation, ignore the words, and pay attention to the arrow. They were informed about the predictive validity of the arrows and that paying attention to them would decrease their RT. The inclusion of the general orienting task thus ensured that participants would maintain central fixation and provided them with a task, other than to detect a probe and ignore word stimuli (Mathews et al., 1996). To prevent premature responses, catch trials were introduced into the design and randomly intermixed. On these trials $(22 \%$ of all trials) no probe was presented. Therefore, participants were forced to withhold their response on every trial until a probe was presented. Corrective feedback was given when participants did not respond to a probe (so called "misses"), when participants responded during a catch trial (so called "false alarms"), and when responses preceded probe-onset, were too fast $(<120 \mathrm{~ms})$ or were too slow $(>1200 \mathrm{~ms}){ }^{1}$

The experiment consisted of a 42-trial practice-block and two 144-trial experimental-blocks (plus 10 warm-up trials that were not analysed) in which all word-pairs were randomized. Participants received feedback consisting of their average RT in ms every 20 trials.

\section{Procedure}

Participants completed the BDI, the trait and state subscales of the State-Trait Anxiety Inventory (van der Ploeg, Defares, \& Spielberger, 1980), and the Hospital Anxiety Depression Scale (HADS; Spinhoven et al., 1997). They were then seated in a soundattenuated room, viewing a VGA-monitor at $75 \mathrm{~cm}$. ERTSVIPL V3.32c (Beringer, 1987) controlled the task. After the task, participants completed a Dutch shortened Profile of Mood States (POMS; Wald \& Mellenbergh, 1990).

1 For one exceptionally slow depressed patient the upper limit had to be adjusted to 1800 ms. 


\section{Results}

\section{Group characteristics}

Group characteristics are described in Table 5.1. ${ }^{2}$ Patients in the mildly depressed state scored higher on measures of anxiety and depression and lower on vigilance than both the patient group in the euthymic state and the control-group. Euthymic patients and controls did not differ on any of the depression measures but euthymic patients reported a higher level of anxiety.

\section{Errors and outliers}

False alarms (mean 5.5\%, SD 5.2\%), misses (mean 0.4\%, SD 0.97\%), and outlier-RTs (mean $2.2 \%$, SD $0.94 \%$ ), defined as RTs that deviated more than 2.5 SDs from the individual mean latency, were removed from the data. The groups did not differ in error or outlier rates. On average $96 \%$ of the data remained for analyses.

\section{Validity effects}

Table 5.2 displays mean RTs in the cueing paradigm for every group. As described in the introduction, three comparisons were of interest: (1) patients in the mildly depressed state versus the matched control group, reflecting the combination of state and trait effects; (2) patients in the euthymic state versus the matched control group, reflecting trait effects; (3) patients in the mildly depressed state versus patients in the euthymic state, reflecting state-dependent effects. For the first two comparisons a 2 (group: patient, control) $\times 3$ (validity: valid, neutral, invalid) ANOVA was carried out on RTs. The third comparison was made to examine state effects of level-of-depression within the patient group as a whole. Therefore, instead of the dichotomous between-subjects factor (mildly-depressed versus euthymic), the BDI-score was entered in the ANOVA as a continuous predictor variable. For each of the three comparisons, an effect of validity with faster RTs for validly cued than for invalidly cued trials was taken as a reflection of attentional orienting. Sub-processes of shifting and disengagement were then examined through RT benefits (i.e., faster RTs on valid relative to neutral trials) and RT costs (i.e., slower RTs on invalid relative to neutral trials), respectively. This was done for each of the first two comparisons, using a 2 (group: patient, control) $\times 2$ (validity: valid, neutral) ANOVA for benefits, and a 2 (group: patient, control) $\times 2$ (validity: invalid, neutral) ANOVA for costs. For the third comparison, instead of the dichotomous between-factor, the BDI-score again was entered in the ANOVAs as a continuous predictor variable.

Trait-plus-state effects: mildly depressed patients versus matchedcontrols. Although patients were slower (mean RT: $520 \mathrm{~ms}$ ) overall compared to controls (mean RT: $434 \mathrm{~ms}$ ), the main effect of group was not significant $(F(1,30)=2.6$, $\left.p=.12, \eta_{p}{ }^{2}=.08\right)$. As expected, there was a main effect of validity $(F(2,29)=11.1$,

2 Note that the average depression-score in the group described as "in a mildly depressed state" was not very high. Still, the group scored higher on the BDI than both the matched control-group and the patient-group in a euthymic state, therefore making it possible to draw conclusions on differences between patients in a mildly depressed state and controls and to examine differences related to level of depressed mood within the patient-group as a whole. 
Table 1 Group characteristics (SEs in parentheses) and independent t-tests

\begin{tabular}{|c|c|c|c|c|c|c|c|c|}
\hline & \multirow[b]{2}{*}{$\begin{array}{l}\text { Mildly } \\
\text { Depressed } \\
(\mathrm{n}=16)\end{array}$} & \multirow[b]{2}{*}{$\begin{array}{l}\text { Euthymic } \\
(n=13)\end{array}$} & \multirow[b]{2}{*}{$\begin{array}{l}\text { Controls-d } \\
(\mathrm{n}=16)\end{array}$} & \multirow[b]{2}{*}{$\begin{array}{l}\text { Controls-e } \\
(n=13)\end{array}$} & \multicolumn{4}{|c|}{ t-tests } \\
\hline & & & & & State & Trait & $\begin{array}{r}\text { Mildly } \\
\text { Dep } \\
\text { vs } \\
\text { con-d }\end{array}$ & $\begin{array}{r}\text { Con-d } \\
\text { vs } \\
\text { con-e }\end{array}$ \\
\hline Age & $48(6)$ & $43(8)$ & $50(7)$ & 44 (11) & ns & ns & ns & ns \\
\hline$I Q$ & $\begin{array}{l}110.6 \\
(10.3)\end{array}$ & $\begin{array}{l}118.6 \\
(12.4)\end{array}$ & $\begin{array}{l}114.1 \\
(13.6)\end{array}$ & $\begin{array}{l}113.6 \\
(12.8)\end{array}$ & ns & ns & ns & ns \\
\hline BDI & $\begin{array}{l}17.1 \\
(5.9)\end{array}$ & $\begin{array}{l}3.8 \\
(2.6)\end{array}$ & $\begin{array}{l}2.5 \\
(2.3)\end{array}$ & $\begin{array}{l}2.6 \\
(2.1)\end{array}$ & $* * *$ & ns & $* * *$ & ns \\
\hline STAI trait & $\begin{array}{l}52.2 \\
(7.6)\end{array}$ & $\begin{array}{l}38.7 \\
(8.9)\end{array}$ & $\begin{array}{l}26.5 \\
(5.0)\end{array}$ & $\begin{array}{l}28.2 \\
(6.4)\end{array}$ & $* * *$ & $* *$ & $* * *$ & ns \\
\hline STAI state & $\begin{array}{l}44.5 \\
(12.2)\end{array}$ & $\begin{array}{l}31.8 \\
(6.7)\end{array}$ & $\begin{array}{l}27.5 \\
(5.4)\end{array}$ & $\begin{array}{l}27.1 \\
(4.8)\end{array}$ & $* *$ & $*$ & $* * *$ & ns \\
\hline POMSdep & $\begin{array}{l}4.9 \\
(4.9)\end{array}$ & $\begin{array}{l}.85 \\
(1.4)\end{array}$ & $\begin{array}{l}.25 \\
(.58)\end{array}$ & $\begin{array}{l}.46 \\
(.97)\end{array}$ & $* *$ & ns & $* *$ & ns \\
\hline POMSvig & $\begin{array}{l}7.8 \\
(5.2)\end{array}$ & $\begin{array}{l}14.2 \\
(3.9)\end{array}$ & $\begin{array}{l}16.7 \\
(4.3)\end{array}$ & $\begin{array}{l}15.6 \\
(3.3)\end{array}$ & $* *$ & ns & $* * *$ & ns \\
\hline POMSten & $\begin{array}{l}8 \\
(3.8)\end{array}$ & $\begin{array}{l}4.8 \\
(2.2)\end{array}$ & $\begin{array}{l}2.2 \\
(1.7)\end{array}$ & $\begin{array}{l}2 \\
(1.2)\end{array}$ & * & $* *$ & $* * *$ & ns \\
\hline HADS a & $10.2(3.0)$ & 7.2 (1.8) & $5.4(1.8)$ & $5.5(1.9)$ & $* *$ & $*$ & $* * *$ & ns \\
\hline HADS d & $10.2(3.7)$ & $3.1(2.3)$ & $1.2(1.4)$ & $1.7(2.1)$ & $* * *$ & ns & $* * *$ & ns \\
\hline HDRS & $8.1(6.2)$ & $2(1.5)$ & - & - & $* *$ & - & - & - \\
\hline YMRS & $1.2(2)$ & $1.5(2.6)$ & - & - & ns & - & - & - \\
\hline
\end{tabular}

Note. Controls-d $=$ controls matched to mildly depressed patients; Controls-e $=$ controls matched to euthymic patients; State $=$ comparison of patient groups; Trait = comparison of the euthymic patient group and controls-e; Con-d vs con-e = comparison of control groups; BDI = Beck Depression Inventory; STAI = State-Trait Anxiety Inventory; POMS = (shortened) Profile of Mood States questionnaire: depression, vigilance and tension subscales were measured; HADS $=$ Hospital Anxiety Depression Scale: anxiety (a) and depression (d); HDRS = Hamilton Rating Scale(only in patient groups); YMRS = Young Mania Rating Scale(only in patient groups); ns = nonsignificant; ${ }^{*}<.05 ;^{* *}<.01 ;^{* * *}<.0005$. 
$p<.0005, \eta_{p}^{2}=.43$ ), with faster responses for valid trials compared to invalid trials, and neutral trials in between. There were costs $\left(F(1,30)=14.5, p=.001, \eta_{p}{ }^{2}=.33\right.$ ) and benefits $\left(F(1,30)=11.0, p=.002, \eta_{p}{ }^{2}=.27\right)$. There was no interaction between group and validity $\left(F(2,29)<1, p=.74, \eta_{p}{ }^{2}=.02\right)$, group and costs $(F(1,30)<1, p=$ $\left..56, \eta_{p}{ }^{2}=.01\right)$ or group and benefits $\left(F(1,30)<1, p=.65, \eta_{p}{ }^{2}=.01\right)$, indicating these effects were similar across groups.

Trait effects: euthymic patients versus matched-controls. Patients were slower (mean RT: $455 \mathrm{~ms}$ ) overall compared to controls (mean RT: $400 \mathrm{~ms}$ ), as reflected by a main effect of group $\left(F(1,24)=5.8, p=.02, \eta_{p}{ }^{2}=.19\right)$. Again there was an expected main effect of validity $\left(F(2,23)=4.5, p=.02, \eta_{p}^{2}=.28\right)$. There were significant benefits $\left(F(1,24)=7.9, p=.01, \eta_{p}{ }^{2}=.25\right)$ but no significant costs $(F(1,24)<1, p=$ $\left..58, \eta_{p}^{2}=.01\right)$. Again, there was no interaction between group and validity $(F(2,23)<$ $\left.1, p=.73, \eta_{p}{ }^{2}=.03\right)$, group and costs $\left(F(1,24)<1, p=.70, \eta_{p}{ }^{2}=.01\right)$, or group and benefits $\left(F(1,24)<1, p=.43, \eta_{p}^{2}=.03\right)$.

State effects: effect of level of depression in the patient-group ${ }^{3}$. Again there was an expected main effect of validity $\left(F(2,26)=9.4, p=.001, \eta_{p}^{2}=.42\right)$ and there were significant costs $\left(F(1,27)=8.3, p=.008, \eta_{p}{ }^{2}=.24\right)$ and benefits $(F(1,27)=$ $\left.8.6, p=.007, \eta_{p}^{2}=.24\right)$. There was an interaction between BDI and validity $(F(2,26)=$ $\left.4.2, p=.03, \eta_{p}^{2}=.25\right)$, BDI and costs $\left(F(1,27)=8,5, p=.007, \eta_{p}^{2}=.24\right)$, but not between BDI and benefits $\left(F(1,27)<1, p=.37, \eta_{p}^{2}=.03\right)$. Correlation analyses showed that costs increased with depression level (BDI: $r=0.49, p=.007$ ).

Table 2 Mean RTs (in ms; SE in parentheses) as a function of arrow cue validity

Group

\begin{tabular}{lllll}
\hline Validity & $\begin{array}{l}\text { Mildly- } \\
\text { Depressed }\end{array}$ & Euthymic & Controls-d & Controls-e \\
\hline Valid & $503(48)$ & $441(23)$ & $419(17)$ & $391(8)$ \\
Neutral & $514(48)$ & $462(22)$ & $432(21)$ & $403(10)$ \\
Invalid & $543(52)$ & $463(21)$ & $453(26)$ & $407(8)$ \\
Benefits & $10(4)$ & $21(10)$ & $13(6)$ & $12(6)$ \\
Costs & $29(9)$ & $1(7)$ & $21(9)$ & $4(4)$ \\
\hline \hline
\end{tabular}

Note. Controls- $d$ = the control group matched to the mildly depressed patient group; Controls$\mathrm{e}=$ the control group matched to the euthymic patient group.

3 There was a main effect of $\operatorname{BDI}\left(F(1,27)=4.6, p=.04, \eta_{p}{ }^{2}=.15\right)$, but after removal of an outlier (Cook's distance 1.8) it was not significant anymore $\left(F(1,26)<1, p=.49, \eta_{p}{ }^{2}\right.$ $=.02$ ). The effects of validity, costs and benefits as well as the reported interaction effects did not change in a meaningful way. 
In summary, all participants were able to orient their attention after onset of the arrows and showed benefits. Within the patient group, costs were positively related to level of depressed mood. Costs are inversely related to the ability to disengage attention. Therefore, as expected, the ability to disengage attention was inversely related to negative mood. Similar analyses, conducted on log-transformed error rates, showed no meaningful group differences.

\section{Attentional bias}

Attentional bias scores (e.g., Bradley et al., 1997) were calculated for each emotional wordtype and are shown for every group in Figure 5.2. Positive scores reflect a bias toward and negative scores a bias away from emotional words. The same three comparisons, explained in the introduction and for validity results, were of interest. For the first two comparisons (patients in the mildly depressed state versus the matched control group; patients in the euthymic state versus the matched control group), a 2 (group: patient, control) $\times 2$ (wordtype: positive, depression-related) ANOVA was carried out on bias scores. For the third comparison (patients in the mildly depressed state versus patients in the euthymic state), instead of the dichotomous between-subjects factor,

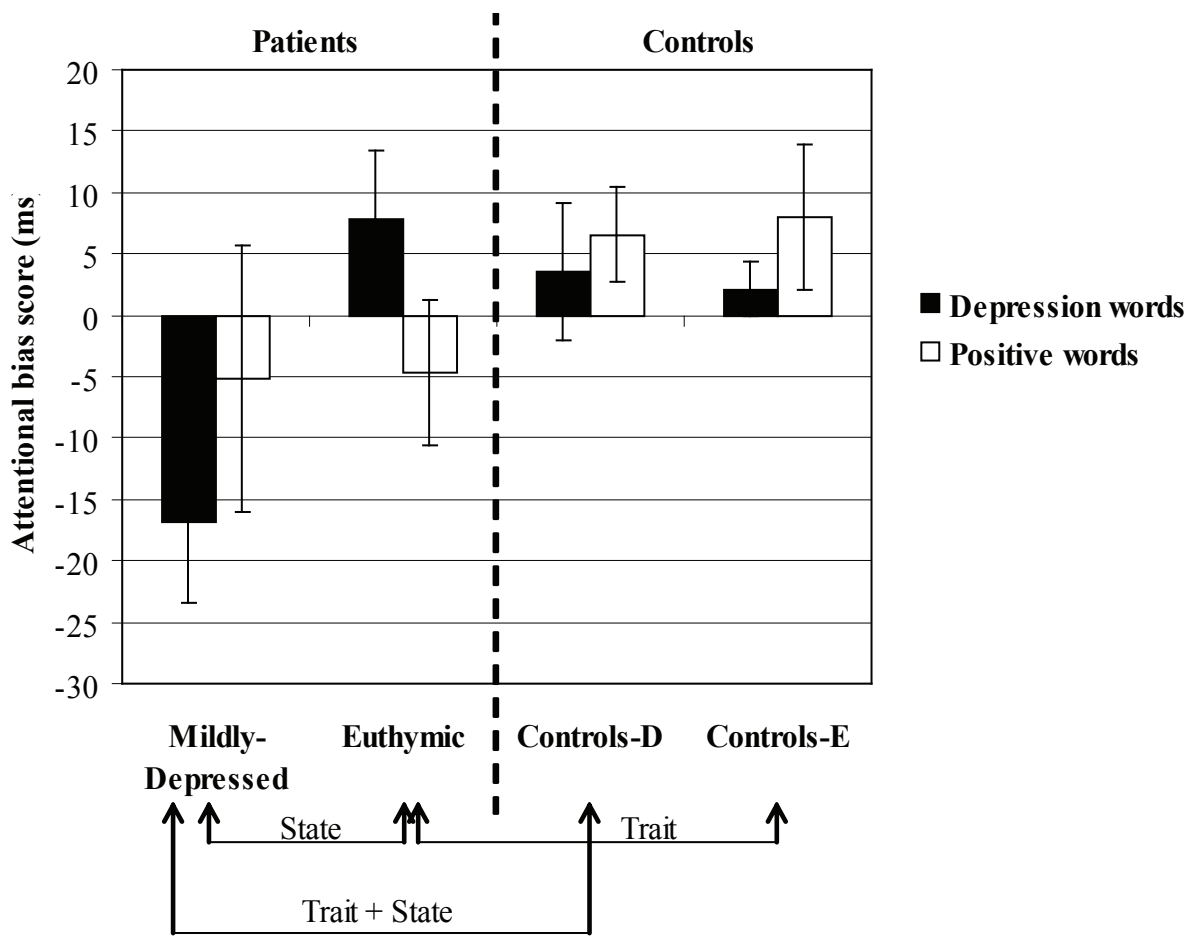

Figure 5.2 Attentional bias scores for depression-related words and positive words in the patient group that was in a mildly depressed state, the patient group in a euthymic state, and the two control groups. Error bars indicate the SE of the group average in each condition. Controls-D $=$ the control group matched to the mildly depressed patient group; Controls-E = the control group matched to the euthymic patient group. Group comparisons of interest (trait, state, trait + state) that are described in the text are indicated by arrows. 
the BDI-score was entered in the ANOVA as a continuous predictor variable. Follow-up analyses were conducted where appropriate, to test the specific hypotheses that were stated for each wordtype. For depression-related words, a bias toward these words was hypothesized for the mildly depressed group as compared to a bias away from them in the control group. For positive words a bias away from these words was hypothesized in the mildly depressed group as compared to a bias toward positive words in the control group. For each wordtype, patients in the euthymic state might either display a bias that is similar to the mildly depressed group, or they might be more similar to the healthy controls.

Trait-plus-state effects: mildly depressed patients versus matchedcontrols. There was a main effect of group $\left(F(1,30)=5.1, p=.03, \eta_{p}{ }^{2}=.14\right)$, reflecting a general bias for emotional content. That is, in comparison to the control-group, the mildly depressed patient-group showed a bias away from both types of emotional words. There was no interaction between group and wordtype $(F(1,30)<1, p=.60$, $\left.\eta_{p}^{2}=.01\right)$ and no main effect of wordtype $\left(F(1,30)=1, p=.32, \eta_{p}^{2}=.03\right)$.

Trait effects: euthymic patients versus matched-controls. There was no main effect of group $\left(F(1,24)<1, p=.54, \eta_{p}^{2}=.02\right)$ or wordtype $(F(1,24)<1, p=$ $\left..49, \eta_{p}^{2}=.02\right)$. There was a marginal interaction effect between group and wordtype $(F$ $\left.(1,24)=3.8, p=.06, \eta_{p}^{2}=.14\right)$. Whereas euthymic patients displayed a bias away from positive but toward depression-related words, controls displayed a bias toward positive words and a bias around zero for depression-related words. Follow-up independent samples t-tests for each wordtype revealed no significant group differences for positive words $(t(24)=1.5, p=.15, d=.59)$ or depression-related words $(t(24)<1, p=.35$, $d=.37)$.

State effects: effect of level of depression in the patient-group. Although Figure 5.2 suggests a large difference in bias between groups for depressionrelated words, and no difference for positive words, the interaction effect between BDI and wordtype was weak $\left(F(1,27)=2.8, p=.108, \eta_{p}{ }^{2}=.09\right) .{ }^{4}$ There was no main effect of wordtype $\left(F(1,27)<1, p=.91, \eta_{p}^{2}=.001\right)$. Given the specific hypotheses regarding depression-related words, correlation analyses between the BDI and bias scores for each wordtype were examined. There was a strong correlation between bias scores for depression-related words and BDI scores $(r=-0.63, p<.0005)$. The direction of the effect differed from what would be expected from the mood-congruency hypothesis as level of depression was related to a bias away from depression words. For positive words, there was no relation between bias scores and BDI $(r=-0.12, p=.52)$.

Taken together, attentional bias results showed a non-specific bias away from both types of emotional words in mildly depressed patients as compared with the controlgroup. The bias away from positive words was not state-dependent but acted as a trait effect that was present equally in both patient groups but not in controls. The bias away from depression-related words was state-dependent as shown by a strong negative cor-

4 There was a main effect of $\mathrm{BDI}\left(F(1,27)=6.9, \mathrm{p}=.01, \eta_{p}{ }^{2}=.20\right)$, but after removal of an outlier (Cook's distance 1.2) it was not significant anymore $(F(1,26)=2.3, p=.14$, $\left.\eta_{p}^{2}=.08\right)$. The interaction effect of wordtype and BDI was significant after removal, $F(1$, $26)=5.4, p=.03, \eta_{p}^{2}=.09$. Results from correlation analyses for every wordtype did not change in a meaningful way. 
relation between mood state and bias scores within the patient group as a whole. Similar analyses conducted on log-transformed error rates, showed no meaningful group differences regarding the bias effects reported here.

\section{Discussion}

For the first time, attentional bias in bipolar disorder was studied with a modified version of the dot-probe task. General orienting processes were measured at the same time with a spatial cueing paradigm incorporated in it.

\section{General orienting processes}

Results from the spatial cueing paradigm showed that all groups were able to orient their attention in response to arrow cues, as demonstrated by validity effects (i.e., faster RTs on valid relative to invalid trials) and benefits (i.e., faster RTs on valid relative to neutral trials). Within the patient-group as a whole, costs of attention (i.e., slower RTs on invalid relative to neutral trials), reflecting the relative inability to disengage attention, were positively related to the level of depressed mood. A similar relation between costs on a spatial cueing paradigm and negative affect in response to a distressing film was demonstrated in a nonclinical sample (Compton, 2000). One explanation that was suggested for these data was that participants with an inability to disengage attention in the orienting task also had difficulty to disengage their attention from the distressing content of the film. Problems with disengagement from negative emotional information have also been suggested to influence clinical depression, through their effect on memory or ruminative responses (Joormann, 2004; Mogg \& Bradley, 2005). Furthermore, Mathews and Mackintosh (1998) proposed a relation between general attentional control and attentional bias, suggesting that bias will be strongest in those unable to control their attention. In accordance, a relation between problems with disengagement and attentional biases has been suggested for depression (Bradley et al., 1997) and was shown in dysphoria (Ellenbogen et al., 2002; Koster et al., 2005). In sum, the current results demonstrate a positive relation between a relative inability to disengage attention from nonemotional information and measures of negative mood state in a clinical sample of patients with bipolar disorder. It will be important for future studies to determine whether this general inability to disengage attention in bipolar disorder is related to attentional bias.

\section{Attentional bias}

On the dot probe task, the mildly depressed group demonstrated an attentional bias away from both types of emotional words in comparison with controls that showed a bias toward positive words. A strong relation within the patient-group between level of depressed mood and bias convincingly showed that the bias away from depressionrelated words in the mildly depressed group was state-dependent. The bias away from positive words was not state-dependent but was equally present in both patient groups. It was part of a trait effect, demonstrated when the group of patients in a euthymic state was compared to the control group. Euthymic patients showed a bias away from positive but toward depression-related words. Findings of a study by Malhi, Lagopoulos, Sachdev, Ivanovski, and Shnier (2005) might be related to the trait effect shown here. A neural trait deficit was shown in a group of euthymic bipolar patients, reflected 
by a general attenuation of cortical and subcortical responses to emotional stimuli on an Emotional Stroop task.

A bias away from positive words in a dysphoric group and toward them in a controlgroup has been shown before (e.g., Bradley et al., 1998; Koster et al., 2005). However, a bias away from depression-related words with increasing levels of depressed mood differs from what would be expected on the basis of the mood-congruency hypothesis (e.g., Beck, 1976) and results that have been reported in unipolar depression and dysphoria (Mathews \& MacLeod, 2005). A possible explanation for this result comes from a number of studies in anxiety research, where it was shown that highly anxious participants automatically move their attention towards negative stimuli, but strategically move it away when presentation duration is longer (e.g., Koster, Verschuere, Crombez, \& van Damme, 2005; Mogg, Bradley, Miles, \& Dixon, 2004; Rohner, 2002). In the present study, words were presented long enough to allow similar strategic processes. Furthermore, even the euthymic patient-group reported higher anxiety than controls. However, although the bias away from emotional information might be related to the level of anxiety, this does not explain the absence of a similar bias in studies of unipolar depression and dysphoria because depressed mood and anxiety usually co-occur (Mineka, Watson, \& Clark, 1998). Furthermore, recent evidence from our lab showed a bias towards depression-related words in dysphoric undergraduates on the task presented here (Jongen \& Smulders, 2005). Therefore, these data suggest that biases in patients with bipolar disorder in a mildly depressed state are different from biases in dysphoria and unipolar depression.

The most important diagnostic difference between unipolar depression and bipolar disorder is the occurrence of manic and mixed episodes in the latter. Although speculative, a bias away from all emotional information might be unique for a depressed state in bipolar disorder and might be related to the changes of mood that are typical for bipolar disorder. In a prospective study it was shown that biases at one point in time could predict diagnoses of depression 18-28 months later (Rude, Valdez, Odom, \& Ebrahimi, 2003). Similarly, the bias away from all emotional information might predict a transition from the mildly depressed state to a euthymic state. In line with this, the bias away from positive and toward depression-related emotional words in the euthymic state might predict a transition from the euthymic to one of the other mood states. In sum, these data fit with our suggestion that biased attention in bipolar disorder might be related to its characteristic transition of mood states.

Since this is the first time that a dot-probe paradigm was used in bipolar disorder and the direction of attention for emotional stimuli could be determined, replication is needed. It should be noted that Lyon, Startup, and Bentall (1999) showed a bias for depression-related words in a group of bipolar depressed, using an Emotional Stroop task. In two other Emotional Stroop studies there was no RT-interference in euthymic, depressed or manic patients with bipolar disorder (Kerr, Scott, \& Phillips, 2005; Malhi, Lagopoulos, Sachdev, Ivanovski, \& Shnier, 2005). Unlike the dot-probe task, the Stroop task does not provide an unambiguous measure of selective attention. Interference may reflect competition at the attentional stage or a later stage of response selection may be affected (MacLeod, 1991; de Ruiter \& Brosschot, 1994; Williams, Mathews, $\&$ MacLeod, 1996).Therefore, the results of these studies should not be regarded as 
inconsistent with the present results as biases on both tasks may reflect different processing stages.

\section{Limitations}

It is important to acknowledge a number of limitations of this study. First, all participating patients were taking medication at the time of testing. Future studies should attempt to include unmedicated patients because medication might, among others, have an influence on emotional bias (Harmer et al., 2003). Second, it should be noted that the patient-group reported a higher level of anxiety than the control-group. Perhaps ideally, anxiety levels in the patient-group and control-group should be similar, but cases of pure depression are rare because depression and anxiety usually co-occur (Mineka, Watson, \& Clark, 1998). Due to this comorbidity and the strong relation between depression and anxiety levels, analysis of covariance is inappropriate because controlling for anxiety will also partial out true variance due to depression (Miller \& Chapman, 2001). Still, the differences in anxiety level should be kept in mind when interpreting results. Finally, no group in a manic state was included and only a mildly depressed group was tested. To further test the idea of a relation between biased attention and the transition of mood states, a longitudinal study, measuring the same patients in different mood-episodes, is needed. Inclusion of a patient-group diagnosed with unipolar depression is also necessary for direct comparison of these patients with bipolar depressed patients.

\section{Conclusions}

In conclusion, patients with bipolar disorder in a mildly depressed state showed an attentional bias away from depression-related and positive words, as compared to a healthy control group. The bias away from depression-related words was directly related to the actual depressed state. The direction of attention, away from depression-related words, differs from what has been reported for unipolar depression and dysphoria. The bias away from positive words was not state-dependent but acted as a trait effect that was present equally in both patient groups and might reflect a vulnerability factor. The biases of euthymic and mildly depressed patients together suggest a relation with the alternation of mood-states characteristic for bipolar disorder. Finally, results from the spatial cueing paradigm demonstrated that level of depressed mood in bipolar disorder was related to impaired disengagement from an attended location, even if there was no emotional content present in the field. 


\title{
The time course of general 6 orienting and attentional bias for emotional stimuli in induced and naturally- occurring dysphoria:
}

\author{
Response Times, Eye Movements, \\ and EEG
}

\begin{abstract}
Attentional biases for emotional word stimuli and general processes of attentional orienting were examined in induced and naturally-occurring dysphoria. A modified version of the dotprobe task was used and a spatial cueing paradigm was incorporated in it. To examine the time course of attention, measurement of eye movements (EMs) and EEG after cue (i.e. arrows and words) onset was combined with measurement of RTs after probe onset. Lateralized event-related potentials (ERPs) and lateralized changes in the lower alpha- 1 (Lal) band were extracted from the EEG. General orienting results showed that all participants were able to orient their attention but induced dysphoria was related to a relative inability to disengage attention. ERP results and lateralized changes in the Lal-band partly replicated previous results. Importantly, results showed that attentional bias varies over time, both within trials and between blocks of trials. We argue that this variance may have contributed to the heterogeneous results in previous studies of attentional bias in dysphoria and depression. For naturally-occurring dysphoria a bias away from depression-relevant words after word onset was followed in time by a bias toward these words or no bias, depending on task block. There was a similar bias away from positive words after word onset, but no bias in RT results. For induced dysphoria, results strongly indicated that lowered mood was related to a bias toward positive words and away from depression-relevant words. This bias may function as a means to regulate emotion.
\end{abstract}

Jongen, E.M.M. \& Smulders, F.T.Y. (in prep.) The time course of general orienting and attentional bias for emotional stimuli in induced and naturally-occurring dysphoria: response times and eye movements. 
According to cognitive models (e.g., Beck, 1976; Bower, 1981) mood-congruent biases play a causal and maintaining role in depression and dysphoria. Biases are expected to influence all aspects of information-processing. However, empirical evidence regarding attentional biases in depression and dysphoria is heterogeneous. Therefore, for some time it was thought that attentional bias was characteristic of anxiety disorders but not for depression and dysphoria (e.g. Dalgleish \& Watts, 1990; Mathews, 1990; Mathews \& Macleod, 1994; Williams, Watts, Macleod, \& Mathews, 1988). With an increasing number of studies showing evidence for attentional biases in depression and dysphoria (for reviews, see Mathews \& Macleod, 2005; Mogg \& Bradley, 2005) the role of these biases is no longer questioned. At the same time, it is not clear exactly what causes the discrepant results. First of all, it has been suggested that instead of a mood-congruent bias, depressed individuals may lack a bias for positive stimuli (Gotlib, McLachlan, \& Katz, 1988). In many studies however, positive stimuli were not included as stimulus material when attentional bias was measured. In a number of studies there was evidence for a positive bias in healthy subjects (Deldin, Keller, Gergen, \& Miller, 2001; Koster, de Raedt, Goeleven, Franck, \& Crombez, 2005; Mogg et al., 1991) and a bias away from positive stimuli in dysphoric individuals (Bradley, Mogg, Falla, \& Hamilton, 1998; Bradley, Mogg, \& Millar, 2000). Second, based on a literature review, Mogg and Bradley (2005) concluded that an important condition to obtain an attentional bias in dysphoria and depression is the opportunity for individuals to process task stimuli in an elaborate way. Biased attention in depression or dysphoria has never been demonstrated when short, masked stimulus exposure conditions were used (e.g. Bradley, Mogg, Millar, \& White, 1995; Mathews, Ridgeways, \& Williamson, 1996; Mogg, Bradley, $\&$ Williams, 1995). This has lead to the suggestion that depressed and dysphoric individuals do not automatically orient toward negative stimuli, but instead have greater difficulty, when attention has been captured, to disengage from it (Bradley et al., 1997). Finally, severity of depression and relevance of stimulus material to subjects' emotional concerns may be important conditions to obtain an attentional bias in depression and dysphoria (Mathews, Ridgeway, \& Williamson, 1996; Mogg \& Bradley, 2005). Although these factors may all be important, the discrepancy of results may also be related to the time course of attentional bias and the dependent measures that have been used to study it. As noted by others (e.g. Mogg, Millar, \& Bradley, 2000), reaction times only provide a snapshot of attention. In a dot-probe task, this snapshot is taken when the probe is presented, and attention is derived in an indirect way: through the (supposedly) faster processing of probes that are presented at attended vs. unattended locations. Since probes occur after the emotional stimuli, probe-RTs do not necessarily reflect the first shift of attention toward or away from emotional stimuli. In order to tap these first shifts of attention, a more continuous measurement of attention should ideally parallel the presentation of the word stimuli. In the present study, this was attempted for attentional bias in induced and naturally-occurring dysphoria. EMs and EEG were registered to measure the time course of attentional bias.

In addition to mood-related attentional biases, the capacity to control attention in general may also play a role in negative affect (Derryberry \& Rothbart, 1988). The process of orienting involves sub-processes of attentional shifting, engagement and disengagement (Posner, Walker, Friedrich, \& Rafal, 1984). In a nonclinical sample, results from questionnaire-data showed an inverse relation between the ability to shift 
attention and negative affect (Derryberry \& Rothbart, 1988). Similarly, those participants from a nonclinical sample who had difficulty to disengage their attention on an attentional orienting task showed the largest increase in negative affect in response to a distressing film (Compton, 2000). Finally, in a clinical sample of patients with bipolar disorder, depressed mood was associated with a relative inability to disengage attention on an attentional orienting task (Jongen, Smulders, Ranson, Arts, \& Krabbendam, in press). A similar relation between (sub-processes of) orienting and measures of negative mood may be present in induced and naturally-occurring dysphoria.

The aims of the present study were threefold. First, processes of attentional bias for emotional stimuli were investigated in induced and naturally-occurring dysphoria using a dot-probe task. Typically, in a dot-probe task two words (or pictures) of different emotional valence (e.g. one neutral and one depression-related) are presented together, side by side. Immediately following the word-pair, a probe appears at one of the positions previously occupied by a word. Participants signal the presentation of the probe by pressing a key, and their locus of attention is indicated by relatively fast responses to probes that appear at an attended location. Faster responses to probes at the previous location of a depression-related word thus would indicate an attentional bias toward depression-related words. Partly, the study was a replication of the study by Bradley et al. (1997). They measured attentional bias for depression-relevant words and anxiety-related words in a group of participants that were selected for a low score on depression measures. Half of the participants underwent a depressed mood induction procedure (MIP), and the other half a neutral MIP. In addition a group was tested that scored high on the BDI. In the present study, some changes were made. Most importantly: (1) Positive words were included because as noted before dysphorics may lack a positive bias. Furthermore, the inclusion of positive words permitted a test of the valence hypothesis versus the emotionality hypothesis. Whereas the valence hypothesis predicts biases for material only when it has negative valence (cf. Mogg et al., 1995), the emotionality hypothesis predicts biases for all types of emotional stimuli (cf. Martin, Williams, \& Clark, 1991; Mansell, Clark, Ehlers, \& Chen, 1999); (2) Whereas Bradley et al. studied naturally-occurring dysphoria and induced dysphoria in separate experiments, here a factorial design was used. In one experiment, participants that scored low or high on the BDI were subjected to a neutral or depressed MIP. This was done because according to the mood-state dependent hypothesis, cognitive vulnerability factors (i.e. attentional bias for negative stimuli / away from positive stimuli) are thought to become accessible only when they are activated by negative mood (e.g., Miranda \& Persons, 1988; Persons \& Miranda, 1992); (3) Instead of three different SOAs, only one SOA was used in the present study. As explained below, the time course of attention was studied in another way in the present study. The second aim of the present experiment was to examine general orienting processes in induced and naturally-occurring dysphoria. For this purpose, a spatial cueing paradigm (e.g., Posner, Nissen, \& Ogden, 1978), a classic measure of attentional orienting ability, was used and incorporated in the dot-probe task (hereafter referred to as spatial cueing / dot-probe task). It should be noted that the dot-probe task was derived from a spatial cueing paradigm. Typically, in one version of a spatial cueing paradigm (i.e. an endogenous spatial cueing paradigm), predictive arrow cues are presented at fixation on every trial. These arrows point to the left or to the right and are followed by a probe that appears at one of these positions. In 
most cases (e.g. 80\% of the trials), the probe appears at the position that was indicated by the arrow, but in some cases the probe is presented at the other, invalidly cued, position. Participants signal the presentation of the probe by pressing a key. Their locus of attention is indicated by relatively fast responses to probes that appear at a validly cued, attended location. The third aim of the study was to examine the time course of attention parallel to the presentation of word stimuli in the spatial cueing / dot-probe task. Therefore, in addition to response times, EMs and EEG were registered.

When measuring EMs to study attention it is important to note there is a distinction between overt and covert selection (Posner, 1980; Klein, Kingstone, \& Pontefract, 1992). Although in real life, we seem to orient our attention, by directing our eyes (overtly) toward a location of interest, it is also possible to assign priority to a location by moving attention without moving the eyes (covertly, e.g., Posner, Snyder, \& Davidson, 1980). For covert attention, to find out if attention was shifted, improvements in the efficiency of processing at attended vs. unattended locations are usually examined, for example by studying latency measures, accuracy measures, or differences in brain activity. However, covert selection and selection by EMs are closely linked. Their relation appears to be asymmetric as it is possible to shift attention without moving the eyes, but it does not appear possible to carry out an eye movement without prior shifting covert attention to the goal position of EMs (for a review, see Kowler, in press). Thus, attention is thought to be the mechanism that guides the movement of the eyes (e.g., Deubel \& Schneider, 1996; Kowler, Anderson, Dosher, \& Blaser, 1995; McPeek, Maljkovic, \& Nakayama, 1999; but see Stelmach, Campsall, \& Herdman, 1997; Rizzolatti, Riggio, Dascola, \& Umilta, 1987).

In a small number of studies, EMs have been used to examine attentional bias for emotional stimuli in depression and dysphoria. This has been done both during (passive-) viewing tasks (Eizenman et al., 2003; Matthews \& Antes, 1992) and in a dot-probe paradigm (Mogg, Millar, \& Bradley, 2000). When instructed to passively view a number of slides that each consisted of a social theme, a threatening theme, a dysphoric theme, and a neutral theme, depressed subjects elaborated longer, but not more frequently, on the dysphoric themes relative to control subjects (Eizenman et al., 2003). However, in another study, when presented with pictures consisting of happy and sad regions, both dysphorics and control subjects fixated happy regions more often, sooner, and longer than sad regions (Matthews \& Antes, 1992). In addition, when groups were compared to each other for every region, it was shown that dysphorics fixated sad regions more often than control subjects. In a dot-probe position task, there was no evidence that depressed subjects fixated sooner or more often on sad faces or away from happy faces than controls (Mogg, Millar, \& Bradley, 2000). In sum, studies that registered eye movement to study attentional bias in depression and dysphoria are scarce and the findings from these studies are heterogeneous. More studies are necessary to decide on the role of overt selection. In the present study EMs were studied as an index of attentional bias in a probe detection task, in induced and naturally-occurring dysphoria, in response to depression-relevant and positive word stimuli.

To shed light on the time course of cognitive processes, EEG has proven to be a perfect measure as it provides very precise information on the timing of neural events. In the present study, event-related potentials (ERPs) and changes in oscillations were derived from the EEG. These measures are perfectly complementary in that ERPs reflect 
only phase-locked activity and induced changes in oscillations reflect only non-phaselocked activity (Kalcher \& Pfurtscheller, 1995).

In a large number of spatial cueing studies event-related brain potentials (ERPs) have been measured to study the time course of attention and isolate components related to (effects of) attentional orienting. At first modulatory effects of attention on the processing of the target stimuli were examined (for reviews, see Anllo-Vento, Schoenfeld, \& Hillyard, 2004; Luck, Woodman, \& Vogel, 2000), but an increasing number of studies have now examined orienting time-locked to cue-onset (e.g. Eimer \& Van Velzen, 2002; Harter, Miller, Price, LaLonde, \& Keyes, 1989; Hopf \& Mangun, 2000; Jongen, Smulders, \& van Breukelen, 2006; Nobre, Sebestyen, \& Miniussi, 2000). A sequence of lateralized components with a positive or negative voltage over the hemisphere contralateral to the direction of attention has repeatedly been reported. The sequence consists of an early directing attention negativity (EDAN) at posterior electrodes between about 200 and $400 \mathrm{~ms}$ post cue, an anterior directing attention negativity (ADAN) at frontal and central sites between 300 and $500 \mathrm{~ms}$ post cue, and a late directing attention positivity (LDAP) at posterior sites that starts around $500 \mathrm{~ms}$ post cue. Although these components are suggested to reflect different stages in the control of attention, their exact functional meaning has not yet been determined. The EDAN has been hypothesized to reflect encoding of directional information provided by a cue, the resulting initiation of an attentional shift to the cued location, or both (Harter et al., 1989; Hopf \& Mangun, 2000, but see Van Velzen \& Eimer, 2003). Originally, the LDAP has been assumed to reflect modulation of the cortical excitability in those regions that process the upcoming visual information (Harter et al., 1989). However, inconsistent with this interpretation, the LDAP decreased before target onset (Hopf \& Mangun, 2000), was shown to be timelocked to cue onset instead of target onset (Van Velzen, Forster, \& Eimer, 2002), and was absent in a number of cueing studies (e.g., Mangun, 1994; Nobre et al., 2000; Talsma et al., 2005; Yamaguchi, Tsuchiya, \& Kobayashi, 1994). In line with the common view that frontal cortical structures contribute to the attentional control of sensory processing (LaBerge, 1997, Posner et al., 1987; Posner \& Petersen, 1990), the ADAN has been associated with attentional control and redirecting attention in space.

Although the exact functional meaning has not been determined yet, these components can still serve to study orienting in the present spatial cueing / dot-probe task. A comparison of conditions with arrow stimuli and conditions with word stimuli in that case might be informative. It should be noted however, that in all the studies that were mentioned, attention was directed top-down, by directional cues. Therefore, these components are hypothesized to be elicited for the spatial cueing paradigm in the present task. However, the way attention is directed by word stimuli in the dot-probe task probably differs. That is, when attention is directed by arrows cues, cue meaning is predefined and clear-cut for all participants. Obviously, this is not the case for word stimuli in a dot-probe task that are even instructed to be irrelevant to task performance. Whereas attention in a spatial cueing paradigm is directed or "pushed" to a location, attention in a dot-probe task may instead be drawn automatically to mood congruent word stimuli or alternatively may land on a word in a later processing stage. As mentioned before, there is no evidence for a preconscious bias in depression and dysphoria and it is therefore improbable that attention is drawn to the words automatically. Instead attentional bias in depression is thought to be related to later processes of selec- 
tion and disengagement. Because of these differences in the way attention is directed by word stimuli and arrow stimuli, different ERP components might also be elicited. It has been shown that selection of lateralized stimuli elicits a negative component over contralateral posterior sites, around $200 \mathrm{~ms}$ after onset of these stimuli. This so-called N2pc component was demonstrated for the first time in a visual search task, and was interpreted as a reflection of spatial filtering of irrelevant information (Luck \& Hillyard, 1994, and see also Woodman \& Luck, 1999; Woodman \& Luck, 2003). However, later studies demonstrated that it was also elicited when spatial filtering was less important, in conditions where only two stimuli were presented in opposite hemifields (Eimer, 1996; Robitaille \& Jolicoeur, 2006; Wijers, Lange, Mulder, \& Mulder, 1997). Therefore, it was suggested that the N2pc might be a reflection of detection and selection processes that are independent of the number of distracting items (Eimer, 1996). Whereas selection was based on physical characteristics in a number of these studies (Eimer, 1996, experiment 1 and 2; Luck \& Hillyard, 1994; Robitaille \& Jolicoeur, 2006), the N2pc was also elicited when selection was based on word meaning (Eimer,1996, experiment 3). Furthermore, when two words in different colours were presented and selection was based on colour, word meaning had a modulating influence on the amplitude of the N2pc (Dell'Acqua, Pesciarelli, Jolicoeur, Eimer, \& Peressotti, 2006). Especially the latter two findings lead to the hypothesis that an N2pc may be elicited upon the selection of laterally presented word stimuli in the present spatial cueing / dot-probe task.

In addition to ERP components, changes in oscillations in the alpha frequency band can also serve as indices of spatial orienting. In an endogenous cueing paradigm, an increase of alpha was shown 500 ms after cue-onset, ipsilateral to attended stimuli and contralateral to stimuli that had to be ignored, that lasted until target-onset (Worden, Foxe, Wang, \& Simpson, 2000). Increases of alpha have been interpreted as active gating of distractor stimuli and uncued spatial locations. Although the alpha band is traditionally defined as ranging from about 8 to $14 \mathrm{~Hz}$, it has been proposed that it should be divided into a number of bands, each reflecting different cognitive processes (Klimesch et al., 1998). Attentional processes would be reflected mostly by La frequencies (6.510.5). Support for this comes from recent cueing studies that reported modulation only in the La band (6-10 Hz) when attention was temporally cued (Babiloni et al., 2004), and modulation only in the La- 1 band $(6-8 \mathrm{~Hz})$ when attention was cued by position (Jongen, Smulders, \& van Breukelen, 2006). In the latter study, a spatial detection task was used that was highly similar to the spatial cueing part of the spatial cueing / dot-probe task used in the present study. Therefore analyses in the present experiment focussed specifically on changes in the La1-band. Again, arrow conditions were studied first to see if results of previous studies were replicated. Then, responses to emotional word stimuli were examined.

ERPs and event-related changes in oscillations have been used in a large number of studies to examine the time course of emotional processing in healthy, anxious and depressed participants (e.g., Deldin, Keller, Gergen, \& Miller, 2001; Fink, 2005; Holmes, Vuilleumier, \& Eimer, 2003; Schupp, Ohman, Jünghofer, Weike, Stockburger, \& Hamm, 2004). However, to our knowledge there are no studies that examined the time course of attentional bias for emotional stimuli with event-related changes in oscillations, and studies that used ERP measurement for this purpose are scarce and are limited to healthy (Pourtois, Grandjean, Sander, \& Vuilleumier, 2004; Stormark, Nordby, 
\& Hughdahl, 1995), or anxious (Bar-Haim, Lamy, \& Glickman, 2005) participants. In these ERP-studies, emotional stimuli functioned as exogenous cues (Stormark et al.) or were presented at fixation (Bar-Haim et al.). A dot-probe task was used in the study by Pourtois et al. to examine attentional bias for fearful faces in healthy participants. However, comparable to RT research, ERPs served to study modulatory processes of attention on the target. In the present study, lateralized ERP components and changes in the La1-band time-locked to the onset of depression-relevant and positive word stimuli in a spatial cueing / dot-probe task were examined and compared with activity after onset of arrow cues.

In the present study, the following hypotheses were tested:

1. Regarding attentional bias,

a. Whereas high levels of (naturally-occurring or induced) dysphoria will be associated with a bias toward depression-related words, participants scoring low on dysphoria might show a 'protective' bias, directing their attention away from negative stimuli.

b. Positive words were included to control for the possibility of a general emotionality effect (cf. Martin, Williams, \& Clark, 1991). Alternatively, the valence hypothesis predicts biases for material only when it has negative valence (cf. Mogg et al., 1995). Whereas a bias away from positive words was hypothesized for participants that scored high on dysphoria, participants scoring low on dysphoria on the other hand were hypothesized to preferentially process positive stimuli.

c. An interaction was expected between levels of naturally-occurring dysphoria and the mood induction procedure (MIP: either neutral or depressed). The depressed MIP was expected to have larger effects on those individuals that scored high on dysphoria.

2. Regarding general orienting capacity, the abilities to disengage and shift attention were expected to be inversely related to negative mood.

3. Regarding measures of the time course of attention:

a. For arrow stimuli lateralized ERP components (early posterior positivity, EDAN, ADAN, LDAP) and lateralized changes in the La1-band were expected contralateral to the direction of attention. In case the ability to shift and disengage attention is inversely related to negative mood, markers of this may be present in the EEG measures.

b. For word stimuli: ERP components might be similar to those after onset of arrow stimuli or an N2pc may be elicited after word onset. In addition similar lateralized changes in the La1-band were expected contralateral to the direction of attention and eye movements were measured.

\section{Method}

\section{Participants}

The study was approved by the institutional ethics committee. All participants gave informed consent and upon request received 20 euro or course credits for participation.

Approximately 900 student volunteers completed a Dutch version of the Beck Depression Inventory (BDI; Beck et al., 1961; Kendall et al., 1987) in a screening that was done at five faculties of Maastricht University. Recruitment tended to favour high and low BDI scorers to avoid having the majority of the sample in the mid range scores. 103 
student volunteers participated in the study. All reported normal or corrected-to-normal vision. All participants were randomly allocated to either the depressed MIP-group or the neutral MIP-group. Ten participants were excluded from the analyses: two participants did not comply with the MIP instructions, two participants had a large amount of false alarms, three participants had a large proportion of outliers (8.3-13.4\%, whereas the average percentage was $<1 \%$ ), and 3 participants had noisy EEG data. Therefore, the final sample consisted of 93 participants. Group characteristics are described in Table 6.1.

Table 6.1 Characteristics of MIP-groups

\begin{tabular}{lllll}
\hline \hline & $\begin{array}{l}\text { Neutral MIP } \\
(\mathrm{n}=43)\end{array}$ & $\begin{array}{l}\text { Depressed MIP } \\
(\mathrm{n}=50)\end{array}$ & $F(1,91)$ & $p$ \\
\cline { 2 - 5 } Gender ratio (F/M) & $35 / 8$ & $43 / 7$ & & \\
Age & 21.0 & 20.5 & $<1$ & .47 \\
BDI & 7.6 & 7.4 & $<1$ & .90 \\
STAI-trait anxiety & 41.8 & 39.4 & $<1$ & .34 \\
STAl-state anxiety & 35.8 & 35.8 & $<1$ & .98 \\
& & & & \\
\hline Note. STAI, State Trait Anxiety Inventory; BDI, Beck Depression Inventory.
\end{tabular}

\section{Task description}

The task that was used in the present study was a combination of a dot-probe task and a spatial cueing paradigm. In Figure 6.1a, this is demonstrated with an example of a trial.

Similar to a standard dot-probe task, a word pair was presented on every trial, with one word left and one word right from fixation $\left(2.3^{\circ}\right)$. Emotional-neutral word pairs consisted of a depression-related or a positive word paired with a neutral word, matched for word length and word frequency (CELEX, Baaijen, Piepenbrock, \& Gulikers, 1995). Both emotional word types were largely drawn from previous research (e.g. Bradley \& Mathews, 1983; Mathews \& Macleod, 1985; Macleod, Mathews, \& Tata, 1986) and translated into Dutch. Neutral-neutral word pairs were added as filler-material. Positive words were used to test the specificity of the attentional bias effect in depressed subjects (Dalgleish \&Watts, 1990) and to find out if healthy subjects are inclined to focus their attention on positive words. The affective content of the words was rated on an electronic visual analogue scale (VAS) by eight staff members (3 male) that were all native Dutch speakers. The filler-words and the positive words were rated on a bipolar scale (from 'very negative' to 'very positive'). The depression-related words and the neutral words with which they were paired, were rated on depression relevance (from 'not at all' to 'very'). For each rater, VAS scores were normalized (Z-scores), and means and standard deviations were calculated. Word pairs were chosen that contained words with less than 9 letters. This was necessary because in the task words were presented horizontally. A final set of 36 depression-neutral pairs, 36 positive-neutral pairs, and 72 neutral-neutral pairs was selected. The rating difference between words of a pair was 

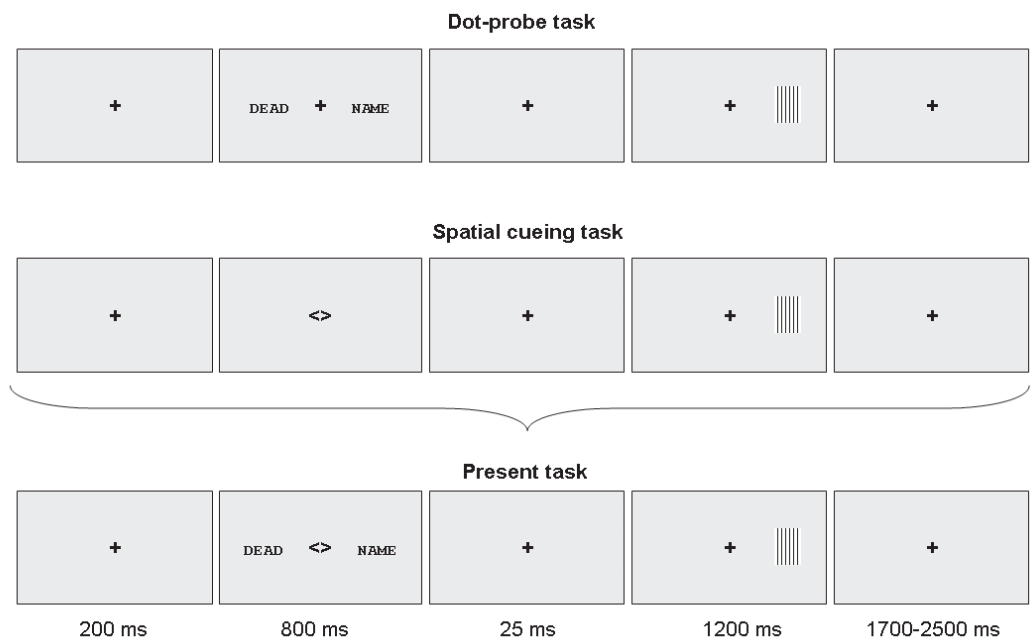

b

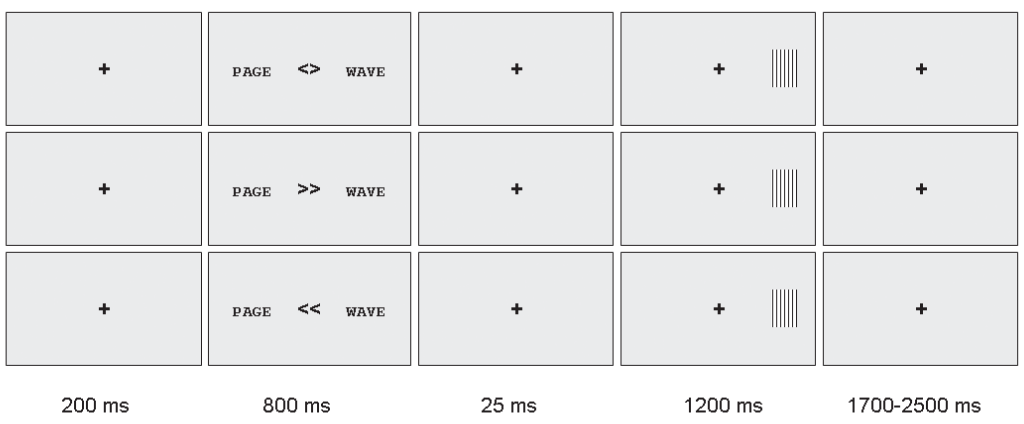

Figure 6.1 (a) The task was a combination of a dot probe task and a spatial cueing paradigm. Therefore, a word pair, with one word left and one word right, always was presented together with an arrow cue at fixation. Here, an example of a trial with an emotional-neutral word pair and a non-predictive arrow is presented. (b) Whereas emotional-neutral word pairs were always combined with non-predictive arrows, neutral-neutral word pairs were combined with non-predictive or predictive arrows: examples of trials with a neutral-neutral word pair and a neutrally cued, validly cued and invalidly cued probe to the right, respectively.

at least 0.5 for depression-neutral pairs (mean 1.07) and positive-neutral pairs (mean 1.20 ), and between -0.5 and 0.5 for neutral-neutral pairs (mean 0.03 ). For the practicesession 42 extra neutral-neutral word-pairs were selected that were only matched on word length.

Similar to a standard spatial cueing paradigm, an arrow cue was presented at fixation on every trial. Arrows were either predictive or non-predictive (neutral) of the position of the probe. Non-predictive arrows were neutral cues pointing in both directions $(<>)$ that indicated that the probe was equally likely to occur on the left or the right position. Predictive arrows were cues to the left $(<<)$ and cues to the right $(>>)$ that 
indicated with $79 \%$ probability the side on which the probe would occur. Therefore, in most trials predictive arrows correctly indicated the position where the probe would appear (valid trials), but in part of the trials (21\%) the opposite, incorrect, position was indicated (invalid trials). Faster reaction times (RTs) on valid trials than on invalid trials are taken as a reflection of attentional orienting and this RT difference is called "the validity effect". It is assumed that after a neutral cue attention is not oriented but kept at fixation until probe-onset and RTs of neutral trials are usually in between those of valid and invalid trials. The RT-difference between neutral trials and invalid trials therefore reflects the cost of orienting attention to the wrong location and the subsequent switching of attention to the correct location. Similarly, the RT-difference of neutral trials and valid trials reflects the temporal advantage of orienting attention to the correct location. Costs and benefits have been described in terms of mental operations involving the subprocesses of orienting. Whereas benefits represent the ability to shift and engage attention in advance of probe onset, costs index the inability to disengage attention from the (invalidly) cued location.

On every trial a fixation cross was presented for $200 \mathrm{~ms}$, followed by a word pair and an arrow cue that were presented simultaneously for $800 \mathrm{~ms}$. Then, after $25 \mathrm{~ms}$ of blank screen, a probe was presented. The probe consisted of a small black-and-white vertical square-wave-grating $\left(0.4^{\circ}, 7.9 \mathrm{cycles} / \mathrm{degree}\right)$ and was presented on a gray background with the same average luminance. Intertrial-interval varied between 1700 and $2500 \mathrm{~ms}$. The different types of word pairs were combined with predictive and nonpredictive arrows in a way that allowed independent measurement of attentional bias for emotional words and general orienting processes within the same task. That is, emotional-neutral word-pairs, used to measure attentional bias, were only presented together with non-predictive arrow cues. Fifty percent of the trials with neutral-neutral (filler) word-pairs were presented together with predictive arrows whereas the remaining $50 \%$ of the trials was presented together with non-predictive arrows. General orienting processes were measured independent of attentional bias for emotional words by analysing all predictive arrows and only those non-predictive arrows that were combined with neutral-neutral word-pairs (see Figure 6.1b).

Participants were instructed to respond to every probe fast and accurately by pressing a button with their right index finger. They were asked to maintain central eye fixation, ignore the words, and pay attention to the arrow. They were informed about the predictive validity of the arrows and that paying attention to them would decrease their RT. The inclusion of the general orienting task thus ensured that participants would maintain central fixation and provided them with a task, other than to detect a probe and ignore word stimuli (Mathews et al., 1996). To prevent premature responses, catch trials were introduced into the design and randomly intermixed. On these trials $(22 \%$ of all trials) no probe was presented. Therefore, participants were forced to withhold their response on every trial until a probe was presented. Corrective feedback was given when participants did not respond to a probe (so called "misses"), when participants responded during a catch trial (so called "false alarms"), and when responses preceded probe-onset, were too fast $(<120 \mathrm{~ms})$, or were too slow $(>1200 \mathrm{~ms})$.

The experiment consisted of a 42-trial practice-block and four 144-trial experimental-blocks (plus 10 warm-up trials that were not analysed) in which all word-pairs were 
randomized. Participants received feedback consisting of their average RT in ms every 20 trials.

\section{Eye movement calibration task}

An eye movement calibration task was used to determine for every subject what voltage-level corresponded with an eye movement to the left and the right probe positions in the main task. Subjects were asked to move their eyes from a central fixation mark to a probe that was presented $(1000 \mathrm{~ms})$ to the left or to the right (18 trials each). The probe stimulus was a black square with the same size as the probe from the main task. Left and right probe positions equaled the positions of the probe and words in the main task.

Mood Induction Procedure. A depressed MIP and a neutral MIP were used. In the depressed MIP-group, subjects were instructed to put themselves in a sad mood by recalling unhappy memories from their past, while listening to sad music (Prokofiev's Russia under the Mongolian Yoke, played at half speed) for 7 minutes. In the neutral MIP-group, subjects were asked to relax and listen to music (Fauré's Ballad for piano and orchestra (opus 19)) for 7 minutes. In both conditions subjects were asked to write down 3 words that reflected what they were thinking about during listening. These pieces of music have shown to be effective in manipulating mood in previous studies (e.g. Albersnagel, 1988; Clark \& Teasdale, 1985; Sutton, Teasdale, \& Broadbent, 1988). Mood measurement. A set of questionnaires were used to measure mood in the lab. These included Dutch versions of: the BDI, the state and trait versions of the State Trait Anxiety Inventory (STAI, van der Ploeg, Defares, \& Spielberger, 1980), and a shortened version of the Profile of Mood States (POMS; Wald \& Mellenbergh, 1990) consisting of six depression, six tension, and six vigour items. To monitor variations in mood over the course of the session, the shortened version of the POMS and three visual analogue scales (VASs) were used, assessing 'sad', 'happy', and 'anxious' mood state (Clark \& Teasdale, 1985). Each scale consisted of a line with two anchor points, 0 (not at all) and 100 (extremely), on which subjects could indicate how they felt "at this moment".

\section{Procedure}

A summary of the procedure is given in Figure 6.2. Upon entering the lab, subjects were asked to fill out the set of questionnaires (BDI, trait and state versions of the STAI, shortened version of the POMS). After the preparations for the EEG recordings, participants were asked to take place in a dimly lit, sound attenuated room. Tasks were presented on a VGA monitor that was placed outside the room behind a window, at a viewing distance of $75 \mathrm{~cm}$. ERTSVIPL V3.32c (Beringer, 1987) controlled the task. First, two 3-minutes long measures of EEG in resting state were done, one with eyes open and one with eyes closed. These data will be discussed elsewhere. After that, participants performed the eye movement calibration task. Then, a blink calibration task was presented in which spontaneous blinks were promoted by demanding constant fixation to detect slow color changes of a fixation cross. A blink correction factor was derived from this task and was used for offline correction of trials with eyeblinks in the attention-tasks. After these calibration tasks, the dot-probe task was presented. Before the practice session of the spatial cueing / dot-probe task, mood was measured with the first set of VAS scales. After the practice session, subjects underwent the MIP that was followed by measurement of mood with the second set of VAS scales and the second 
POMS. Subjects subsequently completed the first two blocks of the spatial cueing / dotprobe task, immediately followed by the third set of VAS scales. They were then given a mood booster procedure to maintain induced mood. The instructions were the same as before, but the duration was shortened to three minutes and nothing had to be written down. After the mood reboost, mood was measured again, with the fourth set of VAS scales. Subjects then completed the third and the fourth block of the spatial cueing / dot-probe task, followed by the fifth set of VAS scales. In the depressed MIP condition, subjects engaged in a positive MIP (listening to Coppelia by Delibes) to counteract any residual effects of the mood induction.

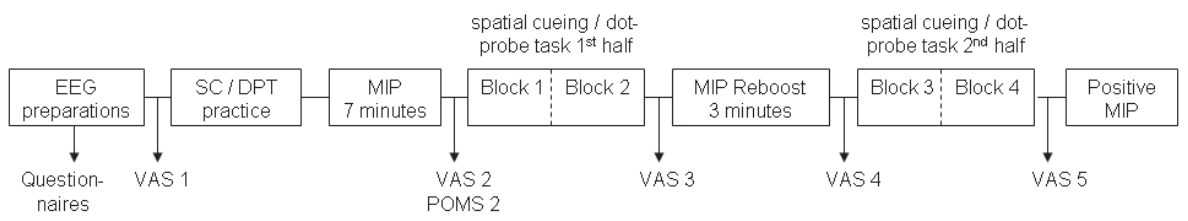

Figure 6.2 Summary of the procedure that was followed in this experiment.

\section{Data recording}

Electroencephalographic (EEG) activity was recorded continuously, via Neuroscan 4.3, from 30 scalp locations (midline: Fz, FCz, Cz, Pz, Oz; lateral: FP1/FP2, F3/F4, F7/F8, FC5/ FC6, C3/C4, CP5/CP6, T7/T8, P3/P4, P7/P8, P9/P10, P07/PO8, O1/O2; and right mastoid A2) using tin electrodes mounted on an elastic cap (Quik-Cap). A1 and A2 were used as reference and data were mathematically re-referenced offline to the average of $A 1$ and A2. AFz was used as the ground. Tin electrodes were also used to bipolarly record the vertical (above and below the left eye) and horizontal (at outer canthi of both eyes) electro-oculograms (EOGs). Electrode impedance was kept below $5 \mathrm{k} \Omega$, amplifier bandpass was $0.05-100 \mathrm{~Hz}$, and digitisation rate was $500 \mathrm{~Hz}$.

Trials with eyeblinks were corrected with a regression procedure (Semlitsch, Anderer, Schuster, \& Presslich, 1986), using the blink correction factor derived from the blink calibration task. Trials with an EEG voltage exceeding $100 \mu \mathrm{V}$ were excluded from the analyses. ${ }^{1}$ Similarly, trials that contained an error or an outlier were deleted. Possible errors were false alarms, misses, responses on the cue, and responses on the target that were either faster than $120 \mathrm{~ms}$ or slower than $1200 \mathrm{~ms}$. After examining RT distributions, trials with RTs greater than 800 ms were excluded as outliers.

1 Exclusion of trials with eye movements by following the procedure that was used in chapters 2 and 3 of this thesis was not possible in the present study because a criterion of $30 \%$ (and even 100\%) of the median eye movement, determined in the eye calibration task, did not discriminate real eye movements from noise on the HEOG channel in a reliable way. Instead, a large (and possibly random) percentage of trials were lost in that case. This was caused by the smaller distance between word cues/ the probe and fixation in the present task (2.3 degrees versus 5.4 degrees in chapter 2 and 6.8 degrees in chapter 3 ). When a criterion of $+/-70 \mu \mathrm{V}$ was applied on the HEOG channel, the percentage of trials that was lost was very small, but trials still were rejected randomly. Therefore, no criterion was used on the HEOG channel. However, to verify that ERP results are not the result of systematic eye movements, the HEOG channel will always be included for inspection. 
Whereas reaction time analyses and analyses of ERPs and EEG frequency bands were all performed on the data set that resulted after trial exclusion, analyses of eye-movements were performed on the total number of trials $(n=576)$. For every subject, voltage levels corresponding to an eye movement to the left and to the right probe / word position were derived from the eye movement calibration task. For both the main task and the eye movement calibration task, data of the HEOG channel was re-filtered with a $40 \mathrm{~Hz}$ (24 dB/oct.) low pass filter. The EEG was epoched into 1000-ms periods, starting $200 \mathrm{~ms}$ before cue onset, and the $200 \mathrm{~ms}$ pre-cue interval was used as a baseline. For the dot-probe task, individual averages for words on the left and the right position were computed for each emotional wordtype. Trials with depression-relevant - neutral word pairs and positive - neutral word pairs thus provided the data of interest. For the eye movement calibration task averages for the left and the right probe position were computed. Additional smoothing of the signal was done by applying an $8 \mathrm{~Hz}$ (24 $\mathrm{dB} /$ oct.) low pass filter on data of both tasks.

For analyses of ERPs and event-related changes in EEG frequency bands, the EEG was epoched into $1000 \mathrm{~ms}$ periods, starting $200 \mathrm{~ms}$ prior to the cue, and the $200 \mathrm{~ms}$ precue interval was used as a baseline. For ERP analyses, averages were computed for arrows to the left and arrows to the right and for positive and depression-relevant words on the left and the right position. After averaging, the data were filtered by applying an $8 \mathrm{~Hz}$ (24 dB/oct.) low pass filter. For frequency band analyses, absolute amplitude changes were computed following the "temporal spectral evolution" method (Salmelin \& Hari, 1994). Bandpass filtering (Butterworth, zero phase, $96 \mathrm{~dB} / \mathrm{oct}$ ) was done at the continuous file. After epoching, the filtered ERP was computed and subtracted to compute induced epochs. This served to discriminate phase-locked from non-phase-locked event-related EEG activity (Klimesch, Russegger, Doppelmayr, \& Pachinger, 1998). After baseline correcting induced epochs for every condition with the pre-stimulus interval, data were rectified. Averages were computed for arrows to the left and arrows to the right and for positive and depression-relevant words on the left and the right position. Finally, averages were smoothed with a 71 point moving average filter, and a baseline correction was done using the pre-cue interval.

\section{Data analysis}

MIP-effect on mood. First, MIP-groups were compared before the mood manipulation when no group differences were expected. To assess the effect of the MIP on mood state, data of each of the POMS subscales were entered in an ANOVA with between-subjects factors MIP-group (2: neutral, depressed) and BDI-score (entered as a continuous predictor variable), and within-subjects factor time (2: before MIP, after MIP). Similarly, data of each of the VAS scales were entered in an ANOVA with between-subjects factors MIP-group (2: neutral, depressed) and BDI-score (entered as a continuous predictor variable), and within-subjects factor time (5: before MIP, after MIP, before mood re-boost, after mood re-boost, immediately after the spatial cueing / dot-probe task).

Attentional bias. Attentional bias scores were calculated for each emotional word type (depression-relevant, positive) by subtracting the average RT of trials in which the emotional word and the probe appeared on corresponding positions from the average RT of trials in which the emotional word and the probe appeared on non- 
corresponding positions. As a result, positive scores reflect a bias toward the emotional word and negative scores reflect a bias away from the emotional word.

An ANOVA was carried out on these scores. The spatial cueing / dot-probe task consisted of four blocks. These blocks can be described in a 2-by-2 design, as shown in Figure 6.2. That is, two of the blocks followed immediately on the MIP and the MIP re-boost (i.e. block 1 and block 3, respectively). The other two blocks in time were further away of the MIP and MIP-reboost (i.e. block 2 and block 4, respectively). Therefore one of the factors in the design is time interval after the MIP (2: short, long), after this indicated as "MIP-interval". In addition two of the four blocks preceded the MIP reboost and were part of the first half, whereas the other two followed on the MIP reboost and were part of the second half. Therefore, the second factor in the design is task-half (2: first half, second half). In addition to task-half and MIP-interval, wordtype (2: depression-relevant, positive) was entered as a within-subjects factor, and MIP-group (2: neutral, depressed) and BDI-score (as a continuous predictor variable) were entered as between-subjects factors.

General Orienting. Results of the four blocks were pooled because the number of invalid trials in every block was too small to examine each of the four blocks separately. The RT data were entered in an ANOVA with between-subjects factors MIP-group (2: neutral, depressed) and BDI-score (entered as a continuous predictor variable), and within-subjects factors validity (3: valid, neutral, invalid) and target (left, right).

Eye-movement analyses. Electrode-placement in the present experiment was such that eye movements to the left lead to an increase in HEOG-voltage and eye movements to the right lead to a decrease in HEOG-voltage. For each emotional word type, a subtraction was made of averages of trials in which the emotional word was presented to the left and trials in which the emotional word was presented to the right (left minus right). This way, a positivity reflects an eye movement toward the emotional word, and a negativity reflects an eye movement away from the emotional word. The time-window of interest (cue-target interval: 0-800 ms) was divided into two latency windows of $400 \mathrm{~ms}$. For every individual mean voltages within these windows were corrected with data from the eye movement calibration task. This was done, because (absolute) horizontal EOG amplitude depends on head shape and exact electrode position. Left and right averages from the calibration task therefore were also subtracted from each other and peak detection was done in the interval between 0 (probe onset) and 800 ms. Mean voltages from the spatial cueing / dot-probe task were divided by the peak voltages and these proportions were analyzed in a mixed design ANOVA. In addition to task-half, MIP-interval, and wordtype, latency window (2: 0-400 ms, 400-800 ms) was entered as a within-subjects factor. MIP-group and BDI-score again were entered as between-subjects factors.

ERPs and Event Related Changes in the La1-band. The latency window between 0 and $800 \mathrm{~ms}$ relative to cue onset was analyzed. The main goal of this study was to measure attentional orienting in response to emotional word stimuli immediately after the presentation of emotional words. In addition, attentional orienting in response to arrow stimuli was examined for two purposes: first, to find out if previous findings were replicated, and second, to compare EEG measures of attentional orienting for arrows and for word stimuli. For ERP analyses, the window was divided into four successive latency windows (150-300 ms; 200-400 ms; 300-500 ms; 500-800 ms) based 
on previous findings (Harter et al., 1989; Jongen, Smulders, \& van Breukelen, 2006; Luck \& Hillyard, 1994; Mangun, 1994; Van Velzen \& Eimer, 2003). Mean voltages within these windows were used as dependent variables in three separate regional analyses. The three regions consisted of anterior (F7/8, F3/4), central (FC5/6, C3/4, CP5/6) and posterior (P3/4, P7/8, P9/10, PO7/8, 01/2) sites. Between 300 and 500 ms, a negativity was expected at frontal and central sites (ADAN) and at posterior sites (EDAN). Between 150 and $300 \mathrm{~ms}$, a positivity was expected at posterior sites (Jongen, Smulders, \& van Breukelen, 2006). Between 200 and $400 \mathrm{~ms}$, a negativity, the N2pc, was expected, but only when word stimuli were presented. Finally, at posterior sites a late positivity (500800 ms; LDAP) was expected. For analyses of event-related changes in the La1-band, successive mean voltage values every $200 \mathrm{~ms}$ were used as dependent variables in the posterior region. A lateralized decrease in the La1-band was expected at posterior sites, contralateral to the direction of attention, based on previous findings (Jongen, Smulders, \& van Breukelen, 2006). ERP mean amplitudes and La1-band mean voltage values were analyzed in a mixed design ANOVA. There were three within factors: cued direction of attention (2: left, right), hemisphere (2: left, right), and electrode (2, 3 or 5, depending on the region). MIP-group and BDI-score were entered as between-subjects factors. These analyses were done separately for arrow cues, depression-relevant words, and positive words. In these analyses, the presence of a lateralized effect sensitive to the direction of an attentional shift would be reflected by the interaction between the cued direction of attention and hemisphere. Interactions between any other factor and cue and hemisphere will be assessed separately for every level of this other factor. The Greenhouse-Geisser epsilon correction factor was applied to compensate for possible effects of non-sphericity in the measurements compared. Only the corrected F- and probability values and the uncorrected degrees of freedom are reported.

\section{Results}

\section{Group characteristics}

Group characteristics are described in Table 6.1. Importantly, there were no group differences in anxiety and dysphoria scores.

\section{Errors and outliers}

False alarms (mean 7.3\%, SD 6.5\%), misses (mean $0.1 \%$, SD $0.4 \%$ ), responses to the cue (mean $0.3 \%$, SD $0.4 \%$ ), responses faster than $120 \mathrm{~ms}$ or slower than $1200 \mathrm{~ms}$ (mean $0.1 \%$, SD $0.2 \%$ ), outlier-RTs (mean $0.5 \%$, SD $0.7 \%$ ), and trials with EEG artefacts (mean $1.5 \%$, SD $2.9 \%$ ) were removed from the data. On average, $96.1 \%$ of the data remained for analyses.

\section{MIP effect on mood}

POMS scores and VAS scores for the neutral MIP-group and the depressed MIP-group are shown in Table 6.2 and Table 6.3, respectively. Group comparisons are made for each time point. Before the mood manipulation, the depressed MIP-group and the neutral MIP-group did not differ on any of the POMS subscales. There was no interaction between MIP-group and BDI in any of the analyses. Therefore, analyses were run without the interaction component. After the MIP participants in the depressed MIP-group 
scored higher on depression and tension-anxiety and lower on vigour than participants in the neutral MIP-group (interaction MIP-group $\times$ time: depression: $F(1,90)=97.9$, $p<.0005$; tension-anxiety: $F(1,90)=8.9, p=.004$; vigour: $F(1,90)=23.6, p<$ $.005)$.

Table 6.2 MIP effect on mood measured by POMS

\begin{tabular}{|c|c|}
\hline $\begin{array}{l}\text { Neutral MIP } \\
(\mathrm{n}=43)\end{array}$ & $\begin{array}{l}\text { Depressed MIP } \\
(\mathrm{n}=50)\end{array}$ \\
\hline
\end{tabular}

POMS-depression

$\begin{array}{ccccc}\text { Time } 1 & 3.1 & 2.7 & <1 & .77 \\ \text { Time } 2 & 2.5 & 9.8 & 99.7 & <.0005\end{array}$

POMS-anxiety

Time 1

6.2

5.2

$<1$

.49

Time 2

5.6

6.7

6.0

.02

POMS-vigour

Time 1

11.9

12.7

$<1$

.49

Time 2

8.3

5.1

15.1

$<.0005$

Note. POMS, (shortened) Profile of Mood States questionnaire: depression, vigilance and tension subscales were measured; Time 1 = before MIP; Time 2 = after the MIP.

VAS scales were presented five times to assess mood change during the session. Before the mood manipulation, the depressed and the neutral MIP-groups did not differ in sadness and anxiety. However, the depressed MIP-group scored slightly higher on happiness than the neutral MIP-group. There was no interaction between MIP-group and BDI in the analyses of happiness and anxiety. Therefore, these analyses were run without the interaction component. The results showed an interaction between MIPgroup and time for each of the VAS measures (happiness: $F(4,86)=37.4, p<.0005$; sadness: $F(4,85)=34.2, p<.0005$; anxiety $F(4,86)=5.5, p=.001)$. As shown in Figure 6.3, there was an interaction of MIP-group $\times$ BDI $\times$ time $(F(4,85)=3.4, p=$ $.01)$ for sadness. Groups representing low and high scorers on the BDI were formed on the basis of a median-split (median BDI-score: 7.0) of the present sample and the analysis of MIP-group $\times$ time was repeated for each of the BDI groups. There was an interaction between MIP-group and time in each of the groups (low-scorers: $F(4,37)=$ 39.3, $p<.0005$; high-scorers: $F(4,45)=11.2, p<.0005)$, but the effect was stronger in the group of low-scorers. Inspection of the means for each group showed that in the group of high-scorers there was a slight increase in sadness after the MIP not only in the neutral MIP-group but also in the depressed MIP-group. Consequently, the difference between the MIP-groups was smaller in the group of high-scorers than in the group of 
low-scorers, causing the interaction of MIP-groups and BDI over time for the sadness scale. As shown in Table 6.3, at each of the four assessment points after the MIP the depressed MIP-group scored higher on sadness and lower on vigour and happiness than the neutral MIP-group. With regard to happiness and sadness scores, there was a significant difference at every point of assessment, except for the third point where the difference between groups was only trend-level.

In sum, the mood induction procedure was successful as participants from the depressed MIP-group reported feeling more depressed and sad after the MIP than the neutral MIP-group. In addition the group scored lower on happiness and vigour and higher on anxiety. These effects sustained over the course of the task and moreover were largely independent of BDI scores.

Table 6.3 MIP effect on mood measured by VAS scales

$\begin{array}{lll}\begin{array}{l}\text { Neutral MIP } \\ (\mathrm{n}=42)\end{array} & \begin{array}{l}\text { Depressed MIP } \\ (\mathrm{n}=50)\end{array} & F(1,89)\end{array}$

VAS-happiness

$\begin{array}{lllll}\text { Time 1 } & 55.8 & 67.7 & 9.0 & .004 \\ \text { Time 2 } & 55.3 & 30.5 & 38.6 & <.0005 \\ \text { Time 3 } & 45.4 & 39.4 & 3.5 & .065 \\ \text { Time 4 } & 52.2 & 24.2 & 68.6 & <.0005 \\ \text { Time 5 } & 43.9 & 33.7 & 7.0 & .010\end{array}$

VAS-sadness

$\begin{array}{lllll}\text { Time 1 } & 15.1 & 17.8 & 2.4 & .12 \\ \text { Time 2 } & 22.0 & 65.6 & 109.9 & <.0005 \\ \text { Time 3 } & 15.7 & 46.6 & 3.4 & .068 \\ \text { Time 4 } & 17.1 & 68.5 & 177.9 & <.0005 \\ \text { Time 5 } & 17.8 & 45.9 & 36.4 & <.0005\end{array}$

VAS-anxiety

$\begin{array}{lllll}\text { Time 1 } & 8.8 & 9.5 & <1 & .39 \\ \text { Time 2 } & 12.0 & 28.4 & 16.0 & <.0005 \\ \text { Time 3 } & 6.5 & 16.3 & 11.5 & .001 \\ \text { Time 4 } & 5.0 & 24.4 & 28.3 & <.0005 \\ \text { Time 5 } & 4.0 & 15.5 & 18.0 & <.0005\end{array}$

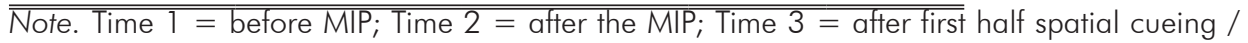
dot-probe task; Time 4 = after mood re-boost; Time $5=$ after the second half of the spatial cueing / dot-probe task. 


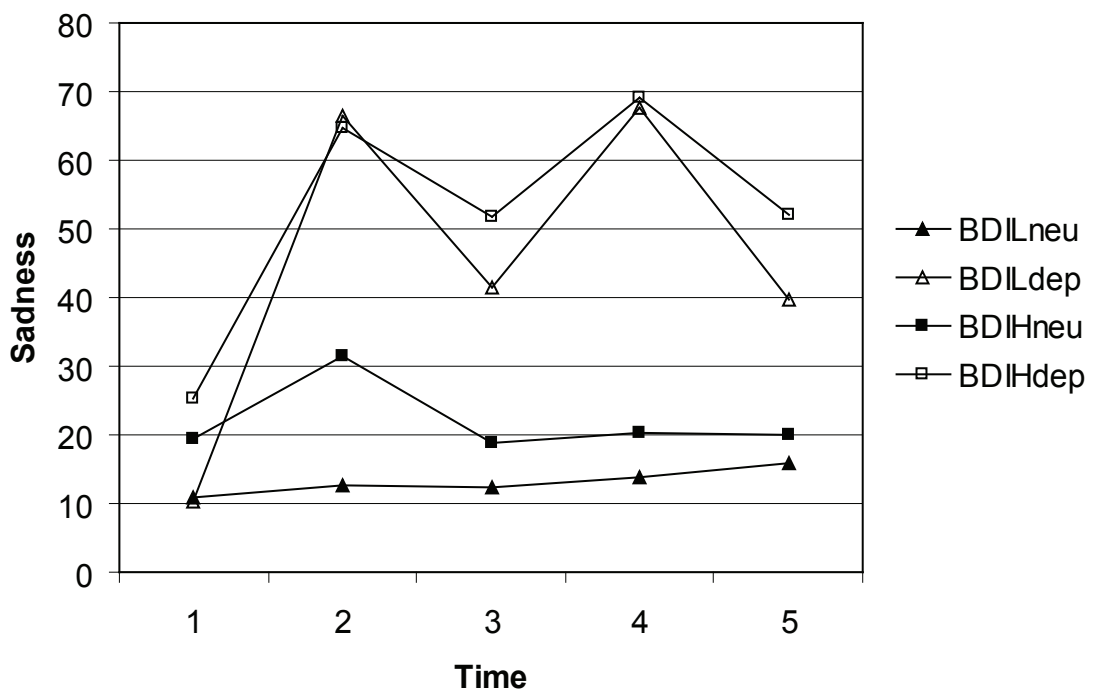

Figure 6.3 VAS measures of sadness in the neutral (neu) and the depressed (dep) MIP group. By applying a median split on the BDI, each of the MIP groups was divided into a group of low-scorers on the BDI (BDIL) and a group of high scorers on the BDI (BDIH). Figure 6.3 VAS measures of sadness in the neutral (neu) and the depressed (dep) MIP group. By applying a median split on the BDI, each of the MIP groups was divided into a group of low-scorers on the $\mathrm{BDI}(\mathrm{BDIL})$ and a group of high scorers on the BDI (BDIH).

\section{General orienting}

Table 6.4 displays mean RTs in the cueing paradigm for every MIP-group as a function of the relevant task variables. There was no interaction between MIP-group and BDI. Therefore, analyses were run without the interaction. On average, responses from the depressed MIP-group (mean $397 \mathrm{~ms}$ ) were slower than responses from the neutral MIPgroup $(377 \mathrm{~ms})(F(1,90)=3.7, p=.057)$. As expected, there was a main effect of validity $(F(2,89)=48.8, p<.0005)$, with faster responses for valid trials than invalid trials, and neutral trials in between. There were significant costs (invalid versus neutral: $F(1$, $90)=8.4, p=.005$ ) and benefits (valid versus neutral: $F(1,90)=90.4, p<.0005$ ). Interestingly, there was an interaction between MIP-group and costs $(F(1,90)=4.4$, $p=.04)$. Costs were larger in the depressed MIP-group than in the neutral MIP-group. When tested separately for each MIP-group, it was shown that costs differed from zero in the depressed MIP-group $(F(1,48)=14.8, p<.0005)$, but not in the neutral MIPgroup $(F(1,41)<1, p=.62)$.

In sum, all participants were able to orient their attention after onset of the arrows and showed benefits of attention. There were costs only in the depressed MIP-group. Costs are inversely related to the ability to disengage attention. Therefore, as expected, the ability to disengage attention was inversely related to (induced) negative mood. 
Table 6.4 Validity effects in the neutral and the depressed MIP-group

\begin{tabular}{lll}
\hline \hline & Neutral MIP-group & Depressed MIP-group \\
\cline { 2 - 3 } Valid & 362 & 378 \\
Neutral & 383 & 400 \\
Invalid & 386 & 415 \\
Benefits (neutral-valid) & 21 & 22 \\
Costs (invalid-neutral) & 3 & 15 \\
\hline \hline
\end{tabular}

\section{Attentional bias}

Whenever there was no interaction effect of MIP-group and BDI, analyses were run without the interaction effect. Results were highly dependent on wordtype, task-half, and MIP-interval. This was confirmed by statistics showing that effects of task-half $\times$ MIP-group $(F(1,90)=4.7, p=.03)$, MIP-interval $(F(1,90)=4.3, p=.04)$, and task-half $\times$ MIP-interval $\times$ BDI $(F(1,90)=5.3, p=.02)$ were superseded by an interaction of task-half $\times$ MIP-interval $\times$ MIP-group $\times$ wordtype $(F(1,90)=6.8, p=.01)$, and taskhalf $\times$ MIP-interval $\times$ BDI $\times$ wordtype $(F(1,90)=4.8, p=.03)$. To test the specific hypotheses that were stated for each wordtype, attentional bias scores for each word type were entered in an ANOVA (task-half $\times$ MIP-interval $\times$ MIP-group $\times$ BDI-score). For depression-related words, a bias toward these words was hypothesized for the (naturally-occurring and induced) dysphoric groups as compared to a bias away from them in the control group. For positive words a bias away from these words was hypothesized for the (naturally-occurring and induced) dysphoric groups as compared to a bias toward positive words in the control group. In other words, main effects of MIP-group and BDI were expected for analyses of both wordtypes. In addition, the effect of negative mood induction may be larger for those scoring high on the BDI. Therefore, there might be an interaction of MIP-group and BDI.

Depression-relevant words. For depression-relevant words, there was an interaction between task-half $\times$ MIP-interval $\times \operatorname{BDI}(F(1,90)=8.2, p=.01)$. Therefore, analyses were done separately for every task-half and MIP-interval.

Analyses for each task-half (MIP-interval $\times$ MIP-group $\times$ BDI) showed significant results only for the first task-half. An overall bias toward depression-relevant words (intercept: $F(1,90)=4.4, p=.04$ ) was superseded by an interaction of MIP-interval (i.e. block 1 versus block 2$) \times \operatorname{BDI}(F(1,90)=5.0, p=.03)$. Correlation analyses showed that in the second block, following our expectations, there was a positive correlation between BDI and bias scores $(r=0.20, p=.05$; first block: $r=-0.12, p=.26)$ as shown in Figure 6.4a.

Analyses for each MIP-interval (task-half $\times$ MIP-group $\times$ BDI) showed effects only for the long interval (block 2 and block 4). Firstly, there was an interaction of task-half (i.e. block 2 versus block 4$) \times \operatorname{BDI}(F(1,90)=4.8, p=.03)$. Analyses on each block (MIP-group $\times$ BDI-score) showed an effect of BDI in the second block $(F(1,90)=3.9$, $p=.05)$, indicating the positive correlation between BDI and bias scores for depression-relevant words that was mentioned already. There was no effect of BDI in the fourth block $(F(1,90)=1.9, p=.18)$. Secondly, there was an interaction of task-half 
a

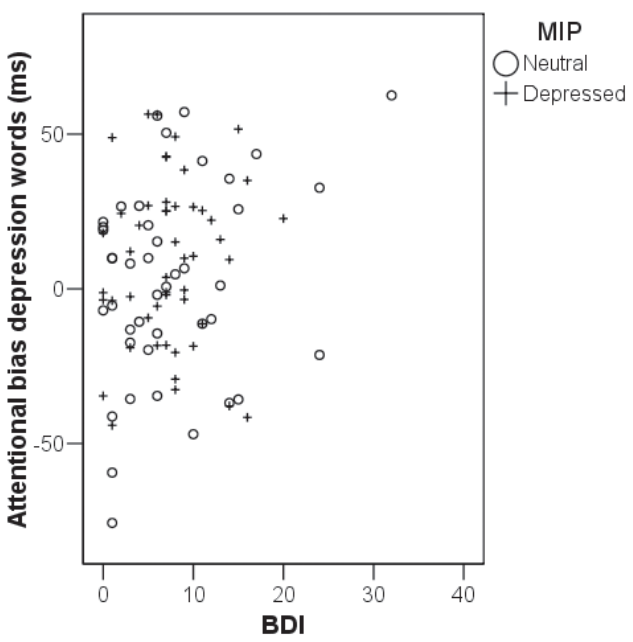

b

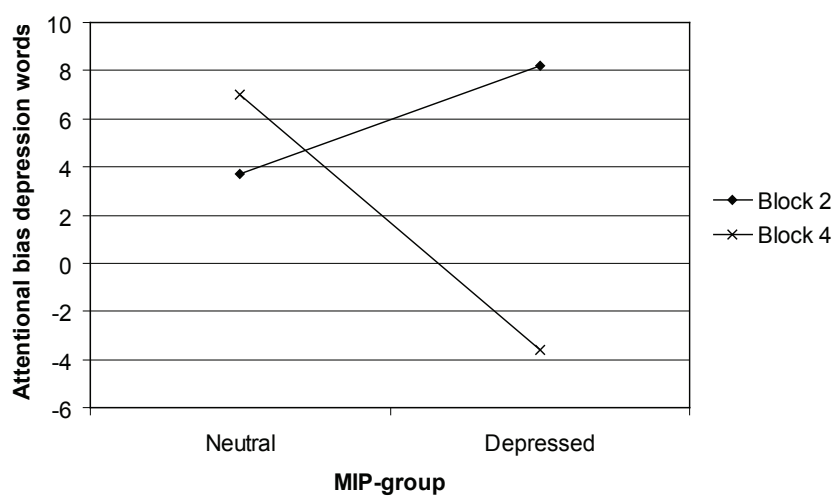

C

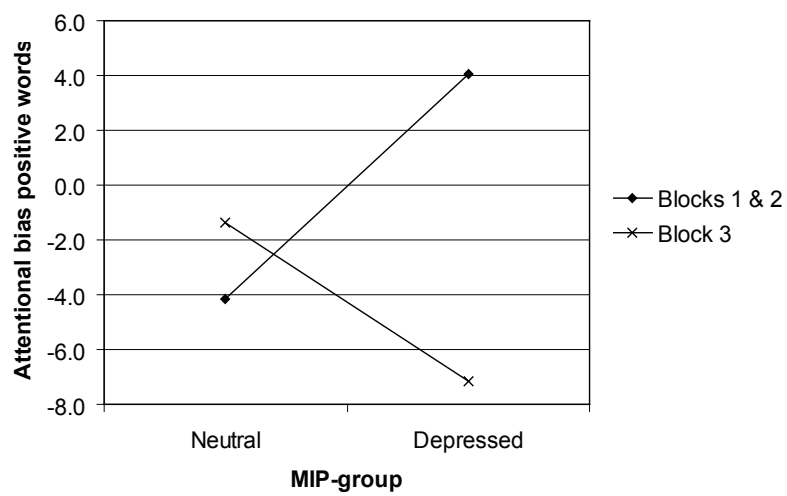

Figure 6.4 (a) ) The relation between BDI and RT bias scores for depression-relevant words in the second block. Participants from the neutral and depressed MIP group are indicated by different marker types. (b) RT bias scores for depression-relevant words for the long MIP-interval (i.e. block 2 and 4), for the neutral and the depressed MIP-group. (c) RT bias scores for positive words for the neutral and the depressed MIP-group. 
(i.e. block 2 versus block 4) $\times \operatorname{MIP}(F(1,90)=4.2, p=.04)$. As shown in Figure $6.4 \mathrm{~b}$, the depressed MIP-group in comparison with the neutral MIP-group showed a bias toward depression-relevant words in the first task-half (i.e. block 2) and a bias away from depression-relevant words in the second task-half (i.e. block 4). Separate analyses for the second and the fourth block showed that the difference between MIP-groups was significant only in the fourth block $(F(1,90)=4.9, p=.03$; second block: $F(1,90)=$ $1.1, p=.30)$.

Positive words. An effect of MIP-interval $(F(1,90)=4.2, p=.04)$ was superseded by an interaction of task-half $\times \operatorname{MIP}$-interval $\times \operatorname{MIP}(F(1,90)=4.3, p=.04)$. Therefore, again, analyses were done separately for every task-half and MIP-interval.

Analyses for every task-half (MIP-interval $\times$ MIP-group $\times$ BDI) only showed an effect in the first half (i.e. block 1 and 2). As shown in Figure 6.4c, different from what was hypothesized, participants in the depressed MIP-group, in comparison with participants from the neutral MIP-group, showed a bias towards positive words $(F(1,90)=4.6$, $p=.04)$.

Analyses for every MIP-interval only showed effects for the short interval. An overall bias away from positive words (intercept: $F(1,90)=4.6, p=.03$ ) was superseded by an interaction between task-half (i.e. block 1 versus block 3 ) and MIP-group $(F(1,90)=$ $8.1, p=.006)$. As shown in Figure $6.4 \mathrm{c}$, in the third block, following our hypotheses, the depressed MIP-group in comparison with the neutral MIP-group, showed a bias away from positive words. In the first block, the opposite effect was shown. Separate analyses for every block showed that the effect of MIP-group was significant only in the first block $(F(1,90)=6.1, p=.02$; third block: $F(1,90)=1.9, p=.17)$.

In sum, there were a number of effects of naturally-occurring dysphoria and of the negative mood induction on bias for depression-relevant and positive words. Notably, these effects were present only in some of the blocks. Furthermore, in none of the analyses there was an interaction effect of MIP-group and BDI. For depression-relevant words, consistent with our hypotheses, there was a positive correlation between BDI and bias (block 2). In addition there was an effect of mood-induced dysphoria (block 4) that differed in direction from what was hypothesized as the depressed MIP-group in comparison with the neutral MIP-group showed a bias away from depression-relevant words. For positive words, an effect of mood-induced dysphoria (block1 and block 2) was consistent with the effect for depression-relevant words as the depressed MIP-group in comparison with the neutral MIP-group showed a bias toward positive words.

Correlations between RT bias scores and questionnaire scores. To examine the relation between attentional bias scores and mood state, correlations were calculated between bias scores and questionnaire measures of the BDI, STAI-trait, STAIstate, and POMS scores obtained after the MIP. In addition to bias scores for positive words and depression words in each block, a summary bias score was computed for every participant in each block by subtracting the average bias score for positive words from the average bias score for depression words (Bradley, Mogg, \& Millar, 2000). This score reflects a preference for depression words relative to positive words. First, the results showed that measures of depression and state anxiety were positively related to the bias in the second block for depression-relevant words (BDI: $r=.20, p=.05$; POMS-depression: $r=.23, p=.03$; STAI-state: $r=.21, p=.04$; POMS-tension: $r=.30$, $p=.004)$, and to the bias in the second block for depression-relevant words relative 
to positive words (POMS-tension time 2: $r=.22, p=.03$ ). Second, trait anxiety was positively related to greater vigilance for depression words relative to positive words in the third block $(r=.22, p=.03)$. In sum, correlation analyses partly replicated results from the ANOVAs shown before, but also added some new results. First, measures of depression and state anxiety were positively related to biases in the second block for depression-relevant words, both relative to neutral and relative to positive words. Second, trait anxiety was related to biases in the third block, for depression-relevant words relative to positive words.

\section{Eye movements}

As shown in Figure 6.5a, participants with higher BDI scores tended to move their eyes away from emotional words in general, as reflected in a main effect of $\operatorname{BDI}(F(1,90)$ $=4.4, p=.04, r=-0.22$ ). In addition to this main effect, there were two interaction effects. Firstly, as shown in Figure 6.5b, the depressed MIP-group in comparison with the neutral MIP-group showed a bias away from depression-relevant words and toward positive words, as reflected by an interaction of MIP-group and wordtype $(F(1,90)=$ $6.4, p=.01)$. Separate analyses for every wordtype showed a difference between the two MIP-groups only for positive words $(F(1,90)=6.1, p=.02$; depression words $F(1$, $90)=1.5, p=.22)$. Secondly, an interaction of MIP-group and MIP-interval $(F(1,90)=$ $4.7, p=.03$ ) indicated a difference between the MIP-groups only for the long MIP-interval (i.e. block 2 and block 4; intercept: $F(1,90)=4.7, p=.03$; short MIP-interval: $F(1$, $90)=1.5, p=.23$ ). For the long interval, the main effect of MIP-group was superseded by an interaction of MIP-group and wordtype $(F(1,90)=5.6, p=.02)$, indicating an effect similar to Figure 6.5b.

In sum², the depressed MIP-group demonstrated a bias toward positive words and away from depression-relevant words in comparison with the neutral MIP-group. This difference was independent of BDI level. In addition to these effects, there was a relation between BDI and biases away from both types of emotional words. It should be noted that unlike RT results, eye movement bias results were consistent across task blocks.

ERPs. Results are reported separately for explicitly attention directing arrows and emotional-neutral word pairs. Figure 6.6a shows difference waves for arrow stimuli for each of the MIP-groups. These difference waves were calculated for right-hemisphere and left-hemisphere electrodes, by first subtracting the ERP signal for shifts to the right from the ERP signal for shifts to the left. Then, left-hemisphere difference waves were subtracted from corresponding right hemisphere difference-waves. Orienting-related contralateral negativity (positivity) is reflected in these waves by a negative (positive) amplitude. Whenever there was an effect of BDI, each MIP-group was divided into two groups based on a median split of the BDI (median BDI-score: 7).

\footnotetext{
2 Eye movement analyses were repeated on a sample of participants that had made more eye movements during the task than the rest. More specifically, participants were selected if they had a proportion of HEOG activity in the cue-target interval that was larger than $25 \%$ for both types of emotional words. The results of this subgroup ( $n=25$ for neutral MIPgroup, $\mathrm{n}=31$ for depressed MIP-group) were similar to the results of the whole sample.
} 
a

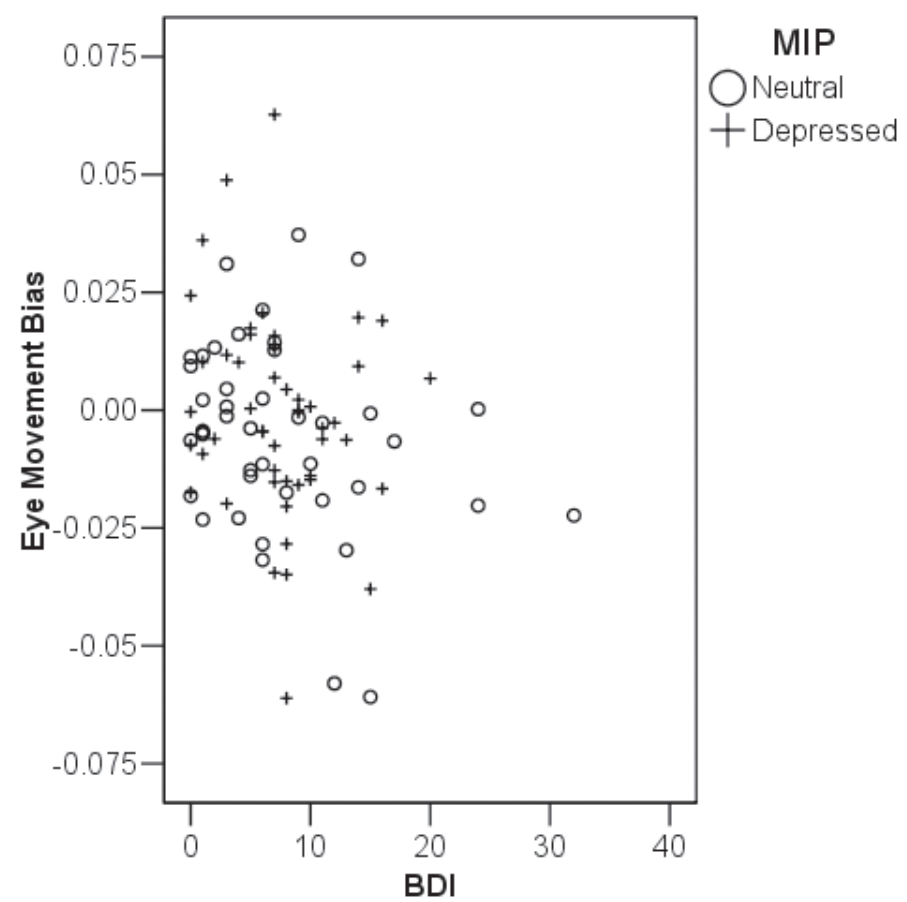

b

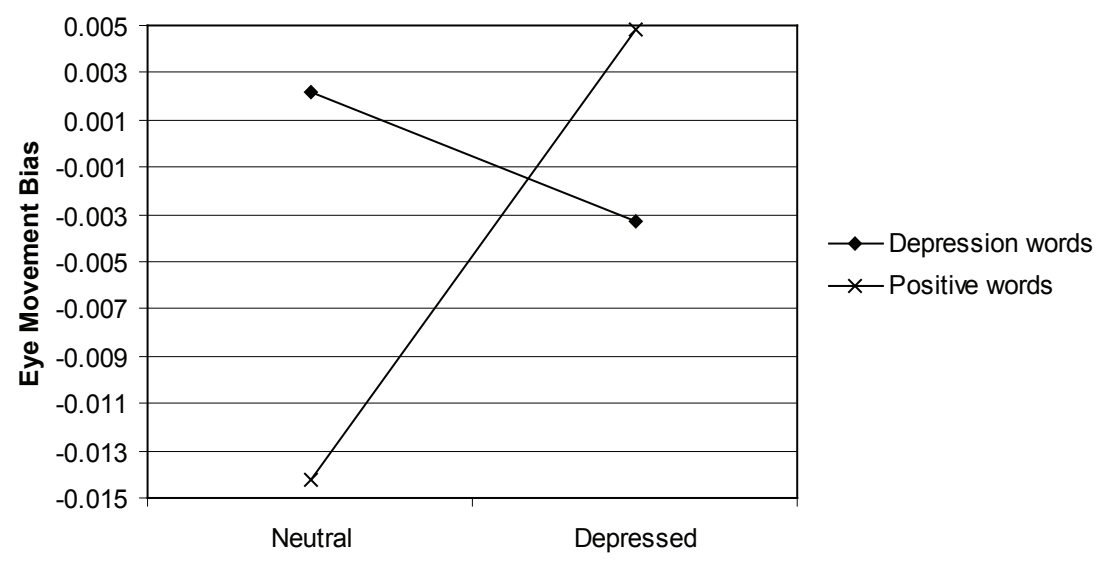

MIP-group

Figure 6.5 (a) The relation between BDI and eye movement bias for emotional words. Participants from the neutral and depressed MIP group are indicated by different marker types. Similar to RT bias scores, positive scores indicate a bias toward these words, negative scores indicate a bias away from these words. (b) Eye-movement biases for both emotional word types in the neutral and the depressed MIP-group. Electrophysiological graphs of the same data are presented on the next page. 


\section{8 chapter 6}

b, continued

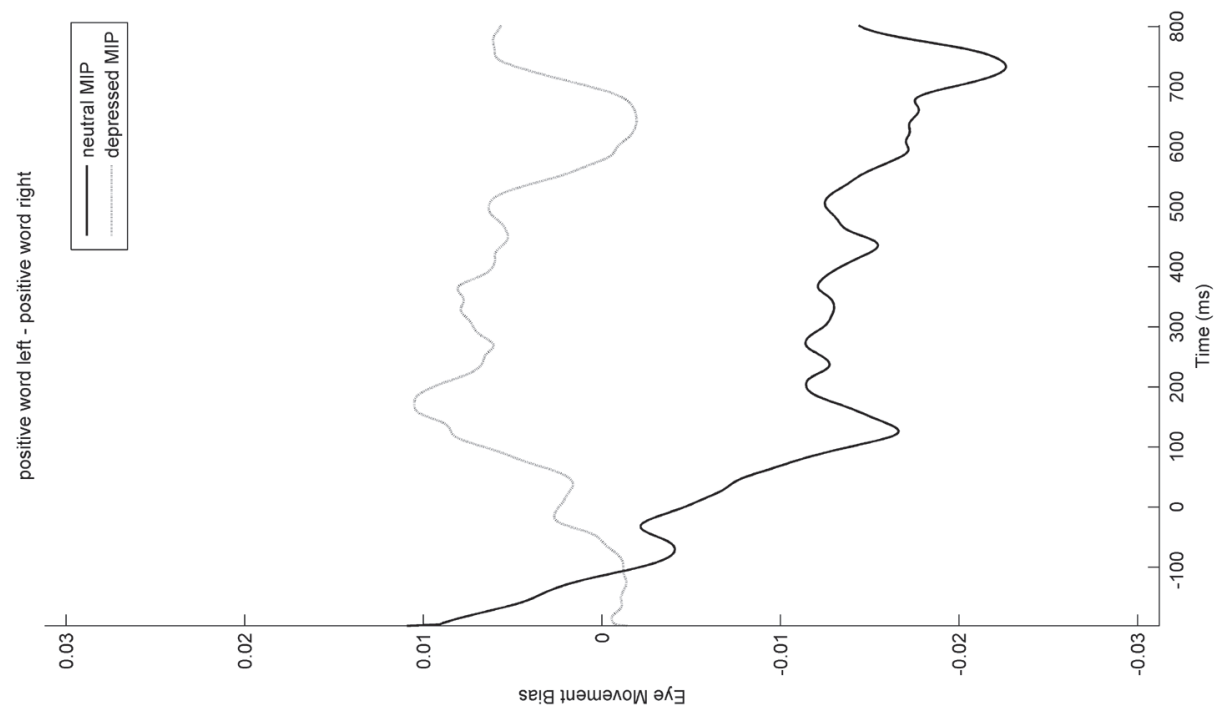

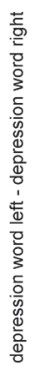

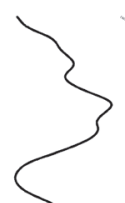

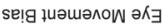


At posterior sites, between 150 and $300 \mathrm{~ms}$, a lateralized early positive component was indicated by an interaction between cue and hemisphere $(F(1,90)=31.7, p<$ .0005). This component has been found before (Jongen, Smulders, \& van Breukelen, 2006; Jongen, Smulders, \& van der Heiden, 2007) and likely reflects sensory processing of the arrow cues. This component was followed by a lateralized positive component between 300 and $500 \mathrm{~ms}(F(1,89)=32.0, p<.0005)$. The latter may reflect an early start of the LDAP shown between 500 and $800 \mathrm{~ms}(F(1,89)=78.0, p<.0005)$. The LDAP was significant at every electrode-pair, but strongest at $01 / 2$, as confirmed by an interaction of cue $\times$ hemisphere $\times$ electrode $(F(4,86)=3.7, p=.01)$. In addition there was an interaction of cue $\times$ hemisphere $\times \operatorname{BDI}(F(1,89)=4.9, p=.03)$ that was superseded by an interaction of cue $\times$ hemisphere $\times \operatorname{BDI} \times \operatorname{MIP}(F(1,89)=7.2, p=$ .009). When the difference between MIP-groups was examined separately for low-scorers and high-scorers on the BDI, it was shown that the LDAP was significantly smaller in the depressed MIP-group for the low-scorers on the BDI $(t(41)=2.1, p=.04)$. This is presented in Figure $6.6 \mathrm{~b}$. There was no difference between MIP-groups for the highscorers on the BDI $(t(48)<1, p=.68)$. RT-results have shown that although there was a RT validity effect for every group, costs were larger for the depressed MIP-group than for the neutral MIP-group. Therefore, any ERP difference between the MIP-groups might be related to this behavioral difference.

At frontal sites there was a lateralized positivity between 300 and $800 \mathrm{~ms}$ (300-500 ms: $F(1,89)=16.8, p<.0005 ; 500-800 \mathrm{~ms}: F(1,89)=9.9, p=.002)$. There was an interaction of cue, hemisphere and electrode $(300-500 \mathrm{~ms}: F(1,89)=22.3, p<.0005$; 500-800 ms: $F(1,89)=16.8, p<.0005)$ that indicated that this positive component was stronger at $F 7 / 8$ than at F3/4.

At central sites there was an interaction of cue, hemisphere and electrode between 300 and $500 \mathrm{~ms}(F(2,88)=8.1, p=.001)$. This indicated a positivity at $\mathrm{FC} / 6(F(1,89)$ $=5.5, p=.02)$ but no interaction of cue and hemisphere at $\mathrm{C} 3 / 4(F(1,89)<1, p=$ $.33)$ or $\mathrm{CP} 5 / 6(F(1,89)<1, p=.39)$. Between 500 and $800 \mathrm{~ms}$, there was an interaction between cue, hemisphere and electrode $(F(2,88)=9.9, p<.0005)$ that indicated a positivity at $\mathrm{FC} 5 / 6(F(1,89)=4.1, p=.05)$ and $\mathrm{CP} 5 / 6(F(1,89)=15.8, p<.0005)$, but not at $\mathrm{C} 3 / 4(F(1,89)<1, p=.48)$.

For depression-relevant and positive word stimuli there were no significant lateralized components.

In sum, a number of lateralized components were elicited by arrow stimuli. At posterior sites, arrow cues elicited an early positivity between 150 and $300 \mathrm{~ms}$. Second, at posterior sites between 300 and 500 ms another positive component was elicited that might reflect an early start of the LDAP that was elicited between 500 and $800 \mathrm{~ms}$. The latter was modulated by the interaction of MIP and BDI as it was smaller in the depressed MIP-group, but only in subjects that scored low on the BDI. This ERP difference between MIP-groups might be related to the smaller RT-costs in the neutral MIP-group than in the depressed MIP-group. At frontal and central sites, a contralateral negativity (ADAN) was expected as it has been shown in other studies. Instead, at frontal and at some of the central sites a positive component was shown between 300 and 800 ms. There were no lateralized effects at $\mathrm{C} 3 / 4$, which makes it unlikely that the effects at frontal and posterior sites result from the same neuronal source. Word stimuli did not elicit any lateralized activity. RT bias effects for emotional word stimuli strongly 
a
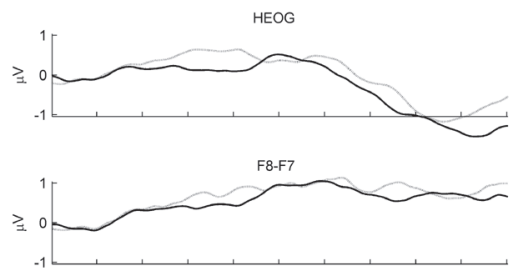

C4-C3
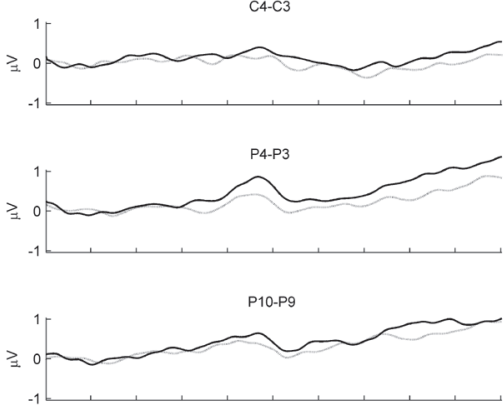

O2-01

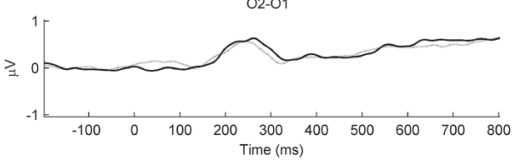

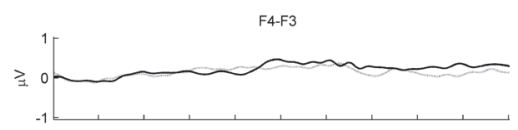

FC6-FC5
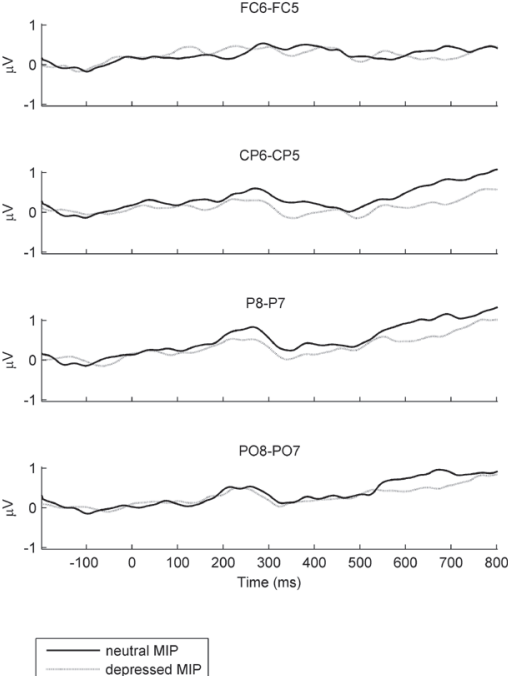

b
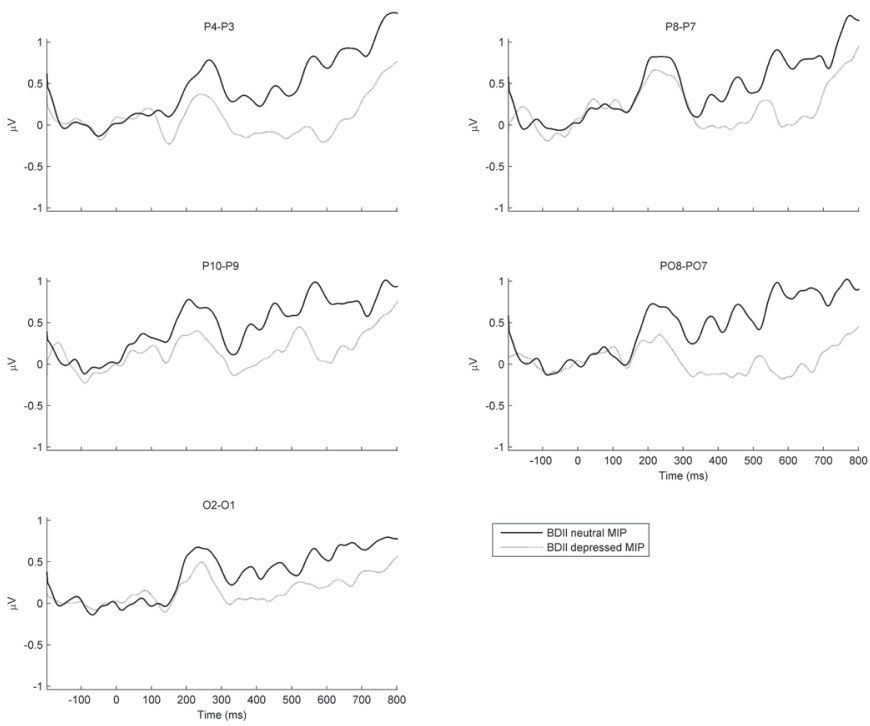

Figure 6.6 (a) Grand-averaged ERP difference waves at homologue electrode pairs in the cuetarget interval, in the arrow conditions, for the two MIP-groups. Negative components contralateral to the cued direction of attention are reflected by negative values, positive components are reflected by positive values. (b) Grand-averaged ERP difference waves for homologue electrode pairs at posterior sites in the cue-target interval, in the arrow conditions, for those participants in the neutral and the depressed MIP group that scored low on the BDI (BDII). 
depended on block. As a consequence, when all blocks were pooled, there were no significant RT bias effects. In that sense, RT bias effects and lateralized ERP effects were consistent with each other.

Event-related changes in the La1-band. Results are reported separately for arrows and emotional word stimuli. Difference waves were calculated in the same way as for ERPs. As a result, a contralateral decrease is reflected by negative amplitude values and a contralateral increase by positive amplitude values. Only posterior sites were examined (Jongen, Smulders, \& van Breukelen, 2006).

For arrow stimuli, as shown in Figure 6.7a, there was an overall contralateral increase in the La1-band between 0 and $200 \mathrm{~ms}(F(1,89)=4.3, p=.04)$. Between 400 and $600 \mathrm{~ms}$, lateralized activity differed between MIP-groups, depending on BDI-level, as indicated by an interaction of cue $\times$ hemisphere $\times \operatorname{MIP} \times \operatorname{BDI}(F(1,89)=4.5, p=$ $.04)$. Separate analyses for low-scorers and high-scorers on the BDI revealed a difference between the neutral MIP-group and the depressed MIP-group for low-scorers only $(t(41)=2.0, p=.05$; high-scorers: $t(48)<1, p=.65)$. As shown in Figure 6.7b, there was a contralateral increase of La1-activity in the neutral MIP-group and a contralateral decrease of La1-activity in the depressed MIP-group for participants that scored low on the BDI.

In sum, lateralized activity in the La1-band was elicited by arrow stimuli at posterior sites. A contralateral decrease in the (lower) alpha (1) band has been shown contralateral to stimuli that had to be attended (Babiloni et al., 2004; Jongen, Smulders, \& van Breukelen, 2006; Worden, Foxe, Wang, \& Simpson, 2000). Importantly, in both studies decreases related to attentional shifts started from 400-500 ms after cue-onset and lasted until target-onset. Earlier effects might reflect sensory effects of the cue instead, similar to the early lateralized ERP components described before. Here, after a very early contralateral increase, there was, between 400 and $600 \mathrm{~ms}$, a contralateral increase of activity in the neutral MIP-group and a contralateral decrease of activity in the depressed MIP-group, but only in subjects that scored low on the BDI. Note that in the ERP there was a similar modulation at posterior sites between 500 and $800 \mathrm{~ms}$. These differences between MIP-groups in the ERP and in lateralized La1-band activity might be related to the difference in RT costs between the two MIP-groups. A stronger contralateral decrease in La1-activity would in that case be related to stronger RT costs. Still, it should be noted that the difference in RT costs between the neutral and the depressed MIP-group was independent of BDI level. In addition, this effect was shortlasting.

Results for depression-relevant words are presented in Figure 6.8. As shown in Figure $6.8 \mathrm{~b}$, at posterior sites there was a large difference between low and high scorers on the BDI between 0 and $400 \mathrm{~ms}$. This was confirmed by statistics showing an interaction of cue $\times$ hemisphere $\times$ BDI $(0-200 \mathrm{~ms}: F(1,89)=9.1, p=.003 ; 200-400 \mathrm{~ms}$ : $F(1,89)=7.1, p=.01)$. Whereas low-scorers showed a contralateral decrease in the La1-band, high-scorers showed a contralateral increase in the La1-band. There was a positive correlation between BDI and contralateral activity in the La1-band between 0 and $400 \mathrm{~ms}(0-200 \mathrm{~ms}: r=.32 ; 200-400 \mathrm{~ms}: r=.22)$. Between 600 and $800 \mathrm{~ms}$, there was a significant five-way interaction $(F(4,86)=2.7, p=.05)$, indicating a difference between the two MIP-groups, but only for the low scorers on the BDI at P3/4 $(t(41)=$ $2.3, p=.03)$. As shown in Figure $6.8 \mathrm{c}$, there was a contralateral decrease of activity 
a
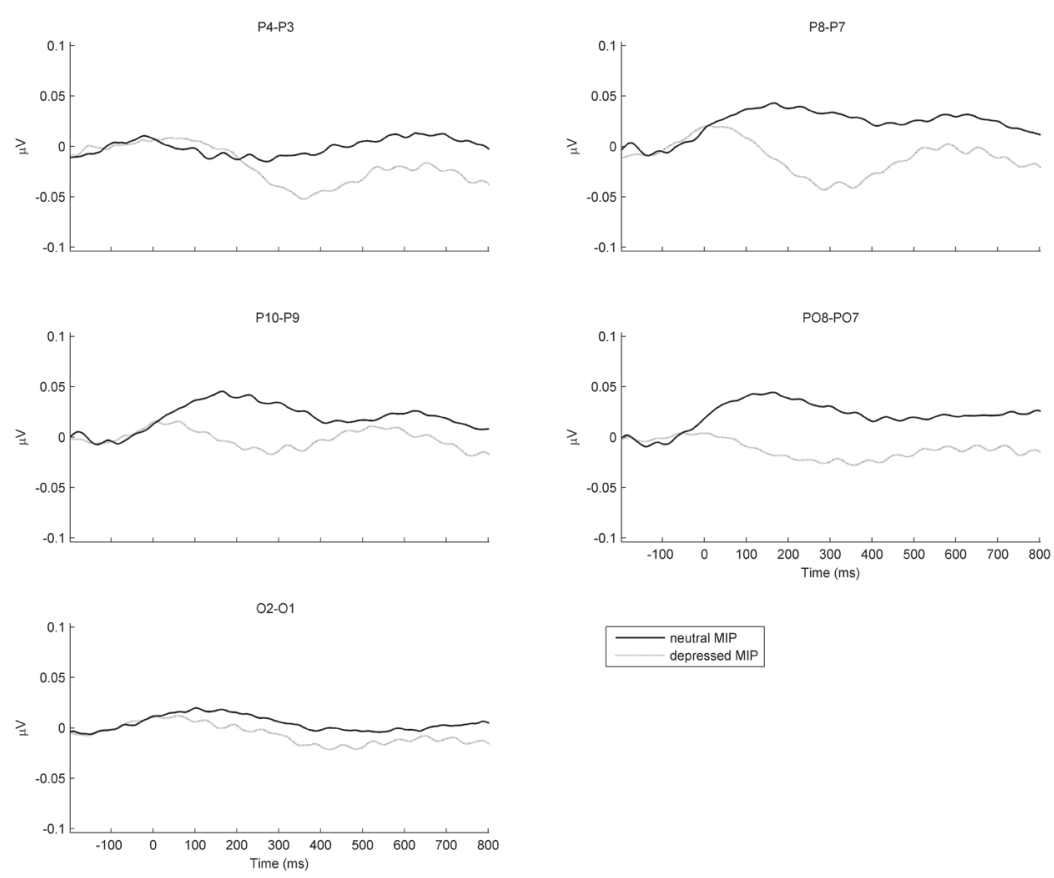

b
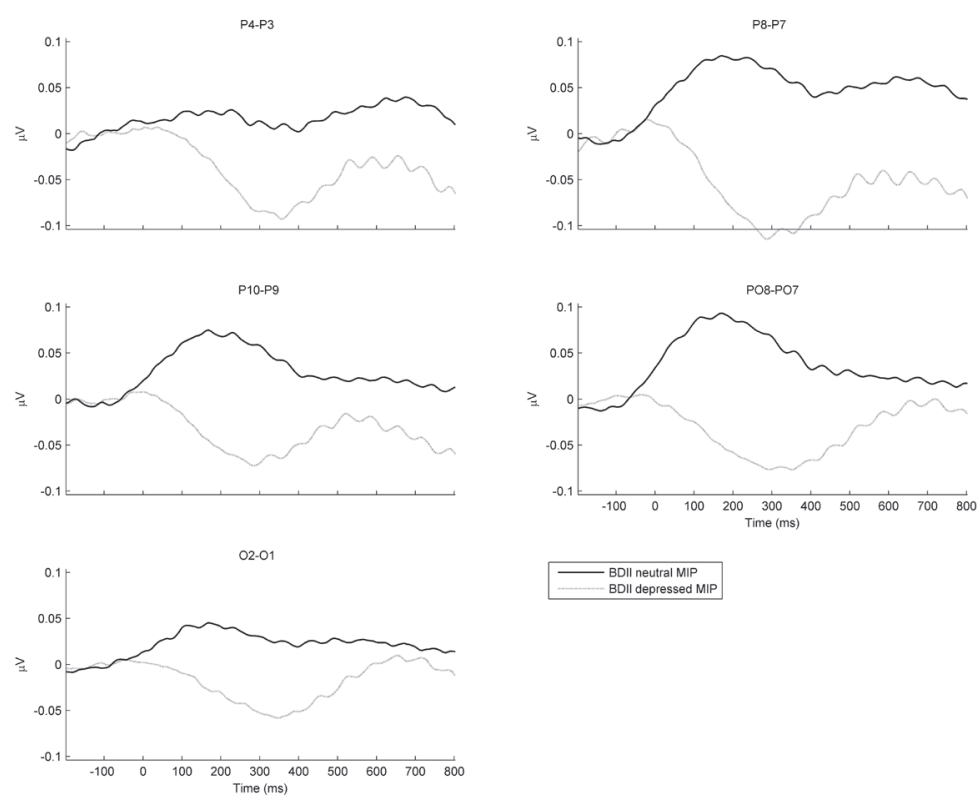

BDII neutral MIP

BDII depressed MIP

Figure 6.7 Grand averaged lower alpha 1 difference waves for posterior homologue electrode pairs in the cue target interval, in the arrow conditions. Decreases of lower alpha 1 contralateral to the direction of shifts of attention are reflected by a negative sign, increases are reflected by a positive sign. Results are presented (a) for the two MIP-groups, and (b) for those participants in the neutral and the depressed MIP group that scored low on the BDI (BDII). 
attentional bias and general orienting processes in dysphoria

a

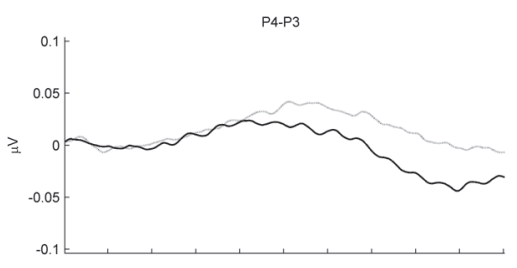

P10-P9

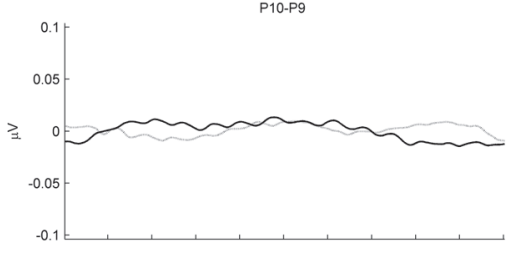

$02-01$

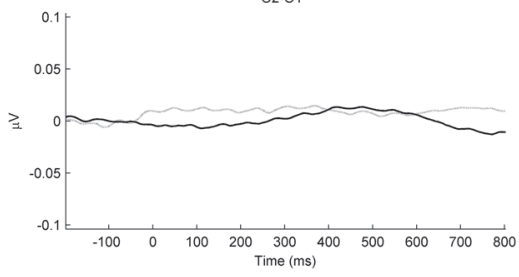

b
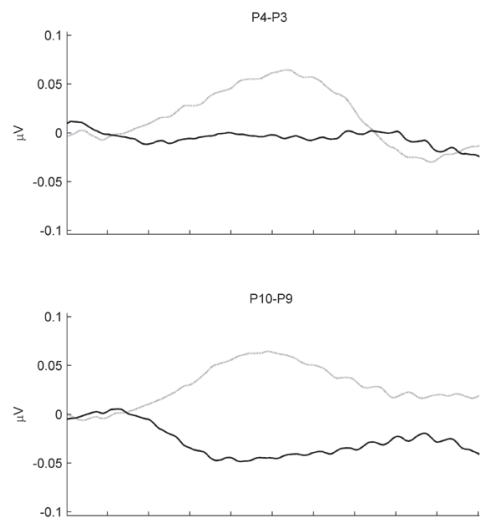

$02-01$

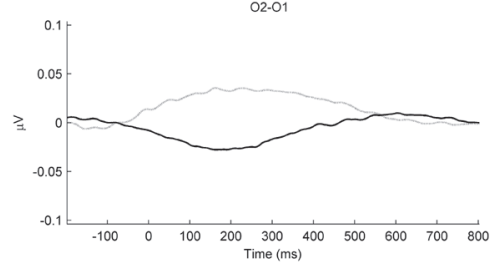

P8-P7

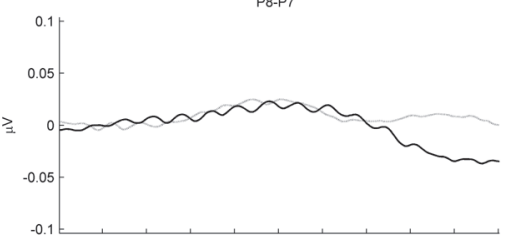

PO8-PO7
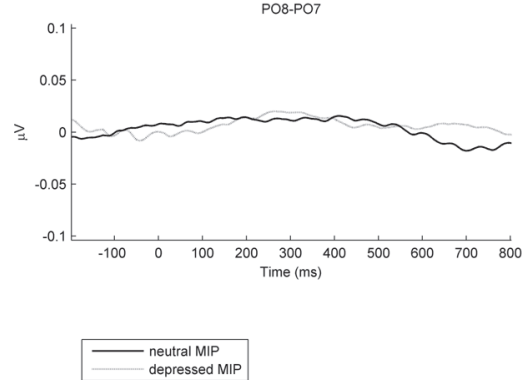

depressed MIP

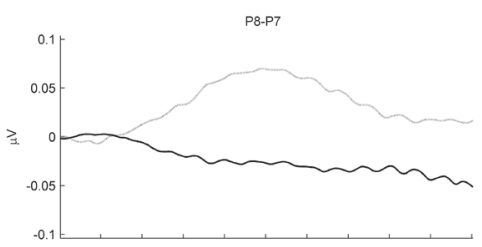

PO8-PO7
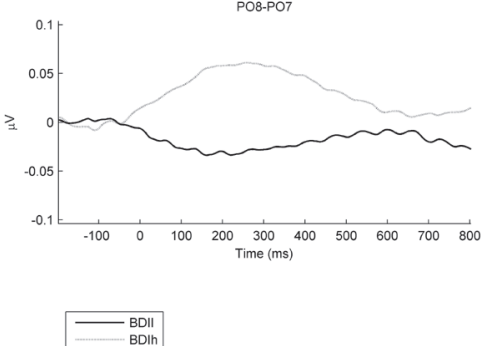
C
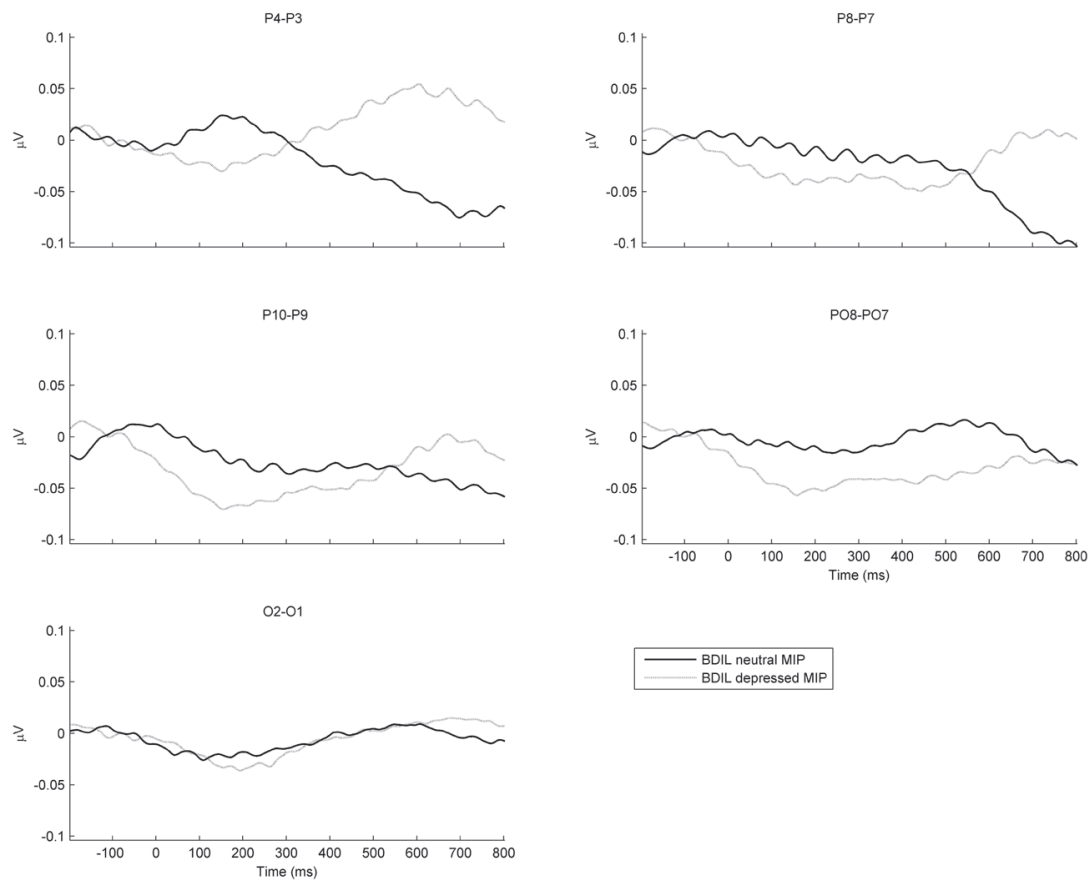

Figure 6.8 Grand averaged lower alpha 1 difference waves for posterior homologue electrode pairs in the cue target interval, for depression-relevant words. Decreases of lower alpha 1 contralateral to the direction of shifts of attention are reflected by a negative sign, increases are reflected by a positive sign. Results are presented (a) for the two MIP-groups; (b) for low-scorers (BDII) and high-scorers (BDIh) on the BDI; (c) for those participants in the neutral and the depressed MIP group that scored low on the BDI (BDII). 
in the La1-band in the neutral MIP-group, but a contralateral increase of activity in the La1-band in the depressed MIP-group.

Results for positive words are presented in Figure 6.9. There was an interaction of cue $\times$ hemisphere $\times$ BDI $\times$ MIP between 0 and $200 \mathrm{~ms}(F(1,89)=5.6, p=.02)$. This interaction was a reflection of a negative correlation between BDI and contralateral activity in the La1-band in the depressed MIP-group $(r=-.30, p=.04)$, and no effect of BDI in the neutral MIP-group $(F(1,41)<1, p=.38)$. This effect is shown in Figure $6.9 \mathrm{~b}$ where the depressed MIP-group was split into groups of low-scorers and high-scorers on the BDI. Between 400 and $600 \mathrm{~ms}$, there was a five-way interaction $(F(4,86)=3.8$, $p=.02)$, indicating an interaction of cue $\times$ hemisphere $\times \mathrm{BDI} \times \mathrm{MIP}$ at $\mathrm{P} 3 / 4(F(1,89)$ $=4.2, p=.04)$. Again, there was an effect of BDI only for the depressed MIP-group ( $F(1,48)=4.6, p=.04$; neutral MIP-group: $F(1,41)<1, p=.66)$ and correlation analyses showed a negative correlation between BDI and contralateral activity in the La1-band $(r=-.30)$.

In sum, there were lateralized changes in La1-band activity at posterior sites after the presentation of emotional word stimuli. Between 0 and $400 \mathrm{~ms}$, after the presentation of depression-relevant words, there was a large difference between low and high scorers on the BDI. Whereas low-scorers showed a contralateral decrease of activity in the La1-band, high-scorers showed a contralateral increase of activity in the La1-band, and correlation analyses showed a positive correlation between BDI and contralateral La1-activity. There was a negative correlation between BDI and contralateral La1-activity for positive words in the depressed MIP-group between 0 and $200 \mathrm{~ms}$ and between 400 and $600 \mathrm{~ms}$. Finally, for depression-relevant words there also was a difference between MIP-groups that occurred later in time $(600-800 \mathrm{~ms})$ but was limited to low-scorers on the BDI. According to the interpretation of lateralized La1-activity that was given before, the modulatory effect of BDI on La1-band activity that is opposite in direction for depression-relevant words and positive words is too early to interpret it in terms of orienting effects. However, after $400 \mathrm{~ms}$ two effects were shown. For depression-relevant words, there was a difference between MIP-groups in the group of low-scorers on the BDI as participants from the depressed MIP-group in comparison with the neutral MIPgroup directed attention away from depression-relevant words. For positive words, a correlation of BDI-scores in the depressed MIP-group showed that high-scorers tended to direct their attention toward positive words. As for arrow stimuli effects again were short-lasting. In addition they were limited to one electrode-pair.

\section{Discussion}

The aims of the present study were to investigate general orienting processes and attentional bias in induced and naturally-occurring dysphoria, and to measure the time course of these processes. Participants were assigned to a neutral MIP-group or a depressed MIP-group. Subjective measures all indicated that the MIP was successful in inducing a negative mood. EMs and EEG were registered while participants performed on an adjusted version of the dot-probe task with a spatial cueing paradigm incorporated in it. 
a
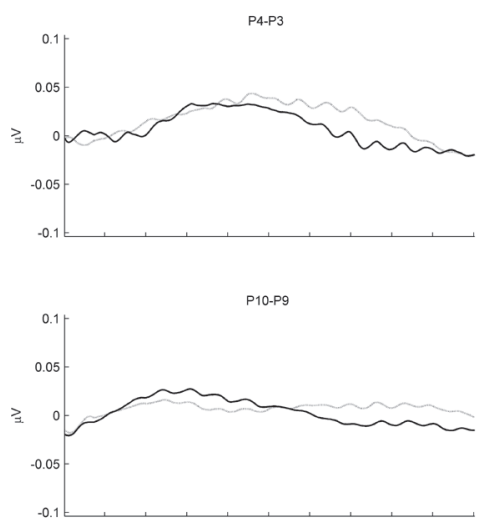

$02-01$

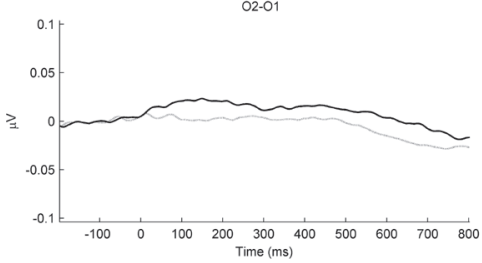

b
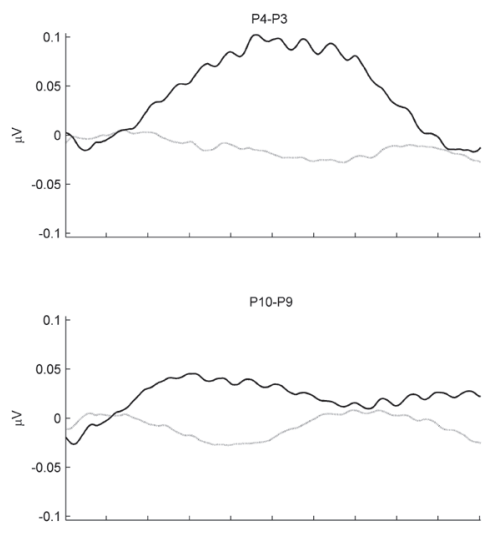

$02-01$

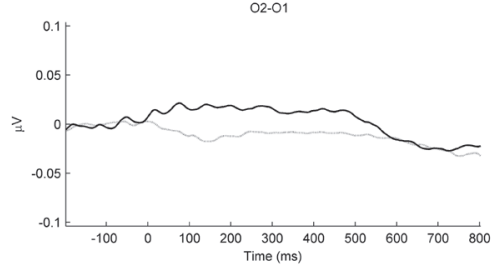

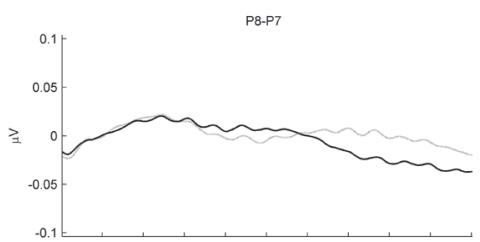

PO8-PO7
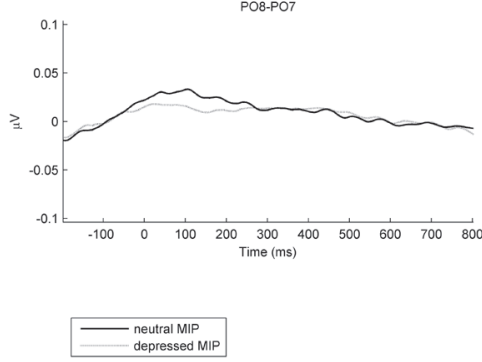

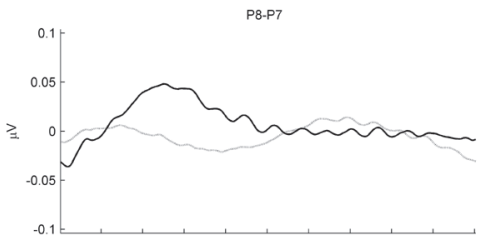

PO8-PO7

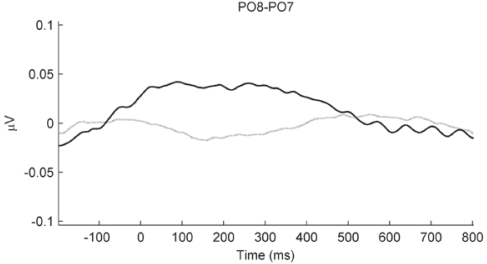

\begin{tabular}{|r|}
\hline BDIL depressed MIP \\
BDIH depressed MIP
\end{tabular}

Figure 6.9 Grand averaged lower alpha 1 difference waves for posterior homologue electrode pairs in the cue target interval, for positive words. Decreases of lower alpha 1 contralateral to the direction of shifts of attention are reflected by a negative sign, increases are reflected by a positive sign. Results are presented (a) for the two MIP-groups; (b) for low-scorers and high-scorers on the BDI from the depressed MIP-group. 


\section{General orienting processes: arrow stimuli.}

Table 6.5 shows a summary of the results of all dependent measures for general orienting processes. A discussion for every dependent measure is followed by an integration of the results from all measures.

Reaction time results. Although all participants oriented their attention in response to arrow cues, as demonstrated by significant benefits, substantial costs of attention were only observed in the depressed MIP-group indicating a problem in disengaging attention from a previously cued location. A comparable interplay of mood and attentional processes has been shown in a sample of patients with bipolar disorder who performed on the same task presented here (Jongen, Smulders, Ranson, Arts, \& Krabbendam, in press). It was shown that depressed mood (measured with the BDI) was positively related to the amount of costs. Importantly, in the present study costs were independent of naturally-occurring dysphoria, measured with the BDI. This difference is probably due to a more depressed mood, reflected by higher BDI-scores, in the patient group than in the dysphoric group that was tested here. The effect is also similar to the effect shown by Compton (2000) that was mentioned in the introduction. Bradley et al. (1997) suggested that disengagement problems may be the underlying mechanism for attentional bias in depression and dysphoria. Some evidence for this was shown in two studies that used a cueing paradigm with single word cues (Ellenbogen, Schwartzman, Stewart, \& Walker, 2002; Koster et al., 2005). In one of these, dysphorics were slow to disengage attention from negative, positive, and neutral words following a negative MIP (Ellenbogen et al., 2002). Koster et al. (2005), showed that dysphorics had difficulty to disengage attention from negative words. The present results suggest that disengagement problems are not specific for negative words. Instead dysphoria and depression may be characterized by a core deficit of disengagement. If attention is preferentially oriented towards negative words because of word valence, this may result in problems of disengagement similar to those that were shown here when attention was directed by arrow cues.

In sum, the current results showed that lowered mood was related to a relative inability to disengage attention from nonemotional information when it was directed by arrow cues. For future studies it will be important to determine whether this general inability to disengage attention is also characteristic of unipolar depression as, in a visual search task with schematic faces (neutral, positive, negative), there was no evidence for disengagement problems in a group of major depressed participants (Karparova, Kersting, \& Suslow, 2005). Furthermore, replication of these results is necessary.

ERP results. A number of lateralized components were elicited by the arrow stimuli. The earliest component, a posterior positivity between 150 and $300 \mathrm{~ms}$, has repeatedly been found when similar arrow cues were used and almost certainly reflects sensory processing of the cue (Jongen, Smulders, \& van Breukelen, 2006; Jongen, Smulders, van der Heiden, 2007; Verleger, Vollmer, Wauschkuhn, van der Lubbe, \& Wascher, 2000). Following this early component, at posterior sites a contralateral negativity, the EDAN, was expected. Instead, between 300 and $500 \mathrm{~ms}$ another contralateral positive component was elicited. For now, we can only speculate why no EDAN was found. First, an EDAN was expected here because similar arrow stimuli and the same task instruction (detection task) were used in two of our previous studies where an EDAN was found (Jongen, Smulders, \& van Breukelen, 2006; Jongen, Smulders, \& van der Heiden, 2007, 


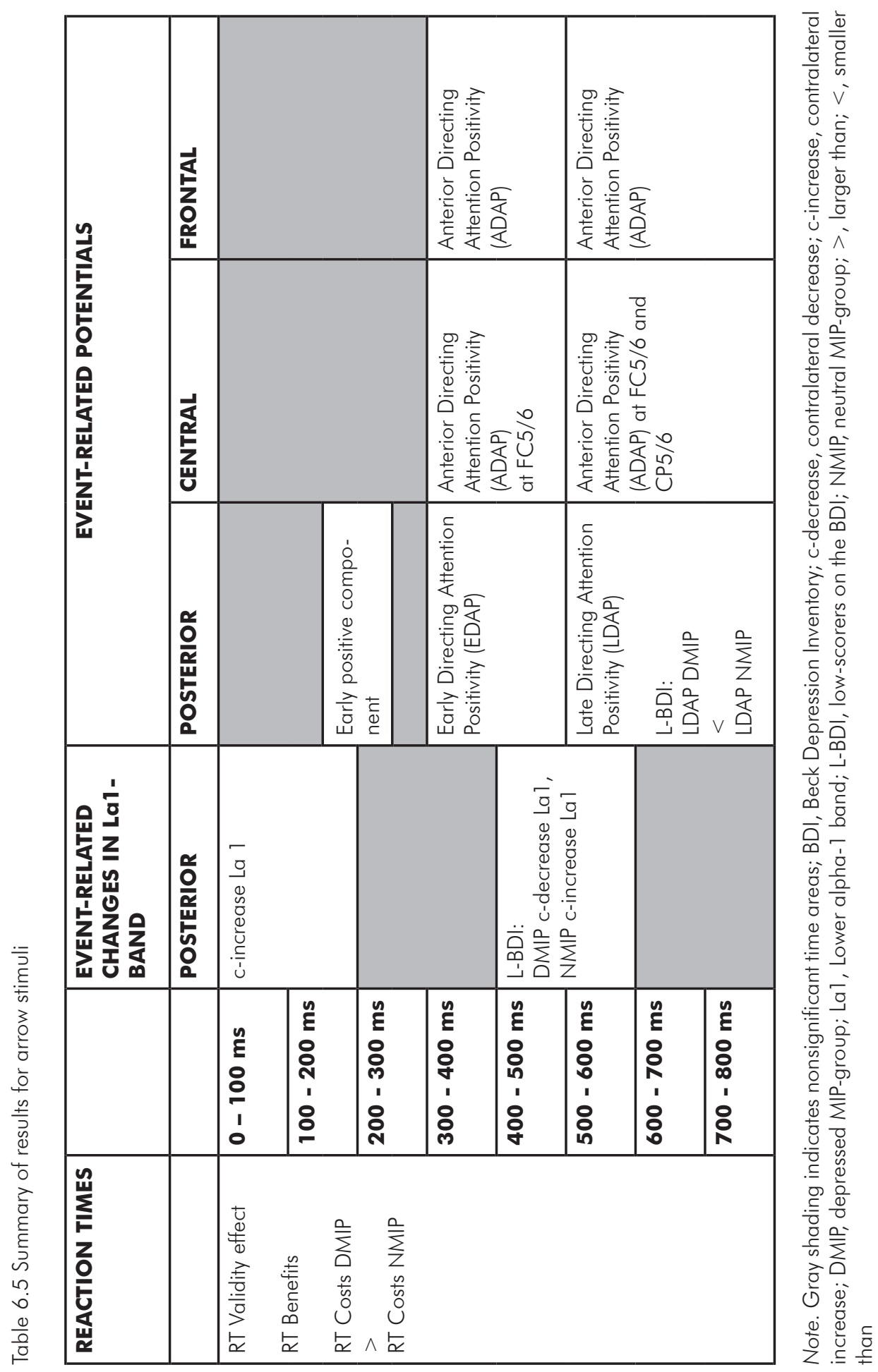


experiment 1). However, the similarity only concerns the Posner cueing paradigm of the present task. A difference is that in the present task a word pair was presented along with every arrow. Although these words were always both neutral and neutral words were not expected to draw or hold attention, it cannot be excluded that the words are responsible for the lateralized positivity. Another explanation follows from the second experiment in a study from our lab (Jongen, Smulders, \& van der Heiden, 2007) where a lateralized positivity was also found between 300 and $500 \mathrm{~ms}$. Those data suggested a spatial and temporal overlap of the EDAN and the LDAP. That is, the LDAP might always have an early start, around 300 ms after cue onset, but be cancelled out when an EDAN is stronger. For the present results this would mean that the EDAN was smaller than the LDAP. However, other techniques (e.g. source localization of each component) are necessary to confirm this interpretation.

Finally, at posterior sites, an LDAP was elicited between 500 and $800 \mathrm{~ms}$. The LDAP has been assumed to reflect modulation of the cortical excitability in those regions that process the upcoming visual information (Harter et al., 1989). However, as noted in the introduction some studies have provided results that are inconsistent with this assumption. Here, interestingly, the LDAP was modulated by an interaction of MIP-group and BDI as it was smaller in the depressed MIP-group, in subjects that scored low on the BDI. This ERP difference between the two MIP-groups might be related to the difference in RT costs between the two MIP-groups. However, this would implicate that larger costs are related to a decrease of the LDAP. In terms of cortical excitability, the opposite would have been expected. In addition, it should be noted that the difference in RT-costs was not limited to the low-scorers on the BDI but was independent of BDI score. Therefore, these data on the LDAP at this moment do not allow for strong conclusions.

At frontal and central sites based on other studies, a contralateral negativity (ADAN), associated with attentional control, was expected. Instead, at frontal and at some of the central sites a positive component was shown between 300 and $800 \mathrm{~ms}$. There were no lateralized effects at $\mathrm{C} 3 / 4$, which makes it unlikely that the effects at frontal and posterior sites result from the same neuronal source.These components were not modulated by any group differences. Again (as for the posterior positivity) we can only speculate that differences between previous cueing tasks and the present combination of a spatial cueing task and dot-probe task are responsible for the difference in ERP components between these tasks. Replication is therefore necessary.

Event-related changes in the La1-band. As all groups showed a RT validity effect, from previous findings a lateralized decrease in the La1-band at posterior sites, starting around $400 \mathrm{~ms}$ after cue onset, was expected. Between 400 and $600 \mathrm{~ms}$ after cue onset, for low-scorers on the BDI, there was a lateralized decrease in La1-band in the depressed MIP-group, but a lateralized increase in the neutral MIP-group. Similar to the modulation of the LDAP, this modulation may be related to the MIP-group difference in RT costs. A stronger contralateral decrease in La1-activity would in that case be related to stronger RT costs. As a contralateral decrease of La1-activity is related to an increase of cortical excitability, this effect is in the expected direction. However, it should be noted again that the difference in RT costs between the neutral and the depressed MIP-group was independent of BDI level. In addition, this effect was shortlasting in comparison with other studies (Jongen, Smulders, \& van Breukelen, 2006; 
Worden, Foxe, Wang, \& Simpson, 2000). It will be important for future studies to further examine this relation of lateralized activity in the La1-band and RT costs.

In sum: general orienting capacity. RT results showed that all participants were able to orient their attention. Importantly, an induced negative mood led to a relative inability to disengage attention. In line with this, Dreisbach and Goschke (2004; Dreisbach, 2006) have shown that induced positive affect in comparison with neutral or negative affect was related to an increase in cognitive flexibility that is necessary for example to deal with unexpected events. An invalidly cued target, having an .20 probability, may be regarded an unexpected event. Therefore, larger costs in the depressed MIP-group are in line with the idea of reduced cognitive flexibility when mood is lowered. ERP results partly replicated previous studies with lateralized components at posterior sites. Results of lateralized activity in the La1 band were minimal in comparison with previous studies. A group difference was shown for lateralized ERP activity and lateralized changes in the La1-band at posterior sites that might be related to the group difference in costs. More specifically, for the depressed MIP-group in comparison with the neutral MIP-group, the LDAP was smaller and there was a larger lateralized decrease in the La1-band.

\section{Attentional bias for emotional word stimuli.}

Table 6.6 shows a summary of the results of all dependent measures for attentional bias results. Again, a discussion of results for every dependent measure is followed by an integration of all findings.

Reaction Time Results. The number of trials that were used in the present study to examine attentional bias was larger than in most published dot-probe tasks. This was done to improve the signal-noise ratio of the EEG. The factor "task-block" was entered in the analyses as within-subjects variable (in a 2-by-2 design: task-half $\times$ MIP-interval). Unexpectedly, there were large differences in biases between the different task blocks. However, other dot-probe studies also have demonstrated that biases sometimes vary between blocks (e.g. Bradley et al., 1999). Liu et al. (2006) aimed at studying the time course of attentional bias in non-clinical anxiety and therefore presented four blocks of trials, each consisting of 72 trials. In line with our data their results showed that attentional bias was not stable over time. There were differences between low and high trait anxious only in the second and the third block. Another recent attempt to examine variance in bias results over time comes from a dot-probe study by Schmukle (2005) who examined internal consistency and test-retest reliability with one week in between two tests. There was no evidence for internal consistency or stability over time. Similarly, Jansson and Lundh (2006) found low test-retest reliability for biases measured on a Stroop task. In sum, these studies challenge the notion that biased attention has the stability that would be expected if it were a trait characteristic.

When the variance of bias effects over time is ignored and bias RT results for depression-relevant words and positive words are examined (see Table 6.6), two main findings can be discriminated; one related to naturally-occurring dysphoria, and one related to the induced negative mood. For naturally-occurring dysphoria, results were in line with the mood-congruency hypotheses, showing that dysphoria and anxiety were positively related to a bias for depression-relevant words. This result was absent for positive words, providing support for the valence hypothesis (cf. Mogg et al., 1995). Bradley et 


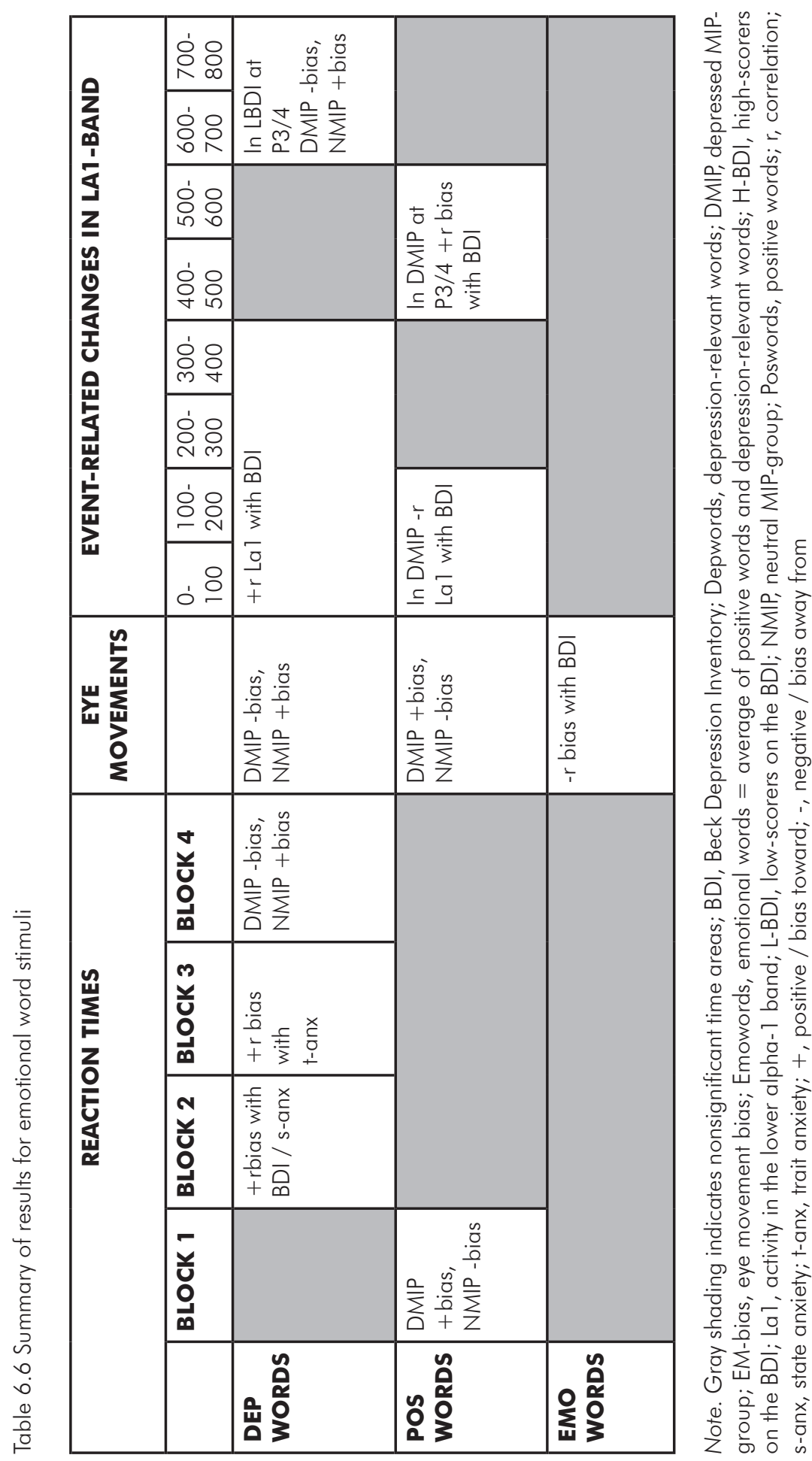


al. (1997, experiment 2) also reported a positive correlation between bias scores and BDI when words were presented for $1000 \mathrm{~ms}$. For induced dysphoria, the bias pattern for the two MIP-groups for depression-relevant words was the opposite of the bias pattern for positive words. In the depressed MIP-group in comparison with the neutral MIP-group, there was a bias away from depression-relevant words and a bias toward positive words. This patterns opposes the mood-congruency hypothesis, and results of Bradley et al. (1997, experiment 1). In their study, a depressed MIP-group showed a mood-congruent bias for depression-relevant words (no positive words were included). However, it should be noted that when variance over time is taken into account, the group difference for depression-relevant words in our task was demonstrated only in the fourth block, and the effect presented by Bradley et al. occurred in the first two blocks. Therefore, hypothetically this effect might have changed if more block repetitions had been used by Bradley et al. Furthermore, a pattern opposing mood-congruency, like we obtained, has also been shown by others (Ellenbogen, Schwartzman, Stewart, \& Walker, 2002; Miranda, Gross, Persons, Hahn, 1998). After a negative mood induction, participants were faster in disengaging attention from negative stimuli than from positive or neutral stimuli in comparison to a neutral or a positive induction (Ellenbogen et al., 2002). It was reasoned that rapid disengagement from negative words may be a reflection of an adaptive mechanism to regulate emotional arousal. The same mechanism probably underlies the bias away from depression-relevant words that was found here. Miranda et al. (1998) showed that after a negative mood induction there was an increase of negative thinking in those vulnerable for depression, but a decrease in participants without a history of depression. They reasoned that, as proposed by others (Gross \& Munoz, 1995), emotion regulatory processes may minimize the impact of negative mood shifts. These regulatory processes may act as coping mechanisms and may distinguish people that are vulnerable to depression from people that are not vulnerable to depression. In line with this, it has been proposed that the abilities to efficiently disengage and shift attention represent critical dimensions of self-regulatory behavior and effective coping (Derryberry \& Rothbart, 1997; Derryberry \& Reed, 2002). Derryberry and Reed (2002) showed that trait-anxious individuals with good attentional control were able to reduce their threat-related attentional bias and concluded that the magnitude and time course of attentional biases may always depend on the capacity to control attention. Finally, a pattern of avoidance from negative stimuli has also been shown in anxiety research when presentation duration of stimuli was long enough to allow strategic processing (e.g. Mogg, Bradley, Miles, \& Dixon, 2004; Rohner, 2002). Again this has been suggested to function as a way to reduce state anxiety (Mogg et al., 2004).

In sum, RT results in the dot-probe task showed a mood-congruent bias toward depression-relevant words that was related to measures of dysphoria and anxiety. Second, the attentional bias away from depression-relevant words in the depressed MIP-group in comparison with the neutral MIP-group may reflect an adaptive mechanism to regulate emotion. On the spatial cueing trials, a relative inability to disengage attention from neutral words was shown for the depressed MIP-group. The attentional bias away from depression-relevant words might similarly be a reflection of problems to disengage attention from neutral words. As noted by others (Koster, Leyman, de Raedt, \& 
Crombez, 2006) for future studies on depression and dysphoria it will be important to examine the relation between attentional bias and measures of attentional control. In addition, history of depression should always be rated to determine if a bias away from negative stimuli is specific for those non-vulnerable for depression, as suggested by Miranda et al. (1998). Third, bias results were unstable over time. Although it is not clear what caused this variance, it may in itself be an important reason for the heterogeneous findings of attentional bias in dysphoria and depression. It is clear from Table 6.6 that if we only had measured attentional bias by using one task block, there would have been no evidence for an attentional bias for depression-relevant words in dysphoria. In addition, our results underscore the importance of including the factor block as a within-subjects factor in a statistical design if stimuli have been presented repeatedly. If blocks had been pooled in our analyses, no significant differences between groups or related to naturally-occurring dysphoria had been found.

Eye-movements. It is important to note that (unlike RT biases) the pattern of EM biases was stable across task blocks. Again, there were two main findings; one related to naturally-occurring mood, and one related to the induced negative mood (see Table 6.6). Naturally-occurring dysphoria was related to an EM-bias away from positive and depression-relevant words. Therefore, EM results are in line with the emotionality hypothesis that predicts similar biases for different kinds of emotional valence (cf. Martin, Williams, \& Clark, 1991). Thus, for naturally-occurring dysphoria, the pattern of EMbiases was different from the patern of RT biases. Since EMs in time occurred before participants responded to the dot, it can be concluded that an (overt) bias away from all emotional words was followed in time by a (covert) bias toward depression-relevant words. This was what happened in the second and the third task block. In the first and the last task block, dysphoria was only related to the overt bias away from all emotional words. Similarly, in a dot-probe study (Mogg, Millar, and Bradley, 2000) an EM-bias toward threat in anxious participants was not followed by a RT-bias. It was reasoned that there were biases in initial orienting, but attention was not maintained at threat stimuli until the probe appeared. For the present results, an initial orienting response away from emotional stimuli probably was followed by a response back to fixation in the first and the last task block, and a response toward depression-relevant words in the second and the third block. This shows the importance of tapping attention at different points in time. Although highly speculative, the avoidance response away from all emotional stimuli may serve to regulate and attenuate emotional arousal in those scoring higher on dysphoria. For induced dysphoria, the pattern of EMs was similar to the RT result, as the depressed MIP-group in comparison with the neutral MIP-group showed a bias away from depression-relevant words and a bias toward positive words. This suggests that attention was maintained after the initial orienting response and explanations for this pattern of biases in terms of emotion regulation were already given when the RT bias pattern was discussed.

In sum, evidence for early attentional biases in dysphoria was shown for the first time. EM bias scores partly were similar to RT bias scores and partly were complementary. Naturally-occurring dysphoria was related to a bias away from all emotional stimuli, and EM bias results for induced dysphoria were similar to RT bias results. This is the first dot-probe study with measurement of EMs in dysphoria. Other studies have shown no evidence for biases in dysphoria or depression when brief, masked stimulus 
exposures were used and RT biases were measured (e.g. Bradley et al., 1997; Mogg et al., 1995; Mathews et al., 1996). This leads to the suggestion that EMs provide a more sensitive measure of attentional bias. Alternatively, EM-biases may be functionally different from biases measured with RT. However, as noted in the introduction, it is thought that EMs are guided by covert attention. Because this is the first time early attentional biases were shown and EMs were measured in a dot-probe task in naturallyoccurring and induced dysphoria, it will be important for future studies to provide replication. However, these results show the importance of tapping attention at different points in time. As suggested by others (Mogg et al., 2000), the distinction between initial orienting and maintenance of attention may be important in contributing to the heterogeneous findings across studies.

ERP results. To examine ERP results, it was again necessary to average the results from the different task blocks because signal-noise ratio in separate blocks would be low. There was no lateralized ERP response to word onset. We can only speculate about the reason for this absence, because this was the first study that has examined lateralized activity after word onset to study attentional bias. RT bias effects for emotional word stimuli strongly depended on task block, and when all blocks were pooled there were no significant RT bias effects. The absence of lateralized ERP effects might be the result of a similar instability. However, another possibility for the absence of the expected N2pc component may be the instructed irrelevance of word stimuli in a dotprobe task. In studies that reported an N2pc the stimuli that, when selected, elicited an N2pc were always relevant for task performance. In line with the component's functional definition it has been shown that the component was smaller when the need for focused attention was lower (Luck, Girelli, McDermott, \& Ford, 1997), for example when a search array only contained a single item in each hemifield. The only exception we are aware of regarding the relevance of stimuli is a study by Girelli and Luck (1997) in which motion singletons served as non-relevant distracter stimuli that still caused an N2pc. However, it was concluded that motion probably attracts attention in an automatic way.

In sum, there was no lateralized ERP activity elicited time-locked to word onset. Possibly, this is an effect of pooling unstable results across blocks, or the instructed irrelevance of the word stimuli.

Event-related changes in the La1-band. Results from the different task blocks were again averaged. Lateralized decreases in the La1-band at posterior sites, starting around $400 \mathrm{~ms}$ after word onset, might indicate effects of attentional orienting. There were two findings, one for induced dysphoria and one for naturally-occurring dysphoria. The effect for induced dysphoria, although limited to depression-relevant words, was similar to the pattern of EM-biases and RT-biases: the depressed MIP-group in comparison with the neutral MIP-group showed a bias away from depression-relevant words. However, here the effect was limited to the low scorers on the BDI, was only shown at one electrode-pair, and was short-lasting. For naturally-occurring dysphoria, the effect differed from the avoidance response that was shown for EM-biases, as there was a positive relation between biases for positive words and dysphoria. However, here the relation was limited to the depressed MIP-group. In addition it was limited again to one electrode pair, and short-lasting. All this indicates that the effects in the La1-band were weaker than the strong pattern of biases shown in the EM data. In addition data 
for arrow stimuli were also weaker than in previous studies. It therefore is probably best to refrain from drawing strong conclusions at this moment based on the data from the La1-band. Below we attempt to integrate results from all dependent measures.

In sum: attentional bias in naturally-occurring and induced dysphoria. All measures of attention that were used in the present study to examine the role of attentional bias in dysphoria may have certain shortcomings. Therefore, integrating the results of the different measures and focussing on converging results is important. Since RTs measured attention at another, later point in time than EMs and EEG, the time course of attention can be described. However, there were no significant lateralized ERP components in response to word stimuli and effects of lateralized activity in the La1 band were only weak. Therefore we will focus on findings from EMs. For naturally-occurring dysphoria, EMs showed a negative relation between dysphoria and the bias for depression-relevant words. That is, increases of dysphoria were related to an attentional bias away from depression-relevant words. RTs showed a positive relation between negative mood and biases for depression-relevant words in 2 task blocks. Therefore, RTs indicated that the initial orienting response away from depression-relevant words was followed in time by a bias toward depression-relevant words. Regarding positive words, EMs showed a negative relation between dysphoria and the bias for positive words. Later in time, there was no relation between naturally-occurring dysphoria and a bias for positive words, as shown by RTs. For induced dysphoria, EMs showed that the depressed MIP-group oriented away from depression-relevant words in comparison to the neutral MIP-group. In addition, EMs showed that for positive words this relation reversed as the depressed MIP-group oriented their attention toward positive words in comparison with the neutral MIP-group. RTs showed a similar group difference for both word types. Therefore, these results indicate that the initial orienting response probably was maintained until probe onset, at least in part of the task blocks.

\section{Conclusions.}

General orienting processes and the time course of attentional bias for emotional stimuli were studied in naturally-occurring and induced dysphoria. General orienting results showed that all participants were able to orient their attention, but an induced negative mood led to a relative inability to disengage attention. ERP results only partly replicated previous studies with lateralized components at posterior sites. For the depressed MIP-group in comparison with the neutral MIP-group, the LDAP was smaller and there was a larger lateralized decrease in the La1-band at posterior sites. It was hypothesized that these group differences for lateralized activity might be related to the group difference in RT costs. Attentional bias results showed that biases varied over time in two important ways: (1) biases after word onset sometimes differed from biases after probe onset, and (2) bias effects on RT were unstable. Both types of variance over time may contribute to heterogeneous results of attentional bias in dysphoria and depression shown between studies. The results showed that naturally-occurring dysphoria was related to a bias away from depression-relevant words, followed by a bias toward these words or no bias, depending on task block. Naturally-occurring dysphoria was related to a similar bias away from positive words after word onset, but no bias was shown in RT results. The different dependent measures all indicated that induced 
oc chapter 6

dysphoria was related to a bias toward positive and away from depression-relevant words. This bias may function as a way to regulate emotion. 


\section{General Discussion}


In the first chapter of this thesis a theoretical overview was given of the main topics, and the goals were presented. These goals were: (1) To derive sensitive indices of orienting from the EEG to measure the time course of attention; (2) To determine the functional significance of these indices of orienting; (3) To examine processes of attention in situations where it is not directed by a cue, (4) To examine the role of sequence effects in a spatial cueing paradigm; (5) To examine the relation between general orienting processes and negative mood; (6) To examine the role of attentional bias in bipolar disorder and dysphoria; (7) To examine the time course of attentional bias in induced and naturally-occurring dysphoria. Five studies were conducted to address these issues. In chapters 2, 3, and 4 the focus was on EEG indices and response time measurement of spatial orienting. In chapter 5 and 6 attentional bias in bipolar disorder and dysphoria were examined. There were several main findings that are discussed here and will be related to each other by addressing each of the main issues. The chapter closes with some suggestions for further research.

\section{Indices of orienting in the EEG}

One of the main aims of the first study (chapter 2) was to derive sensitive indices of orienting from the EEG. Chapter 3 and 6 aimed to replicate the results from chapter 2 . In addition the functional significance of the indices was further examined in chapter 3 , and in chapter 6 the indices were used to examine the time course of processes of orienting in relation to negative mood.

In chapter 2, an endogenous spatial cueing paradigm was used and both RTs and the EEG were analyzed. In the paradigm, directional arrow cues were presented that pointed to the left or to the right and neutral cues that consisted of a double arrow. The task was a detection task and participants were motivated to direct their attention by giving the cues high predictive validity concerning the target location. Neutral cues had no predictive validity. First, averaged responses in the EEG for cued trials were examined to derive the indices of orienting. We reasoned that with valid indices, we should be able to determine the direction of attention in every single trial in which attention had been cued to the left or to the right, based on the single-trial EEG response after cue-onset. Response times were used for verification by comparing RT validity effects based on the cued direction of attention with RT validity effects based on the direction of attention based on the EEG response. As presented in Table 1 (an addendum to Table 1 presented in the introduction), averaged results showed a sequence of lateralized ERP components that mainly replicated the results of previous studies. The sequence consisted of a lateralized negativity between 250 and 500 ms contralateral to the direction of attention, reflecting an "early directional attention negativity" (EDAN) at posterior sites and an "anterior directing attention negativity" (ADAN) at anterior and central sites. At posterior sites this negativity was followed by a contralateral positivity between 500 and $1200 \mathrm{~ms}$, reflecting the late directing attention positivity (LDAP). In addition, there was a posterior contralateral positivity between 150 and 250 ms (not included in Table 1). Results of lateralized changes in EEG frequency bands extended previous results as changes were shown in the lower alpha1 (La1) band (6-8 Hz), but not in the classic alpha band $(8-12 \mathrm{~Hz}$ ), or in higher frequency bands. Importantly, single-trial ERP lateralizations were successfully used as predictor variables to classify 
the direction of attention in single trials with directional arrow cues in a reliable way. Predictions were successful with ERP responses, but not with changes in the La1-band. This showed that the lateralized ERP response, consisting of the components mentioned before, may serve as a valid index of orienting.

\section{Lateralized ERP components}

The conclusion in chapter 2 that lateralized ERP components were valid indices of orienting led to the question if these components could be specified more precisely in terms of functional significance. A first attempt was done at the end of chapter 2 , by examining the correlation of between-subjects variation in the different (averaged) components and the RT validity effect. A correlation would confirm that components were related to attentional orienting and not, for example, to cue asymmetries. Similarly, Driver and colleagues showed a correlation between the size of the ADAN and modulatory effects of attention on the P1 amplitude after target onset (Driver, Eimer, Macaluso, \& van Velzen, 2004). Our results showed a correlation only between the EDAN and the validity effect. This result appeared in contrast with the interpretation of this component proposed by Van Velzen \& Eimer (2003) who suggested that the EDAN was an N2pc component (Luck \& Hillyard, 1994; Eimer, 1996), reflecting the selection of lateralized cue parts. As mentioned in the introduction of this thesis, the cues used in their experiment required selection of a lateralized part and may at the same time have been incomparable to standard arrow cues and ideal in evoking an N2pc. Therefore, in chapter 3 the main aim was to further examine the functional significance of the lateralized ERP components. More specifically, we aimed to discriminate components related to sensory (lateralized) aspects of the cue and components related to the process of orienting, and therefore sensitive to the cued direction of attention. This was done by manipulating cue shape in two spatial cueing tasks. In one of the tasks, the physical properties of arrow cues were balanced across attention-left and attention-right instructions. This resulted in a congruent mapping $(<<$ for left, $>>$ for right) and an incongruent mapping ( $<<$ for right, $>>$ for left). Nonlateralized line cues of which the length determined the direction of attention were used in the other task. This resulted in an arbitrary mapping of line length and cue direction. Results are presented in Table 1.

Regarding components related to sensory aspects of the cues, results of chapter 3 showed an early posterior positivity between 150 and $250 \mathrm{~ms}$, also present in chapter two, that was related to the physical direction of arrow cues. Regarding components related to the direction of attention there were three important findings. First, the ADAN and LDAP were related to the direction of attention. This is in line with studies on crossmodal attention that have argued that the ADAN and LDAP might reflect activity in a supramodal mechanism in control of spatial attention (e.g., Eimer \& van Velzen, 2002; Eimer, van Velzen, \& Driver, 2002). Second, our results replicated previous studies in showing that modulatory effects of attention on sensory components after target onset depended on task demands and relevancy of the uncued position (e.g. Eimer, 1994; Mangun \& Hillyard, 1991). More important, these results were extended as it was shown that cue-related components similarly depended on task demands and relevancy of the uncued position. Therefore, in line with the correlation between ADAN and the P1 amplitude shown by Driver and colleagues (Driver et al., 2004), cue-related 
components and enhanced sensory processing after the target appear to be related to each other. Both were stronger when the task asked for more engagement of attention and the uncued position could be ignored. Third, there was a latency shift of the ADAN. It was elicited later in time in the incongruent mapping where the instructed meaning of arrow cues was reversed, and when an arbitrary mapping of line cues was used. In general, conditions that trigger a latency shift or modulate the amplitude of a component are of great value when studying the functional significance of ERP components. Here, in conditions where a delay was shown, the interpretation of the cues was more complex than the relatively automatic interpretation of arrow cues (Eimer, 1997; Tipples, 2002). Comparable results come from a study that showed a latency shift of the ADAN when auditory cues were used and participants were required to discriminate both the instrument and the pitch of the sound to determine cue meaning (Eimer \& van Velzen, 2002). The ADAN thus appears to be delayed whenever the demands of a cueing procedure are more complex.

In chapter 3 a third process was necessary, in addition to processing sensory aspects of the cue and the actual shift of attention, to explain the functional significance of the EDAN. That is, the EDAN was only elicited in conditions where arrow cues were used, and never with nonlateralized cues. In addition there was no evidence that the EDAN was related purely to processing the sensory aspects of arrow cues. The EDAN therefore appears necessary for orienting only when arrow cues are used. This is in line with the idea proposed by Van Velzen and Eimer (2003) that the EDAN is related to selection of lateralized parts. As argued before, one may doubt if the arrows that were used in our experiments required selection of lateralized parts. However, many of our participants, when participating in the incongruent arrow condition, mentioned that they paid attention to the open end of the arrows to derive the direction of the cue. If these subjects are representative, our results may be taken to suggest that the EDAN might be related to selection of a lateralized part (van Velzen \& Eimer, 2003) and in line with Harter and colleagues (1989), is related to the encoding of directional information. In the light of this encoding function, the correlation between EDAN and the RT validity effect in chapter 2 at first may be regarded as unexpected. However, the process of translating the symbol in a cued direction obviously is a necessary stage, early in the process of shifting attention, that may interact with stages of the shifting process that follow on cue interpretation. Larger amplitudes may represent stronger cue utilization and it is perfectly imaginable how this might influence costs and benefits of attention. For now, the positive correlation between EDAN size and the validity effect on RT may best be interpreted as follows: devoting more processing resources to cue interpretation or perhaps cue utilization has a beneficial effect on the validity effect.

In sum, regarding the functional significance of lateralized ERP components the results of chapter 2 and 3 have shown that processing the physical cue is reflected by an early posterior positivity and is followed in time by the EDAN that appears to reflect the translation of arrow cues, and perhaps also other kinds of lateralized cue symbols, into directional meaning. The ADAN may reflect control processes of shifts of attention that are delayed when deriving cue meaning is more complex. The LDAP has been assumed to reflect modulation of the cortical excitability in those regions that process the upcoming visual information (Harter et al., 1989). However, inconsistent with this interpretation, the LDAP decreased before target onset (Hopf \& Mangun, 2000; Van 
Velzen, Forster, \& Eimer, 2002) and was absent in a number of studies. In the present thesis two interesting results regarding the LDAP were derived from chapter 3 and chapter 6 . In chapter 6, lateralized ERPs were measured in a dot probe task that had a spatial cueing paradigm incorporated in it. Both findings for the LDAP concern the arrow stimuli. Responses to the word stimuli are discussed below. First, an early start of the LDAP, between 300 and $500 \mathrm{~ms}$, was shown in chapter 3 and in chapter 6 . Based on the results of chapter 3, a temporal and spatial overlap for the EDAN and the LDAP was hypothesized. It was reasoned that the LDAP may always have an early start, around 300 ms after cue-onset. However, as the amplitudes of the EDAN and LDAP are opposite, only the stronger one will be observed in the ERP, or they might cancel out against each other in case they are equally strong. At this point, this explanation of the data is still speculative and other techniques (e.g. source localization of both components) are necessary to confirm the overlap. The second interesting result regarding the LDAP comes from chapter 6 . The amplitude of the LDAP was smaller in one of the participating groups that showed larger attentional costs. This suggests a relation between the LDAP and disengagement processes of attention. However, as noted already in chapter 6 , in terms of cortical excitability the LDAP would have been expected to be larger when costs are larger. In addition, the modulation of the LDAP only was present in part of the group that showed larger costs.

These results on the functional significance of the lateralized components shed new light on the result of the classification of neutrally-cued $(<>)$ trials in chapter two. There, based on their success in classifying arrow-cued trials, the early posterior positivity, the posterior EDAN and LDAP, and the ADAN were all included as predictor variables to classify neutrally cued trials as 'attention-left' or 'attention-right' trials. In chapter three, however, it appeared that the first two of these components were most likely related to the physical appearance of the arrow-cue, not to the direction of attention, because they were absent in the conditions with vertical line-cues. Since the subsequent classification concerned the neutrally-cued $(<>)$ trials, these components might not add anything to successful classification. If so, they perhaps had even been better left out as predictor variables. This shows the importance of progression and new insights in the interpretation of ERP components, as well as the necessity of future studies to focus on deriving the functional interpretation of these components.

In chapter 6 , in a dot probe task, orienting processes were examined in response to laterally presented emotional words to demonstrate the time course of attentional bias. The results showed no significant lateralized ERP components. RT results strongly depended on task blocks and when blocks were pooled no attentional bias effects were observed. In this sense, the ERP effects were in line with the RT effects. As discussed in detail below (paragraph 7.5.2), RT bias results for individual task blocks showed some interesting results. Unfortunately, pooling task-blocks was inevitable for the ERP analyses to obtain good signal-noise ratio: if it had been possible to derive reliable ERPs from individual task-blocks, these could have been compared to RT results. Therefore, it is too early to draw the conclusion that lateralized ERP effects are unsuitable to measure processes of orienting in response to peripherally presented word stimuli. In relation to this, in chapter 3 we demonstrated that lateralized ERP effects were larger in a discrimination task than in a detection task. Thus, changing the dot probe task into a discrimination task by asking participants to classify the dot may enhance ERP effects. 
Finally, it should be noted that several nonlateralized ERP components have also been demonstrated to be related to processes of orienting (Slagter, Kok, Mol, \& Kenemans, 2005; Slagter, Kok, Mol, Talsma, \& Kenemans, 2005; Talsma, Slagter, Nieuwenhuis, Hage, \& Kok, 2005). To study this nonlateralized activity, left and right directing attention conditions were averaged and compared to a neutral reference condition. Our search for indices of orienting only concerned lateralized ERP components, for two reasons. First, nonlateralized activity is nonspecific regarding the direction of attention, so it will not tell if attention was moved toward or away from emotional content. Secondly, finding a valid neutral reference condition may not be easy. For example, in the dot-probe task, neutral-neutral word pairs would not be a valid neutral reference because emotional material is completely lacking there.

\section{Lateralized changes in EEG frequency bands}

The results from chapter 2 showed a lateralized decrease in the lower alpha1 (La1) band (6-8 Hz) at posterior sites between 400 and $1200 \mathrm{~ms}$ after cue-onset, contralateral to the direction of attention. There were no lateralized changes in the classic alpha band $(8-12 \mathrm{~Hz})$, or in higher frequency bands. In addition, predictions of the direction of attention based on single trial EEG responses were not successful when changes in the La1 band served as predictor variables. In previous studies that analyzed changes in EEG frequency bands, lateralized changes in the alpha band and gamma band were shown in a cueing paradigm after cue onset (Gruber, Muiller, Keil, \& Elbert, 1999; Worden et al., 2000). Importantly, in both studies the paradigm that was used required participants to discriminate the target and ignore the uncued target position. In chapter 3 it was shown that there was a relation between ERP activity after cue onset and task instruction. If participants were to completely ignore events at the uncued position and had to discriminate the target, this had an enhancing effect on lateralized ERP components. Therefore, lateralized changes in the EEG frequency bands may similarly depend on task demands and the relevance of the uncued position. New experiments or frequency band analyses of the data presented in chapter 3 would therefore be of interest.

In chapter 6, lateralized changes in the La1-band after arrow stimuli at posterior sites were examined in order to find out if averaged results would replicate the findings of chapter 2. The task that was used in chapter 6 was a combination of a spatial cueing paradigm and a dot probe task. The spatial cueing paradigm was a detection task that was highly similar to the task that was used in chapter 2 . There was a significant validity effect and there were benefits in all participants. Lateralized changes in the La1-band were only minimal however. Although there was an effect again that started at $400 \mathrm{~ms}$ after cue onset, it only lasted until $600 \mathrm{~ms}$ after cue-onset. In addition the expected decrease in La1-activity contralateral to the cued position was present only in part of the participants whereas an increase was shown in one of the groups. As for ERPs, lateralized changes in the La1-band were also examined in response to peripherally presented word stimuli in the dot probe paradigm. There only were two very shortlasting effects, one for depression-relevant words (600-800 ms) and one for positive words (400-600 ms). Both effects were limited to one electrode-pair. As these effects for both arrow stimuli and word stimuli were small in comparison to the effect that was shown in chapter 2 , we refrained from drawing strong conclusions about the functional significance of these changes. In all, the results from chapter 6 confirm the conclusion 
derived from the classification analysis in chapter 2 that lateralized changes in the La1band appear no robust indices of orienting. However, as already mentioned, similar to ERP components after cue-onset, lateralized changes in frequency bands might be enhanced when task instruction is changed. Above all, these results demonstrate the need to focus on the optimal conditions to elicit lateralized changes in frequency bands first before these measures are used in an applied context. Furthermore, it should be examined if different time windows after cue onset can be related to different sub-processes in the process of orienting in a way similar as this has been studied for lateralized ERP components.

\section{Attention in neutral trials}

In the final step of the analyses in chapter 2, lateralized ERP components were used to determine if attention was shifted in the neutral trials. This served to provide an answer to an existing discussion in the literature on what happens with attention when a non-directional cue is presented. The results showed no evidence that attention was shifted in neutral trials in a way similar to shifts of attention in cued trials. As noted in paragraph 7.1, statistical power of the analysis may not have been optimal to discriminate shifts of attention in neutral trials. However, EEG-analyses of nonspatial attention showed within-subject variations in preparation after a neutral cue, reflected by modulation of the CNV component and nonlateralized alpha band activity. This variation in the EEG was successfully detected and explained part of the variance in RTs. It was taken as evidence that variations in the RTs of neutral trials are not merely due to meaningless noise but are indeed related to variations in attention.

An important new finding regarding neutral trials comes from the study presented in chapter 4 . In this study sequence effects were explored in a spatial cueing paradigm. It was shown in "current" neutral trials that the position that was endogenously cued in the previous trial was inhibited. This effect was independent of the exogenously cued target position in the previous trial which is striking, but in line with studies that showed that endogenous and exogenous effects of attention can be independent and additive (e.g., Berger, Henik, \& Rafal, 2005; Berlucchi, Chelazzi, \& Tassinari, 2000). The inhibitory effect was taken as evidence that endogenous shifts of attention result in long-term inhibitory processes. Many studies have failed to show effects of IOR in an endogenous cueing paradigm (for a review, see Klein, 2000), unless the endogenous cue triggered the oculomotor system (Rafal, Calabresi, Brennan, \& Sciolto, 1989). Importantly, these studies all focused on IOR within trials and as noted by Taylor and Klein (2000) any inhibitory effects may consequently have been overpowered by facilitatory effects of voluntary orienting. Similarly, the effect was specific for neutral trials in the study presented in chapter 4 , as there was no evidence for long-term inhibitory effects in cued trials. However, in an unpublished pilot study from our lab the inhibitory effect of the previous cue was shown in neutral trials and cued trials. In the cued trials the validity effect of the current trial and the inhibitory effect of the previous trial were additive. Notably, the validity effect in the "current" trial was smaller in the pilot experiment than in chapter 4 which suggests that the overpowering facilitatory effect may have been smaller. 
In sum, the results from chapter 2 and 4 demonstrate two possible sources of RT variance in neutral trials. The results in chapter 2 showed that part of the RT variance was due to variations in preparation (non-spatial attention) that were shown in the EEG. The effect of long-term IOR that was shown in chapter 4 shows that spatial orienting processes in the previous trial represent another source of RT variance in neutral trials. One may wonder why these effects of IOR that were measured by RTs were not picked up by the classification method that was used in chapter 2 . Probably, this is due to the predictor variables that were used in the classification method as these consisted of lateralized changes after cue-onset with respect to the baseline period. From this it follows that any effect that was already present before cue onset (and continued after cue onset) would not result in lateralized changes after cue onset.

\section{Sequence effects in a spatial cueing paradigm}

As mentioned previously, the goal of chapter 4 was to explore sequence effects in a spatial cueing paradigm. Inhibitory results for neutral trials have already been described. In addition there were two important findings. First, results of previous studies were replicated as it was shown that after a catch trial, responses were slower (Alegria, 1978; Correa, Lupiáñez, Milliken, \& Tudela, 2004; Los, 2004). Importantly, these results extended previous results by showing that costs and benefits remained intact. This dissociation supported the discrimination by Posner and Boies (1971; Posner \& Petersen, 1990) that was mentioned in the introduction, of processes of attention that heighten general alertness and processes that selectively prepare a subject, for example for a location. Second, strong strategic effects were shown in cued trials as costs and benefits were larger after a valid trial than after an invalid trial. This suggests that participants strategically adapted their utilization of the cues. In chapter 2, as mentioned previously, a correlation between the amplitude of the EDAN and validity results was shown. It was speculated that this might be related to cue utilization. It would therefore be very interesting to measure EEG in a study on sequence effects similar to the one presented in chapter 4 . A reduction of the amplitude of the EDAN after an invalid trial in comparison to after a valid trial would support our hypothesis about cue utilization.

Above all, this study not only shows that analyses of sequence effects can enhance our insights in processes that influence task performance but also that these effects may be important contributors to RT variance. Therefore, instead of leaving trial-order a random factor, controlling trial sequences as it was done in chapter 4 , may be recommendable in general. However, one of the obvious disadvantages is the increasing number of trials when the number of conditions increases. This may for example facilitate the use of (unwanted) strategies, or in some cases may not be possible because experiments will take too long as in the case of testing clinical groups.

\section{General orienting and negative mood}

Interestingly, a similar interplay of mood and general orienting processes was shown in chapter 5 and 6 . In both studies negative mood was related to a relative inability to disengage attention, when directed by arrow cues. Notably, in chapter 5 it concerned naturally occurring negative mood in a group of patients with bipolar disorder, and in chapter 6 the relation was shown for induced dysphoria. As BDI scores were consider- 
ably higher in the patient group, this suggests that the relation is present for naturallyoccurring negative mood only in more extreme cases of negative mood. This result extends the outcome of a study by Compton (2000) mentioned in the introduction. Furthermore, it is in line with the suggestion by Bradley et al. (1997) that disengagement problems may be the underlying mechanism of attentional biases for emotional stimuli in depression and dysphoria. Some evidence for this has already been shown (Ellenbogen, Schwartzman, Stewart, \& Walker, 2002; Koster et al., 2005). Importantly, however, our data showed that disengagement problems are not specific for negative words. Instead negative mood appears to be related to a core deficit to disengage attention. For future studies it will be important to further examine how disengagement problems may relate to attentional bias and emotional processing. As it has been shown that parietal areas are important for disengagement (e.g., Posner \& Petersen, 1990), imaging studies may be of interest.

\section{Attentional bias}

In chapter 5 , the role of attentional bias in bipolar disorder was studied for the first time by using a dot probe paradigm. In chapter 6 , the time course of attentional bias was studied in induced and naturally-occurring dysphoria.

\section{Attentional bias in bipolar disorder}

The task in chapter 5 was a combination of a dot probe task and a spatial cueing paradigm. A group of bipolar patients participated and was divided into a euthymic group and a mildly depressed group. Different group comparisons allowed an examination of trait (euthymic patients versus healthy controls) and state characteristics (euthymic versus mildly depressed patients) regarding attentional bias. The results are presented in Table 2 (an addendum to Table 2 in the introduction). The results were surprising as they showed no evidence for a mood-congruent bias toward negative words in the mildly depressed group. Instead there was (1) a bias away from positive words that acted as a trait characteristic, equally present in both patient groups, and (2) a bias away from negative (depression-relevant) words that was state-dependent, as there was a strong negative correlation between negative mood and attentional bias within the patient group. It was hypothesized that biases in bipolar disorder might be related to the transition of mood states that is characteristic for bipolar disorder. A bias away from all emotional information in the mildly depressed state might predict a transition to a euthymic state, and a bias away from positive words and toward depression-relevant words might predict a transition from the euthymic state to one of the other mood states.

\section{Attentional bias in induced and naturally-occurring dysphoria}

The study in chapter 6 was an extension of the study by Bradley et al. (1997). The most important change in our study was the goal to measure the time course of attentional bias by using indices of orienting derived from the EEG and measurement of eye movements after word onset. The dot probe paradigm that was used was the one presented in chapter 5. However, in order to improve the signal-noise ratio for EEG measurement, the number of trials was doubled. A factorial design was used to study induced dysphoria and naturally occurring dysphoria in one study. Two groups of participants were 
tested that varied in mood from healthy to dysphoric. One of these groups underwent a neutral MIP and the other group underwent a depressed MIP. Reaction time results are shown in Table 2. There were a number of main findings.

First, doubling the number of trials had a surprising effect concerning the time course of attentional bias as there were large differences in biases between the different task blocks. Moreover, and importantly, there were no significant differences in attentional bias when the results of the four task blocks were averaged. This type of variance over time may not only contribute to the heterogeneous results of attentional bias in depression and dysphoria, but in addition puts into question the idea that attentional bias would serve as a stable trait characteristic. It also shows the importance of including the factor task-block in the statistical design when blocks have been presented repeatedly in a study. Two studies that aimed to examine the time course of attentional bias also showed differences between task blocks (Liu et al., 2006; Schmukle, 2005). Both studies concerned non-clinical anxiety. Perhaps these fluctuations between blocks are characteristic only for non-clinical anxiety and dysphoria. These results show the importance for future studies to systematically examine the time course of attentional bias across different task blocks.

In addition to the time course across task blocks, this study confirmed the importance of tapping attention at different points in time within trials. Eye movements in particular were useful in demonstrating the time course of attentional bias immediately after word onset. The combination of eye movements and response times thus allowed us to describe the time course of attentional bias within trials. Notably, the pattern of eye movement biases was stable across task blocks. It was shown that early in time, after word-onset, naturally-occurring dysphoria was related to a bias away from all emotional word stimuli. However, this initial orienting response in naturally-occurring dysphoria was followed in time by a bias toward depression-relevant words or no bias, as there never was a RT bias for positive words and no RT bias for depression-relevant words in some of the task blocks. For induced dysphoria, the depressed MIP-group in comparison to the neutral MIP-group showed an initial orienting response away from depression-relevant and toward positive words. This pattern was maintained after probe onset (as shown by a similar RT pattern) or again followed by no bias in some of the task blocks. This pattern opposing mood-congruency has been shown by others (Ellenbogen, Schwartzman, Stewart, \& Walker, 2002; Miranda, Gross, Persons, Hahn, 1998) and our results extend on these results by showing that the pattern already started early in time, after word onset. It may reflect an adaptive mechanism to regulate emotion. It was speculated that the early avoidance response away from all emotional stimuli in naturally-occurring dysphoria may similarly serve to regulate and attenuate emotional arousal in those scoring higher on dysphoria. However, in part of the task this regulatory mechanism eventually broke down, as naturally dysphorics were shown to orient toward depression-relevant words in some of the task-blocks. This failing regulatory mechanism may be one of the cognitive vulnerabilities that characterize dysphoria and perhaps eventually may lead to depression. All of this is highly speculative of course and there is a need for replication. Above all, the measurement of eye movements for the first time provided evidence for early attentional biases in dysphoria. Other studies have shown no evidence for biases in dysphoria or depression when brief, masked stimulus exposures were used and RT biases were measured. Therefore, as suggested 
in chapter 6, EMs may provide a more sensitive measure of attentional bias. Above all, these results show the importance of tapping attention at different points in time. The distinction between initial orienting and maintenance of attention may be important in contributing to the heterogeneous findings across studies (Mogg et al., 2000).

\section{Attentional bias in bipolar disorder versus attentional bias in dyspho- ria}

Although the same paradigm was used in chapter 5 and 6 , the task in chapter 5 only contained half of the trials and only RT biases were analyzed. ${ }^{1}$ A comparison of RT biases in both studies shows that whereas naturally occurring dysphoria was related to a mood-congruent bias toward depression-relevant words (although inconsistently) and no bias for positive words, a depressed state in bipolar disorder was related to a bias away from depression-relevant words. This seems to confirm our hypothesis that biases in bipolar disorder differ from biases in dysphoria and unipolar depression, and may be related to the transition of mood states characteristic for bipolar disorder. However, although RT biases were different, the bias in bipolar disorder was similar to the early bias in dysphoria demonstrated by eye movement measurement. A bias away from all emotional words in bipolar disorder was hypothesized to predict a transfer to a euthymic state. This is in line with the idea that the bias serves as a coping mechanism to regulate emotion. When the time course is considered, mildly depressed patients appear more successful in this process of regulation as it was shown in dysphorics that the bias sometimes turned into a mood-congruent bias after target onset. Perhaps this is due to the fact that all patients were on medication that served to regulate their mood states. Clearly, this is all speculative and asks for a number of new studies, among others to find out if bipolar patients also demonstrate this bias early in time, after word-onset. In addition replication of effects is necessary in bipolar disorder because this was the first time a dot-probe paradigm was used, and in dysphoria because this was the first study that measured eye movements to demonstrate the time course of attentional bias.

\section{Future directions}

A number of promising avenues and recommendations for future research follow from the work presented in this thesis. Firstly, the classification method that was described in chapter 2 shows that single trial ERP responses have more potential than is often suggested. The classification method might prove useful in the future, in experiments where the dependent variable consists of two categories that can be discriminated by an ERP response. Secondly, sequence effects may be responsible for a part of the (error) variance in every experiment. Presenting trials at random but restricting trial sequences might therefore be advisable in all cases to reduce error variance. Thirdly, it followed from the studies presented here that future studies should focus on deriving the functional interpretation of the lateralized ERP components and of the different time windows of lateralized changes in EEG frequency bands presented here. With a better understanding of the function of their generators, both will be of high value to study processes of attention in various applied experiments. Specification of the condi-

1 Although EEG was also measured in the clinical study presented in chapter 5, unfortunately these data were of bad quality, containing too much noise to be interpretable. 


\section{8 chapter 7}

tions and task demands that are required to obtain reliable effects furthermore seems necessary. Fourthly, future studies should focus again on the role of attentional bias in dysphoria, unipolar depression, and bipolar depression. For bipolar disorder, studying patients in different mood states may be helpful to understand the role of attentional bias in mood regulatory processes. In an ideal experiment, patients are tested repeatedly in a within-subjects design, in different mood states. Fifthly, studies on attentional bias should systematically examine the time course across task blocks, as inconsistency of effects across blocks may be one of the factors that causes heterogeneous results. Finally, studying the time course of attentional biases in dysphoria has shown to be of great value. New attentional bias studies that continuously monitor attention are necessary to replicate the results for dysphoria and find out if these results extend to unipolar and bipolar depression. 


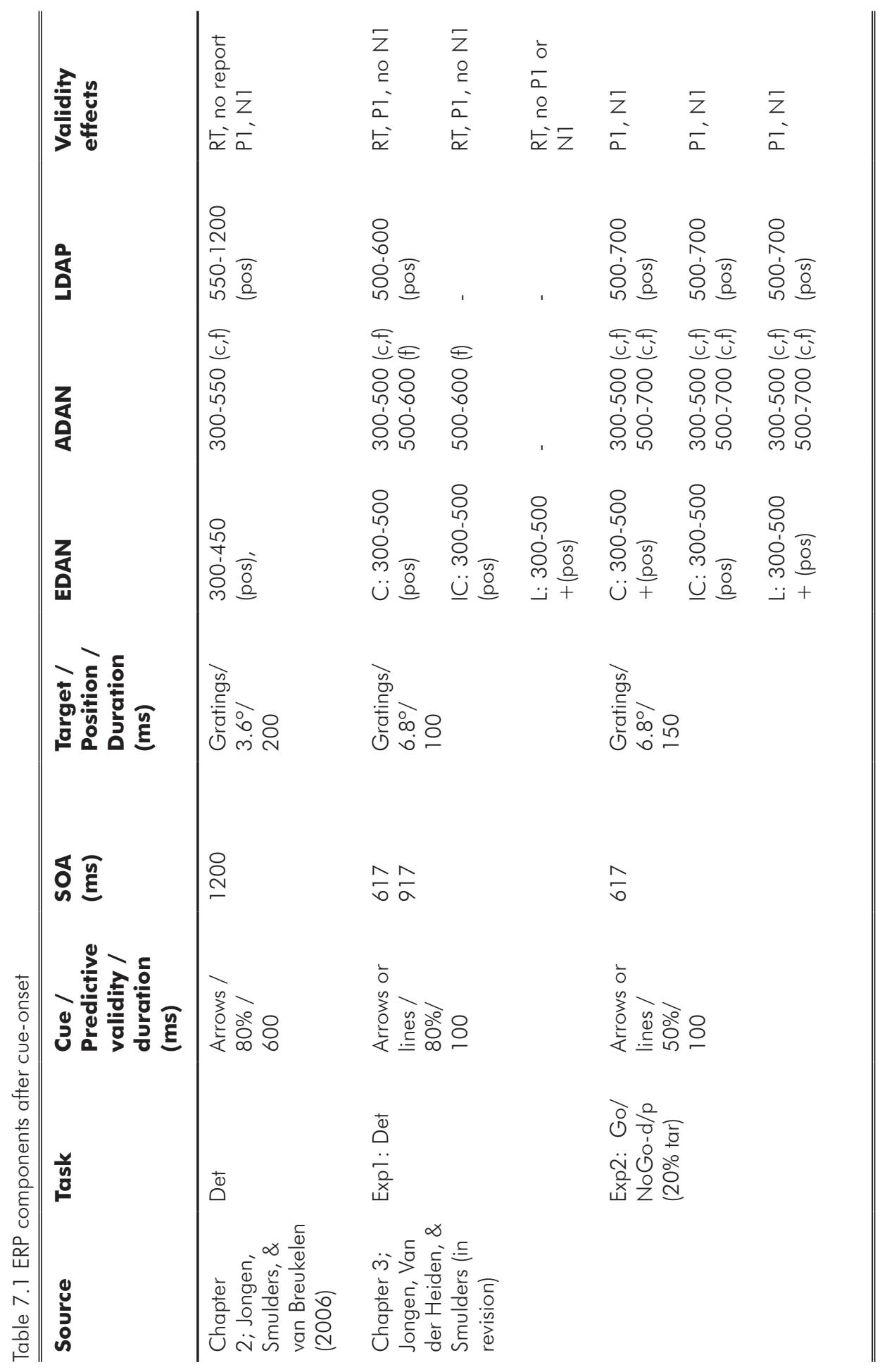




\section{8 chapter 7}

\section{Alphabetical list of abbreviations ( $p /$ column) in Table 7.1:}

Task

Det, detection task; detection of a target stimulus by pressing a response button as fast as possible

Exp, experiment

Go/NoGo-d/p, go/nogo discrimination/position task; responses only to target stimuli, only when presented at cued positions, one response button

tar, target stimuli

EDAN / ADAN / LDAP

ant, anterior

c, central

C, congruent arrow condition

f, frontal

IC, incongruent arrow condition

L, line condition

pos, posterior

Validity effect

i, invalid

n, neutral

RT, response times

$\mathbf{V}$, valid 

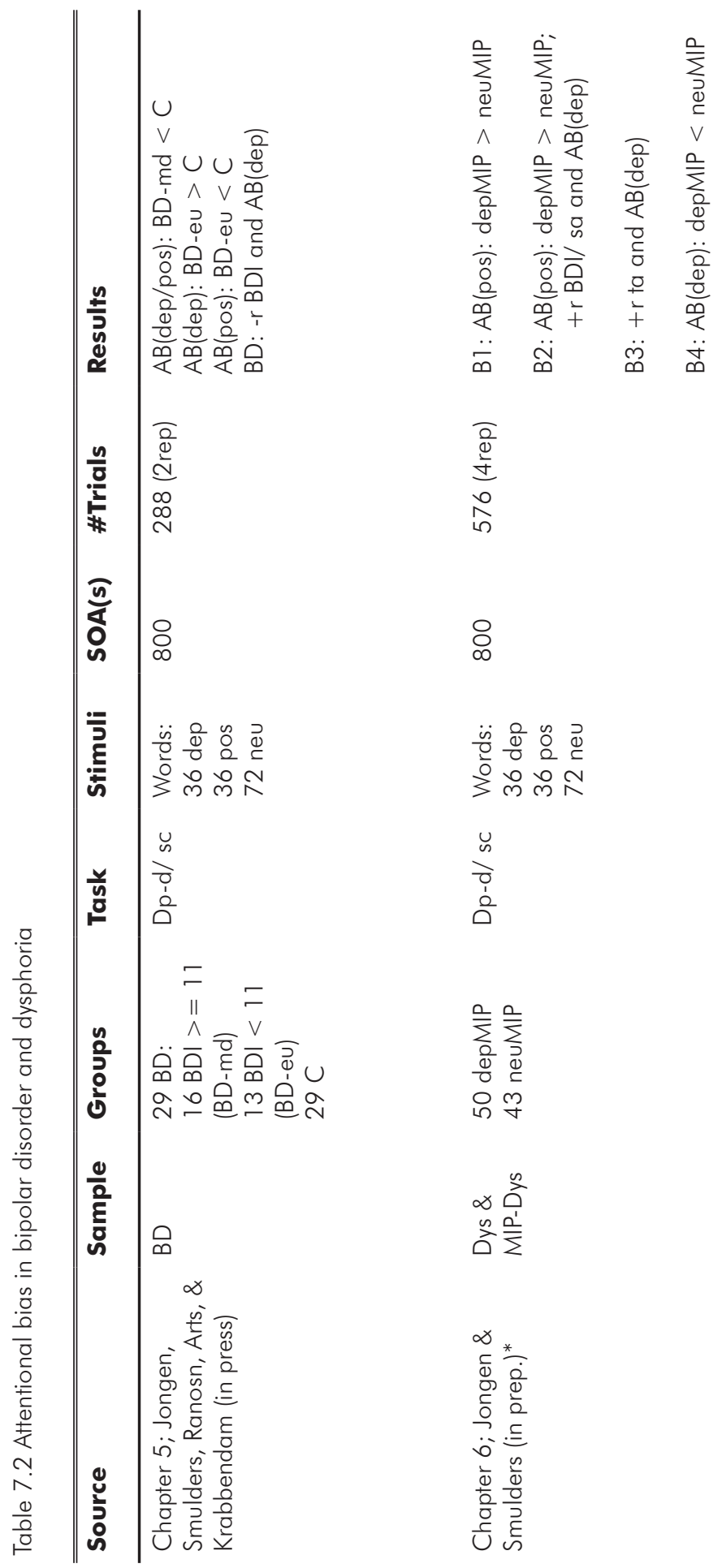

붕웡 웜 కి mల

$u$
ò
ेे
$\dot{0}$

$\sum_{\substack{0 \\ \frac{0}{0}}}^{\substack{0 \\ 0}}$

윰

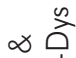

帘高

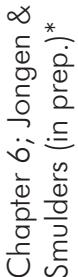

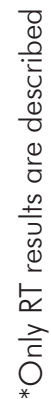




\title{
Alphabetical list of abbreviations ( $p$ / column) in Table 7.2:
}

\author{
Sample / Groups \\ BD, bipolar disorder \\ BD-eu, bipolar disorder, euthymic \\ BD-md, bipolar disorder, mildly depressed \\ BDI, Beck Depression Inventory \\ C, heathy controls \\ depMIP, depressed Mood Induction Procedure \\ Dys, dysphorics \\ MIP-dys, mood induced dysphoria \\ neuMIP, neutral Mood Induction Procedure
}

Task

Dp-d/ sc, combination of a dot probe detection task and spatial cueing paradigm

Stimuli

dep, depression-relevant stimuli

neu, neutral stimuli

pos, positive stimuli

\#Trials

rep, number of repetitions $\mathrm{p} / \mathrm{stimulus}$

Results

AB, attentional bias

B, Block

r, correlation

sa, state anxiety

ta, trait anxiety

-, negative / away from

+ , positive / toward 
Summary 
The central theme of this thesis is visuospatial selective attention. This cognitive mechanism helps us to prioritize relevant information and inhibit less important information in the visual domain. In addition to general processes of visuospatial attention (Chapter 2-6), the interplay of attention and negative mood was studied (Chapter 5, 6). Adjusted versions of a spatial cueing paradigm (e.g., Posner, 1980) were used in each of the empirical chapters in this thesis to study processes of spatial attention. To study the relation of attention and negative mood, an adjusted version of a dot-probe task was used. This task was derived from the spatial cueing paradigm. Attention can be directed overtly, by moving the eyes, or covertly, without co-occurring movements of the eyes. In all of our studies, we were interested in covert shifts of attention. Only in chapter 6 , both covert and overt shifts of attention were measured. In addition to response times, brain activity was recorded in part of the experiments by using the electroencephalogram (EEG) (Chapter 2, 3, 6). This is a non-invasive technique that allows mapping the time course of cognitive processes with millisecond precision. Here it served to measure the time course of spatial orienting. Two measures were derived from the EEG: (1) Event-Related Potentials (ERPs) (Chapter 2, 3, 6), and (2) Event-Related Changes in Oscillations (Chapter 2,6). Experiments were set up in such a manner that shifts of attention should result in processing differences between cerebral hemispheres. Therefore, the main focus was on lateralized ERPs and lateralized changes in oscillations, with a positive or negative voltage over the hemisphere contralateral to the direction of attention.

In Chapter 1, a theoretical introduction was given on the main topics: (visuospatial selective) attention, measures derived from the EEG (in general and in attention research), mood disorders, and the interplay of negative mood and attention in dysphoria, depression, and bipolar disorder. Furthermore, the goals of the thesis were presented. These were: (1) To derive sensitive indices of orienting from the EEG to measure the time course of attention; (2) To determine the functional significance of these indices of orienting; (3) To examine processes of attention in situations where it is not directed by a cue, (4) To examine the role of sequence effects in a spatial cueing paradigm; (5) To examine the relation between general orienting processes and negative mood; (6) To examine the role of attentional bias in bipolar disorder and dysphoria; (7) To examine the time course of attentional bias in induced and naturally-occurring dysphoria. Five studies were conducted to address these issues. In chapters 2, 3, and 4 the focus was on EEG indices and response time measurement of spatial orienting. In chapter 5 and 6 attentional bias in bipolar disorder and dysphoria were examined. For a general discussion of the results the reader is referred to chapter 7 .

In Chapter 2, in an endogenous spatial cueing paradigm, a directional arrow cue that pointed to the left or to the right was presented on every trial, or a neutral cue that consisted of a double arrow. Participants were instructed to indicate detection of a target stimulus that followed a cue by pressing a button as fast as possible. They were motivated to direct their attention on cue presentation because the cues had high predictive validity concerning the target location. Neutral cues had no predictive validity. The main goal of this study was to examine processes of spatial attention in neutral trials. This served to provide an answer to an existing discussion in the literature on what happens with attention when a non-directional cue is presented. For this goal, it was necessary to derive sensitive indices of orienting from the EEG. First, 
averaged responses in the EEG for (left and right) cued trials were examined to derive the indices of orienting. We reasoned that with valid indices, we should be able to determine the direction of attention in every single trial in which attention had been cued to the left or to the right, based on the single-trial EEG response after cue-onset. Response times were used for verification by comparing RT validity effects based on the truly cued direction of attention with RT effects based on the direction of attention that was "predicted" by the EEG response. Averaged results showed a sequence of lateralized ERP components that mainly replicated the results of previous studies (e.g. Eimer \& Van Velzen, 2002; Harter, Miller, Price, LaLonde, \& Keyes, 1989; Hopf \& Mangun, 2000; Nobre, Sebestyen, \& Miniussi, 2000). The sequence consisted of a posterior contralateral positivity between 150 and $250 \mathrm{~ms}$ followed by a lateralized negativity between 250 and 500 ms contralateral to the direction of attention, reflecting an "early directional attention negativity" (EDAN) at posterior sites and an "anterior directing attention negativity" (ADAN) at anterior and central sites. At posterior sites this negativity was followed by a contralateral positivity between 500 and $1200 \mathrm{~ms}$, reflecting the late directing attention positivity (LDAP). Results of lateralized changes in EEG frequency bands extended previous results as changes were shown in the lower alpha1 (La1) band (6-8 Hz), but not in the classic alpha band $(8-12 \mathrm{~Hz}$; Worden, Foxe, Wang, \& Simpson, 2000), or in higher frequency bands (Gruber, Muiller, Keil, \& Elbert, 1999). In the second step of the analyses, single-trial ERP lateralizations were successfully used as predictor variables to classify the direction of attention in single trials with directional arrow cues. Predictions were successful with ERP responses, but not with changes in the La1-band. This showed that the lateralized ERP response consisting of the components mentioned before may serve as a valid index of orienting. In addition it shows that single-trial ERP responses have more potential than is often suggested. Therefore, the classification method might prove useful in the future, in experiments where the dependent variable consists of two categories that can be discriminated by an ERP response. In the final step of the analyses, lateralized ERP components were used to determine if attention was shifted in the neutral trials. Again single-trial ERP lateralizations were used as predictor variables to classify every neutral trial as "attention to the left" or "attention to the right". An analysis of response times that was used to evaluate the classification showed no evidence for shifts of attention in neutral trials. However, an analysis of nonlateralized EEG activity (alpha (8-14 Hz) activity and the CNV component) in the cue-target interval showed that variations in response times of neutral trials were due to variations in nonspatial attention (general alertness) and therefore not merely due to meaningless noise.

The lateralized ERP components that were shown in Chapter 2 after cue-onset are thought to reflect different stages in the control of attention. In Chapter 3, we aimed to discriminate components related to sensory aspects of the cue and components related to shifts of attention. This was done by manipulating cue shape and task instruction in two endogenous spatial cueing tasks. In one of the tasks, the physical properties of arrow cues were balanced across attention-left and attention-right instructions. This resulted in a congruent mapping ( $<<$ for left, $>>$ for right) and an incongruent mapping ( $<<$ for right, $>>$ for left). A comparison of these mappings would show what components were related to the direction of attention and what components were related to the physical cue shape. Nonlateralized line cues of which the length determined the 
direction of attention (e.g. "|" for left, "|" for right) were used in the other task. This resulted in an arbitrary mapping of line length and cue direction. With nonlateralized cues it was possible to exclude the possibility that lateralized activity after cue-onset could have been elicited by shape characteristics of the cue stimulus. Both tasks were used in two experiments that differed in task instruction. In the first experiment, both validly and invalidly cued stimuli required a response, whereas in the second experiment responses were required only to infrequent target stimuli on valid trials. Our results replicated previous studies (e.g. Eimer, 1994; Mangun \& Hillyard, 1991) in showing that modulatory effects of attention on early sensory components after onset of the peripheral stimuli depended on task demands and relevance of the uncued position, as they were stronger in the second experiment than in the first. Regarding components after cue-onset, an early lateralized posterior positivity between 150 and $250 \mathrm{~ms}$, also present in Chapter 2, was related to the physical shape of arrow cues. The ADAN and LDAP were shown to be independent of the physical shape of cues and were also elicited when nonlateralized line cues were used to direct attention. This shows that these components are related to the direction of attention. However, both components were smaller or even absent when modulation of early sensory effects after onset of the peripheral stimulus was diminished or absent (in experiment 1), suggesting a relation with enhanced sensory processing after onset of the peripheral stimulus. Furthermore, in line with one other study (Eimer \& Van Velzen, 2002) the ADAN was delayed whenever the demands of the cueing procedure were more complex, in the incongruent mapping and when the arbitrary mapping was used. The data also suggested temporal and spatial overlap of the LDAP and the EDAN, which might explain why the latter was elicited only in some task conditions. A third process was necessary, in addition to processing sensory aspects of the cue and the actual shift of attention, to explain the functional significance of the EDAN. That is, the EDAN was only elicited in conditions where arrow cues were used, and never with nonlateralized cues. In addition there was no evidence that the EDAN was related purely to the physical shape of arrow cues. It therefore was concluded that the EDAN appears necessary for orienting only when arrow cues are used and might reflect the encoding of directional information of arrows and perhaps also other types of lateralized cue stimuli.

In Chapter 4, sequence effects were examined in an endogenous spatial cueing paradigm. The paradigm was similar to the one that was used in Chapter 2. However, to control the number of trial sequences, all possible sequences were balanced, leading to a large increase in the number of trials. The effect of the previous cue direction, the previous trial type (valid, invalid, neutral, catch), and target alternation effects were studied. The results showed strong strategic effects in cued trials as RT costs and benefits of attention were larger after a valid trial than after an invalid trial. This demonstrates that participants adapted their utilization of the cues: after an invalid cue, they oriented less strongly on the next trial. Second, although responses were slower after a catch trial (Alegria, 1978; Correa, Lupiáñez, Milliken, \& Tudela, 2004; Los, 2004) costs and benefits of attention remained intact, supporting the independence of attentional mechanisms of orienting and alertness (Posner \& Boies, 1971; Posner \& Petersen, 1990). Finally, in neutral trials orienting was influenced by the direction of the cue in the previous trial. More specifically, the position that was cued in the previous trials was inhibited. The inhibitory effect was taken as evidence that endogenous shifts of attention 
result in long-term inhibitory processes that are independent of exogenous effects and validity of the previous trial. This independence is remarkable because the attentional response to the previous target is closer in time than the response to the previous cue. However, it is in line with studies that showed that endogenous and exogenous effects of attention can be independent and additive (e.g., Berger, Henik, \& Rafal, 2005; Berlucchi, Chelazzi, \& Tassinari, 2000).

In Chapter 5, attentional biases for emotional word stimuli and general orienting processes were examined in bipolar disorder. A modified dot-probe task was used with a spatial cueing paradigm incorporated in it. Similar to a standard dot-probe task, a word pair was presented on every trial, with one word left and one word right from fixation. Emotional-neutral word pairs consisted of a depression-related or a positive word paired with a neutral word, and neutral-neutral word pairs were added as fillermaterial. The spatial cueing paradigm was similar to the one that was used in Chapter 2. A word pair and an arrow cue were presented simultaneously on every trial. The different types of word pairs were combined with predictive and non-predictive arrows in a way that allowed independent measurement of attentional bias for emotional words and general orienting processes within the same task. Bipolar patients in a euthymic state (i.e. remission), bipolar patients in a mildly depressed state, and nonpsychiatric controls participated. Results from the spatial cueing paradigm showed that all groups were able to orient their attention in response to arrow cues, as demonstrated by RT benefits. However, within the patient group, a depressed mood was positively related to RT costs, reflecting the relative inability to disengage attention. Attentional bias results showed no evidence for a mood-congruent bias towards negative words in the mildly depressed group. Instead, bipolar patients in a mildly depressed state, in comparison with controls, directed their attention away from depression-related words and positive words. The bias away from positive words was equally present in both patient groups and part of a trait effect, demonstrated by the comparison of patients in a euthymic state and controls. The bias away from depression-related words was mood state-dependent and within the patient group as a whole correlated negatively with measures of depressed mood. It was hypothesized that these effects of attentional bias are related to the transition of mood states, characteristic for bipolar disorder. Specifically, a bias away from all emotional information in the mildly depressed state might predict a transition to a euthymic state, and a bias away from positive words and toward depression-relevant words might predict a transition from the euthymic state to one of the other mood states.

In Chapter 6, attentional biases for emotional word stimuli and general orienting processes were examined in induced and naturally-occurring dysphoria. The time course of these processes was examined by measuring eye movements (EMs) and EEG after word-onset and arrow-onset. Similar to Chapter 2, lateralized ERPs and lateralized changes in the La1-band were extracted from the EEG. The paradigm that was used was similar to the one used in Chapter 5. However, to improve the signal-noise ratio for EEG measurement, the number of trials was doubled. Two groups of participants were tested that varied in mood from healthy to dysphoric. One of these groups underwent a neutral mood induction procedure (MIP) and the other group underwent a depressed MIP. Results from the spatial cueing paradigm showed that all participants were able to orient their attention in response to arrow cues, as demonstrated by RT benefits. 
However, RT costs, reflecting the relative inability to disengage attention, were higher in the depressed MIP group than in the neutral MIP group. This is comparable with the relation between naturally-occurring negative mood and RT costs that was shown in Chapter 5. It suggests that negative mood is related to a core problem in disengaging attention. ERP results and lateralized changes in the La1-band partly replicated the results of Chapter 2. Attentional bias results showed that biases varied over time, both within trials and between the different task blocks. When all blocks were averaged there were no significant differences in attentional bias. Therefore, these results show the importance of tapping bias at different moments in time within trials and of including the factor "task-block" in the statistical design if blocks have been presented repeatedly. These types of variance over time may contribute to the heterogeneous results of attentional bias in depression and dysphoria. Eye movements in particular were useful in demonstrating the time course of bias immediately after word onset. The combination of EMs and RTs thus allowed us to describe the time course within trials. EM-analyses showed that early in time, after word-onset, naturally-occurring dysphoria was related to a bias away from all emotional word stimuli. However, RT results indicated that this initial orienting response was followed in time by a bias toward depression-relevant words or no bias, as there never was a RT bias for positive words and a RT bias for depression-relevant words in only some of the task blocks. For induced dysphoria, EM analyses indicated that the depressed MIP-group in comparison to the neutral MIP-group showed an initial orienting response away from depression-relevant and toward positive words. RT results showed that this pattern was maintained after probe onset or again followed by no bias in part of the task blocks. In sum, these results again showed minimal or no evidence for a mood-congruent bias (similar to Chapter 5) in naturally-occurring and induced dysphoria, respectively. The pattern of biases shown in induced dysphoria has been shown by others (Ellenbogen, Schwartzman, Stewart, \& Walker, 2002; Miranda, Gross, Persons, Hahn, 1998) and our results extend these results by showing that the pattern already started early in time, after word onset. It may reflect an adaptive mechanism to regulate emotion. It was speculated that the early avoidance response away from all emotional stimuli in naturally-occurring dysphoria may similarly serve to regulate and attenuate emotional arousal in those scoring higher on dysphoria. However, in part of the task this regulatory mechanism eventually broke down, as naturally dysphorics were shown to orient toward depression-relevant words in some of the task-blocks. This failing regulatory mechanism may be one of the cognitive vulnerabilities that characterize dysphoria and perhaps eventually may lead to depression.

Finally, in Chapter 7 a general discussion of the results presented in the empirical chapters was given. 
Samenvatting 
Het centrale thema van dit proefschrift is visuo-spatiële selectieve aandacht. Selectieve aandacht helpt ons bij het geven van prioriteit aan relevante informatie en het tegelijkertijd negeren of onderdrukken van minder belangrijke input. Dit is nodig, omdat we dagelijks overspoeld worden door informatie, terwijl we in staat zijn hiervan slechts een beperkte hoeveelheid te verwerken. De term visuo-spatieel slaat op de visuele ruimte om ons heen. Samengevoegd gaat het bij visuo-spatiële aandacht dus om het verplaatsen van onze aandacht door de ruimte om ons heen. In de studies beschreven in dit proefschrift onderzochten we zowel visuo-spatiële selectieve aandacht an sich (Hoofdstukken 2-6), als ook de relatie tussen deze aandachtsprocessen en negatieve stemming (Hoofdstuk 5-6). We gebruikten verschillende versies van de endogene spatiële cueing taak (e.g. Posner, 1980). Om de relatie tussen aandacht en stemming te onderzoeken werd een dot-probe taak aangewend.

Visuo-spatiële aandacht kan overt gericht worden, door je ogen te bewegen in de richting van iets in je omgeving, maar ook covert, zonder dat je je ogen beweegt. In al onze studies waren we geïnteresseerd in coverte aandacht. In Hoofdstuk 6 werd ook overte aandacht onderzocht en werden er oogbewegingen (OBn) gemeten.

Naast reactietijden (RTn) en $\mathrm{OBn}$, maten we ook hersenactiviteit en wel door middel van het electroencefalogram (EEG; Hoofdstukken 2, 3, 6). EEG is een non-invasieve techniek waarmee het tijdverloop van cognitieve processen, zoals visuospatiële aandacht, in kaart gebracht kan worden met een milliseconden nauwkeurigheid. We leidden uit het EEG twee maten af: (1) gebeurtenis-gerelateerde potentialen (Event-Related Potentials, ERPs; Hoofdstukken 2, 3, 6) en (2) gebeurtenis-gerelateerde veranderingen in oscillaties (Event-Related Changes in Oscillations; Hoofdstukken 2, 6). Onze experimenten werden zo opgezet dat aandachtsverschuivingen zouden resulteren in verwerkingsverschillen tussen de hersenhelften. We waren dan ook vooral geïnteresseerd in gelateralizeerde maten: gelateralizeerde ERPs en gelateralizeerde veranderingen in oscillaties. Lateralizaties werden beschreven in termen van een positief of negatief voltage boven de hersenhelft contralateraal aan de richting van aandacht.

Hoofdstuk 1 omvat een theoretische introductie van de belangrijkste thema's die in dit proefschrift aan bod komen: (visuo-spatiële selectieve) aandacht, EEG maten (algemeen en in aandachtsonderzoek) en de relatie tussen selectieve aandacht en negatieve stemming in dysforie, depressie en bipolaire stoornis. Daarnaast worden in dit eerste hoofdstuk de doelstellingen van het onderzoek beschreven in dit proefschrift gepresenteerd: (1) Het afleiden van EEG maten die gevoelig zijn voor verschuivingen van selectieve visuele aandacht om hiermee het tijdverloop van aandacht in kaart te brengen, (2) Het bepalen van de funcionele betekenis van deze aandachtsmaten, (3) Het onderzoeken van aandachtsprocessen in situaties waarin aandacht niet gericht wordt door een voorafgaande directionele cue, (4) Het bestuderen van de rol van sequentie-effecten in een spatiële cueing taak, (5) Het onderzoeken van de relatie tussen aandachtsprocessen en negatieve stemming, (6) Het onderzoeken van de rol van verstoorde aandacht (aandachtsbias) in bipolaire stoornis en dysforie, (7) Het bestuderen van het tijdverloop van aandachtbias in geïnduceerde en spontaan ontstane dysforie. Er werden vijf studies uitgevoerd om bovenstaande thema's nader te onderzoeken. In de studies beschreven in Hoofdstukken 2 tot en met 4 staat het meten van aandacht door middel van EEG en RTn centraal; in Hoofdstukken 5 en 6 aandachtsbias in bipolaire stoornis en dysforie. 
In de studie beschreven in Hoofdstuk 2 voerden de deelnemers een endogene spatiële cueing taak uit. Tijdens elke trial werd ofwel een directionele pijl gepresenteerd die naar links $(<<)$ of naar rechts $(>>)$ wees, ofwel een dubbele, "neutrale" pijl die beiden kanten op wees $(<>)$. Deelnemers kregen de instructie om door middel van een knopdruk zo snel mogelijk een doelstimulus te detecteren die volgde na de pijl. De doelstimulus verscheen met een grotere waarschijnlijkheid aan de kant aangewezen door de directionele pijl dan aan de andere kant; neutrale pijlen hadden geen voorspellende waarde. De hoge voorspellende waarde van de directionele pijlen moest deelnemers motiveren hun aandacht te verschuiven in de richting van de pijl. Het hoofddoel van deze studie was om aandachtsprocessen te onderzoeken wanneer een neutrale pijl werd aangeboden. We hoopten de richting van aandacht te kunnen bepalen op basis van de EEG respons meteen na het verschijnen van de pijl. Om dit doel te bereiken was het noodzakelijk om maten die gevoelig zijn voor aandachtsverschuivingen af te leiden uit het EEG. Dit gebeurde in drie stappen.

Stap 1. We bestudeerden het EEG in respons op directionele pijlen: (1) ERP en (2) veranderingen in oscillaties. (1) De gemiddelde ERP na presentatie van de pijlen liet een opeenvolging zien van gelateralizeerde componenten die de resultaten van eerdere onderzoeken repliceerden (e.g. Eimer \& Van Velzen, 2002; Harter, Miller, Price, LaLonde, \& Keyes, 1989; Hopf \& Mangun, 2000; Nobre, Sebestyen, \& Miniussi, 2000). Een vroeger posterieure contralaterale positieve component tussen 150 en $250 \mathrm{~ms}$ werd gevolgd door een gelateralizeerde negativiteit tussen 250 en 500 ms contralateraal aan de richting van aandacht. Deze negativiteit representeerde respectievelijk een "early directional attention negativity" (EDAN) op posterieure elektroden en een "anterior directing attention negativity" (ADAN) op anterieure en centrale elektroden. Op posterieure elektroden werd de negativiteit gevolgd door een contralaterale positiviteit tussen 500 en 1200 ms die een "late directing attention positivity" (LDAP) representeerde. Bovenstaande gelateralizeerde componenten zouden verschillende stadia in de controle van aandacht reflecteren (zie Hoofdstuk 3). (2) Er waren gelateralizeerde veranderingen in oscillaties in de lagere alfa1 band (La1, 6-8 Hz), maar niet in de klassieke alfa band (8-12 Hz; Worden, Foxe, Wang, \& Simpson, 2000), of in hogere frequentie banden (Gruber, Müiller, Keil, \& Elbert, 1999).

Stap 2. Het RT validiteitseffect gebaseerd op de richting van de directionele pijl werd vergeleken met het validiteitseffect gebaseerd op de richting van aandacht zoals "voorspeld" op basis van de EEG respons. RTn dienden om te verifiëren dat er inderdaad selectieve aandachtsverschuivingen hadden plaatsgevonden. (1) ERP lateralizaties werden succesvol gebruikt om de richting van aandacht te voorspellen in afzonderlijke trials met directionele pijlen. (2) de voorspelling van de richting van aandacht op basis van veranderingen in de La1-band bleek niet succesvol. De gelateralizeerde ERP respons, opgebouwd uit de eerder genoemde componenten, kan dus dienen als valide maat voor de richting van aandacht. Deze classificatie-methode zou in de toekomst waardevol kunnen zijn in experimenten waarbij de afhankelijke variabele bestaat uit twee categorieën die onderscheiden kunnen worden op basis van de ERP respons.

Stap 3. We gebruikten gelateralizeerde ERP componenten om te bepalen of aandacht zich ook na een neutrale, in plaats van directionele, pijl spatieel verplaatst. Neutrale trials werden daartoe op basis van de ERP lateralizaties geclassificeerd als "aandacht naar links" of "aandacht naar rechts". RT analyse toonde geen validiteits-effect 
en daarmee geen bewijs dat aandacht in neutrale trials verschuift na de presentatie van een neutrale pijl. Een analyse van niet-gelateralizeerde EEG activiteit (alfa activiteit en $\mathrm{CNV}$ ) tussen het verdwijnen van de neutrale pijl en het verschijnen van de doelstimulus liet echter zien dat variaties in RTn tussen neutrale trials samenhingen met variaties in non-spatiële aandacht (algemene alertheid) en daarom niet enkel betekenisloze ruis reflecteerden.

Het doel van Hoofdstuk 3 was om ERP componenten gerelateerd aan sensorische aspecten van de pijl te onderscheiden van componenten gerelateerd aan verschuiving van aandacht. Dit werd gedaan door middel van een manipulatie van taakinstructie in twee endogene spatiële cueing taken. In de ene taak werden de fysieke kenmerken van pijlen $(<<,>>)$ gebalanceerd over aandacht-links en aandacht-rechts instructies. Dit resulteerde in een congruente conditie $(<<$ voor links, $>>$ voor rechts) en een incongruente conditie $(<<$ voor rechts, $>>$ voor links). Een vergelijking van deze twee condities zou aantonen welke ERP componenten gerelateerd zijn aan de fysieke vorm van de pijlen en welke aan de richting van aandacht. In de andere taak werden, in plaats van pijlen, niet-gelateralizeerde, verticale lijnen gebruikt waarvan de lengte de richting van aandacht bepaalde (bijv. "|" voor links, "|" voor rechts). Dit resulteerde in een conditie met een willekeurige relatie tussen lijnlengte en de geïnstrueerde richting van aandacht. Met deze lijn-stimuli zou de mogelijkheid kunnen worden uitgesloten dat gelateralizeerde ERP activiteit na presentatie van de lijn het gevolg zou zijn van gelateralizeerde aspecten van vorm, omdat deze simpelweg ontbraken.

Beide taken werden gebruikt in twee experimenten die verschilden in taakinstructie. In het eerste experiment werden deelnemers geïnstrueerd te reageren op elke perifeer aangeboden stimulus, ongeacht of deze verscheen op de plaats waar de voorafgaande cue naar wees (valide) of op de andere positie (invalide). In het tweede experiment werden deelnemers geïnstrueerd enkel te reageren op valide gecuede doelstimuli die perifeer werden gepresenteerd in slechts een klein deel van de trials.

Onze resultaten leverden een replicatie van de resultaten van eerdere studies (e.g. Eimer, 1994; Mangun \& Hillyard, 1991). Ze lieten namelijk zien dat modulatie door aandacht van vroege sensorische componenten na perifere stimuli afhing van taakinstructie, afhing van de relevantie van de niet-gecuede positie (d.w.z., of deze om een reactie vraagt of niet) en sterker was in het tweede experiment. We focusten op dezelfde gelateralizeerde ERP componenten als in Hoofdstuk 2: (1) posterieure contralaterale positieve component tussen 150 en 250 ms, (2) ADAN, LDAP, en (3) EDAN. (1) We vonden dat de vroege posterieure contralaterale positiviteit gerelateerd was aan de fysieke vorm van de pijlen. (2) De ADAN en LDAP daarentegen waren onafhankelijk van de fysieke vorm van cues en traden ook op wanneer lijnen in plaats van pijlen gebruikt werden als cues. Deze bevindingen tonen aan dat de ADAN en LDAP gerelateerd zijn aan de richting van aandacht. Beide componenten waren echter kleiner of zelfs afwezig wanneer modulatie van vroege sensorische componenten na perifere stimuli verminderd of afwezig was (experiment 1). Dit doet vermoeden dat er een relatie is tussen deze ERP componenten na presentatie van de cues en de sensorische modulatie na presentatie van de perifeer gepresenteerde stimuli. De ADAN liet verder net als in een eerdere studie (Eimer \& Van Velzen, 2002) een vertraging zien in die condities waar vertaling van de cue in de richting van aandacht complexer was, namelijk in de incongruente pijlen de arbitraire lijn-condities. (3) De data deden verder vermoeden dat de LDAP en de 
EDAN overlappen in de tijd en wat betreft locatie. Dit zou kunnen verklaren waarom de EDAN in bepaalde condities niet optrad. De EDAN trad alleen op wanneer pijlen en nooit wanneer lijnen gebruikt werden. Daarnaast was er geen bewijs dat de EDAN zuiver gerelateerd was aan de fysieke vorm van de pijl. Hieruit werd geconcludeerd dat de EDAN wellicht een reflectie is van de encodering van directionele informatie van pijlen en misschien ook andersoortige gelateralizeerde stimuli.

In Hoofdstuk 4 werden de effecten van verschillende opeenvolgingen van condities (sequentie-effecten) in een endogene spatiële cueing taak bestudeerd. Het paradigma was vergelijkbaar met dat uit Hoofdstuk 2. Alle mogelijke sequenties werden nu echter gebalanceerd aangeboden, wat leidde tot een fikse toename in het aantal trials. Het effect van de vorige cue richting, het vorige type trial (valide, invalide, neutraal, catch) en target alternatie werd onderzocht. De resultaten waren als volgt: (1) Zowel RT kosten als RT baten waren groter wanneer deze trials volgden op een valide trial dan wanneer ze volgden op een invalide trial. Deze bevinding veronderstelt dat deelnemers het gebruik van cues strategisch konden aanpassen: tijdens trials volgend op een invalide trial richtten zij hun aandacht minder sterk. (2) Na catch trials trad er een algehele vertraging op (Alegria, 1978; Correa, Lupiáñez, Milliken, \& Tudela, 2004; Los, 2004), terwijl kosten en baten van aandacht niet veranderden ten opzichte van trials volgend op een niet-catch trial. Deze bevinding ondersteunt het idee dat mechanismen die betrokken zijn bij het richten van aandacht onafhankelijk fungeren van een mechanisme van algemene alertheid (Posner \& Boies, 1971; Posner \& Petersen, 1990). (3) Tijdens neutrale trials werd het richten van aandacht beïnvloed werd door de richting van aandacht in de vorige trial. De positie die in de vorige trial gecued was, werd in de daaropvolgende trial onderdrukt. Deze bevinding werd gezien als bewijs dat endogene aandachtsverschuivingen resulteren in lange-termijn inhiberende processen die onafhankelijk zijn van exogene effecten uitgelokt door de voorafgaande perifere doelstimulus en van de validiteit van de vorige trial. Deze onafhankelijkheid is bijzonder, omdat aandachtsverschuivingen in de richting van de doelstimulus in de vorige trial recenter plaatsvonden dan aandachtsverschuivingen in reactie op de cue in de vorige trial. Deze bevinding ondersteunt het idee dat endogene en exogene aandachtseffecten onafhankelijk en additief kunnen zijn (e.g., Berger, Henik, \& Rafal, 2005; Berlucchi, Chelazzi, \& Tassinari, 2000).

In Hoofdstuk 5 werden zowel algemene visuo-spatiële aandachtsprocessen als aandachtsporcessen voor emotioneel beladen woorden (aandachtbias) onderzocht bij patiënten met bipolaire stoornis. De taak die gebruikt werd bestond uit een combinatie van een endogene spatiële cueing taak (zie Hoofdstuk 2) en een dot-probe taak. Net als in een standaard endogene spatiële cueing taak werd tijdens elke trial een pijl gepresenteerd op centrale fixatie: een predictieve, directionele pijl $(<<$ of $>>$ ) of een niet-predictieve, neutrale pijl $(<>)$. Net als in een standaard dot-probe taak werd tijdens elke trial een woordpaar gepresenteerd, een woord links en een woord rechts van centrale fixatie. In onze combinatietaak, werd het woordpaar tegelijkertijd met een centrale pijlcue gepresenteerd. Tijdens relevante trials werd een depressie-gerelateerd of een positief woord aangeboden samen met een emotioneel neutraal woord. De verschillende woordparen werden op zo'n manier gecombineerd met de verschillende pijlen dat algemene visuo-spatiële aandachtsprocessen en aandachtbias voor emotionele woorden onafhankelijk van elkaar gemeten konden worden in één en dezelfde taak. De 
deelnemers bestonden uit bipolaire patiënten in herstelde staat, bipolaire patiënten in een milde depressieve staat en niet-psychiatrische controles.

De resultaten op de endogene cueing taak waren als volgt: (1) Alle drie de groepen waren in staat hun aandacht te richten, zoals bleek uit significante RT baten van aandacht. (2) In de groep van bipolaire patiënten bleek een depressieve stemming positief gerelateerd aan RT kosten. De kosten vormen een reflectie van het relatieve onvermogen om aandacht los te koppelen.

De dot-probe resultaten waren als volgt: (1) Mild depressieve patiënten richtten hun aandacht weg van zowel depressie-gerelateerde als van positieve woorden, in vergelijking met de controle-groep. De resultaten vormden dus geen bewijs voor een specifieke, stemmingscongruente aandachtsbias in de richting van negatieve woorden in deze groep. (2) De aandachtbias weg van depressie-gerelateerde woorden was toestand-afhankelijk en correleerde binnen de patiëntengroep met depressieve stemming. (3) De aandachtsbias weg van positieve woorden was in beide patiëntengroepen even sterk aanwezig en was deel van een relatief stabiel ("trait"-) effect, kenmerkend voor patiënten. Dit laatste werd gedemonstreerd door herstelde patiënten te vergelijken met controles. De effecten van aandachtsbias gevonden voor depressie-gerelateerde en positieve woorden zouden wel eens gerelateerd kunnen zijn aan de overgang tussen verschillende stemmingstoestanden die zo karakteristiek zijn voor bipolaire stoornis. Een bias weg van alle emotionele informatie in de mild depressieven zou in dat geval een overgang voorspellen naar een herstelde staat. Een bias weg van positieve woorden en in de richting van depressie-gerelateerde woorden zou een overgang voorspellen van de herstelde staat naar een van de andere stemmingstoestanden.

In Hoofdstuk 6 werden zowel algemene visuo-spatiële aandachtsprocessen als aandachtsbias voor emotionele woorden onderzocht in dysforie (spontaan ontstaan of geïnduceerd in het lab) met behulp van de combinatietaak die gebruikt werd in Hoofdstuk 5 . Het tijdverloop van deze processen werd onderzocht met behulp van metingen van oogbewegingen en EEG na presentatie van respectievelijk pijlcues en woordstimuli. Net als in Hoofdstuk 2 werden gelateralizeerde ERPs en veranderingen in oscillaties in de La1-band afgeleid uit het EEG. Bij aankomst in het lab varieerde de stemming van de deelnemers van gezond tot dysfoor (niet-geïnduceerde dysforie). Bij de ene helft van de deelnemers werd door middel van een depressieve stemmings-inductie procedure (SIP) een depressieve stemming geïnduceerd (geïnduceerde dysforie), bij de andere helft werd door middel van een neutrale SIP een neutrale stemming geïnduceerd.

De resultaten op de endogene cueing taak waren als volgt: (1) Alle deelnemers waren in staat hun aandacht te richten, zoals bleek uit significante RT baten van aandacht. (2) In de depressieve SIP groep bleken de RT kosten van aandacht, die een reflectie vormen van het relatieve onvermogen om aandacht los te koppelen, hoger dan in de neutrale SIP groep. Deze bevinding is vergelijkbaar met de relatie tussen de depressieve stemming en RT kosten in Hoofdstuk 5. Deze data doen vermoeden dat negatieve stemming gerelateerd is aan een algemeen probleem om aandacht los te koppelen. (3) ERP resultaten en resultaten van gelateralizeerde veranderingen in de frequentieband repliceerden gedeeltelijk de resultaten van Hoofdstuk 2.

De dot-probe resultaten varieerden sterk in de tijd, zowel (1) tussen taak-blokken als (2) binnen trials. (1) Wanneer alle taak-blokken werden gemiddeld, waren er geen significante verschillen in aandachtsbias in RTn. Variantie van bias effecten over de tijd 
zouden ten grondslag kunnen liggen aan de heterogene resultaten van aandachtbias in depressie en dysforie. Onze resultaten laten zien dat het erg belangrijk is om de factor "taak-blok" op te nemen in het statistisch design wanneer blokken trials herhaaldelijk werden aangeboden. (2) Metingen van oogbewegingen $(\mathrm{OBn})$ in combinatie met RT metingen verschaften veel informatie over het tijdverloop van aandachtsbias binnen trials, zowel bij (a) niet-geïnduceerde dysforie als bij (b) geïnduceerde dysforie. De dotprobe resultaten leverden net als de resultaten beschreven in Hoofdstuk 5 weinig of geen bewijs voor een stemmingscongruente aandachtsbias in dysforie. (a) Metingen van OBn lieten zien dat niet-geïnduceerde dysforie gerelateerd was aan een bias weg van alle emotionele woorden, meteen na hun verschijnen. Metingen van RTn lieten zien dat deze vroege aandachtsbias gevolgd werd door een bias in de richting van depressiegerelateerde woorden of de afwezigheid van een bias voor deze woorden (in een aantal blokken) en een algehele afwezigheid van een bias voor positieve woorden (in alle blokken). (b) Metingen van OBn lieten zien dat de depressieve SIP groep, in vergelijking met de neutrale SIP groep, een vroege aandachtsbias liet zien weg van depressiegerelateerde woorden, maar in de richting van positieve woorden. Metingen van RTn lieten zien dat dit patroon van biases ofwel aanhield, ook na het verschijnen van de doelstimulus, ofwel gevolgd werd door de afwezigheid van bias (in enkele blokken).

Het patroon van biases dat te zien was in geïnduceerde dysforie werd al eerder gevonden (Ellenbogen, Schwartzman, Stewart, \& Walker, 2002; Miranda, Gross, Persons, Hahn, 1998) en onze resultaten vormen een aanvulling omdat ze laten zien dat het effect al vroeg in de tijd optreedt. Het zou een adaptief mechanisme kunnen reflecteren dat dient om emotie te reguleren. We speculeerden dat de vroege vermijdende bias, weg van alle emotionele woorden, in niet-geïnduceerde dysforie op vergelijkbare wijze zou kunnen dienen om emotionele arousal te reguleren en te verminderen in degenen die hoog scoorden op dysforie. In een deel van de taak hield dit mechanisme echter geen stand en verschoven dysforen hun aandacht richting depressie gerelateerde woorden. Dit falende regulerende mechanisme zou een van de cognitieve gevoeligheden kunnen zijn die karakteristiek zijn voor dysforie en uiteindelijk leiden tot depressie.

Hoofdstuk 7, ten slotte, omvat een algemene discussie van de resultaten als beschreven in de voorafgaande empirische hoofdstukken. 
88 chapter 9 


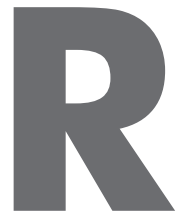

\section{References}




\section{REFERENCES}

Albersnagel, F. A. (1988). Velten and Musical Mood Induction Procedures: A comparison with accessibility of thought associations. Behaviour Research and Therapy, 26, 79-96.

Alegria, J. (1978). Sequential effects of catch-trials on choice reaction time. Acta Psychologica, 42, 1-6.

Alloy, L. B., \& Abramson, L. Y. (1988). Depressive realism: Four theoretical perspectives. In L. B. Alloy (Ed.), Cognitive processes in depression. New York: Guilford Press.

Anllo Vento, L., Schoenfeld, M. A., \& Hillyard, S. A. (2004). Cortical Mechanisms of Visual Attention: Electrophysiological and Neuroimaging Studies. In M. I. Posner (Ed.), Cognitive neuroscience of attention (pp. 180-193). New York: Guilford Press.

Awh, E., Armstrong, K.M., \& Moore, T. Visual and oculomotor selection: Links, causes and implications for spatial attention. Trends in Cognitive Sciences, 10, 124-130.

Baaijen, R. H., Piepenbrock, R., \& Gulikers, L. The CELEX Lexical Database (CD-ROM). Linguistic Data Consortium, University of Pennsylvania, Philadelphia, PA, 1995.

Babiloni, C., Miniussi, C., Babiloni, F., Carducci, F., Cincotti, F., Del Percio, C., et al. (2004). Sub-second «temporal attention» modulates alpha rhythms. A high resolution EEG study. Cognitive Brain Research, 19, 259-268.

Bar-Haim, Y., Lamy, D., \& Glickman, S. (2005). Attentional bias in anxiety: A behavioral and ERP study. Brain and Cognition, 59, 11-22.

Bastiaansen, M. C., \& Brunia, C. H. (2001). Anticipatory attention: an event-related desynchronization approach. International Journal of Psychophysiology, 43, 91-107.

Bastiaansen, M. C. M., Boecker, K. B. E., \& Brunia, C. H. M. (2002). ERD as an index of anticipatory attention? Effects of stimulus degradation. Psychophysiology, 39, 16-28.

Beck, A. T. (1976). Cognitive therapy and emotional disorders. New York: New American Library.

Beck, A. T. (1987). Cognitive models of depression. Journal of Cognitive Psychotherapy: An International Quarterly, 1, 5-37.

Beck, A. T., Steer, R. A., \& Garbin, M. G. (1988). Psychometric properties of the Beck Depression Inventory: twenty-five years of evaluation. Clinical Psychology Review, 8, 77-100.

Beck, A. T., Ward, C. H., Mendelson, M., Mock, J., \& Erbaugh, J. (1961). An inventory for measuring depression. Archives of General Psychiatry, 4, 561-571.

Berger, A., Henik, A., \& Rafal, R. D. (2005). Competition between endogenous and exogenous orienting of visual attention. Journal of Experimental Psychology: General, 134, 207-221.

Beringer, J. (1987). Experimental Run Time System (Version 3.32c). Frankfurt, Germany: Berisoft Cooperation.

Berlucchi, G. (2006). Inhibition of return: A phenomenon in search of a mechanism and a better name. Cognitive Neuropsychology, 23, 1065-1074.

Berlucchi, G., Chelazzi, L., \& Tassinari, G. (2000). Volitional covert orienting to a peripheral cue does not suppress cue-induced inhibition of return. Journal of Cognitive Neuroscience, 12, 648-663.

Bertelson, P. (1961). Sequential redundancy and speed in a serial two-choice responding task. Quarterly Journal of Experimental Psychology, 13, 90-102. 
Bertelson, P. (1963). S-R relationships and reaction times to new versus repeated signals in a serial task. Journal of Experimental Psychology, 65, 478-484.

Bertelson, P. (1967). The time course of preparation. Quarterly Journal of Experimental Psychology, 19, 272-279.

Bertelson, P., \& Renkin, A. (1966). Reaction times to new versus repeated signals in a serial task as a function of response-signal time interval. Acta Psychologica, 25, 132136.

Boiten, F., Sergeant, J., \& Geuze, R. (1992). Event-related desynchronization: the effects of energetic and computational demands. Electroencephalography and Clinical Neurophysiology, 82, 302-309.

Bouyer, J. J., Montarom, M. F., Vahnee, J. M., Albert, M. P., \& Rougeul, A. (1987). Anatomical localization of cortical beta rhythms in cat. Neuroscience, 22, 863-869.

Bower, G. H. (1981). Mood and memory. American Psychologist, 36, 129-148.

Bradley, B. P., \& Mathews, A. (1983). Negative self-schemata in clinical depression. British journal of clinical psychology, 22, 173-181.

Bradley, B. P., Mogg, K., Falla, S. J., \& Hamilton, L. R. (1998). Attentional bias for threatening facial expressions in anxiety: Manipulation of stimulus duration. Cognition and Emotion, 12, 737-753.

Bradley, B. P., Mogg, K., Lee, S. C. (1997). Attentional biases for negative information in induced and naturally occurring dysphoria. Behaviour Research and Therapy, 35, 911-927.

Bradley, B. P., Mogg, K., \& Millar, N. H. (2000). Covert and overt orienting of attention to emotional faces in anxiety. Cognition and Emotion, 14, 789-808.

Bradley, B. P., Mogg, K., Millar, N., Bonham Carter, C., \& et al. (1997). Attentional biases for emotional faces. Cognition and Emotion, 11, 25-42.

Bradley, B. P., Mogg, K., Millar, N., \& White, J. (1995). Selective processing of negative information: Effects of clinical anxiety, concurrent depression, and awareness. Journal of Abnormal Psychology, 104, 532-536.

Bradley, B. P., Mogg, K., White, J., Groom, C., \& de Bono, J. (1999). Attentional bias for emotional faces in generalized anxiety disorder. British Journal of Clinical Psychology, 38, 267-278.

Brunia, C. H., \& Damen, E. J. (1988). Distribution of slow brain potentials related to motor preparation and stimulus anticipation in a time estimation task. Electroencephalography and Clinical Neurophysiology, 69, 234-243.

Brunia, C. H. M., \& van Boxtel, G. J. M. (2001). Wait and see. International Journal of Psychophysiology., 43, 59-75.

Cheal, M., \& Chaistain, G. (1999). Inhibition of return: Support for generality of the phenomenon. Journal of General Psychology, 126, 375-390.

Clark, D. A., \& Beck, A. T. (1999). Scientific foundations of cognitive therapy and therapy of depression. New York: John Wiley \& Sons, Inc.

Clark, C.R., Geffen, G.M., \& Geffen, L.B. (1989). Catecholamines and the covert orientation of attention in humans. Neuropsychologia, 27, 131-139.

Clark, D. M., \& Teasdale, J. D. (1985). Constraints on the effects of mood on memory. Journal of Personality and Social Psychology, 48, 1595-1608.

Clohessy, A.B., Posner, M.I., Rothbart, M.K., \& Vecera, S.P. (1991).The development of inhibition of return in early infancy, Journal of Cognitive Neuroscience, 3, 345-350. 
Compton, R. J. (2000). Ability to disengage attention predicts negative affect. Cognition and Emotion, 14, 401-415.

Corbetta, M., \& Shulman, G. L. (2002). Control of goal-directed and stimulus-driven attention in the brain. Nature Reviews Neuroscience, 3, 201-215.

Correa, A., Lupiáñez, J., Milliken, B., \& Tudela, P. (2004). Endogenous temporal orienting of attention in detection and discrimination tasks. Perception and Psychophysics, 66, 264-278.

Coull, J. T., \& Nobre, A. C. (1998). Where and when to pay attention: The neural systems for directing attention to spatial locations and to time intervals as revealed by both PET and AMRI. Journal of Neuroscience, 18, 7426-7435.

Cuellar, A. K., Johnson, S. L., \& Winters, R. (2005). Distinctions between bipolar and unipolar depression. Clinical Psychology Review, 25, 307-339.

Dalgleish, T., \& Watts, F. N. (1990). Biases of attention and memory in disorders of anxiety and depression. Clinical Psychology Review, 10, 589-604.

Damen, E.J.P., \& Brunia, C.H.M. (1987). Precentral potential shifts related to motor preparationand stimulus anticipation: a replication. In R. Johnson Jr., J.W. Rohrbaugh and R. Parasuraman (Eds.), Current Trends in event-related potential research (pp. 1316). New York, Elsevier.

De Gonzaga Gawryszewski, L., Riggio, L., Rizzolatti, G., \& Umilta, C. (1987). Movements of attention in the three spatial dimensions and the meaning of "neutral" cues. Neuropsychologia, 25, 19-29.

Deldin, P.J., Keller, J., Gergen, J.A., Miller, G.A. (2001). Cognitive bias and emotion in neuropsychological models of depression. Cognition \& Emotion, 115, 787-802.

Dell'Acqua, R., Pesciarelli, F., Jolicoeur, P., Eimer, M., \& Peressotti, F. (2006). The interdependence of spatial attention and lexical access as revealed by early asymmetries in occipito-parietal ERP activity. Manuscript submitted for publication.

De Pascalis, V., \& Ray, W. J. (1998). Effects of memory load on event-related patterns of 40-Hz EEG during cognitive and motor tasks. International Journal of Psychophysiology, 28, 301-315.

Derryberry, D., \& Reed, M. A. (2002). Anxiety-related attentional biases and their regulation by attentional control. Journal of Abnormal Psychology, 111, 225-236.

Derryberry, D., \& Rothbart, M. K. (1988). Arousal, affect, and attention as components of temperament. Journal of Personality and Social Psychology, 55, 958-966.

Derryberry, D., \& Rothbart, M. K. (1997). Reactive and effortful processes in the organization of temperament. Development and Psychopathology, 9, 633-652.

De Ruiter, C., \& Brosschot, J. F. (1994). The emotional Stroop effect in anxiety: Attentional bias or cognitive avoidance? Behaviour Research and Therapy, 32, 315-319.

Deubel, H., \& Schneider, W.A. (1996). Saccade target selection and object recognition: evidence for a common attentional mechanism. Vision Research, 36, 1827-1837.

Doppelmayr, M., Klimesch, W., Pachinger, T., \& Ripper, B. (1998). Individual differences in brain dynamics: important implications for the calculation of event-related band power. Biological Cybernetics, 79, 49-57.

Downing, C. J. (1988). Expectancy and visual-spatial attention: effects on perceptual quality. Journal of Experimental Psychology: Human Perception and Performance, 14, 188-202. 
Dreisbach, G., \& Goschke, T. (2004). How positive affect modulates cognitive control: Reduced perseveration at the cost of increased distractibility. Journal of Experimental Psychology: Learning, Memory, and Cognition, 30, 343-353.

Driver, J., Eimer, M., Macaluso, E., \& J. Van Velzen (2004). The neurobiology of human spatial attention. In N. Kanwisher \& J. Duncan (Eds.), Imaging visual cognition: Attention and Performance XX. (pp. 267-300). Oxford: OUP.

Dujardin, K., Derambure, P., Defebvre, L., Bourriez, J. L., Jacquesson, J. M., \& Guieu, J. D. (1993). Evaluation of event-related desynchronization (ERD) during a recognition task: effect of attention. Electroencephalography and Clinical Neurophysiology, 86, 353-356.

Eason, R.G., Harter, R.G., \& White, C. (1969). Effects of attention and arousal on visually evoked potentials and reaction time in man. Physiology and Behavior, 4, 283-289.

Eimer, M. (1993). Spatial cueing, sensory gating and selective response preparation: An ERP study on visuo-spatial orienting. Electroencephalography and Clinical Neurophysiology: Evoked Potentials, 88, 408-420.

Eimer, M. (1994). "Sensory gating” as a mechanism for visuospatial orienting: Electrophysiological evidence from trial-by-trial cuing experiments. Perception and Psychophysics, 55, 667-675.

Eimer, M. (1996). The N2pc component as an indicator of attentional selectivity. Electroencephalography and Clinical Neurophysiology, 99, 225-234.

Eimer, M. (1997). Uninformative symbolic cues may bias visual-spatial attention: behavioral and electrophysiological evidence. Biological Psychology, 46, 67-71.

Eimer, M., \& Van Velzen, J. (2002). Crossmodal links in spatial attention are mediated by supramodal control processes: evidence from event-related potentials. Psychophysiology, 39, 437-449.

Eimer, M., van Velzen, J., \& Driver, J. (2002). Cross-modal interactions between audition, touch, and vision in endogenous spatial attention: ERP evidence on preparatory states and sensory modulations. Journal of Cognitive Neuroscience, 14, 254-271.

Eizenman, M., Yu, L. H., Grupp, L., Eizenman, E., Ellenbogen, M. A., Gemar, M., et al. (2003). A naturalistic visual scanning approach to assess selective attention in major depressive disorder. Psychiatry Research, 118, 117-128.

Ellenbogen, M. A., Schwartzman, A. E., Stewart, J., \& Walker, C. D. (2002). Stress and selective attention: The interplay of mood, cortisol levels, and emotional information processing. Psychophysiology, 39, 723-732.

Eriksen, B. A., \& Eriksen, C. W. (1974). Effects of noise letters upon the identification of a target letter in a nonsearch task. Perception and Psychophysics, 16, 143-149.

Estes, W. K. (1964). Probability learning. In A. W. Melton (Ed.), Categories of human learning (pp. 89-128). New York: Academic Press.

Fernandez-Duque, D., \& Posner, M.I. (1997). Relating the mechanisms of orienting and alerting. Neuropsychologia, 35, 477-486.

Filipovic, S. R., Jahanshahi, M., \& Rothwell, J. C. (2001). Uncoupling of contingent negative variation and alpha band event-related desynchronization in a go/no-go task. Clinical Neurophysiology, 112, 1307-1315.

Fink, A. (2005). Event-related desynchronization in the EEG during emotional and cognitive information processing: Differential effects of extraversion. Biological Psychology, 70, 152-160. 
Fox, E., Russo, R., Bowles, R., \& Dutton, K. (2001). Do threatening stimuli draw or hold visual attention in subclinical anxiety? Journal of Experimental Psychology: General, 130, 681-700.

Foxe, J. J., Simpson, G. V., \& Ahlfors, S. P. (1998). Parieto-occipital-10 Hz activity reflects anticipatory state of visual attention mechanisms. Neuroreport: An International Journal for the Rapid Communication of Research in Neuroscience, 9, 3929-3933.

Fu, K. M. G., Foxe, J. J., Murray, M. M., Higgins, B. A., Javitt, D. C., \& Schroeder, C. E. (2001). Attention-dependent suppression of distracter visual input can be cross-modally cued as indexed by anticipatory parieto-occipital alpha-band oscillations. Cognitive Brain Research, 12, 145-152.

Gal, I., \& Baron, J. (1996). Understanding repeated simple choices. Thinking and reasoning, 2, 81-98.

Girelli, M., \& Luck, S. J. (1997). Are the same attentional mechanisms used todetect visual search targets defined by color, orientation, and motion? Journal of Cognitive Neuroscience, 9, 238-253.

Goldman, R. I., Stern, J. M., Engel, J., Jr., \& Cohen, M. S. (2002). Simultaneous EEG and fMRI of the alpha rhythm. Neuroreport, 13, 2487-2492.

Gordon, I. E. (1967). Stimulus probability and simple reaction time. Nature, 215, 895-896.

Gotlib, I. H., Kasch, K. L., Traill, S., Joormann, J., Arnow, B. A., \& Johnson, S. L. (2004). Coherence and specificity of information-processing biases in depression and social phobia. Journal of Abnormal Psychology, 113, 386-398.

Gotlib, I. H., \& Krasnoperova, E. (1998). Biased information processing as a vulnerability factor for depression. Behavior Therapy., 29, 603-617.

Gotlib, I. H., Krasnoperova, E., Yue, D. N., \& Joormann, J. (2004). Attentional Biases for Negative Interpersonal Stimuli in Clinical Depression. Journal of Abnormal Psychology, 113, 127-135.

Gotlib, I. H., McLachlan, A. L., \& Katz, A. N. (1988). Biases in visual attention in depressed and nondepressed individuals. Cognition and Emotion, 2, 185-200.

Gratton, G. (1998). Dealing with artifacts: The EOG contamination of the eventrelated brain potential. Behavior Research Methods, Instruments and Computers, 30, 44-53.

Gratton, G., Coles, M. G., \& Donchin, E. (1992). Optimizing the use of information: Strategic control of activation of responses. Journal of Experimental Psychology: General, 121, 480-506.

Green, J. J., \& McDonald, J. J. (2006). An event-related potential study of supramodal attentional control and crossmodal attention effects. Psychophysiology, 43, 161-171.

Green, J. J., Teder Saelejaervi, W., \& McDonald, J. J. (2005). Control mechanisms mediating shifts of attention in auditory and visual space: a spatio-temporal ERP analysis. Experimental Brain Research, 166, 358-369.

Griffin, I. C., Miniussi, C., \& Nobre, A. C. (2002). Multiple mechanisms of selective attention: Differential modulation of stimulus processing by attention to space or time. Neuropsychologia, 40, 2325-2340.

Gross, J. J., \& Munoz, R. F. (1995). Emotion regulation and mental health. Clinical Psychology: Science and Practice, 2, 151-164. 
Gruber, T., Muller, M. M., Keil, A., \& Elbert, T. (1999). Selective visual-spatial attention alters induced gamma band responses in the human EEG. Clinical Neurophysiology, 110, 2074-2085.

Hackley, S. A., \& Miller, J. (1995). Response complexity and precue interval effects on the lateralized readiness potential. Psychophysiology, 32, 230-241.

Hamilton, M. (1960). A rating for depression. Journal of Neurology, Neurosurgery, and Psychiatry, 23, 56-61.

Handy, T. C., \& Mangun, G. R. (2000). Attention and spatial selection: Electrophysiological evidence for modulation by perceptual load. Perception and Psychophysics., 62, 175-186.

Harmer, C.J., Hill, S.A., Taylor, M.J., Cowen, P.J., \& Goodwin, G.M. (2003). Toward a neuropsychological theory of antidepressant drug action: Increase in positive emotional bias after potentiation of norepinephrine activity. American Journal of Psychoatry, 160, 990-992.

Harter, M. R., \& Anllo-Vento, L. (1991). Visual-spatial attention: preparation and selection in children and adults. Electroencephalography and Clinical Neurophysiology Supplement, 42, 183-194.

Harter, M. R., Miller, S. L., Price, N. J., LaLonde, M. E., \& Keyes, A.L. (1989). Neural processes involved in directing attention. Journal of Cognitive Neuroscience, 1, 223237.

Hermans, D., Vansteenwegen, D., \& Eelen, P. (1999). Eye movement registration as a continuous index of attention deployment: Data from a group of spider anxious students. Cognition and Emotion., 13, 419-434.

Hill, A. B., \& Duiton, F. (1989). Depression and selective attention to self-esteem threatening words. Personality and Individual Differences, 10, 915-917.

Holmes, A., Vuilleumier, P., \& Eimer, M. (2003). The processing of emotional facial expression is gated by spatial attention: evidence from event-related brain potentials. Cognitive Brain Research, 16, 174-184.

Hommel, B. (2004). Event files: feature binding in and across perception and action. Trends in Cognitive Sciences, 8, 494-500.

Hommel, B., Proctor, R.W., \& Vu, K.L. (2004). A feature-integration account of sequential effects in the Simon task. Psychological Research, 68, 1-17.

Hopf, J. M., \& Mangun, G. R. (2000). Shifting visual attention in space: an electrophysiological analysis using high spatial resolution mapping. Clinical Neurophysiology, 111, 1241-1257.

Howell, D. C. (2002). Statistical methods for psychology (5th ed.). Pacific Grove, CA.: Duxbury.

Hyman, R. (1953). Stimulus information as a determinant of reaction time. Journal of Experimental Psychology, 45, 188-196.

Jansson, B., \& Lundh, L. (2006). The interactive role of worried mood and trait anxiety in the selective processing of subliminally presented threat words. Personality and Individual Differences, 41, 1195-1204.

Jongen, E. M. M., \& Smulders, F. T. Y. (2005, September). Electrophysiological measurement of attentional bias in induced and naturally occurring dysphoria. Poster session presented at the IX International Conference on Cognitive Neuroscience, Havana, Cuba. 
Jongen, E. M. M., Smulders, F. T. Y., Ranson, S.M.G., Arts, B.M.G., \& Krabbendam, L. (in press). Attentional bias and general orienting processes in bipolar disorder. Journal of Behavioral Therapy and Experimental Psychiatry.

Jongen, E. M. M., Smulders, F. T. Y., \& Van Breukelen, G. J. P. (2006). Varieties of attention in neutral trials: Linking RT to ERPs and EEG frequencies. Psychophysiology, 43, 113-125.

Jongen, E. M. M., Smulders, F. T. Y., \& Van der Heiden (2007). Lateralized ERP components related to attentional orienting: Discriminating shifts of attention from sensory aspects of the cue. Manuscript in revision.

Jonides, J. (1981). Voluntary versus automatic control over the mind's eye's movement. In J. Long (Ed.), Attention and performance IX: 9th international symposium, Cambridge, July (Vol. IX, pp. 187-203). Hillsdale: Erlbaum.

Jonides, J. (1983). Further toward a model of the mind's eye's movement. Bulletin of the Psychonomic Society, 21, 247-250.

Jonides, J., \& Mack, R. (1984). On the cost and benefit of cost and benefit. Psychological Bulletin, 96, 29-44.

Joormann, J. (2004). Attentional bias in dysphoria: The role of inhibitory processes. Cognition and Emotion, 18, 125-147.

Juan, C.H., Shorter-Jacobi, S.M., Schall, J.D. (2004). Dissociation of spatial attention and saccade preparation. Proc Natl Acad Sci U S A, 101, 15541-15544.

Judd, L. L., Akiskal, H. S., Schettler, P. J., \& al., e. (2002). The long-term natural history of the weekly symptomatic status of nipolar I disorder. Archives of General Psychiatry, 59, 530-537.

Judd, L. L., Akiskal, H. S., Schettler, P. J., \& al., e. (2003). A prospective investigation of the natural history of the long-term weekly symptomatic status of bipolar II disorder. Archives of General Psychiatry, 60, 261-269.

Kalcher, J., \& Pfurtscheller, G. (1995). Discrimination between phase-locked and nonphase-locked event-related EEG activity. Electroencephalography and Clinical Neurophysiology, 94, 381-384.

Karparova, S.P., Kersting, A., Suslow, T. (2005). Disengagaement of attention from facial emotion in unipolar depression. Psychiatry and Clinical Neurosciences, 59, 723729.

Keil, A., Gruber, T., \& Muller, M. M. (2001). Functional correlates of macroscopic high-frequency brain activity in the human visual system. Neuroscience and Biobehavioral Reviews, 25, 527-534.

Kendall, P. C., Hollon, S. D., Beck, A. T., Hammen, C. L., \& Ingram, R. E. (1987). Issues and recommendations regarding use of the Beck Depression Inventory. Cognitive Therapy and Research, 11, 289-299.

Kennett, S., Van Velzen, J., Eimer, M., \& Driver, J. (2007). Disentangling gaze shifts from preparatory ERP effects during spatial attention. Psychophysiology, 44, 69-78.

Kerr, N., Scott, J, \& Phillips, M.L. (2005). Patterns of attentional deficits and emotional bias in bipolar and major depressive disorder. British Journal of Clinical Psychology, 44, 343-356.

Kirby, N. H. (1976). Sequential effects in two-choice reaction time: Automatic facilitation or subjective expectancy? Journal of Experimental Psychology: Human Perception and Performance, 2, 567-577. 
Kirby, N. H. (1980). Sequential effects in choice reaction time. In A. T. Welford (Ed.), Reaction times (pp. 129-172). London: Academic Press.

Klein, R. (1980). Does oculomotor readiness mediate cognitive control of visual attention? In R. Nickerson (Ed.), Attention and Performance. Hillsdale, NJ.: Lawrence Erlbaum.

Klein, R.M. (1988). Inhibitory tagging system facilitates visual search, Nature, 334, 430-431.

Klein, R.M. (2000). Inhibition of return. Trends in Cognitive Sciences, 4, 138-147.

Klein, R. M., Kingstone, A., \& Pontefract, A. (1992). Orienting of visual attention. In K. Rayner (Ed.), Eye movements and visual cognition: scene perception and reading (pp. 46-65). New York: Springer.

Kleinbaum, D. G., Kupper, L. L., Muller, K. E., \& Azhar, N. (1998). Applied regression analysis and multivariable methods (3rd ed.). Pacific Grove, CA: Brooks/Cole Publishing Company.

Klimesch, W. (1999). EEG alpha and theta oscillations reflect cognitive and memory performance: a review and analysis. Brain Research Reviews, 29, 169-195.

Klimesch, W., Schimke, H., Doppelmayr, M., Ripper, B., Schwaiger, J., \& Pfurtscheller, G. (1996). Event-related desynchronization (ERD) and the Dm effect: Does alpha desynchronization during encoding predict later recall performance? International Journal of Psychophysiology, 24, 47-60.

Klimesch, W., Doppelmayr, M., Russegger, H., Pachinger, T., \& Schwaiger, J. (1998a). Induced alpha band power changes in the human EEG and attention. Neuroscience Letters, 244, 73-76.

Klimesch, W., Russegger, H., Doppelmayr, M., \& Pachinger, T. (1998b). A method for the calculation of induced band power: implications for the significance of brain oscillations. Electroencephalography and Clinical Neurophysiology, 108, 123-130.

Koster, E. H. W., de Raedt, R., Goeleven, E., Franck, E., \& Crombez, G. (2005). Moodcongruent attentional bias in dysphoria: Maintained attention to and impaired disengagement from negative information. Emotion, 5, 446-455.

Koster, E. H. W., Leyman, L., de Raedt, R., \& Crombez, G. (2006). Cueing of visual attention by emotional facial expressions: The influence of individual differences in anxiety and depression. Personality and Individual Differences, 41, 329-339.

Koster, E. H. W., Verschuere, B., Crombez, G., \& van Damme, S. (2005). Time-course of attention for threatening pictures in high and low trait anxiety. Behaviour Research and Therapy, 43, 1087-1098.

Kowler, E. (in press). Attention and eye movements. In: The New Encyclopedia of Neuroscience. Krauzlis, R. (Ed.). Elsevier, Amsterdam.

Kowler, E., Anderson, E., Dosher, B., \& Blaser, E. (1995). The role of attention in the programming of saccades. Vision Research, 35, 1897-1916.

Kwak, H., \& Egeth, H. (1992). Consequences of allocating attention to locations and to other attributes. Perception and Psychophysics, 51, 455-464.

LaBerge, D., Carlson, R. L., Williams, J. K., \& Bunney, B. G. (1997). Shifting attention in visual space: Tests of moving-spotlight models versus an activity-distribution model. Journal of Experimental Psychology: Human Perception and Performance., 23, 13801392. 
Lavie, N. (1995). Perceptual load as a necessary condition for selective attention. Journal of Experimental Psychology: Human Perception and Performance, 21, 451-468.

Liu, X., Qian, M., Zhou, X., \& Wang, A. (2006). Repeating the stimulus exposure to investigate what happens after initial selective attention to threatening pictures. Personality and Individual Differences, 40, 1007-1016.

Los, S. (2004). Inhibition of return and non-specific preparation: separable inhibitory control mechanisms in space and time. Perception and Psychophysics, 66, 119-130.

Luck, S. J., Girelli, M., McDermott, M. T., \& Ford, M. A. (1997). Bridging the gap between monkey neurophysiology and human perception: an ambiguity resolution theory of visual selective attention. Cognitive Psychology, 33, 64-87.

Luck, S. J., \& Hillyard, S. A. (1994). Spatial filtering during visual search: evidence from human electrophysiology. Journal of Experimental Psychology: Human Perception and Performance, 20, 1000-1014.

Luck, S. J., Woodman, G. F., \& Vogel, E. K. (2000). Event-related potential studies of attention. Trends in Cognitive Sciences, 4, 432-440.

Lupiáñez, J., Klein, R.M., \& Bartolomeo, P. (2006). Inhibition of return: Twenty years after. Cognitive Neuropsychology, 23, 1003-1014.

Luteijn, F., \& van der Ploeg, F. A. E. (1983). Groninger Intelligentie Test (GIT) Handleiding [A manual for the Groninger Test of Intelligence]. Swets \& Zeitlinger b.v., Lisse.

Lyon, H. M., Startup, M., \& Bentall, R. P. (1999). Social cognition and the manic defense: Attributions, selective attention, and self-schema in bipolar affective disorder. Journal of Abnormal Psychology, 108, 273-282.

MacLeod, C. M. (1991). Half a century of research on the Stroop effect: An integrative review. Psychological Bulletin, 109, 163-203.

MacLeod, C., Mathews, A., \& Tata, P. (1986). Attentional bias in emotional disorders. Journal of Abnormal Psychology, 95, 15-20.

Malhi, G.S., Lagopoulos, J., Sachdev, P.S., Ivanovski, B., Shnier, R. (2005). An emotional Stroop functional MRI study of euthymic bipolar disorder. Bipolar Disorders, 7 , 58-69.

Mangun, G. R. (1994). Orienting attention in the visual fields: an electrophysiological analysis. In H. J. Heinze, T. F. Munte \& G. R. Mangun (Eds.), Cognitive electrophysiology (pp. 81-101). Boston, MA: Birkhauser.

Mangun, G.R., Hansen, G.R., \& Hillyard, S.A. (1986). The spatial orienting of attention: Sensory facilitation or response bias? In: R. Johnson Jr., J.W. Rohrbaugh, \& R. Parasuraman (Eds.), Current Trends in event-related potential research (pp.118-124). New York: Elsevier.

Mangun, G. R., \& Hillyard, S.A. (1990). Electrphysiological studies of visual selective attention in humans. In A. B. Scheibel, \& Wechsler, A.F. (Ed.), Neurobiology of higher cognitive function (pp. 271-294). London: The Guilford Press.

Mangun, G. R., \& Hillyard, S. A. (1991). Modulations of sensory-evoked brain potentials indicate changes in perceptual processing during visual-spatial priming. Journal of Experimental Psychology: Human Perception and Performance, 17, 1057-1074.

Mangun, G. R., Hillyard, S. A., \& Luck, S. J. (1993). Electrocortical substrates of visual selective attention. In D. E. Meyer \& S. Kornblum (Eds.), Attention and performance 14: Synergies in experimental psychology, artificial intelligence, and cognitive neuroscience. (pp. 219-243). Cambridge, MA, US: The MIT Press. 
Mansell, W., Clark, D. M., Ehlers, A., \& Chen, Y. P. (1999). Social anxiety and attention away from emotional faces. Cognition and Emotion, 13, 673-690.

Mansell, W., Colom, F., \& Scott, J. (2005). The nature and treatment of depression in bipolar disorder: A review and implications for future psychological investigation. Clinical Psychology Review, 25, 1076-1100.

Martin, M., Williams, R., \& Clark, D. (1991). Does anxiety lead to selective processing of threat-related information? Behaviour Research and Therapy, 29, 147-160.

Mathews, A. (1990). Why worry? The cognitive function of anxiety. Behaviour Research and Therapy., 28, 455-468.

Mathews, A., \& Mackintosh, B. (1998). A cognitive model of selective processing in anxiety. Cognitive Therapy and Research, 22, 539-560.

Mathews, A., \& MacLeod, C. (1985). Selective processing of threat cues in anxiety states. Behaviour Research and Therapy., 23, 563-569.

Mathews, A., \& MacLeod, C. (1994). Cognitive approaches to emotion and emotional disorders. Annual Review of Psychology, 45, 25-50.

Mathews, A. \& MacLeod, C. (2005). Cognitive vulnerability to emotional disorders. Annual Review of Clinical Psychology, 1, 167-195.

Mathews, A., Ridgeway, V., \& Williamson, D. A. (1996). Evidence for attention to threatening stimuli in depression. Behaviour Research and Therapy, 34, 695-705.

Matthews, G. R., \& Antes, J. R. (1992). Visual attention and depression: Cognitive biases in the eye fixations of the dysphoric and the nondepressed. Cognitive Therapy and Research., 16, 359-371.

Maylor, E. A., \& Hockey, R. (1987). Effects of repetition on the facilitatory and inhibitory components of orienting in visual space. Neuropsychologia., 25, 41-54.

McCabe, S. B., \& Gotlib, I. H. (1995). Selective attention and clinical depression: Performance on a deployment-of-attention task. Journal of Abnormal Psychology., 104, 241-245.

McCabe, S. B., \& Toman, P. E. (2000). Stimulus exposure duration in a deploymentof-attention task: Effects on dysphoric, recently dysphoric, and nondysphoric individuals. Cognition and Emotion, 14, 125-142.

McCracken, J., Osterhout, C., \& Voss, J. F. (1962). Effects of instructions in probability learning. Journal of Experimental Psychology, 64, 267-271.

McGuffin, P., Farmer, A., \& Harvey, I. (1991). A polydiagnostic application of the operational criteria in studies of psychotic illness. Development and reliability of the OPCRIT system. Archives of General Psychiatry, 48, 764-770.

McKenna, F.P., \& Sharma, D. (2004). Reversing the emotional Stroop effect reveals that it is not what it seems: the role of fast and slow components. Journal of Experimental Psychology, Learning, memory, and cognition, 30, 382-392.

McPeek, R.M., \& Keller, E.L. (2002). Saccade target selection in the superior colliculus during a visual search task. Journal of Neurophysiology, 88, 2019-2034.

McPeek, R.M., Maljkovic, V., \& Nakayama, K. (1999). Saccades require focal attention and are facilitated by a short-term memory system. Vision Research, 39, 1555-1566.

Melara, R. D., \& Algom, D. (2003). Driven by information: A tectonic theory of Stroop effects. Psychological Review, 110, 422-471.

Miller, G. A., \& Chapman, J.P. (2001). Misunderstanding analysis of covariance. Journal of Abnormal Psychology, 110, 40-48. 
Milliken, B., Lupiáñez, J., Roberts, M., \& Stevanovski, B. (2003). Orienting in space and time: Joint contributions to exogenous spatial cuing effects. Psychonomic Bulletin and Review, 10, 877-883.

Mineka, S., Watson, D., \& Clark, L. A. (1998). Comorbidity of anxiety and unipolar mood disorders. Annual Review of Psychology, 49, 377-412.

Miniussi, C., Wilding, E. L., Coull, J. T., \& Nobre, A. C. (1999). Orienting attention in time: Modulation of brain potentials. Brain, 122, 1507-1518.

Miranda, J., Gross, J. J., Persons, J. B., \& Hahn, J. (1998). Mood matters: negative mood induction activates dysfunctional attitudes in women vulnerable to depression. Cognitive Therapy and Research, 22, 363-376.

Miranda, J., \& Persons, J. B. (1988). Dysfunctional thoughts are mood-state dependent. Journal of Abnormal Psychology, 97, 76-79.

Mitchell, P. B., \& Malhi, G. S. (2004). Bipolar depression: phenomenological overview and clinical characteristics. Bipolar Disorders, 6, 530-539.

Mogg, K., \& Bradley, B. P. (2005). Attentional bias in generalized anxiety disorder versus depressive disorder. Cognitive Therapy and Research, 29, 29-45.

Mogg, K., Bradley, B. P., Miles, F., \& Dixon, R. (2004). Time course of attentional bias for threat scenes: Testing the vigilance-avoidance hypothesis. Cognition and Emotion, $18,689-700$.

Mogg, K., Bradley, B. P., \& Williams, R. (1995). Attentional bias in anxiety and depression: The role of awareness. British Journal of Clinical Psychology., 34, 17-36.

Mogg, K., Mathews, A., May, J., Grove, M., \& et al. (1991). Assessment of cognitive bias in anxiety and depression using a colour perception task. Cognition and Emotion, 5, 221-238.

Mogg, K., Millar, N., \& Bradley, B. P. (2000). Biases in eye movements to threatening facial expressions in generalized anxiety disorder and depressive disorder. Journal of Abnormal Psychology, 109, 695-704.

Muller, M. M., \& Gruber, T. (2001). Induced gamma-band responses in the human EEG are related to attentional information processing. Visual Cognition, 8, 579-592.

Muller, M. M., Gruber, T., \& Keil, A. (2000). Modulation of induced gamma band activity in the human EEG by attention and visual information processing. International Journal of Psychophysiology, 38, 283-299.

Müller, H. J., \& Rabbitt, P. M. (1989). Reflexive and voluntary orienting of visual attention: time course of activation and resistance to interruption. Journal of Experimental Psychology: Human Perception and Performance, 15, 315-330.

Murphy, F. C., \& Sahakian, B. J. (2001). Neuropsychology of bipolar disorder. British Journal of Psychiatry, 41, s120-127.

Näätänen, R. (1972). Time uncertainty and occurrence uncertainty of the stimulus in a simple reaction time task. Acta Psychologica, 36, 492-503.

Neisser, U. (1963). Decision time without reaction time: Experiments in visual scanning. American Journal of Psychology, 76, 376-385.

Nobre, A. C. (2001). Orienting attention to instants in time. Neuropsychologia, 39, 1317-1328.

Nobre, A. C., Sebestyen, G. N., \& Miniussi, C. (2000). The dynamics of shifting visuospatial attention revealed by event- related potentials. Neuropsychologia, 38, 964-974. 
Persons, J. B., \& Miranda, J. (1992). Cognitive theories of vulnerability to depression: Reconciling negative evidence. Cognitive Therapy and Research, 16, 185-205.

Pfurtscheller, G. (1992). Event-related synchronization (ERS): an electrophysiological correlate of cortical areas at rest. Electroencephalography and Clinical Neurophysiology, 83, 62-69.

Pfurtscheller, G., \& Aranibar, A. (1977). Event-related cortical desynchronization detected by power measurements of scalp EEG. Electroencephalography and Clinical Neurophysiology, 42, 817-826.

Pfurtscheller, G., Stancak, A., \& Neuper, C. (1996). Event-related synchronization (ERS) in the alpha band - an electrophysiological correlate of cortical idling: A review. International Journal of Psychophysiology, 24, 39-46.

Pflugshaupt, T., Mosimann, U. P., Von Wartburg, R., Schmitt, W., Nyffeler, T., \& Müri, R. M. (2005). Hypervigilance-avoidance pattern in spider phobia. Journal of Anxiety Disorders, 19, 105-116.

Posner, M. I. (1978). Chronometric explorations of mind. Hillsdale, N.J.: Lawrence Erlbaum Associates.

Posner, M. I. (1980). Orienting of attention. Quarterly Journal of Experimental Psychology, 32, 3-25.

Posner, M. I., \& Boies, S. J. (1971). Components of attention. Psychological Review, 78, 391-408.

Posner, M. I., \& Cohen, Y. (1984). Components of visual orienting. In H. Bouma (Ed.), Attention and Performance X: control of language processes (pp. 531-556). London: Erlbaum.

Posner, M. I., Nissen, M. J., \& Ogden, W. C. (1978). Attended and unattended processing modes: the role of set for spatial location. In H. L. Pick \& E. Saltzman (Eds.), Modes of perceiving and processing information (pp. 137-157). Hillsdale: Lawrence Erlbaum Associates.

Posner, M. I., \& Petersen, S. E. (1990). The attention system of the human brain. Annual Review of Neuroscience, 13, 25-42.

Posner, M.I., Rafal, R.D., Chaote, L.S., Vaughan, J. (1985). Inhibition of return: Neural basis and function. Cognitive Neuropsychology, 2, 211-228.

Posner, M. I., \& Snyder, C. R. R. (1975). Attention and cognitive control. In R. L. Solso (Ed.), Information processing and cognition, the loyola symposium (pp. 55-85). Hillsdale: Lawrence Erlbaum Associates, publishers.

Posner, M. I., Snyder, C.R.R., Davidson, B.J. (1980). Attention and the detection of signals. Journal of Experimental Psychology, 109, 160-174.

Posner, M. I., Walker, J. A., Friedrich, F. A., \& Rafal, R. D. (1984). Effects of parietal injury on covert orienting of attention. The Journal of Neuroscience, 4, 1863-1874.

Posner, M. I., Walker, J. A., Friedrich, F. A., \& Rafal, R. D. (1987). How do the parietal lobes direct covert attention? Neuropsychologia, 25, 135-145.

Pourtois, G., Grandjean, D., Sander, D., \& Vuilleumier, P. (2004). Electrophysiological correlates of rapid spatial orienting towards fearful faces. Cerebral Cortex, 14, 619633.

Pratt, J., \& Abrams, R.A. (1999). Inhibition of return in discrimination tasks. Journal of Experimental Psychology: Human Perception and Performance, 25, 229-242. 
Rabbitt, P. (1982). Visual search. In C. R. Puff (Ed.), Handbook of research methods in human memory and cognition. New York: Academic Press.

Rafal, R. D., Calabresi, P. A., Brennan, C. W., \& Sciolto, T. K. (1989). Saccade preparation inhibits reorienting to recently attended locations. Journal of Experimental Psychology: Human Perception and Performance, 15, 673-685.

Rafal, R., \& Henik, A. (1994). The neurology of inhibition: Integrating controlled and automatic processes. In D. Dagenbach \& T. H. Carr (Eds.), Inhibitory processes in attention, memory and language (pp. 1-51). San Diego, CA: Academic Press.

Ridderinkhof, K. R. (2002). Micro- and macro-adjustments of task set: activation and suppression in conflict tasks. Psychological Research, 66, 312-323.

Rinck, M., \& Becker, E. S. (2005). A comparison of attentional biases and memory biases in women with social phobia and major depression. Journal of Abnormal Psychology, 114, 62-74.

Rinck, M., \& Becker, E. S. (2006). Spider fearful individuals attentd to threat, then quickly avoid it: Evidence from eye movements. Journal of Abnormal Psychology, 115, 231-238.

Rinck, M., Becker, E. S., Kellermann, J., \& Roth, W. T. (2003). Selective attention in anxiety: Distraction and enhancement in visual search. Depression and Anxiety, 18, $18-28$.

Rizzolatti, G. Riggio, L., Dascola, I., \& Umilta, C. (1987). Reorienting attention across the horizontal and vertical meridians: Evidence in favor of a premotor theory of attention. Neuropsychologia, 25, 31-40.

Robitaille, N., \& Jolicoeur, P. (2006). Fundamental properties of the N2pc as an index of spatial attention: effects of masking. Canadian Journal of Experimental Psychology, 60, 101-111.

Rohner, J. C. (2002). The time-course of visual threat processing: High trait anxious individuals eventually avert their gaze from angry faces. Cognition and Emotion, 16, 837-844.

Rude, S. S., Wenzlaff, R. M., Gibbs, B., Vane, J., \& Whitney, T. (2002). Negative processing biases predict subsequent depressive symptoms. Cognition and Emotion, 16, 423-440.

Ruiter, C. de, \& Brosschot, J. F. (1994). The emotional Stroop interference effect in anxiety: Attentional bias or cognitive avoidance? Behaviour Research and Therapy, 32, 315-319.

Salmelin, R., \& Hari, R. (1994). Characterization of spontaneous MEG rhythms in healthy adults. Electroencephalography and Clinical Neurophysiology, 91, 237-248.

Schmukle, S. C. (2005). Unreliability of the dot probe task. European Journal of Personality, 19, 595-605.

Schupp, H. T., Ohman, A., Junghofer, M., Weike, A. I., Stockburger, J., \& Hamm, A. O. (2004). The facilitated processing of threatening faces: an ERP analysis. Emotion, 4, $189-200$

Semlitsch, H.V., Anderer, P., Schuster, P., \& Presslich, O. (1986). A solution for reliable and valid reduction of ocular artifacts applied to the P300 ERP. Psychophysiology, 23, 695-703.

Shaw, M. L. (1984). Division of attention among spatial locations: A fundamental difference between detection of letters and detection of luminance increments. In $\mathrm{H}$. 
Bouma \& D. G. Bouwhuis (Eds.), Attention and Performance X: control of language processes (pp. 109-121). Hillsdale, NJ: Erlbaum.

Sheer, D. E. (1976). Focused arousal and $40 \mathrm{~Hz}$ EEG. In R. M. Knights \& D. J. Bakker (Eds.), The neuropsychology of learning disorders (pp. 71-87). Baltimore: University Park Press.

Shibata, T., Shimoyama, I., Ito, T., Abla, D., Iwasa, H., Koseki, K., et al. (1999). Attention changes the peak latency of the visual gamma-band oscillation of the EEG. Neuroreport, 10, 1167-1170.

Slagter, H. A., Kok, A., Mol, N., \& Kenemans, J. L. (2005). Spatio-temporal dynamics of top-down control: directing attention to location and/or color as revealed by ERPs and source modeling. Cognitive Brain Research, 22, 333-348.

Slagter, H.A., Kok, A., Mol, N., Talsma, D., \& Kenemans, J.L. (2005). Generating spatial and non-spatial attentional control: An ERP study. Psychophysiology, 42, 428-439.

Snodgrass, J. G. (1969). Foreperiod effects in simple reaction time: Anticipation or expectancy? Journal of Experimental Psychology, 79, 1-19.

Soetens, E. (1990). Sequential effects in two-choice reaction time.Unpublished doctoral dissertation, University of Leiden, Leiden, The Netherlands.

Soetens, E. (1998). Localizing sequential effects in serial choice reaction time with the information reduction procedure. Journal of Experimental Psychology: Human Perception and Performance, 24, 547-568.

Sokolov, A., Lutzenberger, W., Pavlova, M., Preissl, H., Braun, C., \& Birbaumer, N. (1999). Gamma-band MEG activity to coherent motion depends on task-driven attention. Neuroreport, 10, 1997-2000.

Sperling, G. (1984). A unified theory of attention and signal detection. In R. Parasuraman \& D. R. Davies (Eds.), Varieties of attention (pp. 103-181). Orlanda, FL: Academic Press.

Spinhoven, P., Ormel, J., Sloekers, P. P., Kempen, G. I., Speckens, A. E., \& van Hemert, A. M. (1997). A validation study of the Hospital Anxiety Depression Scale (HADS) in different groups of Dutch subjects. Psychological Medicine, 27, 363-370.

Stelmach, L. B., Campsall, J. M., \& Herdman, C. M. (1997). Attentional and ocular movements. Journal of Experimental Psychology: Human Perception and Performance., 23, 823-844.

Stroop, J.R. (1935). Studies of interference in serial-verbal reaction. Journal of EXperimental Psychologyy, 18, 643-662.

Stormark, K. M., Nordby, H., \& Hugdahl, K. (1995). Attentional shifts to emotionally charged cues: Behavioural and ERP data. Cognition and Emotion., 9, 507-523.

Stuirmer, B., Leuthold, H., Soetens, E., Schröter, H., \& Sommer, W. (2002). Control over location-based response activation in the Simon task: behavioral and electrophysiological evidence. Journal of Experimental Psychology: Human Perception and Performance, 28, 1345-1363.

Suslow, T., Dannlowski, U., Lalee-Mentzel, J., Donges, U., Arolt, V., \& Kersting, A. (2004). Spatial processing of facial emotion in patients with unipolar depression: a longitudinal study. Journal of Affective Disorders, 83, 59-63.

Suslow, T., Junghanns, K., \& Arolt, V. (2001). Detection of facial expressions of emotions in depression. Perceptual and Motor Skills, 92, 857-868. 
Sutton, L. J., Teasdale, J. D., \& Broadbent, D. E. (1988). Negative self-schema: The effects of induced depressed mood. British Journal of Clinical Psychology, 27, 188-190.

Talsma, D., Slagter, H. A., Nieuwenhuis, S., Hage, J., \& Kok, A. (2005). The orienting of visuospatial attention: An event-related brain potential study. Cognitive Brain Research, 25, 117-129.

Taylor, S. E., \& Brown, J. D. (1988). Illusion and well-being: A social-psychological perspective on mental health. Psychological Bulletin, 103, 193-210.

Taylor, T.L., \& Klein, R.M. (1998). On the causes and effects of inhibition of return. Psychonomic Bulletin \& Review, 5, 625-643.

Taylor, T.L., \& Klein, R.M. (2000). Visual and motor effects in inhibition of return. Journal of Experimental Psychology, 26, 1639-1656.

Tipper, S.P., Grison, S., \& Kessler, K. (2003). Long-term inhibition of return of attention. Psychological Science, 14, 19-25.

Tipples, J. (2002). Eye gaze is not unique: Automatic orienting in response to uninformative arrows. Psychonomic Bulletin and Review, 9, 314-318.

Tu, J.V. (1996). Advantages and disadvantages of using artificial neural networks versus logistic regression for predicting medical outcomes. Journal of Clinical Epidemiology, 49, 1225-1231.

Tversky, A., \& Edwards, W. (1966). Information versus reward in binary choices. Journal of Experimental Psychology, 71, 680-683.

Van Boxtel, G.J.M., \& Böcker K.B.E. (2004). Cortical measures of anticipation. Journal of Psychophysiology, 18, 61-76.

Van der Lubbe, R. H., Wauschkuhn, B., Wascher, E., Niehoff, T., Kompf, D., \& Verleger, R. (2000). Lateralized EEG components with direction information for the preparation of saccades versus finger movements. Experimental Brain Research, 132, 163-178.

Van der Ploeg, H. M., Defares, P. B., \& Spielberger, C. D. (1980). Handleiding bij de Zelf-Beoordelings Vragenlijst: Een Nederlandstalige bewerking van de Spielberger State-Trait Anxiety Inventory [A manual for the Self-Assessment Questionnaire: A Dutch version ofthe Spielberger State-Trait Anxiety Inventory]. Swets\&Zeitlinger b.v., Lisse.

Van Velzen, J., \& Eimer, M. (2003). Early posterior ERP components do not reflect the control of attentional shifts toward expected peripheral events. Psychophysiology, $40,827-831$.

Van Velzen, J., Forster, B., \& Eimer, M. (2002). Temporal dynamics of lateralized ERP components elicited during endogenous attentional shifts to relevant tactile events. Psychophysiology, 39, 874-878.

Verleger, R., Vollmer, C., Wauschkuhn, B., van der Lubbe, R. H., \& Wascher, E. (2000). Dimensional overlap between arrows as cueing stimuli and responses? Evidence from contra-ipsilateral differences in EEG potentials. Cognitive Brain Research, 10, 99-109.

Wald, F. D. M., Mellenbergh, G. J. (1990). De verkorte versie van de Nederlandse vertaling van de Profile of Mood State (POMS). [The Dutch shortened version of the Profile of Mood State (POMS).]. Nederlands Tijdschrift vande Psychologie en haar Grensgebieden, 45, 86-91.

Walter, W. G., Cooper, R., Aldridge, V. J., McCallum, W. C., \& Winter, A. L. (1964). Contingent negative variation: an electric sign of sensorimotor association and expectancy in the human brain. Nature, 203, 380-384. 
Ward, L. M. (2003). Synchronous neural oscillations and cognitive processes. Trends in Cognitive Sciences, 7, 553-559.

Wijers, A. A., Lange, J. J., Mulder, G., \& Mulder, L. J. (1997). An ERP study of visual spatial attention and letter target detection for isoluminant and nonisoluminant stimuli. Psychophysiology, 34, 553-565.

Williams, J. M. G., Mathews, A., \& MacLeod, C. (1996). The emotional Stroop task and psychopathology. Psychological Bulletin, 120, 3-24.

Williams, J. M. G., Watts, F. N., MacLeod, C., \& Mathews, A. (1988). Cognitive psychology and emotional disorders (1st ed). Chichester, England: Wiley.

Williams, J. M. G., Watts, F. N., MacLeod, C., \& Mathews, A. (1997). Cognitive psychology and emotional disorders (2nd ed). Chichester, England: Wiley.

Woodman, G. F., \& Luck, S. J. (1999). Electrophysiological measurement of rapid shifts of attention during visual search. Nature, 400, 867-869.

Woodman, G. F., \& Luck, S. J. (2003). Serial deployment of attention during visual search. Journal of Experimental Psychology: Human Perception and Performance, 29, 121-138.

Woodrow, H. The measurement of attention. Psychol. Monogr., 1914, 17: no5.

Worden, M. S., Foxe, J. J., Wang, N., \& Simpson, G. V. (2000). Anticipatory biasing of visuospatial attention indexed by retinotopically specific alpha-band electroencephalography increases over occipital cortex. Journal of Neuroscience, 20, 1-6.

Yamaguchi, S., Tsuchiya, H., \& Kobayashi, S. (1994). Electroencephalographic activity associated with shifts of visuospatial attention. Brain, 117, 553-562.

Young, R. C., Biggs, J. T., Ziegler, V. E., \& Meyer, D. A. (1978). A rating scale for mania: Reliability, validity and sensitivity. British Journal of Psychiatry, 133, 429-435. 
$\mathrm{R}$ references 


\section{Dankwoord}




\section{MET DANK AAN.....}

Fren, voor alle begeleiding en de prettige samenwerking! Ik ken niemand met meer parate kennis op zoveel verschillende gebieden; waar ik ook mee aankwam je had altijd een verhaal, suggestie, of tenminste een referentie. Die kennis, je enthousiasme en kritische blik maakten het erg leerzaam om met je samen te werken. Bedankt voor de fijne tijd!

Harald, voor de mogelijkheid om te promoveren op dit mooie project en me zo te kunnen ontwikkelen als onderzoeker.

Anita, voor alle "extra" hulp en je positieve, relativerende kijk op de zaken.

Gerard van Breukelen, voor al je hulp op het gebied van statistiek. De informatiedichtheid van je emails was bijzonder, zeker gezien de snelheid waarmee je altijd antwoordde. Je hulp tijdens dit project was dan ook van grote waarde!

Mijn paranimfen, Anne \& Martien: wat geweldig dat jullie aan mijn zijde willen staan op deze dag. Heel erg bedankt!

Mijn twee kamergenoten: het mag als een cliché klinken, maar ik had me geen betere kamergenoten kunnen wensen. Ik ga jullie dan ook zeker missen!

Lieve Martien, bedankt voor alle inhoudelijke discussies, je humor, gezelligheid, vriendschap en steun. Het was geweldig dat je mijn kamergenoot was. Daarnaast wil ik je bedanken voor het nalezen van en je commentaar op de Nederlandse samenvatting en enkele andere hoofdstukken. Fantastisch hoe je altijd bereid was stukken na te lezen! Lieve Ramona, bedankt dat je zo'n fantastische, lieve en positieve kamergenote was die zorgde voor de nodige gezelligheid en steun. Ook je bureau zal ik nooit vergeten :) . Bedankt ook voor het nalezen van Hoofdstuk 5 en al je Engels-tips.

Anne, voor je aanhoudende vertrouwen in me, je hulp en natuurlijk alle gezelligheid! Bedankt ook voor je commentaar op Hoofdstuk 5

Marieke $W$., voor alle rondjes-rond-het-gebouw. Ooit door ons in het leven geroepen en nog steeds de beste manier om te pauzeren! Daarnaast bedankt voor alle gezelligheid en je steun!

Judith Peters, voor het delen van alle leuke maar ook moeilijke zaken rond ons AIObestaan en een opbeurende yell op zijn tijd.

Saskia Ranson, Joep van der Heiden, Charlotte Sinke, Olga Reneerkens, voor jullie hulp en inzet bij de dataverzameling en in het bijzonder Saskia voor alle nuttige brainstorms.

Het secretariaat: Truus, Jessie, Nicole en daarnaast Liane, voor alle hulp en in het bijzonder Jessie voor al het geregel aan het eind van het project.

De technische dienst: Ron, Johan en Jacco, omdat EEG onderzoek zonder jullie niet mogelijk is!

Heleen Slagter, voor al je hulp en nuttige discussies via de mail vanuit Amsterdam en later vanuit de VS.

Durk Talsma en Tineke Grent-'t Jong, voor jullie hulp en antwoorden via de mail op al mijn aandacht-en-ERP-vragen.

José van Velzen \& Martin Eimer, for the opportunity to visit the Brain \& Behaviour Lab and all useful discussions in such a short time. José bedankt voor je gastvrijheid. Het waren een aantal hele fijne en nuttige dagen! Daarnaast bedankt voor de discus- 
sies die we via de mail voerden. And thank you Martin, for your helpful comments on Chapter 2.

Sander Los, voor je commentaar op Hoofdstuk 4.

Nienke Jabben, Daniëlle Ummels, Baer Arts, Lydia Krabbendam en Jim van Os, voor de mogelijkheid de klinische studie op te zetten binnen jullie project en in het bijzonder bedankt Nienke en Daniëlle voor al jullie hulp bij het werven van deelnemers.

Richel Lousberg, omdat de deur altijd open stond!

Alle deelnemers aan het onderzoek: zonder wie al dit onderzoek niet mogelijk was geweest.

Alle collega's van $E P$, voor de fijne sfeer op de afdeling.

Jaargenoten AIOs EPOS, voor de gezelligheid tijdens de EPOS-dagen en cursussen.

Manon Grond, voor je vriendschap, ondanks de schaarste van ons contact en voor Indesign-in-Vogelvlucht en de Hoofdstuk-teller!

Hub Elmers, voor de prachtige kaft!

Karin, Esther, Ivo "Bolle" \& alle andere TBCtjes: jullie vormden de perfecte afleiding en relativering van het AIO-bestaan en waren op die manier ook essentieel voor de totstandkoming van mijn proefschrift. Bedankt voor jullie vriendschap.

Pap, Mam, Linda, Michael, Sjaak, Annemie, Marieke \& Roel, voor jullie vertrouwen in mij, jullie steun, interesse en alle gezelligheid. En voor alle muzikale ontspanning in de vorm van nieuwe muziek in het bijzonder pap en Michael!

Caspar: lieve Caspar, wanneer ik terugkijk op de afgelopen jaren is het moeilijk in een paar zinnen te beschrijven waar ik je allemaal voor wil bedanken. Laat ik dit daarom samenvatten in "alles", maar in het bijzonder wil ik je bedanken dat je er altijd voor me was! 
D dankwoord 


\section{Curriculum Vitae}




\section{CURRICULUM VITAE}

Ellen Jongen was born in Heerlen (the Netherlands) on April 18th 1979. She attended the Sintermeerten College in Heerlen and obtained her VWO diploma in 1997. In 1997 she started studying Psychology at Maastricht University, where she also worked as a teaching assistant and research assistant. In 2001 she graduated and received her master's degree in Biological Psychology (cum laude). Her master's thesis described an ERP study on pain perception which she performed at the department of Clinical and Experimental Psychiatry of the Academic Hospital in Maastricht. In December 2001 she began her PhD-project at the department of Experimental Psychology at Maastricht University. Since February 2007 she is a postdoctoral fellow at the Department of Developmental Biopsychology at Maastricht University.

\section{Publications}

Jongen, E.M.M., Smulders, F.T.Y., \& Van Breukelen, G.J.P. (2006). Varieties of attention in neutral trials: Linking RT to ERPs and EEG frequencies. Psychophysiology, 43, 113-125.

Jongen, E.M.M. \& Smulders, F.T.Y. (in press). Sequence effects in a spatial cueing task: Endogenous orienting is sensitive to orienting in the preceding trial. Psychological Research / Psychologische Forschung.

Jongen, E.M.M., Smulders, F.T.Y., Ranson, S.M.G., Arts, B.M.G., \& Krabbendam, L. (in press). Attentional bias and general orienting processes in bipolar disorder. Journal of Behavior Therapy and Experimental Psychiatry.

Giesbrecht, T., Jongen, E.M.M., Smulders, F.T.Y., \& Merckelbach, H. (2006). Dissociation, resting EEG, and subjective sleep experiences in undergraduates. Journal of Nervous and Mental Disease, 194, 362-368.

Lousberg, R., Vuurman, E., Lamers, T., van Breukelen, G., Jongen, E., Rijnen, H., Maessen, C., \& Hermens, H. (2005). Pain report and pain-related evoked potentials operantly conditioned. Clinical Journal of Pain, 21, 262-271.

\section{Manuscripts}

Jongen, E.M.M., Van der Heiden, J.S.H., \& Smulders, F.T.Y. (2006). Lateralized ERP components related to attentional orienting in an all-go and a go-nogo task: Discriminating shifts of attention from sensory aspects of the cue. Manuscript in revision.

Jongen, E.M.M. \& Smulders, F.T.Y. The time course of general orienting and attentional bias for emotional stimuli in induced and naturally-occurring dysphoria: response times and eye movements. Manuscript in preparation.

\section{Conference presentations}

Smulders, F.T.Y., Jongen, E., Meijer, E. (2003). Perceptual load modulates ERP manifestations of endogenous attentional shifts. Poster presented at "Congress at the Feder- 
ation of European Psychophysiology Societies" in Bordeaux, France. (abstract published in Journal of Psychophysiology, 2003, 17, S64).

Jongen, E.M.M., Smulders, F.T.Y., \& Van Breukelen, G.J.P. (2004). Electrophysiological measurement of shifts of attention following a neutral, noninformative cue. Poster presented at the summer conference of the "Nederlandse Vereniging of Psychonomie" in Maastricht, the Netherlands.

Jongen, E.M.M., Smulders, F.T.Y., \& Van Breukelen, G.J.P. (2004). Electrophysiological measurement of attention following neutral, noninformative cues. Paper presented at the Dutch "EndoNeuroPsycho Meeting" in Doorwerth, the Netherlands.

Jongen, E.M.M., Smulders, F.T.Y., Van Breukelen, G.J.P., \& Merckelbach, H.G.L.J. (2004). Electrophysiological measurement of attention following neutral, noninformative cues. Poster presented at the annual meeting of the "Society for Psychophysiological Research" in Santa Fe, New Mexico, USA. (Abstract published in Psychophysiology, 2004, 41, S70).

Jongen, E.M.M., Smulders, F.T.Y. (2004). Analyses of sequence effects in endogenous spatial cueing: evidence for long-term inhibition of return. Poster presented at "Seminar on the neuroscience of cognitive control" in Ghent, Belgium.

Jongen, E.M.M., Smulders, F.T.Y., \& Van der Heiden, J.S.H. (2005). ERP components related to attentional orienting. Poster presented at "EPOS/NWO Workshop Control in Attention and Action: Neurocognitive Systems and Mechanisms" in Amsterdam, the Netherlands.

Jongen, E.M.M., Smulders, F.T.Y. \& Van der Heiden, J.S.H. (2005). ERP components related to attentional orienting. Poster presented at " Tagung experimentell arbeitender Psychologen (TeaP)" in Regensburg, Germany.

Jongen, E.M.M. \& Smulders F.T.Y. (2005). Electrophysiological measurement of attentional bias in induced and naturally occurring dysphoria. Poster presented at "EPOS EPP KLI Symposium on Implicit Cognition and Attention in Experimentel Psychopathology and health research" in Maastricht, the Netherlands.

Jongen, E.M.M., Smulders, F.T.Y., \& Van der Heiden, J.S.H. (2005). ERP components related to orienting of attention. Poster presented at "IX International Conference on Cognitive Neuroscience (ICON9)" in Havana, Cuba.

Jongen, E.M.M. \& Smulders, F.T.Y. (2005). Electrophysiological measurement of attentional bias in induced and naturally occurring dysphoria. Poster presented at "IX International Conference on Cognitive Neuroscience (ICON9)" in Havana, Cuba.

Jongen, E.M.M. \& Smulders, F.T.Y. (2005). Electrophysiological measurement of attentional bias in induced and naturally occurring dysphoria. Poster presented at "NWO summerschool the Heart and the Mind: approaches to emotion and attention " in Doorwerth, the Netherlands.

Jongen, E.M.M. \& Smulders, F.T.Y. (2006). Lateralized components elicited after cue onset: orienting or physical appearance of the cue? Paper presented during short stay at the Brain and Behaviour lab in London, England.

Jongen, E.M.M., Smulders, F.T.Y., \& Van der Heiden, J.S.H. (2006). ERP components related to attentional orienting. Paper presented at the "Winter conference of the Dutch Society for Psychophysiology" in Utrecht, the Netherlands. 
Jongen, E.M.M., Smulders, F.T.Y., Ranson, S.M.G., Arts, B.M.G., \& Krabbendam, L. (2006). Attentional bias in bipolar disorder. Paper presented at the EPOS symposium "Affective modulation of cognitive performance" in Leiden, the Netherlands.

\section{Awards}

SPR Poster-award: Jongen, E.M.M., Smulders, F.T.Y., Van Breukelen, G.J.P., \& Merckelbach, H.G.L.J. (2004). Electrophysiological measurement of attention following neutral, noninformative cues. Poster presented at the annual meeting of the "Society for Psychophysiological Research" in Santa Fe, New Mexico, USA. (Abstract published in Psychophysiology, 2004, 41, S70) 